BATTLE BETWEEN INSECT EGGS AND HOST PLANTS

Ecology and evolution of pierid egg-induced responses in Brassicaceae

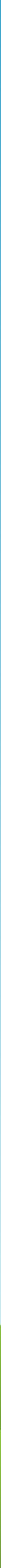




\section{Propositions}

1. Even closely related species can take very different approaches to ensure offspring fitness.

(this thesis)

2. Egg-induced plant responses have been overlooked in breeding for host-plant resistance.

(this thesis)

3. Forced open access of scientific work via shadow libraries will do more harm than good in the long run.

4. Scientific progress has been substantially promoted by the use of English, rather than any other language, as the modern international scientific language.

5. Providing incentives for co-parenting is vital to improve well-being of children and society as a whole.

6. To combat climate change effectively protection and restoration of bogland is more important than reforestation.

Propositions belonging to the thesis, entitled

Battle between insect eggs and host plants - Ecology and evolution of pierid egg-induced responses in Brassicaceae

Eddie Griese

Wageningen, 4 October 2021 


\section{Battle between insect eggs and host plants}

Ecology and evolution of pierid egg-induced responses in Brassicaceae

Eddie Griese 


\section{Thesis committee}

\section{Promotors}

Prof. Dr Marcel Dicke

Professor of Entomology

Wageningen University \& Research

Prof. Dr M. Eric Schranz

Professor of Biosystematics

Wageningen University \& Research

\section{Co-promotors}

Dr Nina E. Fatouros

Associate professor, Biosystematics Group

Wageningen University \& Research

Prof. Dr Monika Hilker

Professor of Applied Zoology / Animal Ecology

Freie Universität Berlin, Germany

\section{Other members}

Prof. Dr Duur K. Aanen, Wageningen University \& Research

Prof. Dr Astrid T. Groot, University of Amsterdam

Dr Bart A. Pannebakker, Wageningen University \& Research

Dr Guusje Bonnema, Wageningen University \& Research

This research was conducted under the auspices of the Graduate School of Experimental Plant Sciences (EPS) 


\title{
Battle between insect eggs and host plants Ecology and evolution of pierid egg-induced responses in Brassicaceae
}

\author{
Eddie Griese
}

Thesis

submitted in fulfillment of the requirements for the degree of doctor at Wageningen University

by the authority of the Rector Magnificus,

Prof. Dr. A.P.J. Mol,

in the presence of the

Thesis Committee appointed by the Academic Board

to be defended in public

on Monday, 4 October 2021

at 1:30 p.m. in the Aula. 


\section{Eddie Griese}

Battle between insect eggs and host plants

Ecology and evolution of pierid egg-induced responses in Brassicaceae 215 pages

$\mathrm{PhD}$ thesis, Wageningen University, Wageningen, The Netherlands (2021) With references, with a summary in English

ISBN 978-94-6395-864-6

DOI: https://doi.org/10.18174/549144 


\section{Contents}

1 General Introduction $\quad 7$

2 Plant response to butterfly eggs: inducibility, severity and success of egg-killing leaf necrosis depends on plant genotype and egg clustering 17

Supplementary Material 42

3 Effects of oviposition by Pieris brassicae and plant accession on plant $\begin{array}{ll}\text { volatiles and behaviour of three insect species } & 47\end{array}$

$\begin{array}{ll}\text { Supplementary Material } & 69\end{array}$

4 Plant responses to butterfly oviposition partly explain preference-performance relationships on different brassicaceous species 81

$\begin{array}{ll}\text { Supplementary Material } & 101\end{array}$

5 Insect egg-killing: A new front on the evolutionary arms race between $\begin{array}{ll}\text { brassicaceous plants and pierid butterflies } & 111\end{array}$

$\begin{array}{ll}\text { Supplementary Material } & 133\end{array}$

6 General Discussion 155

$\begin{array}{llr}7 & \text { Bibliography } & 173\end{array}$

$\begin{array}{ll}\text { Summary } & 201\end{array}$

$\begin{array}{lr}\text { Acknowledgements } & 207\end{array}$

$\begin{array}{ll}\text { About the author } & 211\end{array}$

$\begin{array}{ll}\text { Educational statement } & 213\end{array}$ 
For Kasia 
1

Chapter 1

General Introduction

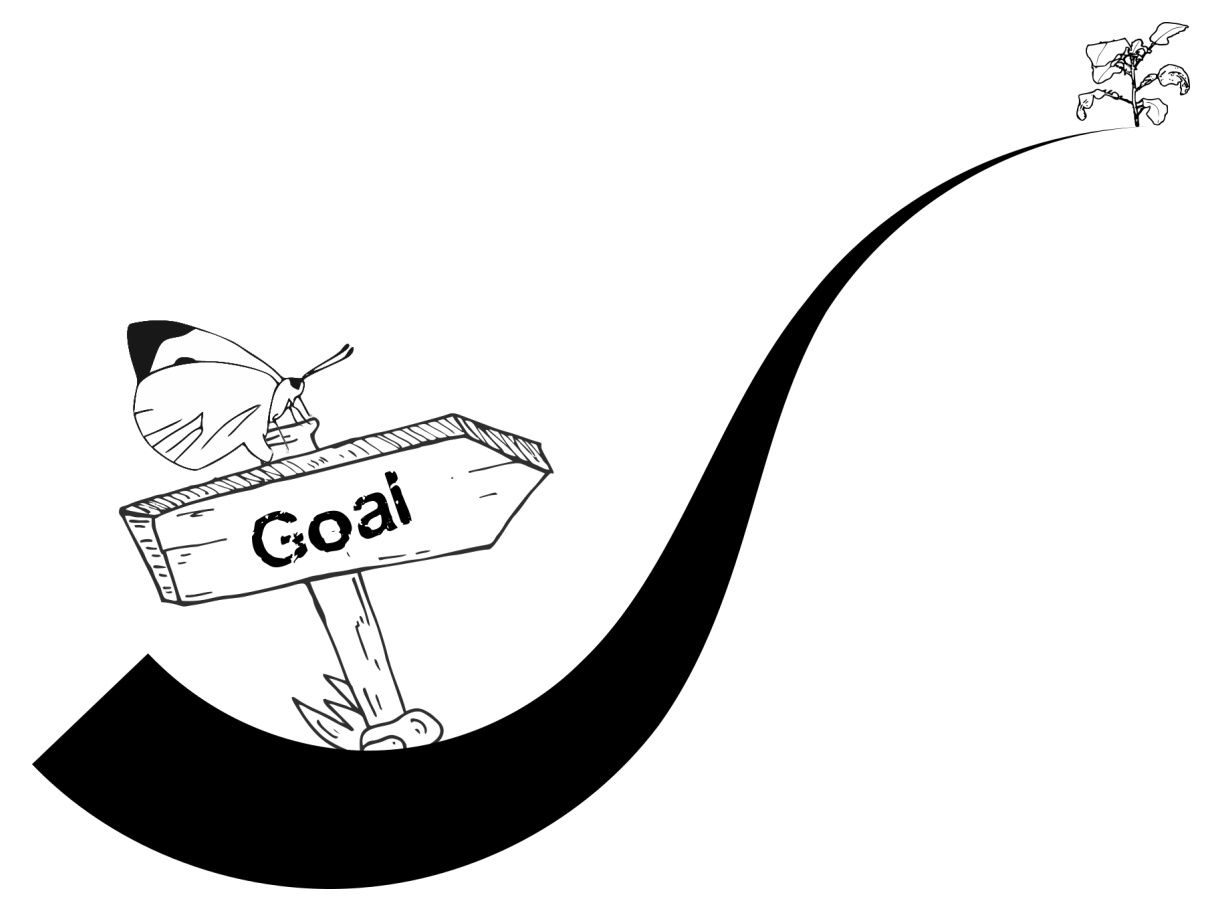

7 


\section{Plant insect interactions}

Since the evolutionary dawn of angiosperms in the Jurassic (between 208 and 145 million years ago) insects have had major biotic interactions with flowering plants and both had major influence on the other's evolutionary history (Grimaldi, 1999). The interactions continue today with nearly all of the 250,000 angiosperm species extensively interacting with insects. Around half of the one million insects using plants as a food source, and the majority of terrestrial biomass is comprised of plants and their herbivore insects. Therefore, investigating the evolution of insect-plant interactions is crucial to understand global patterns of terrestrial biodiversity (Schoonhoven et al., 2005; Farrell and Mitter, 1994). In the course of 300 million years of plant-insect coevolution, plants developed sophisticated defence mechanisms against insect attackers. While mechanical barriers in plants can prevent some insect feeding (Ehrlich and Raven, 1964), chemical defences play an important role as well. Secondary plant metabolites have long been considered a defensive tool against insect herbivores (Fraenkel, 1959; Thorsteinson, 1960). In an inspiring review Ehrlich and Raven (1964) suggested that development of novel chemical defences in some plant species would allow these plants to minimize herbivory and this would then lead to rapid radiation of the plant clade into new species. After a while some phytophagous insects then would evolve the ability to feed on the plant clade with its novel metabolites and quickly radiate over the plant clade to fill available food niches. This escape and radiate scenario was suggested by Ehrlich and Raven (1964) as an explanation for the diversity of plants and insect herbivores on earth.

Two main approaches to study this proposed coevolutionary arms race were used in the following decades. One the one hand in depth studies looking at the interaction between a small number of specific herbivores and plant species can yield information on the nature of adaptations and counteradaptations between these species. For example, Brassicales, especially Brassicaceae evolved the glucosinolate-myrosinase system. Upon damage (mechanical or pest attack) glucosinolates stored in the plant cells come into contact with degrading enzymes (myrosinases) and can be hydrolysed into several toxic substances (mainly isothiocyanates and nitriles). Specialist herbivores such as pierid butterflies evolved counteradaptations to disarm this "mustard oil bomb", allowing them to feed on brassicacaeous plants.

On the other hand, macro-evolutionary studies showing similar phylogenetic branch- 
ing patterns for plant and herbivore species, can reveal the shared evolutionary history of herbivore and plant species (Figure 1.1). The lack of good phylogenetic data from both insects and their host plants was a big issue in showing coevolution directly in the 1960s. Recently, a few robust studies have confirmed that novel chemical defences in Brassicaceae are followed by an increase in speciation rate for these plants and later in time by pierid butterflies (Wheat et al., 2007; Edger et al., 2015). Studies like these help to substantiate the long-held dogma that plant secondary metabolites and counteradaptations against them lead to an escalating arms race between plants and their insect herbivores.

\section{Chemical arms race}

Specific defensive metabolites keep most herbivores at bay. Certain plant families are often associated with particular defensive compounds, which will defend the plants reasonably well against most herbivores. The narrow host range of insect herbivores can, at least in part, be explained by chemical compounds of the plants. Usually, they are restricted to single plant families or even only few closely related plant species (Schoonhoven et al., 2005). Typically, Apiaceae are associated with furanocoumarins and Brassicaceae are associated with the sulphur-based glucosinolate-myrosinase defence system (Berenbaum, 1983; Futuyma and Agrawal, 2009). However, those typical secondary metabolites are not always limited to one specific family. As for example furanocoumarins are found in at least eight plant families (Berenbaum, 1983). Furthermore, plants of a certain family can also evolve chemical defences not based on their typical secondary metabolites. The Brassicaceae for example, evolved several other defensive compounds after having evolved the nitrogen-sulphur-based glucosinolate-myrosinase defence system, such as tropane alkaloids (Brock et al., 2006), cucurbitacins (Sachdev-Gupta et al., 1993), and cardenolides (Makarevich, 1992).

Nevertheless, coevolutionary patterns between herbivores and host plants have been shown. The plant family of the Brassicaceae coevolved with the Lepidoptera family Pieridae, the whites and sulphurs. Especially the butterfly subfamily Pierinae are closely associated with Brassicaceae hosts. It was demonstrated that the evolution of nitrilespecifier proteins (NSPs) enabled the radiation of the Pierinae (Wheat et al., 2007). Furthermore, Edger et al. (2015) showed that a speciation rate shift can be observed in 
the Brassicales when new glucosinolates are added to their chemical defence. Similarly, the Pieridae butterflies diversified in the same time frame (Edger et al., 2015).

Novel defences can give plants the edge for a while in the escalating arms race, e.g. the development of cardenolides in Erysimum (Züst et al., 2020). However, coevolution between insects and their host plants is not only driven by chemical defence. As proposed by Singer and Stireman (2005) tritrophic interactions between plants, herbivores and predators or parasitoids might have a big impact on shaping the evolution of the involved species. Both, the traditional bitrophic and more modern tritrophic ideas of coevolution typically focus on the feeding life stages of herbivores. In this thesis, I introduce another life stage as a potential candidate for playing a role in coevolution between plant species and their insect herbivores: the insect egg.

\section{Defences against eggs}

Defending against eggs is an important part of plant defence. Since every egg killed by the plant before hatching is one less larva feeding on it, defences against eggs have an enormous potential. Due to this potential, egg-killing defences are widespread among plants. Gymnosperms (pine trees) as well as several taxa within the Angiosperms are known to perceive and respond to eggs, lowering egg survival (Fatouros et al., 2016). In general, plant defences can either be constitutively expressed or induced after contact with the herbivore. Constitutively expressed leaf structures such as trichomes have been described to affect the survival of deposited eggs. Higher trichome density can either be beneficial (Benedict et al., 1983) or detrimental for eggs (Schillinger and Gallun, 1968). Furthermore, trichome density already affects the oviposition choice of gravid females (Schillinger and Gallun, 1968; Benedict et al., 1983; Silim Nahdy et al., 1999), which could be interpreted as a defence against eggs. However, more prominently studied are defences against insect eggs induced after oviposition. A wide variety of plant species use direct defences against eggs. Ovicidal substances, neoplasm formation, egg crushing via wound-tissue growth and leaf necrosis are described as lowering egg survival (Hilker and Fatouros, 2015). Furthermore, oviposition can induce the release of specialized volatile compounds, which in turn attract egg- or larval parasitoids to the oviposition site (Hilker and Fatouros, 2015; Fatouros et al., 2012; Pashalidou et al., 2015a). This tritrophic interaction can be understood as an indirect resistance against 
insect eggs. Lastly, eggs can be used to prime defences against hatching larvae, making anti-larvae responses swifter and harsher compared to non-induced plants (Hilker and Fatouros (2015) and references therein). The direct and indirect egg-killing responses as well as the priming of plant defences can have a substantial impact on herbivore fitness. This means counteradaptations to egg defences can be favoured by natural selection. Therefore, an evolutionary arms race involving herbivores and plant responses to eggs is likely to occur. While some counteradaptations to egg defences have been studied, there is a lack of studies demonstrating an arms race involving egg defences.
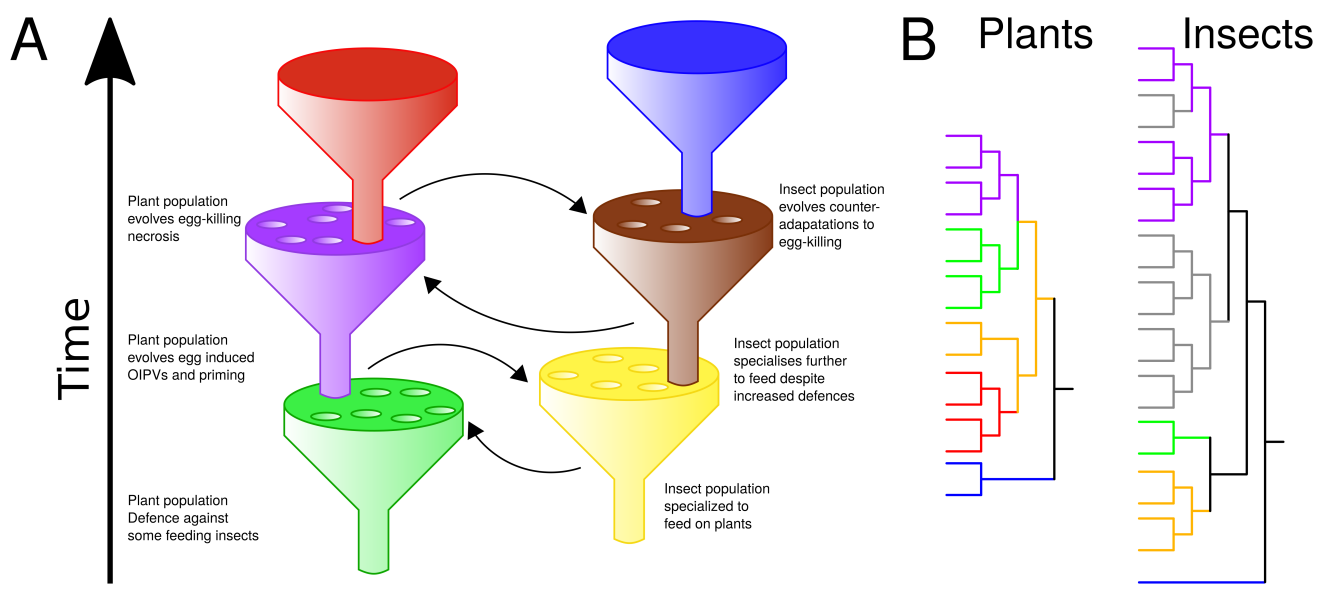

Figure 1.1: Successive counteradaptations between species locked in an arms race scenario can lead to phylograms in which specific adaptations are found in particular monophyletic species groups. A) In an evolutionary arms race scenario both populations involved exert selection pressure on the other after the evolution of new counteradaptations (arrows). Some parts of either population will evolve a new way to combat the other side, escalating into an arms race where plant defences and insect counteradaptations become more complex as time moves along. The single steps presented are hypothetical and could also have happened in a different order in time. B) Example scheme of two evolutionary linked phylogenies, in this case the host plants and herbivorous insects. Plants would evolve a specific new or improved defence, and some insects within the clade developed the ability to overcome these defences (same colour for coevolved plant and insect clades). However, some insects in the phylogeny switched to a different host plant at some point in time (grey species) and some plant defences were not yet overcome by the insects (light green plant species).

\section{Study system}

In order to improve our understanding of plants' egg-killing defences and how these might shape the interactions with herbivores and their eggs, I used the Brassicaceae plant family and their pierid herbivores as model organisms. The diverse Brassicaceae, which 
occur all around the world, contain important crop plants such as cabbage (Brassica oleracea), rapeseed (B. napus) and mustard (Sinapis alba). Furthermore, the model plant Arabidopsis thaliana is also a member of the family. These facts lead to a wealth of data being gathered on Brassicaceae plants. Additionally, in recent years the phylogeny and relationships in the Brassicaceae has become more resolved and understood (Al-Shehbaz, 2012; Guo et al., 2017; Huang et al., 2015). The typical Brassicales glucosinolate-myrosinase resistance system has been diversified in the evolutionary history of the Brassicaceae (Edger et al., 2015) and can keep most non-specialist insects from feeding on them. Yet, specialist herbivores such as diamond-back moths (Plutella xylostella) and pierid butterflies developed detoxification mechanisms to overcome this defence system (Heidel-Fischer and Vogel, 2015; Wheat et al., 2007). Recently, a coevolutionary relationship between the Brassicales and the butterfly family Pieridae has been shown to exist (Edger et al., 2015). The diversification of glucosinolates can explain some of the speciation rate shifts observed. As NSPs are used by pierid caterpillars to detoxify the glucosinolates, specificity exists among the pierid butterflies to feed on and detoxify specific brassicaceous plant species containing specific glucosinolates.

Furthermore, several brassicaceous plant species have been demonstrated to respond to insect eggs (Fatouros et al., 2016), by induction of OIPVs, to attract egg parasitoids, changes of leaf surfaces to arrest egg parasitoids, and the expression of hypersensitive response (HR)-like necrosis (Fatouros et al., 2005; Fatouros et al., 2014; Shapiro and DeVay, 1987; Conti et al., 2010; Hilker and Fatouros, 2015). Most of those egg-induced responses have been demonstrated to lower egg survival of specialist pierid butterflies (Shapiro and DeVay, 1987; Fatouros et al., 2014). Generalist moths like the cabbage moth (Mamestra brassicae) do not elicit the same egg-induced responses in B. nigra (Fatouros et al., 2012; Pashalidou et al., 2013). This suggests the responses are specific for some herbivore eggs and not a general one to insect eggs (as shown for A. thaliana plants (Bruessow et al., 2010)). Whether egg-induced defence responses are subject to evolutionary arms races has not yet been researched. For an example of an expected egg-induced resistance arms race see Figure 1.1A.

In my thesis, I started off from a small study system containing only $B$. nigra and $P$. brassicae (chapter 2). I studied the role of HR-like symptoms, which plants show in response to egg deposition, both under greenhouse and natural conditions. Thereafter, I expanded the number of organisms included in the study system. In chapter 3, I studied 
the tritrophic interactions and syngerstic effects of direct (HR-like) and indirect egginduced responses, using egg- and larval parasitoids, and tried to resolve the question whether these responses are beneficial for B. nigra. In chapter 4, I increased my setup to seven brassicaceous species, and an additional butterfly species, the solitary $P$. rapae. Here, I studied, in addition to HR-like necrosis, the plant-mediated effects of egg deposition on the preferences and performances of the different butterfly species. Finally, in chapter 5, a broad family-wide approach of the Brassicaceae and Pieridae was chosen to unravel patterns of expression (Brassicaceae side) and elicitation (Pieridae side) of egg-killing HR-like necrosis.

\section{Research objectives}

In this thesis, brassicaceous plants are used to shed light on the variability of resistance against insect eggs and how egg-induced responses change insect behaviour towards those plants. Furthermore, I want to investigate how a specific egg-resistance trait against specialist butterflies might have evolved within the Brassicaceae plant family. The main objective of the research was to show how egg deposition induces changes within different plants, which in turn influence the eggs themselves, shapes the interaction with the herbivores and natural enemies and finally how resistance against specialist herbivore eggs might have evolved within the whole plant family.

\section{Thesis outline}

This thesis shows how egg deposition leads to egg killing and changes in insect behaviour (including preference) and how those egg-induced responses might have evolved in the family of the Brassicaceae. For an overview over the study system see Figure 1.2.

In chapter 2 greenhouse and semi-field studies were used to study the effect of a direct egg-killing trait in B. nigra induced by eggs of a specialist lepidopteran, Pieris brassicae. The in-depth look at a small-scale study system allows a close look into the effectiveness of HR-like necrosis as an egg-killing trait. I specifically assessed whether or not the leaf necrosis would lead to egg killing against $P$. brassicae eggs under greenhouse and field conditions. To assess this, I developed a scoring system for the strength of HR-like necrosis and compared egg survival on plants expressing (HR+) and not ex- 


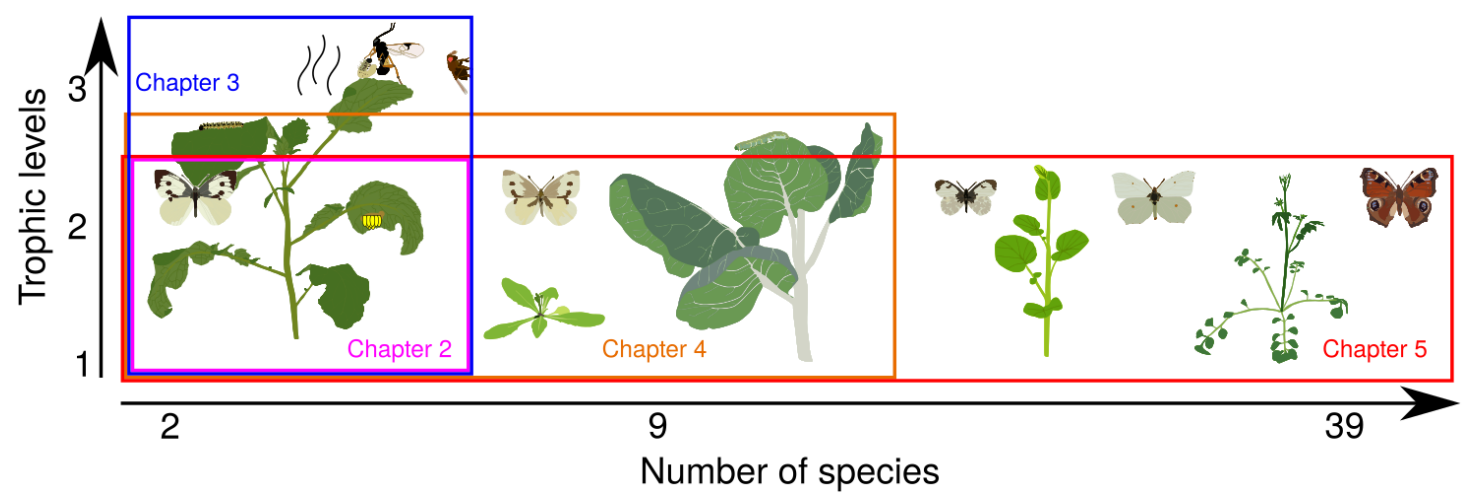

Figure 1.2: Scheme of the chapters in this thesis. It highlights the trophic levels investigated in each chapter as well as species (plants and butterflies) used. The study system expands from the second chapter, where only $B$. nigra plants and P. brassicae were used, over chapters 3 and 4 to a family-wide approach in chapter 5, where a selection of Brassicaceae plants and Pieridae butterflies were tested.

pressing (HR-) HR-like necrosis after egg deposition. Furthermore, I wanted to study whether or not variation in the occurrence of egg-induced HR-like necrosis between different $B$. nigra accessions existed. In order to do so, I grew four different $B$. nigra accessions and compared their expression of HR-like necrosis after P. brassicae egg deposition.

Building on the results of chapter 2, in chapter 3 I studied whether or not different $B$. nigra accessions would vary with respect to their egg-induced blends of plant volatiles and the behavioural responses of the herbivores, an egg- and a larval parasitoid. Furthermore, I tried to explain the behavioural responses by investigating the plants' volatile emission induced by oviposition. Finally, I tried to link the behavioural responses to plant fitness. In other words, I tried to evaluate whether or not plant responses to insect eggs (HR-like necrosis, OIPVs and priming) are an adaptive resistance response. I studied these interactions again under laboratory and field conditions to observe preferences of the herbivore $P$. brassicae, its egg parasitoid Trichogramma evanescens, and the larval parasitoid Cotesia glomerata.

For chapter 4, I expanded the study system and used two butterfly species, $P$. brassicae and $P$. rapae, as well as eight brassicaceous plant species to investigate whether egglaying preferences and offspring performance of the butterfly species differed with regards to those eight plant species. Special attention was given to the effect of egginduced defences (HR-like necrosis and egg-mediated priming) in the outcome of these 
preference-performance relationships. In order to do so, butterflies could choose between all plant species for egg deposition. Butterflies were then manipulated to lay eggs on all plant species. After hatching, caterpillars were either allowed to feed on previously egg-free or egg-laden plants of the same species their eggs were laid onto. Egg mortality was recorded, and larval performance measured by weighing individual caterpillars three and seven days after hatching.

In chapter 5 I use a macroevolutionary approach to look at the expression of HR-like necrosis as an egg killing trait within the Brassicaceae family. First by phenotyping 28 Brassicaceae and 3 Cleomaceae plant species I investigated the prevalence of HR-like necrosis within the plant family and looked into the potential for a phylogenetic signal between HR-like necrosis and specific plant species. Then I evaluated the effect of HRlike necrosis on egg survival of specialist Pieris butterflies. Finally, I tested eggs/egg washes of eight different butterfly species to screen for elicitation of HR-like necrosis in B. nigra and a potential link of the elicitation in the subfamilies of the Pieridae.

Finally, in chapter 6 the general discussion of my thesis I placed my thesis into a broader context by reviewing the literature available. I further speculate on implications the results presented in this thesis could have on related fields of research. Lastly, I discuss future research potentially resulting from my thesis. 
Chapter 2

Plant response to butterfly eggs: inducibility, severity and success of egg-killing leaf necrosis depends on plant genotype and egg clustering

Eddie Griese, Marcel Dicke, Monika Hilker \& Nina E. Fatouros

Published in: Scientific Reports (2017) 7 : 7316

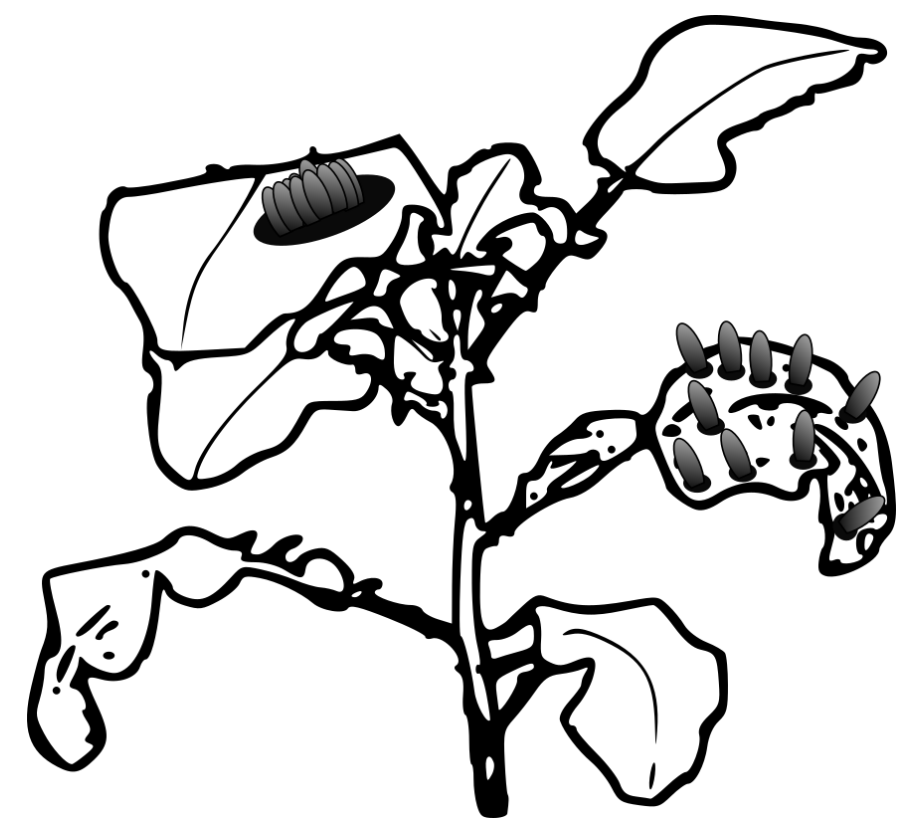




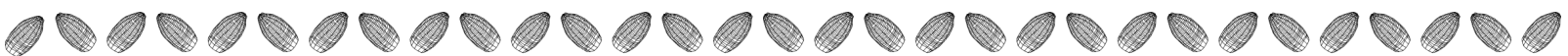 \\ Abstract \\ Plants employ various defences killing the insect attacker in an early stage. Oviposition by cabbage white butterflies (Pieris spp.) on brassicaceous plants, including Brassica nigra, induces a hypersensitive response (HR)-like leaf necrosis promoting desiccation of eggs. To gain a deeper insight into the arms race between butterflies and plants, we conducted field and greenhouse experiments using different $B$. nigra genotypes. We in- vestigated variation in HR and consequent survival of $P$. brassicae egg clusters. Impact of egg density, distribution type and humidity on HR formation and egg survival was tested. HR differed among plant genotypes as well as plant individuals. Egg density per plant did not affect HR formation. Remarkably, egg survival did not depend on the formation of HR, unless butterflies were forced to lay single eggs. Larval hatching suc- cess from single eggs was lower on plants expressing HR. This may be due to increased vulnerability of single eggs to low humidity conditions at necrotic leaf sites. We con- clude that effectiveness of HR-like necrosis in B. nigra varies with plant genotype, plant individual and the type of egg laying behaviour (singly or clustered). By clustering eggs, cabbage white butterflies can escape the egg-killing, direct plant defence trait.}

\section{Introduction}

Plant defences against herbivory largely exhibit phenotypic plasticity, which is a powerful means to cope with threats because a target with variable traits is less likely to be hit (Adler and Karban, 1994). Plants are well known to defend themselves against herbivorous insects by a wide range of strategies, some of which are genotypically fixed and employed constitutively, and numerous others are based on phenotypic changes that are induced in response to herbivore attack (Agrawal and Karban, 2000; Agrawal, 2001; Lucas-Barbosa et al., 2013; Stam et al., 2014). The range of phenotypic changes of a plant is determined by its genotype and its environment (Des Marais et al., 2013). Hence, different accessions of a plant species exposed to variable environmental conditions may display variability with respect to the inducibility of their defensive responses.

Egg deposition by insects onto plants often represents the first step of infestation. 
Plant defences induced by insect oviposition may target the eggs themselves or the herbivorous larvae. Oviposition can inform plants about impending herbivory, and the "warned" plants prepare their defences against hatching larvae. Various plant responses are known to directly kill the eggs by ovicidal compounds, growth of plant tissue around the eggs, or the formation of neoplasms or necrotic leaf tissue (reviewed by refs (Hilker and Fatouros, 2016; Hilker and Fatouros, 2015; Fatouros et al., 2016)). The formation of necrotic leaf tissue in response to insect egg deposition leads to detachment of eggs from leaves or desiccation of eggs and has been described as a hypersensitive-like response (HR) (Shapiro and DeVay, 1987; Balbyshev and Lorenzen, 1997; PetzoldMaxwell et al., 2011; Reymond, 2013; Fatouros et al., 2014; Pashalidou et al., 2015a; Gouhier-Darimont et al., 2013).

From the insect's perspective, the oviposition behaviour of a mother is an important determinant of successful reproduction (Courtney, 1984; Ramos et al., 2012; Fortuna et al., 2013). Females invest in survival of their progeny by e.g. hiding the eggs, protecting them with sticky secretions that prevent parasitism, endowing them with predatordeterring compounds or by increasing egg size (Hilker and Fatouros, 2015; Desurmont and Weston, 2011; Blum and Hilker, 2002). In herbivorous insects, clustering of eggs has been suggested as an adaptation to plant defences (Stamp, 1980; Hilker and Fatouros, 2015; Desurmont and Weston, 2011).

Our understanding on the ecology and mechanisms of oviposition-induced plant responses is largely increasing (Hilker and Fatouros, 2015; Reymond, 2013; Hilker and Meiners, 2011; Pashalidou et al., 2015b; Little et al., 2007). However, some aspects of plant-insect egg interactions have remained largely unexplored such as (i) the ability of an herbivorous insect to counteract oviposition-induced plant responses, and (ii) the genotypic and individual variation of plant responses to insect oviposition. In contrast to these gaps in knowledge about plant-insect egg interactions, solid information is available on the variability of plant responses to feeding arthropods. Genotype-based plasticity of feeding-inducible plant defensive responses is well documented (Stam et al., 2014; Degen et al., 2004; Gols et al., 2008a; Gols et al., 2009; Gols et al., 2008b; Kappers et al., 2011) and has been suggested to be shaped by the costs of this plasticity (Agrawal, 2002). Furthermore, individual variation in the composition of plant defence-eliciting compounds that feeding larvae introduce into plant wounds is well known (Roda et al., 2004), and some means of counteractions of larvae against feeding- 
induced plant defences have been detected (Musser et al., 2002; Lawrence et al., 2008; Lawrence et al., 2007; Weech et al., 2008; Bos et al., 2010; Consales et al., 2012; Chung et al., 2013).

Here, we address the above-mentioned knowledge gaps in plant-insect egg interactions by studying the response of various accessions of the black mustard Brassica nigra to egg deposition by the large cabbage white butterfly (Pieris brassicae). The annual B. nigra shows diverse responses to eggs of Pieris spp., including accelerated flower and seed production as reproductive escape strategy (Lucas-Barbosa et al., 2013; Pashalidou et al., 2013), attraction of egg- and larval parasitoids (Fatouros et al., 2014; Pashalidou et al., 2013; Fatouros et al., 2012; Cusumano et al., 2015; Pashalidou et al., 2015c), phenotypic changes that affect subsequent herbivore and parasitoid preferences and performances (Pashalidou et al., 2015a; Pashalidou et al., 2015b; Pashalidou et al., 2015c), and HR-like necrosis (Shapiro and DeVay, 1987; Fatouros et al., 2014; Pashalidou et al., 2015a; Fatouros et al., 2012). Different populations of B. nigra respond at different frequencies to singly laid $P$. rapae eggs or to clustered $P$. brassicae eggs by HR-like necrosis (Fatouros et al., 2014; Fatouros et al., 2012). Under both greenhouse and natural conditions, the hatching success of larvae from solitary P. rapae eggs was reduced when the plant expressed HR (Fatouros et al., 2014).

For the gregarious $P$. brassicae, which lays its eggs in clusters, it is unknown as yet whether formation of egg-induced HR-like symptoms and the hatching success depend on the number of eggs per cluster. Although effects of HR-like necrosis on $P$. brassicae egg survival were previously studied under greenhouse conditions (Fatouros et al., 2012), a thorough study examining the relationship between HR and survival of clustered eggs of $P$. brassicae under natural conditions is still lacking. To date, hardly anything is known about the ecological determinants of egg responses induced by herbivore oviposition. As shown in previous studies, egg load (Desurmont et al., 2011) and abiotic factors such as temperature or water stress (Shapiro and DeVay, 1987; Petzold-Maxwell et al., 2011; Salerno et al., 2017) can negatively or positively affect egg-induced plant defences.

In the present study, we investigated the following specific questions by conducting field, greenhouse, and laboratory experiments: Does formation of HR-like symptoms by B. nigra in response to $P$. brassicae egg deposition differ (1) among plant accessions and (2) among individuals of a B. nigra accession? (3) Is mortality of clustered P. brassicae 
eggs dependent on the occurrence of HR and correlated with HR severity? (4) Does clustering of $P$. brassicae eggs counteract the plant's HR? Since abiotic environmental conditions may significantly impact on phenotypic plant traits, we also investigated how (5) abiotic parameters (temperature, humidity, total radiation and sum of rain) affect the expression of the plant's HR and mortality of butterfly eggs.

\section{Results}

Variation in HR among plant accessions: field data. We used a common-garden setting to investigate whether four self-fertilised accessions of $B$. nigra show differences in expression of HR induced by $P$. brassicae eggs. Expression of HR was significantly influenced by the plant accession receiving the eggs (GLM, $\chi^{2}=24.74, \mathrm{df}=3, P<0.001$, Fig. 2.1A). Accessions SF25 and SF48 had a higher proportion of plants expressing HR than accession SF19. Moreover, HR severity was also significantly affected by plant accession (GLM, $\chi^{2}=69.32$, df $=9, P<0.001$, Fig. 2.1B). HR severity of SF48 was significantly higher than HR severity of the three other plant accessions (Fig. 2.1B). We conducted this experiment in two consecutive years. The year of the experiment did neither influence the fraction of plants expressing HR, nor the HR severity for SF19 and SF48, which were tested in both years (GLM, $\chi^{2}=0.75, \mathrm{df}=1, P=0.39$ and GLM, $\chi^{2}$ $=2.06, \mathrm{df}=1, P=0.15$, respectively, Fig. 2.1$)$. In conclusion, some genotypes show a greater inducibility and severity of HR-like symptoms by $P$. brassicae eggs than others.

Variation in HR among plant accessions and plant individuals: greenhouse data. In a greenhouse experiment we investigated the variation in expression of HR with respect to plant accession by examining 38 plant individuals belonging to two genotypes which were exposed to eight to ten butterflies that each laid five eggs.

Variation among plant accessions. Unlike under field conditions, neither the presence/absence of HR symptoms nor HR severity were significantly different between the two plant accessions SF19 and SF48 (GLM: $\chi^{2}=0.19, \mathrm{df}=1, P=0.66$ and $\chi^{2}=2.94, \mathrm{df}=$ $2, P=0.23)$.

Variation among individual plants. Both the presence of HR symptoms in response to individual egg clutches and HR severity varied significantly among plant individuals (GLM: $\chi^{2}=196.59$, df $=37, P<0.001$ and $\chi^{2}=352.27$, df $=74, P<0.001$ ). As seen 
Chapter 2 Egg killing leaf necrosis of plant genotypes

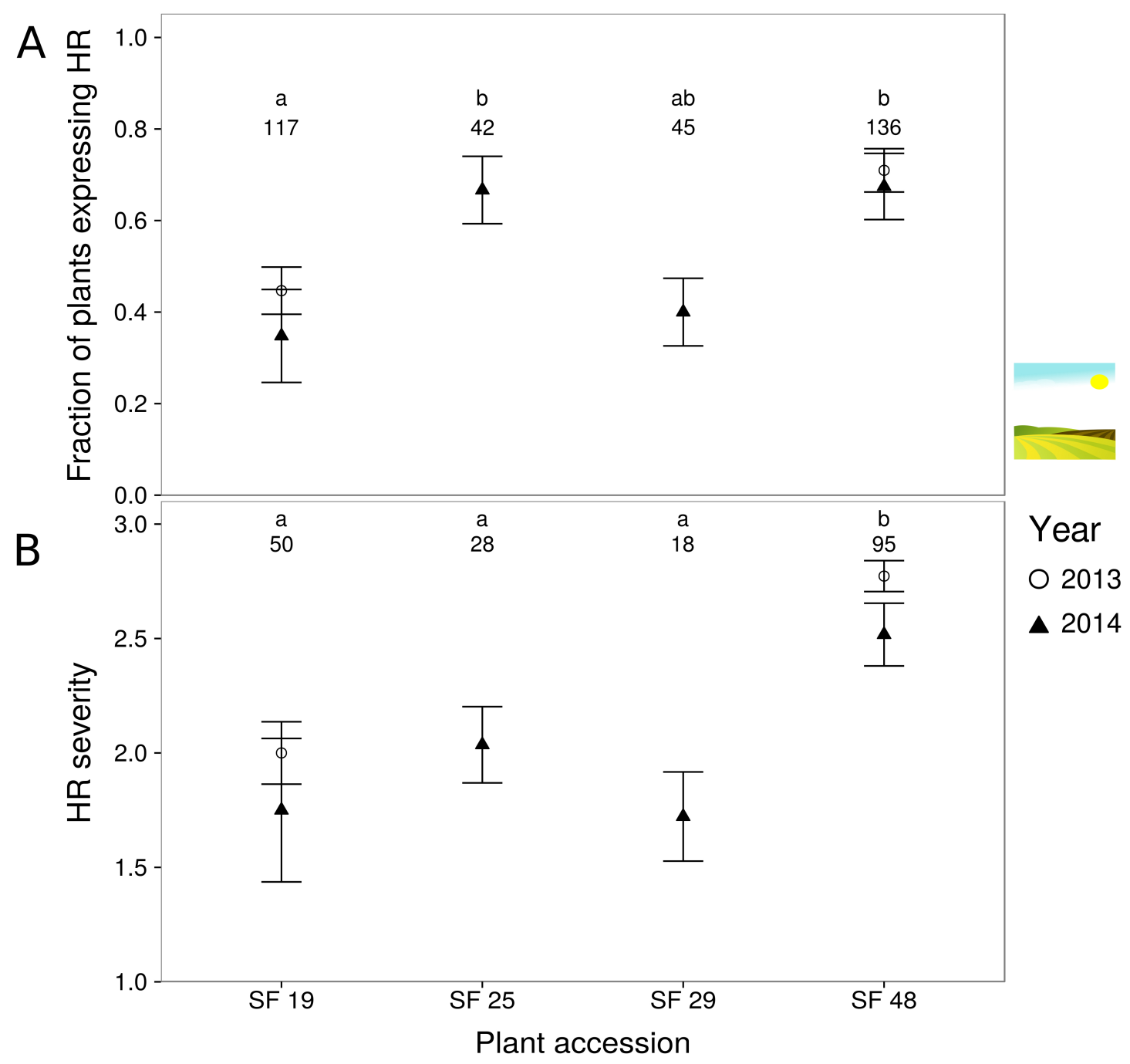

Figure 2.1: HR induced by $P$. brassciae eggs in B. nigra plant accessions used in common garden experiments in 2013 (only SF19 and SF48) and 2014. (A) Fraction of plants expressing HR (mean \pm SE) per plant accession. (B) HR severity (mean \pm SE) per plant accession (severity 1-3, compare Fig. 2.A.1). Numbers of plants tested for each accession are indicated above each data point, different letters indicate significant differences $(P<0.01$, LHT post hoc test). 
in Fig. 2.2A except for one plant (II.03), all plants expressed HR at least for some of the egg clutches. Seventeen plants expressed HR in response to all egg clutches laid onto them. With respect to the severity of the expressed HR about half of the plants $(\mathrm{N}=$ 18) expressed the lowest HR severity in response to all deposited egg clusters, one plant always the medium severity and two always the strongest. All other plants expressed varying degrees of HR severity for the egg clutches laid onto them (Fig. 2.2B). In conclusion, the data suggest that HR severity (and inducibility) is strongly linked to the individual plant, and differs only slightly between different egg clutches on the same plant. 
Chapter 2 Egg killing leaf necrosis of plant genotypes

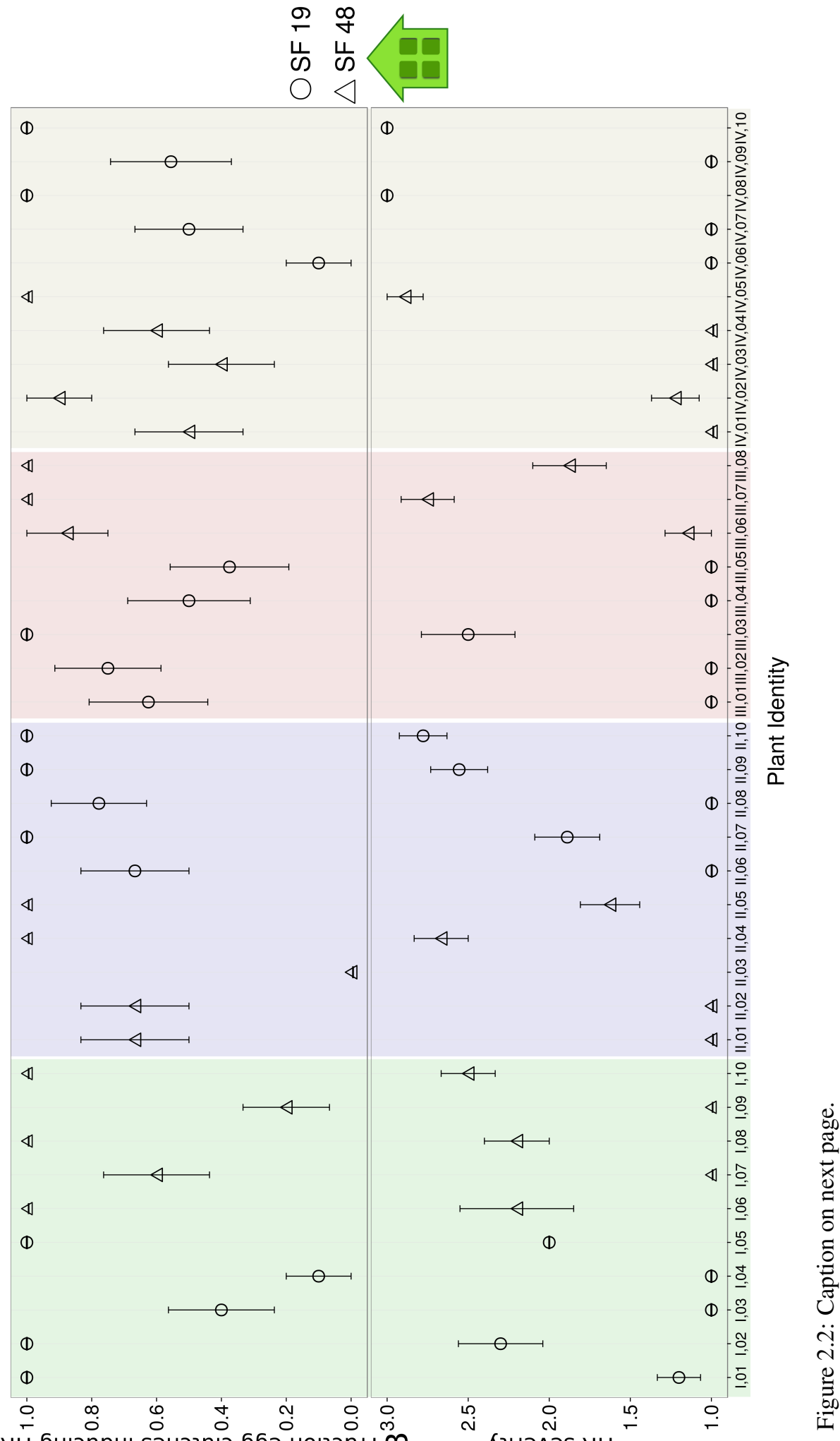


Figure 2.2: (Previous page.) HR induced by P. brassicae eggs in different plant individuals from two accessions under greenhouse conditions. (A) Fraction of egg clutches expressing HR per plant (mean $\pm \mathrm{SD}$ ). (B) Severity of HR when HR is expressed in plants (mean $\pm \mathrm{SD}$ ). Four different replicates and therefore plant/butterfly sets were used and marked with differently shaded background and (Latin) numbers for plant identity. Both plant accessions, SF19 and SF48 are separated using different shapes for data points. (see explanation in the figure).

Effects of HR on survival of clustered eggs: field and greenhouse data. Brassica nigra plants were infested with egg clutches of $P$. brassicae females either in the greenhouse or outside in a field experiment. Survival of egg clutches did not depend on the expression of HR (GLM, $\chi^{2}=0.19$, df $=1, P=0.66$ ), neither under greenhouse nor field conditions (GLM, $\chi^{2}=0.17$, df $=1, P=0.67$ and GLM, $\chi^{2}=0.01$, df $=1, P=$ 0.93 , respectively, Fig. 2.3A). Yet, egg survival was significantly greater in the greenhouse than in the field (GLM, $\chi^{2}=593.34$, df $\left.=1, P<0.001\right)$. Moreover, under field conditions, survival rates were significantly higher in 2013 than in 2014 (GLM, $\chi^{2}=$ 10.72 , $\mathrm{df}=1, P=0.001$ ). The large difference in egg survival rates between the greenhouse (almost 100\% survival) and field (between 35 and 50\% survival) could be due to abiotic factors as well as biotic factors, such as egg predation/parasitism. When egg clutches were protected by fine meshed nets from parasitoids and predators in the field, egg survival indeed significantly increased (from $33 \%$ to $60 \%$ in 2013, and $19 \%$ to $51 \%$ in 2014) in both years (MWU, 2013: $\mathrm{W}=2700.5, P<0.001$ and 2014: $\mathrm{W}=1256, P$ $<0.001$, respectively, Fig. 2.3B. This suggests that HR is not an effective defence trait against clustered $P$. brassicae eggs.

Effects of HR on survival of single versus clustered eggs. We investigated formation of HR in dependence of the egg clutch size (number of eggs) per plant and recorded egg survival in dependence of whether eggs were laid singly or clustered. Singly laid eggs were experimentally achieved by manipulating the female's oviposition mode.

Under greenhouse conditions, HR negatively affected survival of a single egg per plant (GLM, $\chi^{2}=6.35$, df $=1, P=0.01$, Fig. 2.4A). However, when more eggs were laid per plant and when these were laid in small $(\mathrm{N}=10)$ or larger groups $(\mathrm{N}=50$ or 90), the expression of HR did not affect egg survival (GLM, small clutch size/number of eggs: $\chi^{2}=1.80, \mathrm{df}=1, P=0.18$, medium: $\chi^{2}=0.83$, $\mathrm{df}=1, P=0.36$, and large: 

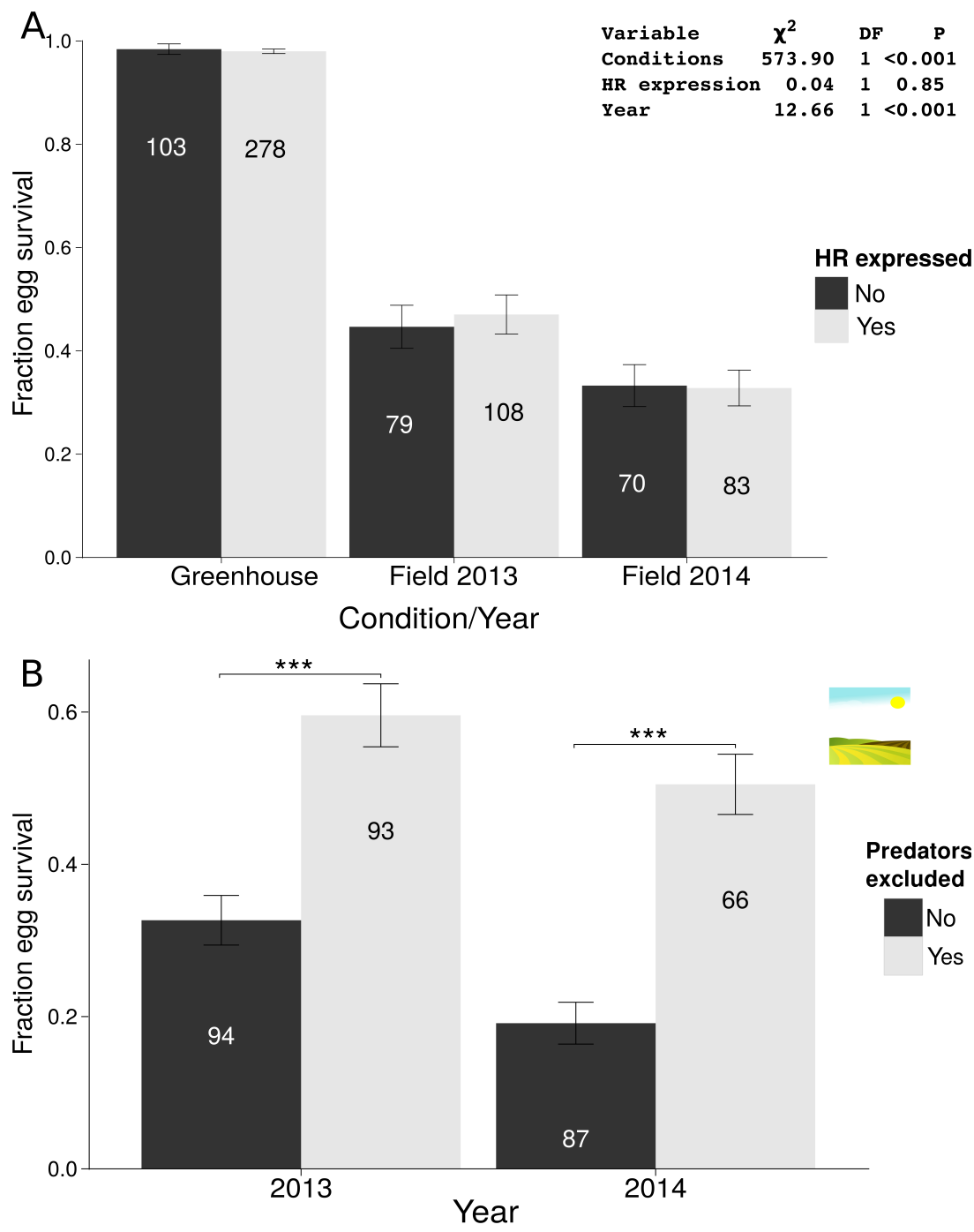

Figure 2.3: Effect of HR expression on survival of clustered $P$. brassicae eggs (mean $\pm \mathrm{SE}$ ) laid on B. nigra plants under greenhouse and field conditions (for 2013 and 2014). (A) The numbers within each bar represent the numbers of tested plants. The differences in egg survival until larval hatching between HR expressing (light grey) and non-HR expressing plants (dark grey) are not significant, GLM, family = quasibinomial. Differences between greenhouse and field conditions and between both years of field experiments are indicated by asterisks above the horizontal lines, GLM, family = quasibinomial. (B) Effect of predator/parasitoid exclusion on survival of clustered $P$. brassicae eggs (mean fraction $\pm \mathrm{SE}$ ) laid on B. nigra plants in the field. Plants were either covered with a fine mesh during the egg phase (predators excluded - light grey) or left uncovered (predators included dark grey). 
$\chi^{2}=1.40, \mathrm{df}=1, P=0.24$, Fig. 2.4A), while HR negatively affected survival when a female was forced to lay a single egg (GLM, $\chi^{2}=6.35$, df $=1, P=0.01$, Fig. 2.4A).

In a second experiment, we focused on the question how HR affects egg survival if the number of eggs is the same per plant (here 10 eggs per plant), but the type of egg distribution on a plant differs (singly laid eggs vs clustered eggs). Egg survival was significantly lower on plants expressing HR than on plants that did not express HR (GLM, $\chi^{2}=5.25$, df $=1, P=0.02$, Fig. 2.4B). The egg distribution (10 singly laid eggs vs. 10 eggs laid in a cluster) and the interaction between HR expression and egg distribution did not affect egg survival (GLM, $\chi^{2}=1.73$, df $=1, P=0.19$ and $\chi^{2}=$ 0.34 , df $=1, P=0.56$, Fig. 2.4B). However, when the effect of egg distribution on egg survival was tested separately, egg survival was only lower when HR was induced by singly laid eggs (GLM, $\chi^{2}=4.07, \mathrm{df}=1, P=0.04$, Fig. 2.4B) and not by a clutch of 10 eggs (GLM, $\chi^{2}=1.99$, df $=1, P=0.16$, Fig. 2.4B).

Moreover, both in the greenhouse and in the field, the number of eggs per plant did not affect whether HR was expressed or not. Under greenhouse conditions no significant differences were found between plants with varying numbers of eggs (a single egg, groups of 10, ca. 50, ca. 90) in their probability of HR expression (GLM, $\chi^{2}=0.001$, df $=1, P=0.98)$ or the severity of the HR expressed (GLM, $\chi^{2}=0.313, \mathrm{df}=1, P$ $=0.58$, Fig. 2.5A). Under field conditions no significant differences in probability of HR expression were found between plants induced by eggs laid in different clutch sizes (GLM, $\chi^{2}=0.003, \mathrm{df}=1, P=0.96$ ). For the severity of expressed HR no significant differences were found either (GLM, $\chi^{2}=3.45$, df $=1, P=0.06$, Fig. 2.5B). However, in the field one genotype (SF29) showed a significantly higher probability to express HR in response to medium-sized egg clutches compared with small ones (Supplementary Figure 2.A.1).

To conclude, the results suggest that HR is an effective defence strategy against single eggs, rather than clustered eggs and that plants do not increase expression or severity of HR with an increasing number of eggs laid. 

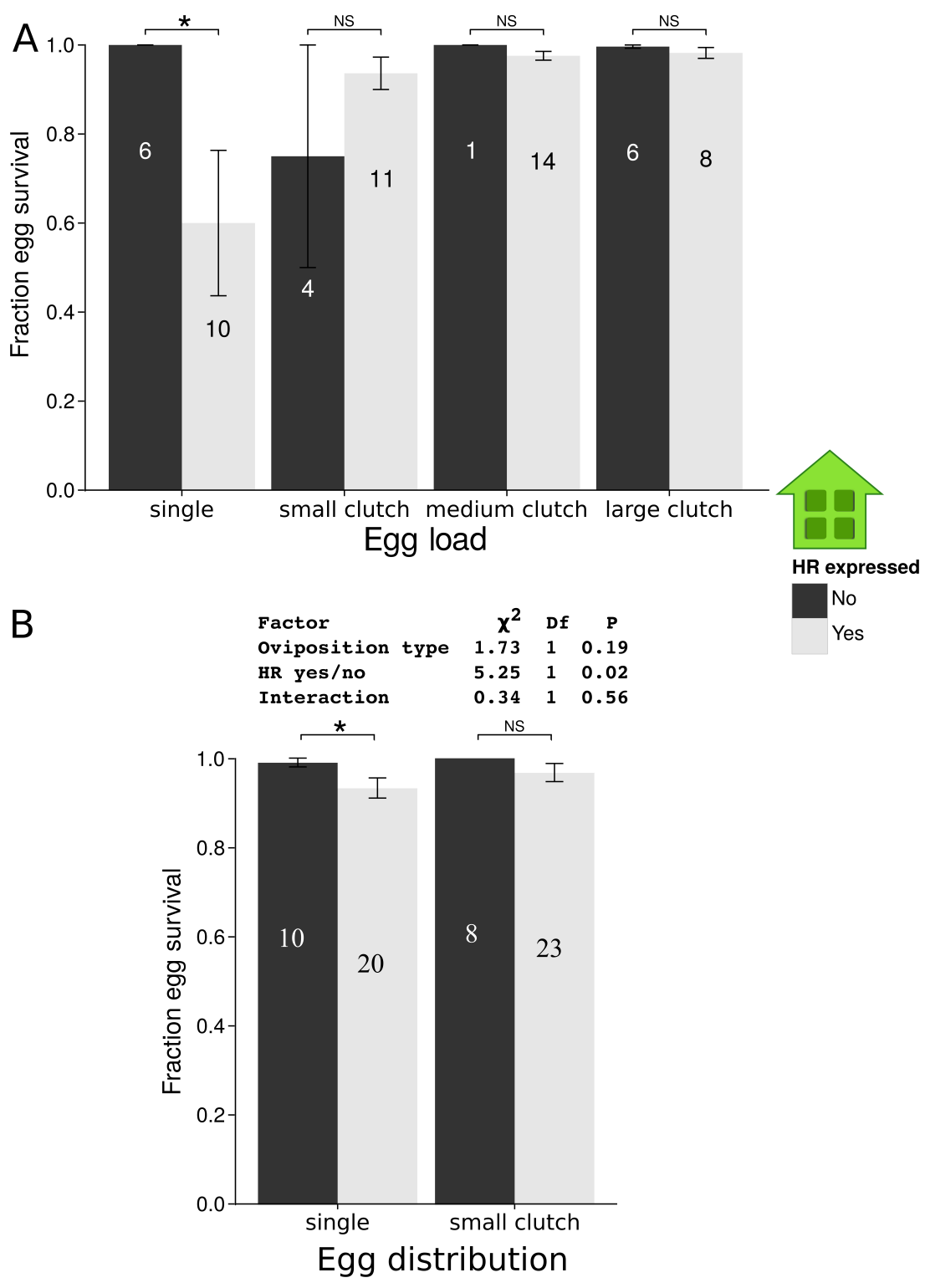

Figure 2.4: Fraction of $P$. brassicae egg survival under greenhouse conditions. (A) Impact of number of eggs per plant and egg distribution. Effect of HR expression on survival (mean fraction \pm SE) of single $P$. brassicae eggs per plant and $P$. brassicae egg clusters of different size as stated on the X-axis (small: 10, medium: 50 and large: 90 eggs) laid on B. nigra plants. Survival rates of $P$. brassicae eggs on B. nigra plants expressing HR (light grey columns) or not (dark grey columns). Numbers in the columns represent the numbers of tested plants. (B) Impact of egg distribution. Effect of HR expression on survival (mean fraction \pm SE) of 10 singly laid $P$. brassicae eggs and of a $P$. brassicae egg cluster (containing 10 eggs) on $B$. nigra plants under greenhouse conditions. Survival rates of eggs on $B$. nigra plants expressing HR (light grey columns) or not (dark grey columns). Numbers in the columns represent the numbers of tested plants. Significant differences are indicated using asterisks. ${ }^{*} P<0.05$, ns: not significant, GLM. 


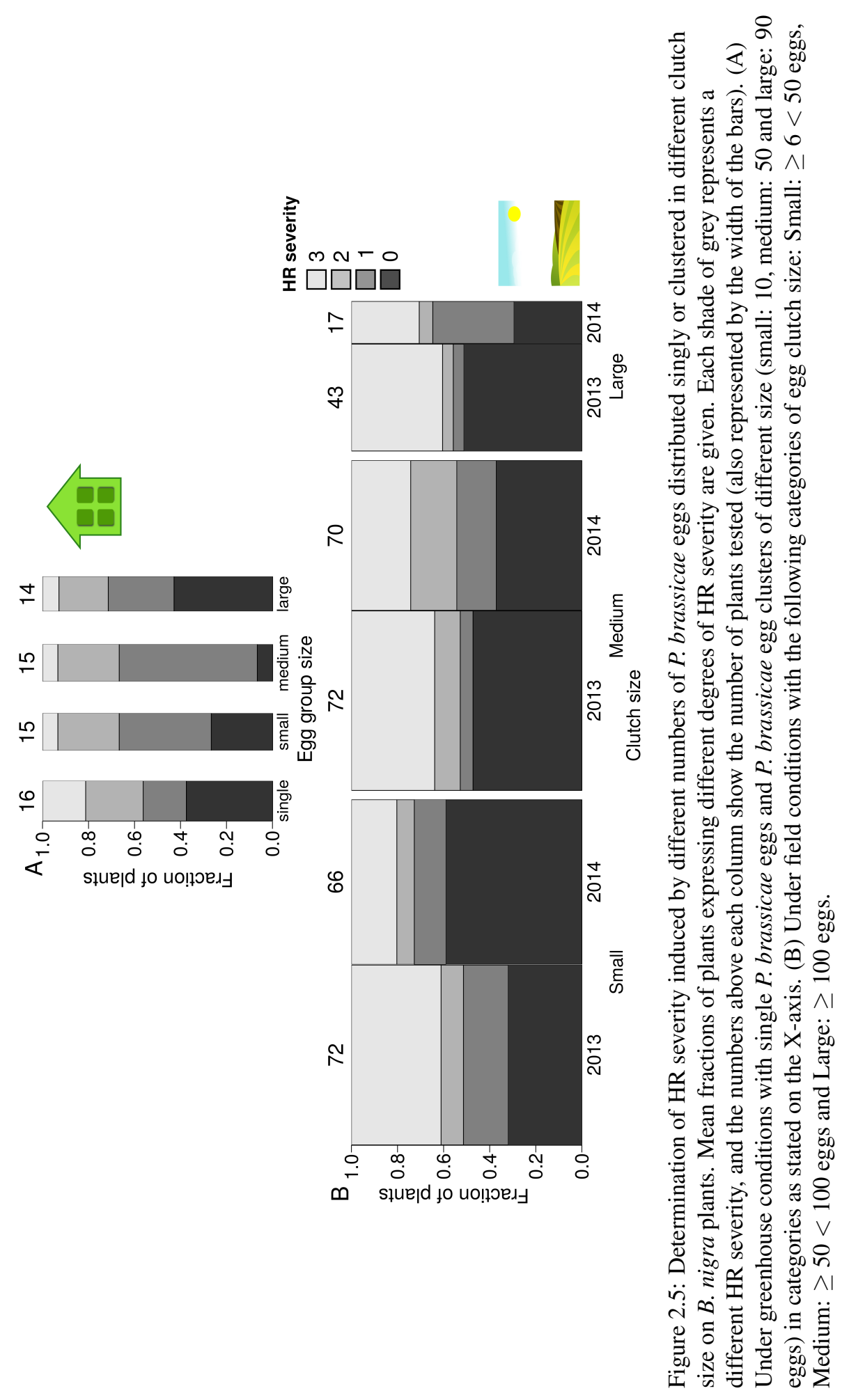


Effects of desiccation on egg survival. Since humidity conditions at necrotic leaf tissue are expected to differ from those at live, transpiring tissue, we tested how survival of Pieris eggs depends (i) on humidity and (ii) on whether eggs were laid singly or in a clutch (with five eggs).

Overall, eggs in groups of five showed higher survival rates than single eggs (GLM, $\chi^{2}=12.38$, $\left.\mathrm{df}=1, P<0.001\right)$. The humidity conditions applied here significantly affected egg survival (GLM, $\chi^{2}=47.07$, df $=3, P<0.001$, Fig. 6), while the interaction between humidity and egg group/singly laid egg did not (GLM, $\chi^{2}=2.68$, df $=3, P=$ $0.44)$.

When considering egg survival rates at distinct humidity conditions, however, eggs in clutches of five showed a significantly higher survival rate for the treatment 'low - high RH' (GLM, $\left.\chi^{2}=10.45, \mathrm{df}=1, P=0.001\right)$, but not for the 'min RH' treatment (GLM, $\chi^{2}$ $=3.02$, df $=1, P=0.08$ ), 'high - low RH' (GLM, $\chi^{2}=0.99$, df $\left.=1, P=0.32\right)$ or max RH treatment (GLM, $\chi^{2}=0.60, \mathrm{df}=1, P=0.44$, Fig. 2.6). The min $\mathrm{RH}$ treatment resulted in a significantly lower survival of eggs laid in a clutch compared to all other water treatments (Fig. 2.6). On the other hand, survival of singly laid eggs was significantly lower when no water was added in the beginning of the experiment (min $\mathrm{RH}$ and low - high RH treatments, Fig. 2.6). The weight loss/gain of eggs was different between single and clustered eggs. Single eggs lost or gained weight faster than the clustered eggs (Supplementary Figure 2.A.2). However, these differences were only significant for one time period in the high to low humidity treatment (between $51.625 \mathrm{~h}$ and $70.25 \mathrm{~h}$ after starting, Tukey HSD, $P=0.003$, Fig. 2.A.2) and at two time periods in the low to high treatment (between $3.125 \mathrm{~h}$ and $22.875 \mathrm{~h}$ after starting, Tukey HSD, $P=0.001$ and between $51.625 \mathrm{~h}$ and $70.25 \mathrm{~h}$ after starting, Tukey HSD, $P<0.001$, Supplementary Figure 2.A.2). The weight fluctuations are probably due to the loss and uptake of water and/or respiration. The stronger weight fluctuation in single eggs exposed to changing humidity conditions might explain their lower survival rates.

Effects of abiotic factors. None of the weather factors (sum daily rainfall, relative humidity, mean daily temperature and mean total daily radiation) was correlated with the fraction of plants expressing HR in both experimental field seasons. None of the parameters had a significant impact on the fraction of egg clutches inducing HR (GLM, for (a) $\chi^{2}=0.84, \mathrm{df}=1, P=0.36$, (b) $\chi^{2}<0.001$, df $=1, P=0.98$, (c) $\chi^{2}=0.01$, df 


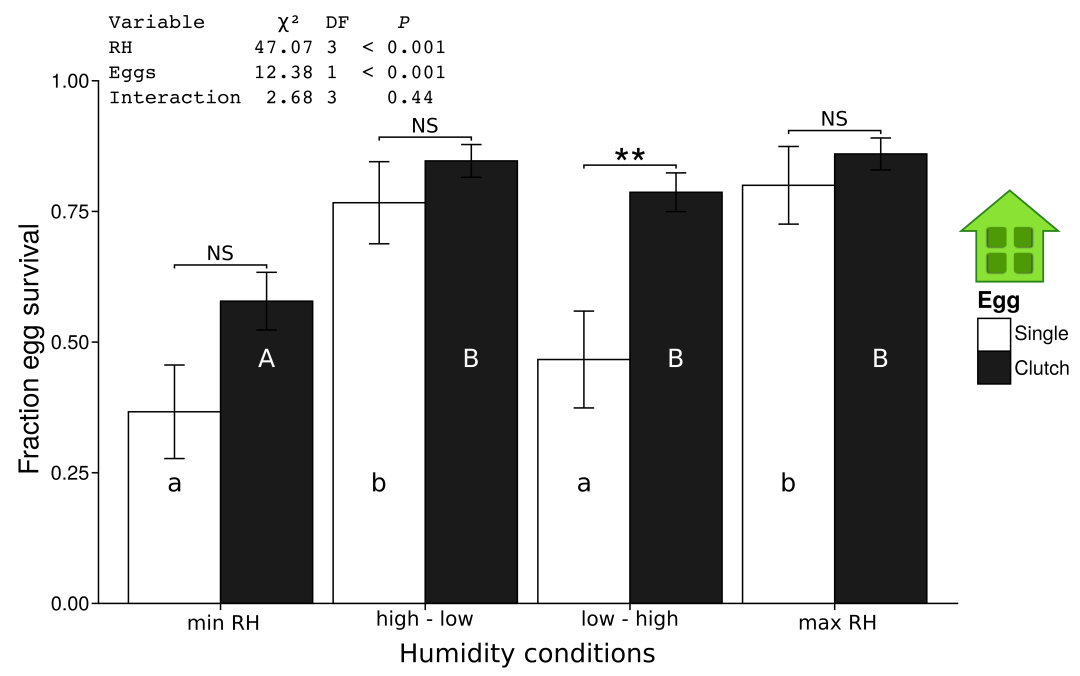

Figure 2.6: Effect of various humidity conditions on survival of $P$. brassicae eggs (mean fraction \pm SE) until larval hatching. For the water/humidity treatment: compare Fig. S8. Different lowercase letters indicate significant differences in survival $(P<0.05$, LHT post hoc test) of single eggs kept at different humidity conditions, while capital letters indicate significant differences in survival rates $(P<0.05$, LHT post hoc test) of five eggs (clutch) kept at different humidity conditions. Asterisks above bars indicate whether or not survival rates of eggs differ between single and clustered eggs kept at a particular humidity treatment (LHT post hoc test). NS = Not significant, $* *$ $P<0.01$, GLM. N per treatment $=30$.

$=1, P=0.91$ and (d) $\chi^{2}=0.82, \mathrm{df}=1, P=0.37$, Supplementary Figure 2.A.3). Egg survival was tested under the above-mentioned weather factors, the expression of HR and the interaction between the weather factor and HR expression. Mean sum of the daily rainfall did significantly influence egg survival (GLM, $\chi^{2}=4.95$, df $=1, P=0.03$, $\chi^{2}=0.09$, Supplementary Figure 2.A.4) Interestingly, survival rates were highest when rainfall was moderate, indicating again that higher air humidity benefits egg survival, while highest rainfall brought the egg survival back to the same level as low rainfall. Neither HR expression nor the interaction of both changed egg survival (GLM, $\mathrm{df}=1$, $P=0.77, \chi^{2}=0.28, \mathrm{df}=1, P=0.60$, Supplementary Figure 2.A.4). None of the other factors significantly affected egg survival (Supplementary information).

\section{Discussion}

Our study reveals that plant genetic background influenced the likelihood and severity of HR induced by $P$. brassicae eggs under natural conditions. Interestingly, this variation in 
an egg-killing plant defence trait was not effective against a butterfly that deposits eggs in clusters. We show that the larval hatching rate from P. brassicae eggs laid in clutches of different sizes on B. nigra plants expressing HR-like necrosis is not affected by clutch size, neither under greenhouse nor under natural conditions. Only when butterflies were forced to lay single eggs, egg survival was lower when leaf necrosis was induced. Our data show that singly laid eggs suffer a stronger decrease in survival under low humidity conditions than eggs deposited in groups, which could explain the differences in the detrimental effect of HR-like necrosis on single and clustered eggs.

We hypothesize that formation of HR necrosis evolved as a defensive trait against lepidopteran specialists of brassicaceous plants. Two tested generalist moth species, Spodoptera exigua and Mamestra brassicae did not induce HR necrosis in B. nigra (Pashalidou et al., 2013; Fatouros et al., 2012; Cusumano et al., 2015). A cue common to egg deposition by pierid species might trigger HR. Pieris brassicae is the only pierid species in The Netherlands that clusters eggs on brassicaceous plants (Lewinington, 2016). This oviposition behaviour might render HR-like necrosis useless as a defence against eggs in this specific case.

An attenuating effect of egg clustering on the influence of an induced plant defensive response was also shown for the interaction between Viburnum plant and eggs of the viburnum leaf beetle (Pyrrhalta viburni): the beetle females prefer to oviposit on egg-infested Viburnum twigs by inserting their eggs into a cavity which the gravid female gnaws into the twig (Desurmont and Weston, 2011; Hilker, 1992). Egg deposition induces the twig to grow wound tissue which squeezes the eggs. When eggs are aggregated in higher numbers, this egg-induced response is mitigated (Desurmont and Weston, 2011).

Even though egg clustering seems very effective to counteract inducible egg-killing defences, it may increase the risk of parasitism and predation. Egg clusters might be easier detectable by visually orienting predators like birds than singly laid eggs. Furthermore, insect egg clusters might also be attractive targets for small egg parasitoids like Trichogramma spp. as they can easily parasitise many eggs after having encountered a clutch (Fatouros and Huigens, 2012; Huigens and Fatouros, 2013). Besides possible predation or parasitism risks, cannibalism is common in gregarious species. Upon hatching, P. brassicae caterpillars both eat their own egg shell and sometimes cannibalise the eggs of their siblings (Clark and Faeth, 1998) (E. Griese, pers. obs.). Finally, egg cluster- 
ing may lead to competition for food among the caterpillars if the resources are limited. Some or all of these factors might have led to the situation where most species belonging to the Pierini such as P. rapae lay single eggs despite the fact that egg clustering can protect eggs from desiccation due to HR-like necrosis.

From the plant's perspective, successful egg-killing defences reduce the chance of larval feeding damage. Gregariously feeding $P$. brassicae larvae can completely defoliate a B. nigra plant and cause irreversible damage to flowers (E. Griese \& D. Lucas-Barbosa pers. obs.). In a previous study, it was shown for the solitary $P$. rapae that the percentage of eggs inducing HR is not dependent on the number of singly laid eggs on the plant, ranging from 2 to 9 . However, formation of strong HR resulted in significantly reduced survival of the singly laid $P$. rapae eggs (Fatouros et al., 2014). For Heliconius subflexa, the survival of singly laid eggs on Physalis sp. plants was lower when HR and/or neoplasm formation were expressed (Petzold-Maxwell et al., 2011). These examples corroborate our observation that singly deposited eggs of butterflies are affected by egg-killing plant responses.

Because plants will suffer more feeding damage from larvae hatching from egg clutches than from a few singly laid eggs, plants likely benefit from expressing a stronger HRlike necrosis against a higher number of eggs laid in a cluster. However, we did not find a correlation between the number of eggs laid (in a single clutch) and the probability or strength of HR formation. Moreover, the hatching success of P. brassicae larvae was not reduced by HR. Only when P. brassicae butterflies were forced to lay eggs singly onto the plant, the expression of HR reduced the hatching success. Our data indicate that $P$. brassicae can benefit from laying eggs in clutches. So far, it has been suggested that egg clustering increases the reproductive success of female butterflies (Courtney, 1984), especially when host plants are scarce. Hence, egg clustering may be beneficial for $P$. brassicae for two reasons: on the one hand it may be a cost-saving behaviour with respect to the energy invested in searching for oviposition sites, and on the other hand it obviously counteracts the negative effects of an egg-inducible direct plant defence trait, i.e. HR-like leaf necrosis.

The present study reveals that plant accessions differ in HR-inducibility (likelihood of expressing HR) and the severity of HR expressed in response to insect egg deposition. Previous studies have shown genetic variation in plant defence traits only for defences against feeding herbivores (e.g. (Agrawal and van Zandt, 2003)), among them also labor- 
atory and field studies of Brassica species investigating defence traits against feeding Pieris larvae. For example, laboratory experiments revealed that differences in volatile blends of $B$. oleracea var. alba L . varieties induced by feeding $P$. brassicae caterpillars lead to differences in attraction of a larval parasitoid. The differential attractiveness of these plant varieties to larval parasitoids matched different parasitism rates of larvae feeding on different varieties in the field; furthermore, herbivore preference and performance, as well as insect communities living on the plants varied among these plant varieties (Poelman et al., 2009a; Poelman et al., 2009b; Poelman et al., 2010). These results are in line with our findings, confirming that different genotypes of a Brassica species differ in their feeding- and oviposition-inducibility of anti-herbivore defence traits under field conditions.

Our data further show variation among individual plants in HR expression. Some plant individuals exhibited resistance to eggs and expressed an HR-like response, whereas others did not. It is likely that this variation is caused by a high variation in the responsiveness of a resistance (R) gene in B. nigra plants recognising specific cues of Pieris eggs. R-genes targeting phytopathogens are among the most polymorphic loci in plant genomes (Karasov et al., 2014a; Karasov et al., 2014b). Whether this is similar for R-gene(s) involved in HR against Pieris eggs is an open question. Studies of plantpathogen coevolution often report one type of interaction, the gene-for-gene interaction, whereby plants generate $\mathrm{R}$ products that recognise cues from the specific attacker (Jones and Dangl, 2006). Although the plant genotypes used in our study originated from a selffertilisation event (produced by E.H. Poelman) (or crossing of two self-fertilised plants of the same genotype, produced by E.H. Poelman and E. Griese), they are not homozygous which may explain the observed variation in HR expression between individual plants of each accession.

Our data suggest that laying eggs in clusters reduces their susceptibility to HR-like necrosis. Formation of necrotic leaf tissue might lower the humidity around the egg clutch because of reduced plant transpiration at this site. Shapiro and DeVay (1987) suggest that HR kills eggs by reducing humidity at the interface between plant surface and eggs, thus resulting in detachment of eggs from the leaf or in egg desiccation. Water loss of eggs is determined by various factors. In addition to atmospheric humidity, transpiration of the plant tissue and other egg-related factors like the chemical composition of the eggshell, the serosa, size of the egg surface as well as the exocrine secretion 
attached to the eggshell may play a role in preventing egg desiccation (Hinton, 1981; Margaritis, 1985; Woods, 2010). Especially the formation of the serosa has been shown to drastically increase drought resistance in eggs of several insect species, including beetles and mosquito species (Rezende et al., 2008; Goltsev et al., 2009; Jacobs et al., 2013). Whether the serosa plays a similar role in butterfly eggs remains to be investigated. In addition to the mentioned parameters, aggregation of eggs could prevent desiccation under low humidity conditions, as was shown for Chlosyne lacinia butterflies feeding on the common sunflower Helianthus annuus (Clark and Faeth, 1998). Their egg clutches can vary in size and the number of layers. A higher number of egg layers and larger clutch size improved the hatching success under low humidity conditions (Clark and Faeth, 1998). In the present study, we show that clustered P. brassicae eggs can recover better from low humidity conditions when transferred to high humidity than singly laid eggs; the survival rate of clustered eggs was higher under these conditions. This indicates that the strong, negative effects of HR-related necrosis on especially the singly laid eggs might also be due to reduced humidity at the oviposition site. Thus, by implication, egg aggregation can protect eggs against a detrimental HR-associated effect, i.e. low humidity at the oviposition site.

Unlike under field conditions, the different accessions did neither differ in the likelihood of expressing HR, nor in the strength of HR expression when placed under greenhouse conditions. Furthermore, the proportion of plants that show HR as well as the average HR severity were generally lower. These results suggest that abiotic factors present in the field but not in the greenhouse (e.g. UV-light or higher temperature and humidity fluctuations), affect HR expression. However, in our field experiment, none of the particular abiotic factors that we determined affected the expression of $\mathrm{HR}$ in B. nigra in neither of the two seasons. Nevertheless, some plant accessions might have been more affected by changes in abiotic conditions in the field than others, which would explain the more similar HR expression of the accessions in the greenhouse. In contrast to our experimental field data, Petzold-Maxwell et al. (2011) showed that temperature was positively correlated with the likelihood of the expression of HR and neoplasm formation induced by eggs of the moth Heliothis subflexa in Physalis angulata plants in a field experiment; the temperatures measured in this study were higher than the highest temperature measured in our field study (ca. $21^{\circ} \mathrm{C}$ ). Furthermore, a previous study on B. nigra and P. rapae in California, U.S.A., indicated that HR necrosis was more pro- 
nounced at high temperatures (above $30^{\circ} \mathrm{C}$ ) (Shapiro and DeVay, 1987). The range of changes of abiotic factors as well as very high temperature and low humidity may affect the expression of HR.

Our study revealed that aggregating eggs is a strategy for $P$. brassicae to avoid negative effects of HR as a direct egg-killing response. However, there must be some fitness costs associated with egg clustering, because other common Pieris species (e.g. P. rapae and $P$. napi) lay single eggs (Wiklund, 1984). Costs of egg clustering could be associated with increased larval competition on the same food source and/or increased egg and larval predation or parasitism risks. Future studies need to elucidate the ecological consequences that $B$. nigra plant genotypes and individuals face when not expressing HR-like necrosis in response to $P$. brassicae eggs.

\section{Material and Methods}

Plants and insects. Brassica nigra L. plants were grown either in a greenhouse (18 $\left.\pm 5^{\circ} \mathrm{C}, 50-70 \% \mathrm{RH}, \mathrm{LD} 16: 8\right)$ or, for field experiments, first grown in the greenhouse for ca. one week, and then placed in an outdoor area protected from wind and rain for two to three weeks. For the experiments in 2013, we used seeds of two self-fertilised plants (SF19 and SF48) that had been collected in 2009 from a B. nigra population near the River Rhine in Wageningen, The Netherlands $\left(51.96^{\circ} \mathrm{N}, 5.68^{\circ} \mathrm{E}\right)$. In 2014 , seeds produced from plants of the original self-fertilised plants of the same accessions- and of two additional accessions were used (SF19, SF25, SF29 and SF48).

Pieris brassicae L. (Lepidoptera: Pieridae) was reared on Brussels sprouts plants ( $B$. oleracea var. gemmifera cv. Cyrus $)$ in a climate room $\left(21 \pm 1{ }^{\circ} \mathrm{C}, 50-70 \% \mathrm{RH}, \mathrm{LD}\right.$ 16:8). Freshly emerged virgin female and male P. brassicae butterflies were obtained from the rearing and kept isolated. Two days after emergence they were allowed to mate, and two days later the mated female butterflies were used for egg deposition in the experiments.

Experimental conditions. Common garden experiments under field conditions. Research questions 1, 3 and 5 were tested in a common garden setup, conducted in two consecutive years (2013 and 2014). In both years, young greenhouse-grown seedlings (three weeks old) were planted into the field in three different periods, two weeks apart 
from each other, starting mid-May and ending end of July. The seedlings were planted in fields of the experimental farm of Wageningen University (Unifarm), The Netherlands. Several B. nigra accessions were planted in the field. In 2013, SF19 and SF48 were grown, and in 2014 we planted SF19, SF25, SF29 and SF48. In total 192 plants in 2013 and 240 in 2014 were infested with $P$. brassicae eggs. The plants were organised into plots namely in 12 rows of four plots each in 2013 and in 15 rows of four plots each in 2014. Each plot contained four egg-infested plants with three metre distance between the plots. A mixture of Lolium and Poa grasses was sown in between the plots. The egg-infested plants were grown about two metre apart from each other within a plot. Half of the plants were covered with a fine mesh protecting eggs from predators and parasitoids. Plants were infested with eggs when they were four to five weeks old. One to two weeks after planting, each plant was exposed to a mated female which was kept on a plant until egg deposition (between 0.5 to $24 \mathrm{~h}$ ) by covering it with a gauze net.

After deposition of one egg cluster (additional clusters were gently removed), the leaf with the egg cluster was photographed for documentation and later counting of the eggs per cluster.

Greenhouse experiments. Research questions 1 to 4 were also addressed under greenhouse conditions $\left(22 \pm 2{ }^{\circ} \mathrm{C}, 50-70 \% \mathrm{RH}, \mathrm{LD} 16: 8\right)$.

Egg survival. The survival rate of eggs was calculated based on the total number of eggs and the number of caterpillars that hatched (hatched caterpillars/total number of eggs). The number of caterpillars was counted just after hatching by visual observation or by taking a picture and counting eggs and caterpillars with the help of the computer programmes ImageJ as described by Schneider et al. (2012) and Gimp (see http://www.gimp.org/).

Determination of HR-like necrosis. All plants in the field and greenhouse were visually checked for HR symptoms three to four days after egg deposition (Fig. 2.7). The plants were categorised into 'HR-' (no necrotic zone observed, Fig. 2.7A) or 'HR+' (with necrotic zone, Fig. 2.7B-D). When HR was observed, its severity was quantified by categorising it into three different classes: necrosis visible underneath the eggs (HR severity '1') (Fig. 2.7B), necrosis also visible on the other side of the leaf (HR severity '2') (Fig. 2.7C), and necrosis visible around the eggs and the other side of leaf with 
clear edges (HR severity ' 3 ') (Fig. 2.7D). Four days after egg deposition, the HR is fully expressed and does not change any more.

Effect of plant individual and genotype on HR expression. A four-week-old B. nigra plant from either the SF19 or SF48 accession was infested with eggs in the greenhouse as described above. A female butterfly was placed on a leaf for oviposition. As soon as it had laid five eggs, the butterfly was removed from the cage and a new female was introduced. After a plant had received eggs from eight to ten female individuals (five eggs per female), it was replaced by a new one and offered to the same set of butterflies. Hence, in total each plant received 40 to 50 eggs on a single leaf, a procedure which was repeated for eight to ten plants (half of them being SF19, half SF48) using the same set of females (Supplementary Figure 2.A.5). In total, four sets of eight to ten plants, each with eggs from a different set of eight to ten butterflies were prepared. The youngest leaf large enough to sustain all eggs (usually $3^{\text {rd }}$ or $4^{\text {th }}$ leaf from the top of the plant) was infested with eggs, and infestation took less than one hour per plant. The sequence of butterflies used for oviposition was randomised for each plant. We recorded HR-like symptoms and their severity induced by each egg cluster for a period of four days after egg deposition. Egg survival was recorded as well (as described above). Thus, these parameters were assessed for each plant individual.

\section{Effects of egg clutch size and egg clustering on HR expression and egg survival.}

To investigate whether the number of eggs laid affects HR severity under greenhouse conditions, $P$. brassicae butterflies were allowed to deposit eggs in three different clutch sizes on plants from two B. nigra accessions (SF19 and SF48). Four-week-old B. nigra plants were placed in a cage $(35 \times 60 \times 40 \mathrm{~cm})$ together with one $P$. brassicae female to obtain either 1, 10, 50 or 90 eggs per plant. Additional eggs were gently removed right after discovery using a soft brush. Fourteen to sixteen plants per treatment were infested.

To investigate further whether the type of egg distribution (singly laid eggs versus clustered eggs) impacts on the effects of HR on egg survival when plants are deposited with the same number of eggs, we conducted a further experiment in which $B$. nigra plants were always infested with 10 P. brassicae eggs. The eggs were either laid as a clutch or singly. The 10 singly laid eggs were at least $5 \mathrm{~mm}$ apart but still on the 


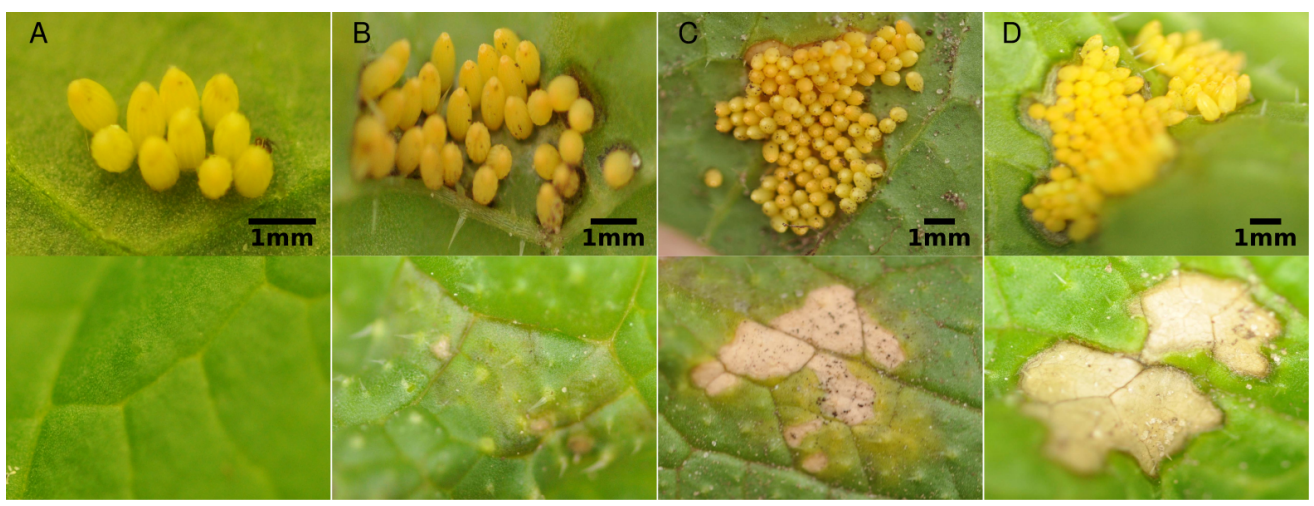

Figure 2.7: Different severity levels of HR-like necrosis induced by egg clutches of $P$. brassicae laid onto B. nigra plants under field conditions. The upper row shows the abaxial side of a leaf with eggs, the lower row shows the adaxial side of a leaf. (A) No HR-like necrosis induced (no HR = 0), (B) HR severity 1: necrosis can be observed below eggs, but hardly on the other side of the leaf, (C) HR severity 2: necrosis has spread and can be clearly observed on both sides of the leaf, the edges of the reaction are diffuse. (D) HR severity 3: necrosis is strongly expressed also around the egg clutch with sharp edges. All clutches shown are the same age (four days after egg deposition).

same leaf. For each egg distribution mode we used 32 plants. However, one female which was used for both egg distributions laid unfertilised eggs, and one egg-deposited leaf of a plant used for the single egg distribution broke off during the experiment, thus reducing the number of plants to 30 (for singly laid eggs) and 31 (for clustered eggs).

Under field conditions, we recorded HR-like symptoms and their severity induced by each egg cluster for a period of four days after egg deposition. We correlated the different egg clutch sizes with formation of HR and egg survival.

Effect of abiotic factors on HR expression and egg survival. Microclimate. Egginduced formation of necrotic leaf tissue is likely to be associated with changes in humidity at the interface between plant surface and eggs. To investigate whether differences in humidity and changes in humidity affect single and aggregated eggs differentially, a laboratory experiment was conducted in which freshly laid eggs were kept in a climate cell under controlled temperature and light conditions $\left(24 \pm 0.5^{\circ} \mathrm{C}, \mathrm{LD} 16: 8\right)$ but different humidity conditions (Supplementary Figure 2.A.6). First, a filter paper disc was laid into a Petri dish $(9.4 \mathrm{~cm}$ diameter), and lids of small Petri dishes $(5.8 \mathrm{~cm}$ diameter) were placed onto the filter paper discs. Eggs were carefully removed from $B$. oleracea var. gemmifera plants and transferred singly or in a group of five into the cap of an Eppendorf tube $(1.5 \mathrm{ml})$. The eggs had been laid maximally two hours before the 
experiment. Five caps with eggs were placed onto each small Petri dish lid. Then the large Petri dishes ( $9.4 \mathrm{~cm}$ diameter) were closed. The set up was chosen to avoid direct contact of eggs with the water. The following humidity conditions were generated: (i) The filter paper was completely soaked with $2 \mathrm{ml}$ water after placing the eggs into the dishes. We assumed that the relative humidity was constantly kept at nearly $100 \%$ under the closed lid during the whole egg-development period (4.5 days) (max RH treatment). (ii) A dry environment of the eggs (mimicking plant cell necrosis) was reached by not adding water (min RH treatment). (iii) The effect of change in humidity on egg survival was tested by adding $1 \mathrm{ml}$ water at the beginning of the experiment, and then allowing complete evaporation (humidity changes from high to low (referred to as high-low RH). (iv) The reverse treatment (humidity changes from low to high) was created by adding $1 \mathrm{ml}$ water only 2.5 days after placing the eggs into the setup (referred to as low-high $\mathrm{RH})$. The hatching success was measured after four to five days.

Macroclimate. Weather data for the periods in which the common garden experiments were conducted (see above) were obtained from the Veenkampen weather station of Wageningen University, around $3.25 \mathrm{~km}$ west of the field site. Data on the daily mean temperature $\left({ }^{\circ} \mathrm{C}\right)$, the sum of all net radiation per day $\left(\mathrm{W} \mathrm{m}^{-2}\right)$, mean relative humidity (\%) and the sum of daily rainfall were collected for all days of the experiment during the field season. Their values over the four days following egg deposition were used to calculate a mean $\pm \mathrm{SE}$ value. The mean value was checked for correlation with expression of HR and its severity. The mean value was also correlated with the mean value of egg survival for the period of time.

Statistical analysis. Generalised linear models (GLM) were used as the main tool for statistical analysis for all experiments. Egg survival and HR expression as response variable were analysed using a binominal distribution. The HR severity as response variable was analysed using a Poisson distribution. To compare different factor levels within a model pairwise Mann-Whitney-U tests were conducted. Alternatively, a Tukey HSD test was conducted if applicable. All tests were conducted using the statistic platform $\mathrm{R}$ 3.2.5 (R Core Team, 2014).

Data Availability. The datasets generated during and/or analysed during the current study are available from the corresponding author on reasonable request. 


\section{Acknowledgements}

We thank André Gidding, Léon Westerd, Joop Woelke and Frans van Aggelen for culturing insects and Unifarm of Wageningen University for providing plants for the field experiments. We thank Erik Poelman for the self-fertilised B. nigra lines, help with setting up the field experiments and fruitful discussions. Furthermore, we thank Emilie Scheggetman, Michele Alfano and Ingeborg van Es, for help with some experiments. We thank the DAAD (57044990), The Netherlands Organization for Scientific Research (NWO/TTW Vidi grant 14854) and the German Research Foundation (CRC 973; www.sfb973.de) for funding.

\section{Author Contributions}

All authors conceived and designed the experiments. E.G. and N.E.F. performed experiments, conducted fieldwork. E.G. conducted statistical analysis and wrote the first draft of the paper. All authors interpreted results,drafted and revised the article. 


\section{A Supplementary material}

Abiotic factors None of the other tested abiotic factors affected egg survival, namely relative humidity (GLM, $\chi^{2}=0.39$, df $=1, P=0.53, \chi^{2}=0.14$, df $=1, P=0.71, \chi^{2}=$ $1.92, \mathrm{df}=1, P=0.17)$, mean daily temperature $\left(\mathrm{GLM}, \chi^{2}=0.57, \mathrm{df}=1, P=0.45, \chi^{2}=\right.$ $0.17, \mathrm{df}=1, P=0.68, \chi^{2}=0.35, \mathrm{df}=1, P=0.56$ ) and mean total daily radiation (GLM, $\left.\chi^{2}=3.05, \mathrm{df}=1, P=0.08, \chi^{2}=0.16, \mathrm{df}=1, P=0.69, \chi^{2}=2.24, \mathrm{df}=1, P=0.13\right)$. 


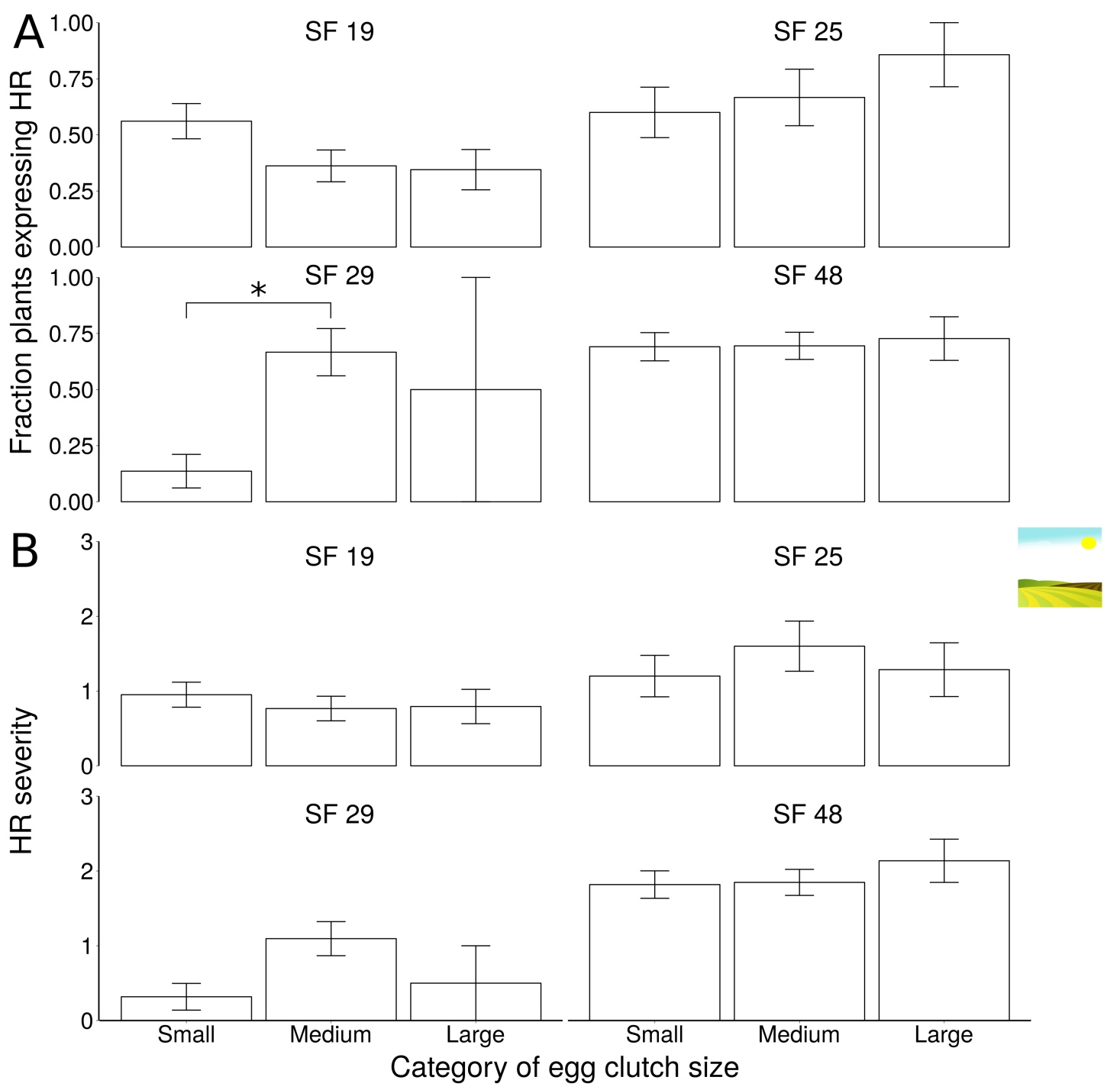

Figure 2.A.1: Relationship between egg clutch size (in categories, Small: 6-50 eggs, Medium: 51-100 eggs, and Large: more than 100 eggs) and (A) the fraction of plants expressing HR, and (B) the fraction of plants showing a particular HR severity, as recorded in field experiments. All four plant accessions are separately depicted, and only significant differences are shown to keep the graph simple (*: $P<0.05)$. 


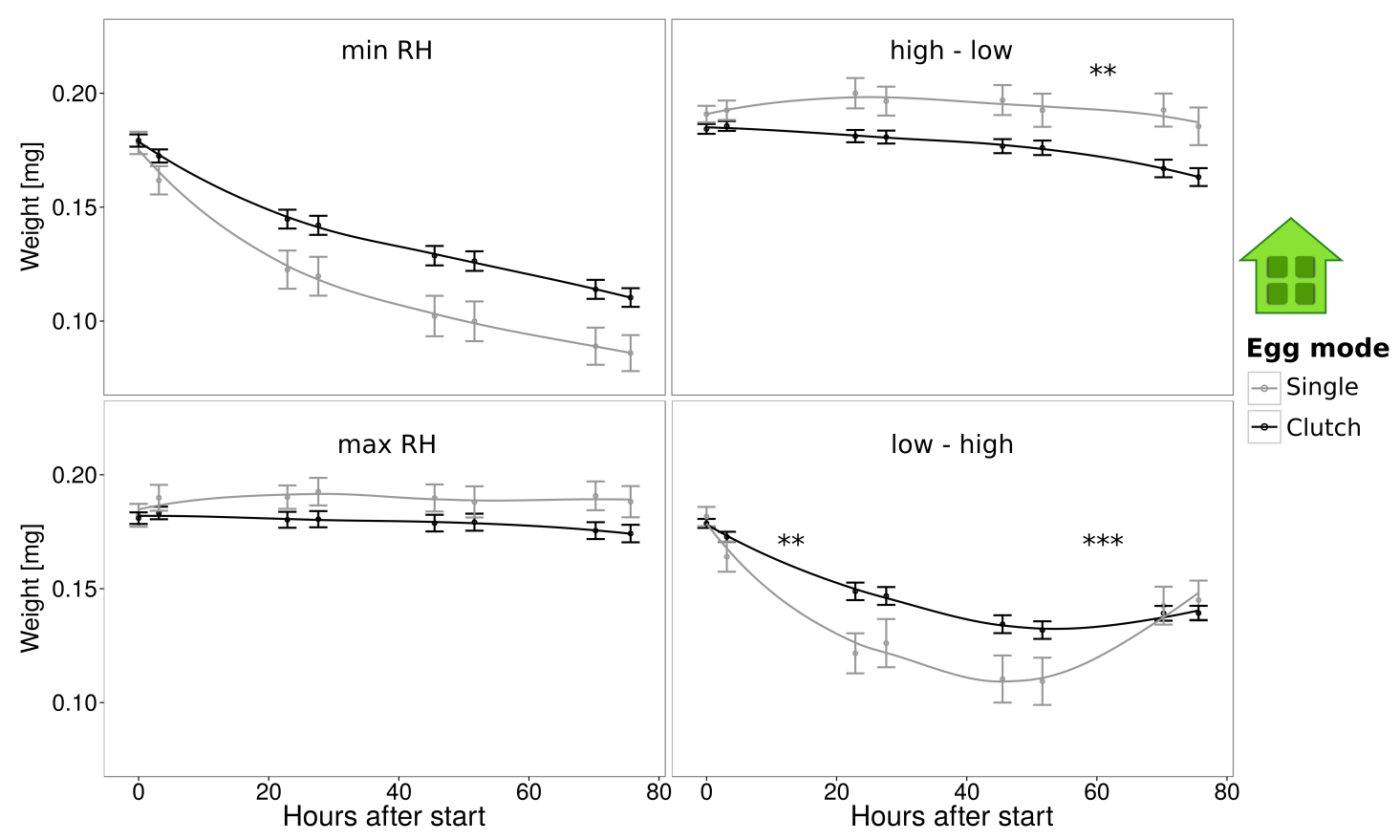

Figure 2.A.2: Weight gain or loss of single eggs or egg clutches exposed to various humidity conditions (compare Fig. 2.A.6 for humidity conditions). Data points are shown as means ( \pm SE). Asterisks between two measuring points indicate significant differences in weight change between single and clustered eggs. $* *: P<0.01, * * *: P<0.001$. For greater clarity only significant results are marked. 

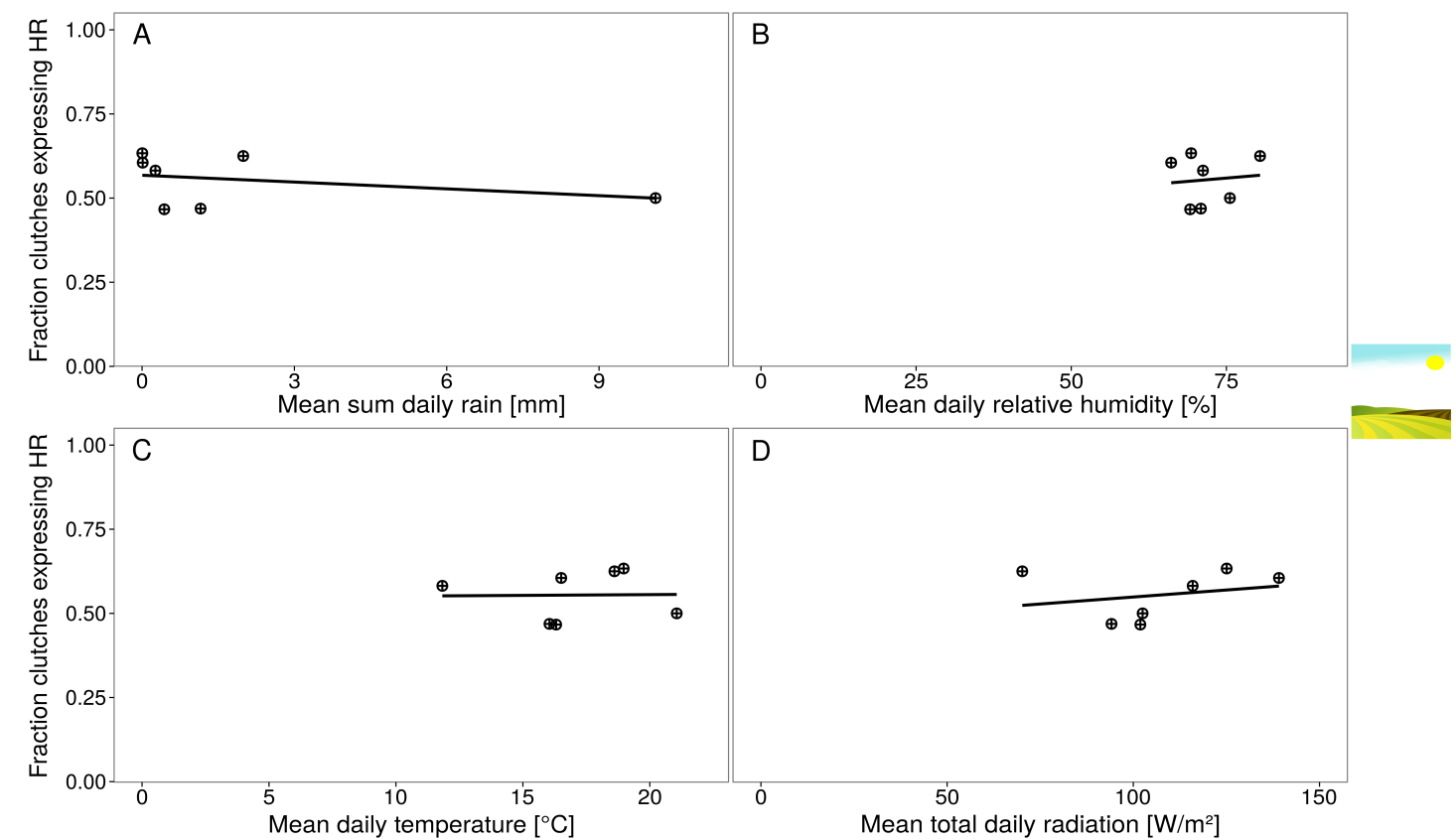

Figure 2.A.3: Relationship between the fraction of egg clutches inducing HR in the field and abiotic conditions during 2013 and 2014. (A) Mean sum of daily rainfall. (B) Mean daily relative humidity. (C) Mean daily temperature. D) Mean daily total radiation.

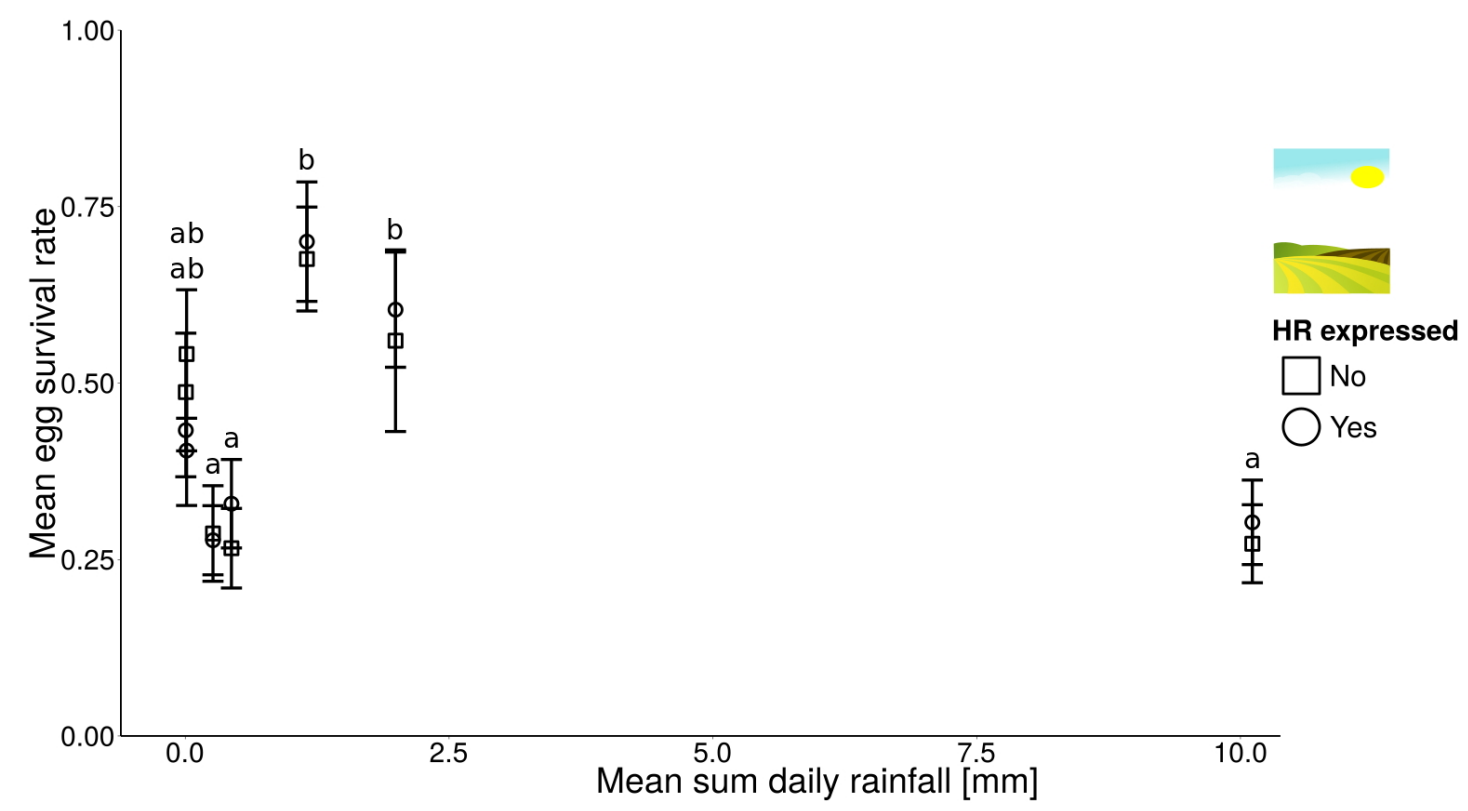

Figure 2.A.4: Egg survival rate in the field under different rainfall conditions. The sum of daily rainfall did significantly influence egg survival under field conditions (different letters indicate $P<$ 0.05 , pairwise MWU test). 


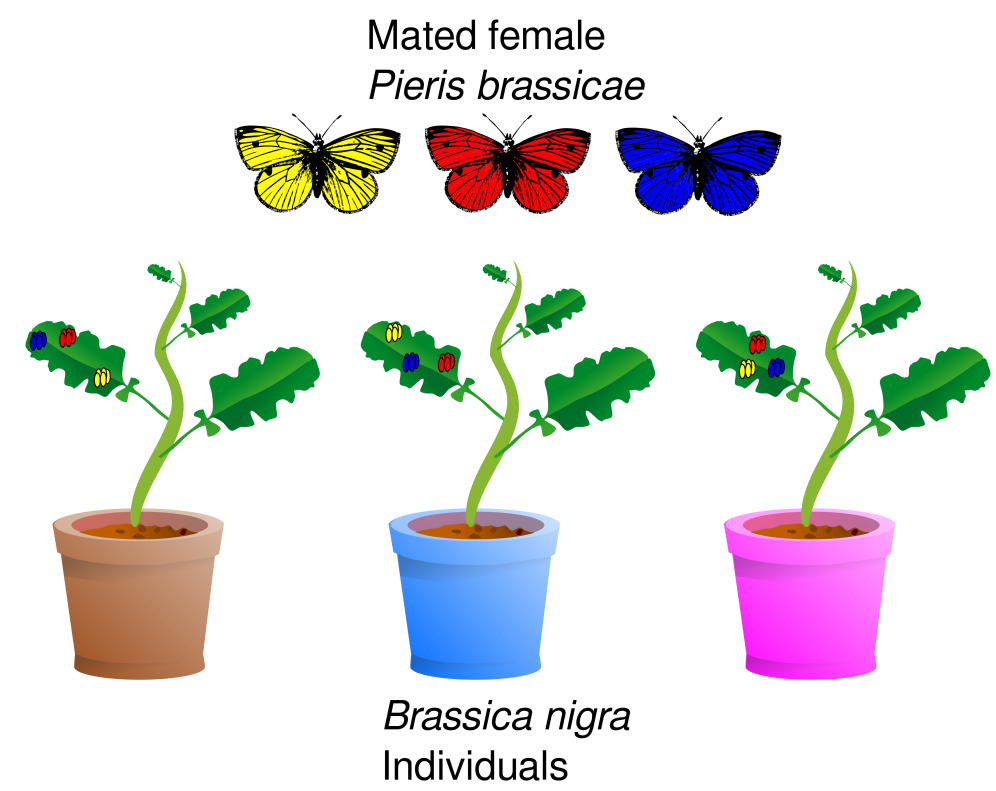

Figure 2.A.5: Schematic outline of the experiment on the impact of a plant individual on formation of HR-like necrosis. Each butterfly individual was allowed to lay a clutch of five eggs (eggs of the same colour originate from the same butterfly) per plant (represented through differently coloured pots). While only three butterflies and plants are shown here, for the experiment eight to ten plants and butterflies were used.
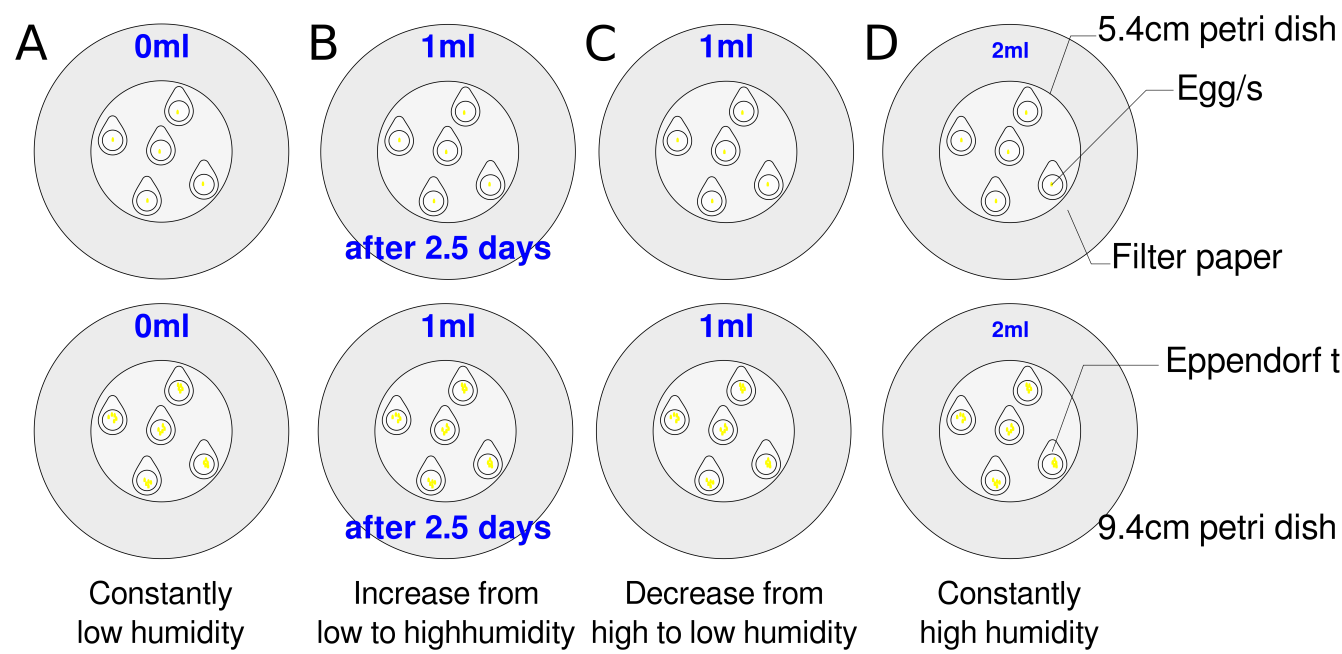

Increase from

Decrease from low to highhumidity high to low humidity

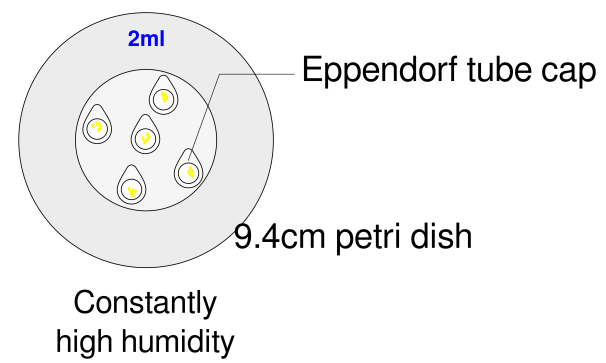

Figure 2.A.6: Experimental setup for investigating the impact of various humidity conditions on egg survival. Eggs were placed into Eppendorf caps for easy weighing and also protecting them from direct contact with the water on the filter paper. (A) $0 \mathrm{ml}$ water was added to the filter paper and the dish was kept closed for 4.5 days ("min RH"). (B) $1 \mathrm{ml}$ was added to the filter paper, but only 2.5 days after placing the eggs in the dish; the dish was kept closed ("low $\rightarrow$ high RH"). (C) $1 \mathrm{ml}$ water was added, slow evaporation over time reduced the water content to $0 \mathrm{ml}$ ("high $\rightarrow$ low RH"). (D) $2 \mathrm{ml}$ water were added; eggs were kept at humid conditions for 4.5 days. Larval hatching rates were recorded to determine egg survival rates. 


\section{Chapter 3}

Effects of oviposition by Pieris brassicae and plant accession on plant volatiles and behaviour of three insect species

Eddie Griese, Marcel Dicke, Erik H. Poelman, Berhane T. Weldegergis, M. Eric Schranz, Monika Hilker \& Nina E. Fatouros

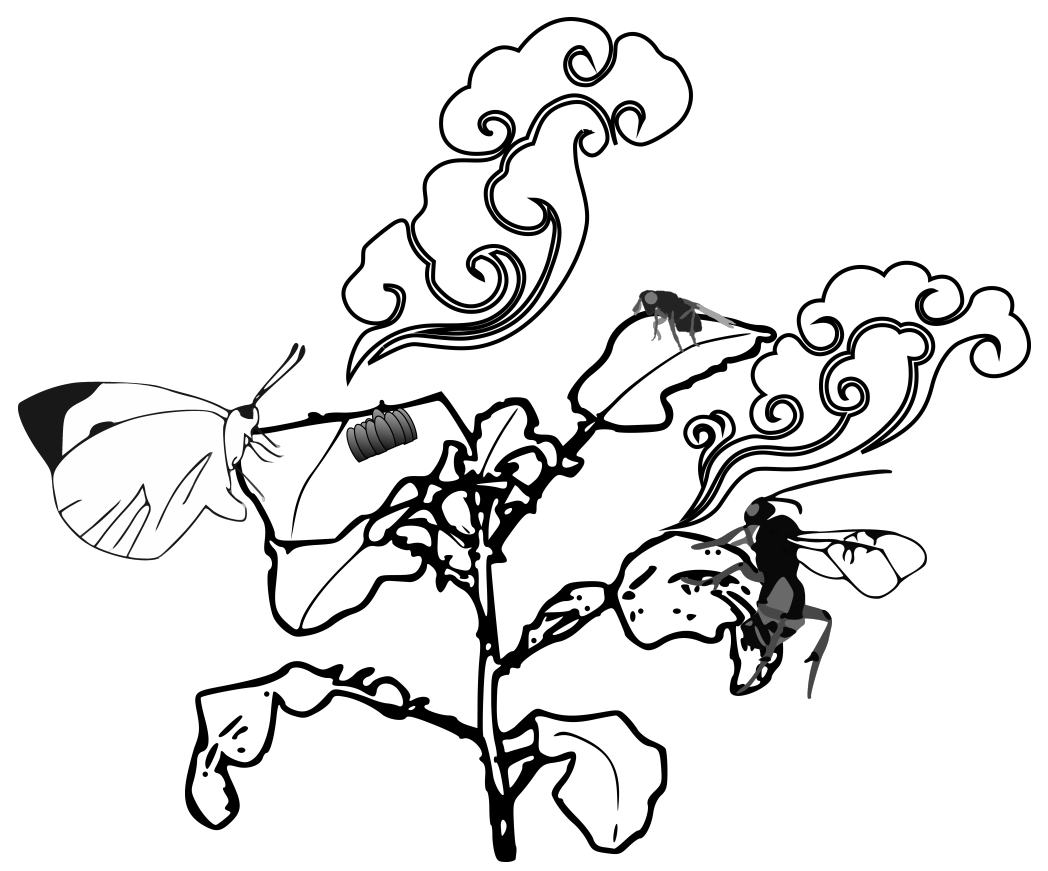




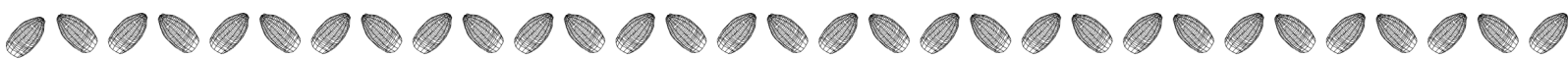

\section{Abstract}

Plants can respond to insect oviposition with the emission of volatiles, which mediate interactions among plants, herbivores and natural enemies of herbivorous insects. Little is known about plant intraspecific variation of such oviposition-induced plant volatiles (OIPVs) and how such variation affects trophic interactions. We addressed these gaps in knowledge by investigating interactions between black mustard Brassica nigra, the butterfly Pieris brassicae, and its egg and larval parasitoids. We used two B. nigra accessions (SF19 and SF48). Our GC-MS analyses revealed that the two B. nigra accessions constitutively emitted the same volatile compounds, but when induced by egg deposition, they showed quantitative differences in the emission of the homoterpene (E)-4,8-dimethyl-1,3,7-nonatriene ((E)-DMNT) and the sequiterpene $\alpha$-funebrene. Our laboratory studies revealed that the insects' behavioural responses to egg-laden and eggfree plants differed. While $P$. brassicae avoided egg-induced B. nigra plants for further egg deposition, the egg parasioid Trichogramma evanescens was attracted to them. These differences were independent of the plant accessions. In the field, P. brassicae larvae feeding upon SF48 plants suffered higher parasitism rates by the larval parasitoid Cotesia glomerata than larvae on SF19 plants. However, this effect was independent of prior egg induction of the plants. Our results suggest that intraspecific differences in plant responses to insect eggs exist, and that this variation affects insect behaviour depending on the insect species.

\section{Introduction}

Plants emit a huge variety of volatile organic compounds that play important roles in their interaction with the biotic environment, such as herbivores, natural enemies, pollinators, neighbouring plants and microorganisms (Bouwmeester et al., 2019). Such volatiles are emitted constitutively or induced by herbivore feeding and/or egg deposition (Loreto et al., 2014). The specific blends released differ due to a wide range of factors such as the abiotic and biotic environment or internal differences such as plant genotype and age (Bouwmeester et al., 2019; Dicke and Baldwin, 2010; Erb, 2018). 
To protect themselves from feeding herbivores, plants can release specific herbivoreinduced plant volatiles (HIPVs) (Dicke and Baldwin, 2010; Poelman et al., 2009b; Price et al., 1980; Turlings and Erb, 2018), which are attractive to parasitoids and predators of the herbivores (Heil, 2004; Poelman et al., 2009b; Xiu et al., 2019). Plant responses to insect oviposition can already protect against an initial developmental stage of herbivorous insects, i.e. the eggs. Oviposition by insect herbivores has been shown to induce volatiles (OIPVs) that attract egg and larval parasitoids and deter herbivores from oviposition (Fatouros et al., 2012; Hilker and Fatouros, 2015; Hilker and Meiners, 2010; Hilker and Meiners, 2006; Tamiru et al., 2011). The feeding- and egg-induced volatile blends of different plant species or plants infested by different insect species differ and cause differential attraction of larval or egg parasitoids (Baldwin, 2010; Dudareva et al., 2006; Turlings and Erb, 2018; Meiners et al., 2003).

Detecting plant genotypes with OIPV and HIPV emissions highly attractive to natural enemies of the herbivore and non-attractive or even repellent to the herbivores might be helpful for biological control of herbivorous insects infesting crop plants (Tamiru et al., 2017). The so-called "push-pull” biocontrol strategy makes use of differences in plant attractiveness by pushing herbivorous insects away from crop plant species and pulling them into fields with attractive "trap" plant species (Cook et al., 2007). Plant accessions, which differ in leaf odour, could also be used in this context by growing "push" accessions with odour repelling herbivorous insects and "pull" accessions with attractive odour to natural enemies of the herbivores. Yet, further research on both OIPVs and HIPVs at natural conditions and different spatial scales is needed to elucidate the ecological effects of these volatiles in trophic webs. Information on this is crucial for our understanding of tritrophic interactions and possible application in agroecosystems (Aartsma et al., 2017).

Here, we investigated whether herbivorous and parasitic insects discriminate between different accessions of a plant species known to release OIPVs and HIPVs in response to insect oviposition and feeding, respectively. Previous studies of tritrophic interactions between Brassica nigra, Pieris spp., and egg and larval parasitoids provide an excellent basis to pursue this aim. Black mustard plants (B. nigra) are known to respond to Pieris spp. egg deposition with the emission of volatiles that attract egg parasitoids (Trichogramma spp.) and larval parasitoids (Cotesia glomerata) under laboratory conditions (Cusumano et al., 2015; Fatouros et al., 2012; Fatouros et al., 2014; Pashalidou 
et al., 2015c; Ponzio et al., 2016). Gravid P. brassicae females were shown to be deterred by OIPVs released by the same plants (Fatouros et al., 2012). Plant responses to $P$. brassicae egg deposition can also affect subsequent responses to hatching larvae. Previously egg-laden $B$. nigra plants release volatiles induced by feeding activity of neonate larvae earlier than egg-free plants; the feeding-induced HIPVs attract $C$. glomerata wasps (Pashalidou et al., 2015c).

While intraspecific variation of B. nigra OIPVs induced by Pieris oviposition has not been studied so far, such variation has been shown for another egg-induced resistance trait: Pieris eggs induce a hypersensitive response (HR)-like leaf necrosis. Some B. nigra accessions express HR-like symptoms in response to egg deposition by $P$. brassicae more frequently than others (Griese et al., 2017). HR-like necrosis can lead to desiccation and thus death of singly laid Pieris eggs (Fatouros et al., 2014; Griese et al., 2017). Interestingly, B. nigra plants can synergistically express both resistance traits in response to Pieris egg deposition. Volatiles of plants expressing HR-like symptoms in response to Pieris eggs were more attractive to Trichogramma egg parasitoids, than volatiles of non-HR expressing plants. This synergistic effect of different egg-killing traits seems more common in Brassica species (Afentoulis et al., 2021). In the field, this 'double-defence line' was shown to lead to up to $80 \%$ egg mortality on $\mathrm{B}$. nigra plants expressing HR-like response (Fatouros et al., 2014). These results suggest that the blend of OIPVs emitted by B. nigra varies between accessions and that HR-like response could play a role in here.

Here, we analysed whether two accessions of B. nigra (SF48 and SF19) differ in the emission of OIPVs induced by P. brassicae oviposition. Previously, SF48 was shown to express a stronger HR-like response both in frequency and severity than SF19 (Griese et al., 2017). Under greenhouse conditions, we further studied whether gravid P. brassicae butterflies and T. evanescens egg parasitoids show differential behavioural responses to the two accessions. Additionally, we investigated in a semi-field set-up whether parasitism rates of larvae feeding upon the two plant accessions differ from each other and whether plant responses to egg deposition play a role in here (Figure 3.1). Parasitism rates observed in field/semi field studies were previously shown to be reliable predictors of attraction of Cotesia parasitoids to HIPVs in the laboratory (Poelman et al., 2009b). We hypothesize that SF48 shows quantitative differences in the emission of OIPVs when compared to SF19. Furthermore, we expect that the responses of $P$. brassicae as well as 
of egg- and larval parasitoids to egg-induced plants differ between the accessions.

Specifically, we addressed five questions. (i) Does $P$. brassicae egg deposition induce different OIPV blends in two different $B$. nigra accessions? (ii) Does egg deposition by conspecifics affect $P$. brassicae butterfly oviposition preferences to different $B$. nigra plant accessions? (iii) Does the emission of OIPVs differentially affect attraction of Trichogramma egg parasitoids to different $B$. nigra accessions? (iv) Do plant responses to egg deposition differentially affect parasitism rates of Trichogramma spp. and Cotesia glomerata on different B. nigra accessions? (v) Do plant responses to egg deposition differentially affect fitness in different $B$. nigra accessions?

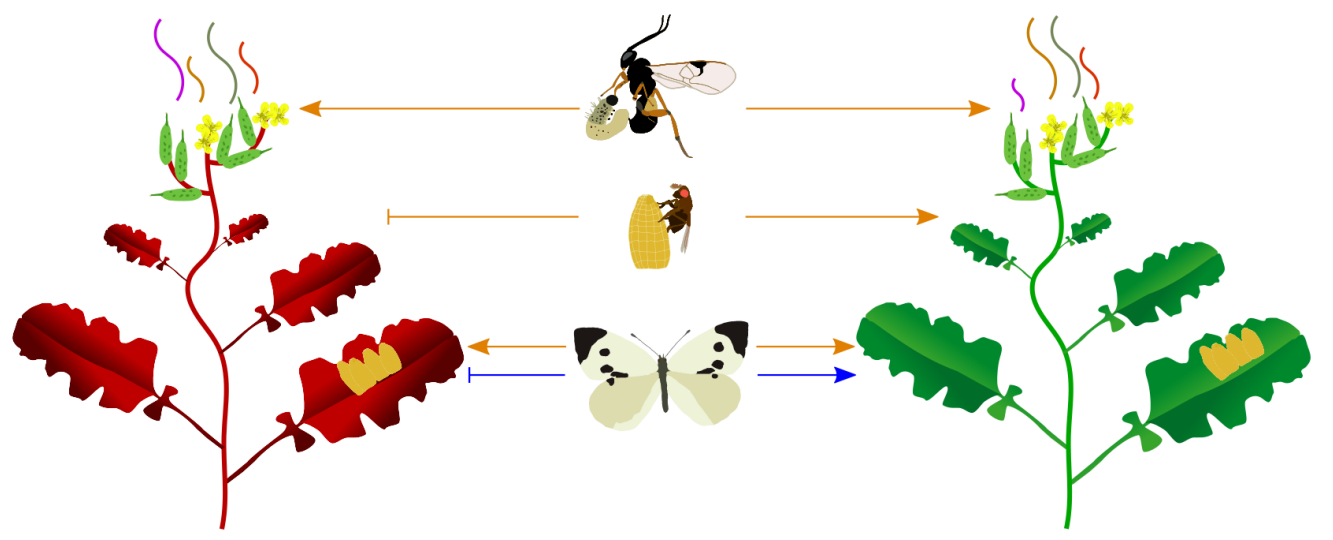

Figure 3.1: Possible scenarios outlining how insects might respond to volatile emissions of different accessions of a plant species with and without insect egg depositions. Two different plant accessions might either attract (arrows) or repel ( $\mathrm{T}$ intersection) gravid butterfly females, egg and larval parasitoids. Different responses of insects to uninfested plants (blue lines) and larval/egg-infested plants (orange lines) may be observed. For example, the right accession is attractive to all insects, irrespective of the presence of eggs on the leaves. In contrast, the left accession without eggs is neither attractive to the butterfly nor to the egg parasitoid. The volatile bouquet of the leaves is represented by wavy lines. Size and colour of the lines represent different compounds within a blend, which are expected to quantitatively differ between accessions and/or egg-induced vs. control plants.

\section{Results}

Variation in oviposition-induced plant volatiles Chemical analysis of shoot volatiles released by the two B. nigra accessions (SF19 and SF48) treated with P. brassicae eggs or left untreated revealed that all plants emitted the same compounds, but in different quantities. In total, 44 volatile compounds were detected in more than $50 \%$ of all plant 
treatments/accessions (Figure 3.2, Table 3.A.1). Overall, the entire blends of volatiles released by the differently treated accessions could not be separated by a cluster analysis (Figure 3.2). Moreover, when comparing the effect of egg treatment, plant accession and the interaction between these factors using a two-way ANOVA or GLM for each volatile compound, the interaction between egg treatment and plant accession was never significant.

However, we found that the main effect of egg treatment significantly affected quantities of five compounds, namely: the alcohol 1-penten-3-ol, the ester hexyl acetate and three terpenoids, specifically the monoterpene linalool, the sesquiterpene $\beta$-caryophyllene, and the homoterpene (E,E)-4,8,12-trimethyltrideca-1,3,7,11-tetraene ((E,E)-TMTT) (Figure 3.2). While quantities of 1-penten-3-ol, hexyl acetate and (E,E)-TMTT were lower in the headspace of plants with eggs, the emission of linalool and $\beta$-caryophyllene was induced by egg deposition (Table 3.A.1). Post-hoc tests revealed that linalool quantities significantly differed between egg-free and egg-induced plants for both accessions. Quantities of $\beta$-caryophyllene were only significantly higher in egg-treated SF48 plants than in egg-free SF48 plants; such a difference was not detectable for egg-free versus egg-treated SF19 plants (Figure 3.2).

Furthermore, quantities of two more terpenoids, the homoterpene (E)-4,8-dimethyl1,3,7-nonatriene ((E)-DMNT) and the sesquiterpene $\alpha$-funebrene, significantly differed in dependence of the accession, but were not affected by egg treatment (Figure 3.2). While quantities of $\alpha$-funebrene only differed significantly between both accessions after egg deposition, quantities of (E)-DMNT differed between the accessions only for egg-free plants (Figure 3.2). For detailed information on peak areas, see Table 3.A.1.

Thus, while neither accession nor egg infestation affected the entire blend composition of the volatiles emitted, we found differences in quantities of several single compounds. Egg deposition by P. brassicae seems not to change the total volatile emission of $B$. nigra plants, which is in accordance with previous findings for the same system (Fatouros et al., 2012; Ponzio et al., 2016).

Behavioural choice tests Butterfly oviposition preferences. Pieris brassicae females preferred to lay eggs onto control plants over egg-induced plants (GLM: $\chi^{2}=6.39$, df $=1, P=0.012$; Figure 3.3A). Plant accession did not significantly affect this behaviour (GLM: $\chi^{2}=0.39, \mathrm{df}=1, P=0.532$ ). 

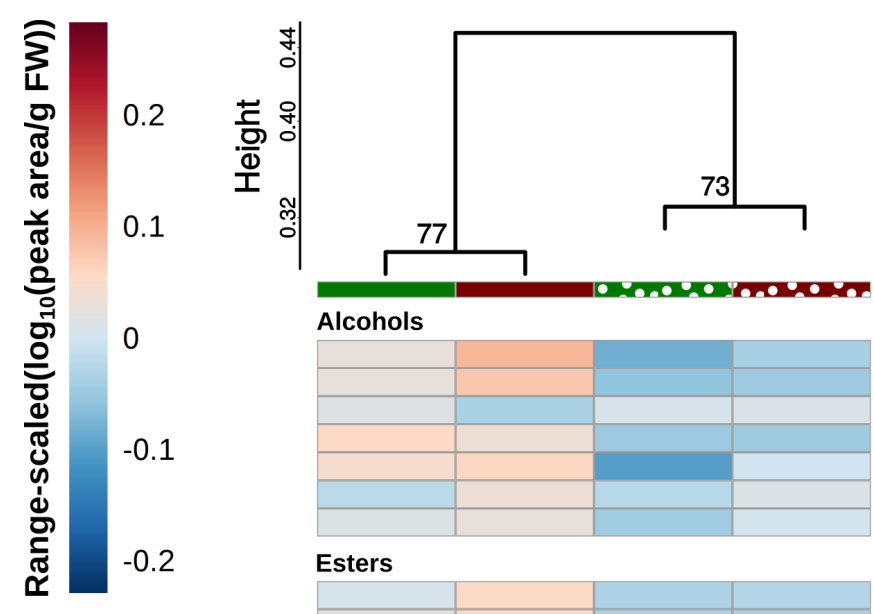

\section{Treatment}

SF19 control
SF48 control
SF19 eggs
SF48 eggs

ANOVA/GLM

E A

Alcohols

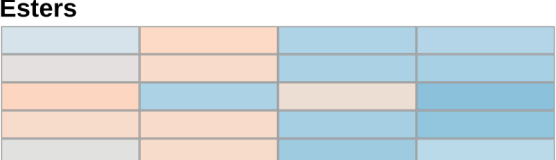

Carbonyls

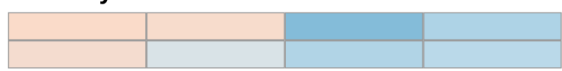

Terpenoids
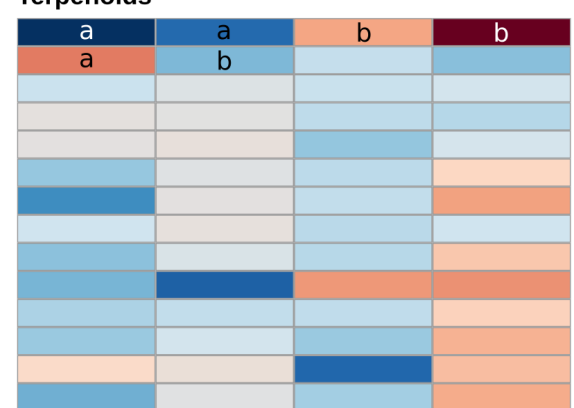

a

b
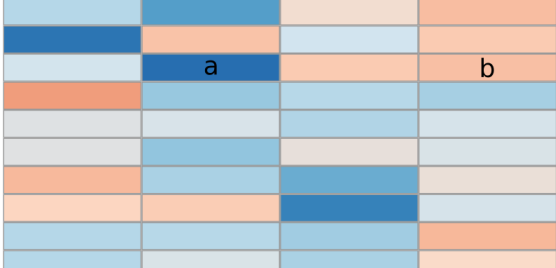

Nitrogen and/or Sulfur containing

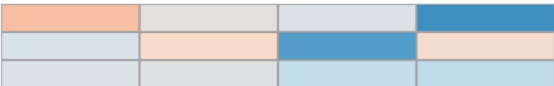

Others

Figure 3.2: Caption on next page.
(Z)-3-Hexen-1-ol

Unknown 1

6-Methyl-1-octano

1-Nonanol

1-Decanol

1-Dodecano

(Z)-3-Hexen-1-yl, acetate Hexyl acetate

Methyl salicylate

Methyl decanoate

Methyl dodecanoate

Undecanal

6,10-Dimethyl-2-undecanone

Linalool

(E)-DMNT

Menthol

cis-Verbone

cis-2,3-Pinanediol

7- $\alpha-\mathrm{H}$-Silphiperfol-5-ene

Presilphiperfol-7-ene

Tetrahydroionyl acetate

7-epi-Silphiperfol-5-ene

a-Cubebene

Silphiperfola-5,7(14)-diene

7- $\beta$ - $\mathrm{H}$-Silphiperfol-5-ene

African-1-ene

Silphiperfol-6-ene

a-Funebrene

$\beta$-Cubebene

a-Cedrene

$\beta$-Caryophyllene

(E)- $\beta$-Farnesene

$\alpha$-Isomethyl ionone

$\delta$-Cadinene

Neryl isovalerate

$(E, E)$-TMTT

TUTM

IDMOM

*

Benzothiazole

Unknown 2

Anisole

1,3,3-Trimethyl-1-phenylindan 
Figure 3.2: (Previous page.) Dendrogram and heatmap of the quantitative emission of volatile compounds of two different Brassica nigra accessions (SF48 and SF19) infested with Pieris brassicae eggs or uninfested plants. For the heat map, we used range-scaled log10-transformed values of volatile emission (peak area/plant weight $[\mathrm{g}] \times 10^{-5}$ ) for each compound. Ward's clustering algorithm with Euclidean distances was used for performing dendrogram clustering. Next to the nodes on the cluster an AU $P$-value is displayed, a value greater than 95 would indicate significant differences. Boxes with small letters indicate significant differences between $B$. nigra accession and differently treated accessions (eggs/no eggs) (t-test or MWU test $P<0.025$, Bonferroni corrected). Only significant differences are labelled by different letters. Asterisks indicate significant differences of the main factors (*: $P$-value $<0.05$, ***: P-value $<0.001$ ), egg treatment (E) and plant accession (A), tested by two-way ANOVA or GLM are shown in a table on the right side of the heat map. The interaction term was never significant and is therefore not shown. The abbreviated compounds are: (E)-DMNT: (E)-4,8-dimethyl-1,3,7-nonatriene, (E,E)-TMTT: (E,E)-4,8,12-trimethyltrideca-1,3,7,11-tetraene, TUTM: tricyclo[6.3.0.0(1,5)]undec-2-en-4-one, 2,3,5,9,tetramethyl, and IDMOM: (7a-isopropenyl-4,5-dimethyloctahydro-inden-4-yl)methanol.

When comparing untreated plants of the two accessions directly, the butterflies did not discriminate $(\mathrm{t}=0.841, \mathrm{n}=10, P=0.422$, Figure 3.A.1). The same was true when egg-induced plants were compared $(\mathrm{t}=0.617, \mathrm{n}=10, P=0.551$, Figure 3.A.1). Thus, ovipositing females clearly discriminate between control and egg-laden plants, but do not discriminate between the two accessions.

Responses by egg parasitoids to OIPVs. Trichogramma egg parasitoids significantly preferred egg-induced plants over control plants (GLM: $\left.\chi^{2}=9.66 \mathrm{df}=1, P=0.002\right)$. However, plant accession, wasp line, and their interaction did not influence the attraction significantly (GLM: $\chi^{2}=1.18 \mathrm{df}=1, P=0.277 ; \chi^{2}=0.281 \mathrm{df}=2, P=0.869 ; \chi^{2}=$ $3.525 \mathrm{df}=2, P=0.172$, respectively, Figure 3.3B). When checking for each wasp line and plant accession pair separately none of the combinations showed a significantly higher attraction $(E d 16 / S F 19: \mathrm{t}=-0.16, \mathrm{n}=7, P=0.878$; PB1-III/SF19: $\mathrm{t}=0.167, \mathrm{n}$ $=7, P=0.873 ;$ PB1-III/SF48: $\mathrm{t}=1.132, \mathrm{n}=7, P=0.301 ;$ PB2-II/SF19: $\mathrm{t}=2.198, \mathrm{n}$ $=7, P=0.070$; PB2-II/SF48: $\mathrm{t}=0.459, \mathrm{n}=7, P=0.663$, Figure 3.A.2). Wasps of the isofemale line Ed16 almost significantly preferred OIPVs emitted by SF48 plants above control SF48 plants $(\mathrm{t}=2.356, \mathrm{n}=7, P=0.057)$.

Larval parasitism rates in the field We investigated whether larval parasitism rates differ in dependence of plant accession and previous egg treatment. Plant accession had a significant effect, the fraction of parasitized caterpillars was significantly higher 


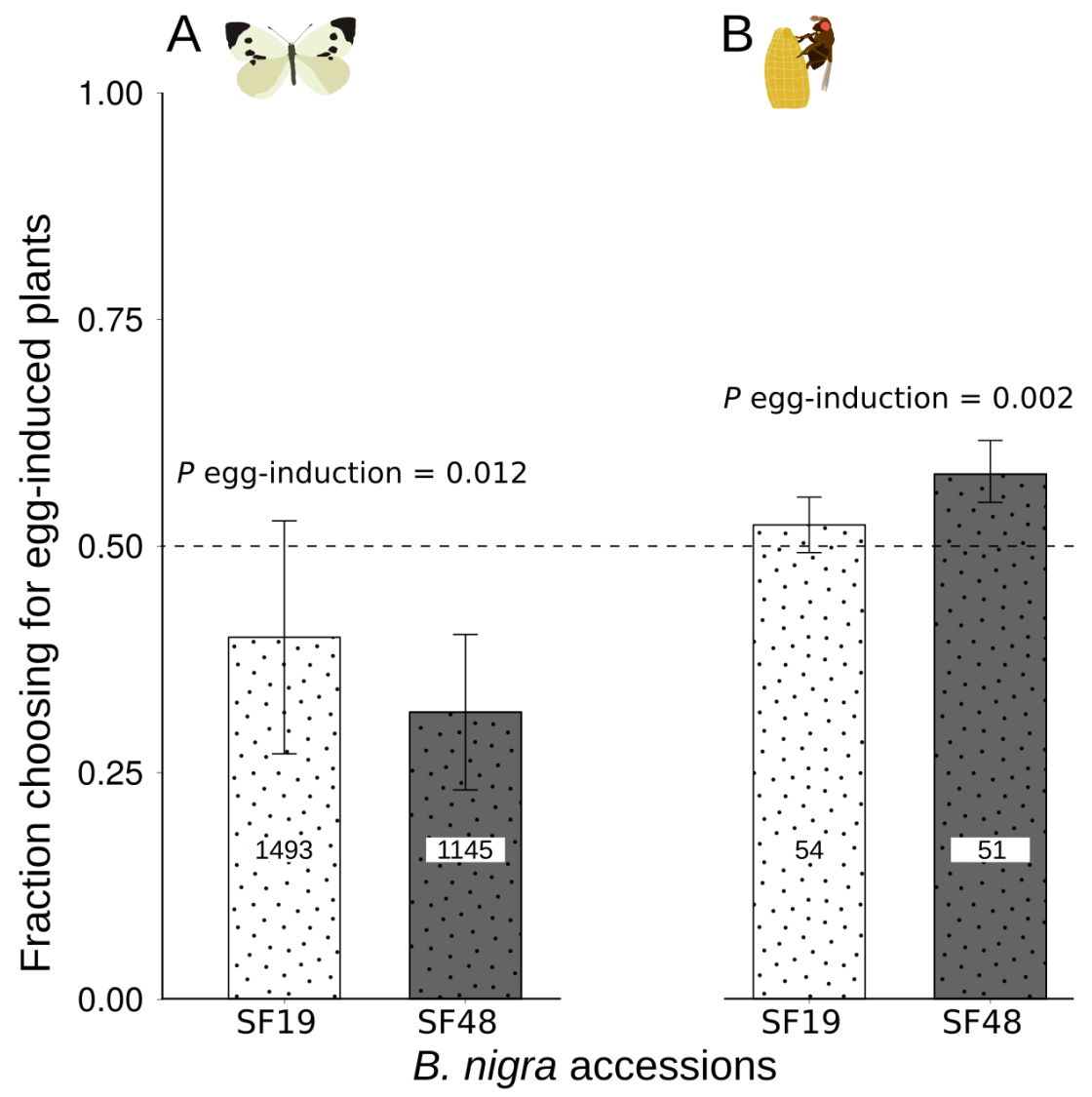

Figure 3.3: Behavioural responses to egg-infested B. nigra plants (accession SF19 or SF48) of gravid $P$. brassicae butterflies and T. evanescens females. (A) Mean ( \pm SE) fraction of $P$. brassicae eggs laid on egg-infested plants when compared to control plants of the same accession. The number of eggs laid onto previously egg-laden plants within $24 \mathrm{~h}$ was counted and divided by all eggs laid. Number of plant pairs tested per accession, each with a different butterfly, $n=12$. (B) Mean ( $\pm \mathrm{SE}$ ) fraction of T. evanescens wasps choosing odour of egg-infested plants in a Y-tube olfactometer. Proportion of wasps choosing volatiles of egg-infested plants when compared to control plants of the same accession is shown. Number of plant pairs tested per accession, each with a different set of parasitoids, $n=7$. Plants were tested $24 \mathrm{~h}$ after egg deposition. The total number of eggs laid by females, or wasps choosing in the experiments are given in the bars. 
on SF48 $(0.86 \pm 0.06)$ than on SF19 plants $(0.57 \pm 0.08)\left(\right.$ GLMM: $\chi^{2}=26.12$, df $=1, P<0.001)$. Egg treatment did not significantly change the parasitism rate when considering this for both accessions, egg-free $(F)(0.66 \pm 0.08)$ and egg-induced plants (EF) $(0.78 \pm 0.06)\left(\mathrm{GLMM}: \chi^{2}=3.27, \mathrm{df}=1, P=0.07\right)$. However, the interaction between accession and egg infestation was significant (GLMM: $\chi^{2}=12.08, \mathrm{df}=1, P$ $<0.001)$. A significantly smaller fraction of caterpillars was parasitized when feeding on egg-free SF19 $(0.41 \pm 0.11)$ than on egg-infested SF19 (0.72 \pm 0.10$)$ plants (EMM: $\mathrm{Z}=3.30, P=0.005$, Figure 3.4A). No such difference was observed for SF48 plants (EMM: $\mathrm{Z}=-1.13, P=0.67$, Figure 3.4A).

Prior $P$. brassicae egg deposition on plants influenced the number of $C$. glomerata larvae or eggs found per $P$. brassicae caterpillar. The main effect of egg treatment was significant, indicating that more $C$. glomerata were found in caterpillars feeding on EF plants $(14.38 \pm 0.67)$ than in caterpillars feeding on $\mathrm{F}(12.19 \pm 0.44)$ plants (GLMM: $\chi^{2}=78.84$, df $=1, P<0.001$, Figure 3.4B). Numbers of $C$. glomerata found per $P$. brassicae caterpillar did not differ between the two plant accessions. The interaction between the two factors "accession" and "egg treatment" was not significant (GLMM: $\chi^{2}=2.75, \mathrm{df}=1, P=0.10$, and $\chi^{2}=0.20, \mathrm{df}=1, P=0.66$, respectively, Figure 3.4B).

Field experiment on egg parasitism In an additional common-garden set-up, we tested whether egg parasitism differed between plant accessions and whether higher egg mortalities lead to higher plant fitness. Overall, a low number of $P$. brassicae clutches were parasitized by Trichogramma egg parasitoids (one clutch was deposited per plant), and the percentage of parasitized egg clutches was the same on SF48 plants as on SF19 (31\% for SF48 vs. $21 \%$ for SF19, $P=0.35$, Supplementary information and Supplementary Figure 3.A.4A). Moreover, we previously showed that HR-like necrosis induced by P. brassicae eggs was significantly stronger on SF48 (ca. 70\%) than on SF19 (ca. 43\%) plants. However, mortality of clustered eggs was not affected and independent of egg density (Griese et al., 2017). Finally, as egg mortalities due to parasitism and HR-like necrosis did not differ between accessions, we did not find any indication that plant fitness proxies would be affected (Supplementary Figure 3.A.5). Thus, more field studies need to be conducted to test the adaptiveness of such indirect plant resistance traits. 
A
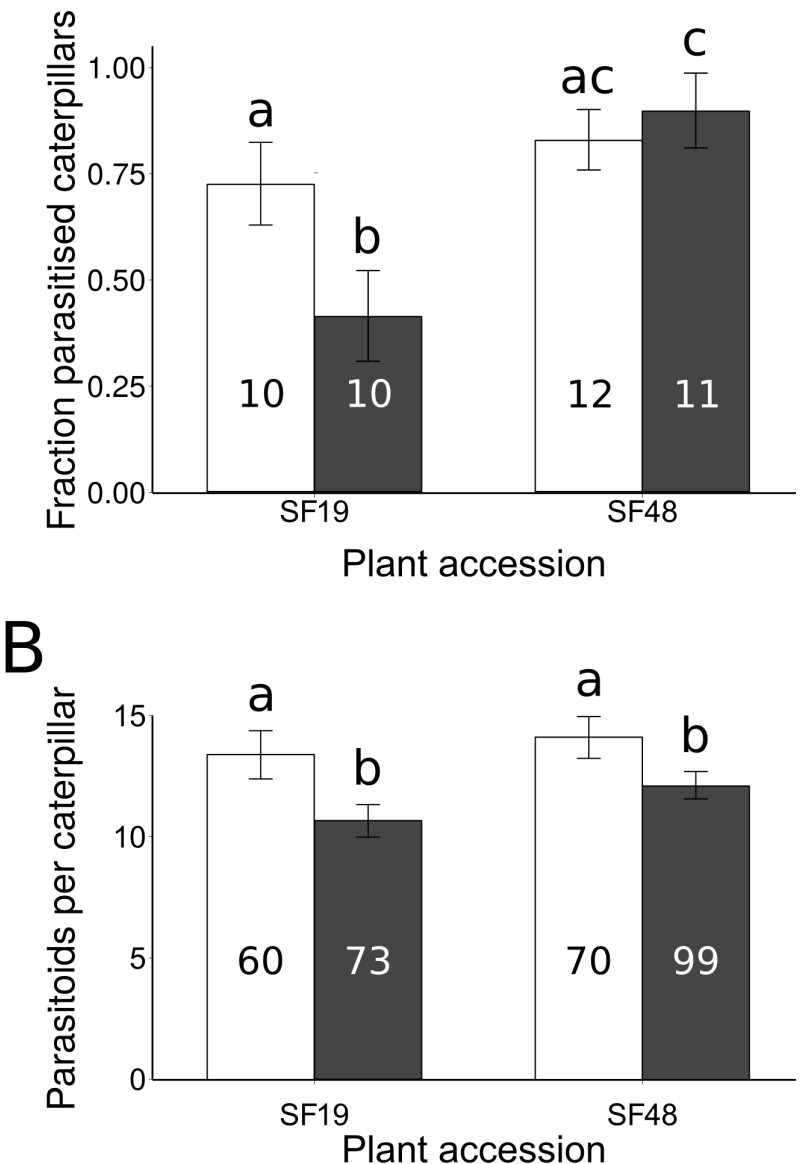

Figure 3.4: Cotesia glomerata parasitism rates of $P$. brassicae caterpillars feeding on different $B$. nigra accessions (SF48, SF19) in a semi-field experiment. Plants were either previously infested with $P$. brassicae eggs $(\mathrm{EF})$ or egg-free $(\mathrm{F})$. (A) weighted mean $( \pm \mathrm{SE})$ fraction of parasitized caterpillars per plant. Numbers in bars represent the number of plants used per group as replicates. (B) weighted mean $( \pm \mathrm{SE})$ number of $C$. glomerata larvae and eggs per $P$. brassicae caterpillar. Numbers in bars represent the number of caterpillars for each group as replicates. Different letters mean significant differences $(P<0.05)$, GLMM/EMM. 


\section{Discussion}

Our study shows intraspecific variation among two $B$. nigra accessions in the emission rates of two plant volatiles induced by egg deposition. However, the insect behaviour can only be partly explained by differences between plant accessions and was mainly driven by the effects of egg induction. While the emission of one sesquiterpene, $\beta$ caryophyllene, was induced by $P$. brassicae egg deposition in the B. nigra accession SF48 compared to control plants, no such induction was detected in accession SF19. Butterflies avoided oviposition on previously egg-laden plants and the egg parasitoid $T$. evanescens preferred volatiles of these plants over egg-free plants. This is in accordance with a previous study conducted with a different $B$. nigra accession and a different Trichogramma species (T. brassicae) (Fatouros et al., 2012). We did not find an effect of plant accession on P. brassicae and T. evanescens behaviour in these laboratory experiments. Results of our semi-field experiment suggest that the larval parasitoid C. glomerata does discriminate between accessions infested by $P$. brassicae host caterpillars: More caterpillars were parasitised on SF48 plants compared to SF19 plants. In addition, caterpillar parasitism rates were higher on SF19 plants induced by eggs, compared with clean SF19 plants.

Although neither egg treatment nor accession did affect the total volatile blends, our study found differences related to the egg treatment in quantities of five volatile compounds (1-penten-3-ol, the monoterpene linalool, the sesquiterpenes $\beta$-caryophyllene and (E,E)-TMTT, and the ester hexyl acetate) $24 \mathrm{~h}$ after egg deposition, and two compounds differed between the two accessions ( $\alpha$-funebrene and (E)-DMNT). While quantitative changes of volatile blends have been reported to be perceived by insects before, generally the importance of changes in single compounds has been established (Beyaert and Hilker, 2014; Bruce et al., 2005). Previous studies found similar compounds to be induced in B. nigra plants by aphids or caterpillars (1-penten-3-ol, (E)-DMNT, $\alpha$ funebrene, $\beta$-caryophyllene) (Ponzio et al., 2016) or P. brassicae eggs (1-penten-3-ol and $\alpha$-funebrene) (Fatouros et al., 2012). The emission of 1-penten-3-ol was lowered upon egg deposition in our study as well as in the study by Fatouros et al. 2012. On the other hand, the sesquiterpene $\beta$-caryophyllene was induced by egg deposition. Quantities were significantly higher in SF48 plants induced by eggs, compared to clean SF48 plants, while no such difference was recorded for SF19. 
Terpenoids are highly diverse and widespread in plants. Numerous terpenoids detected in HIPV and OIPV blends have been suggested to play a role in host foraging of both carnivorous and herbivorous insects (De Moraes et al., 2001; Fatouros et al., 2012; Gershenzon and Dudareva, 2007; Tamiru et al., 2012; Tamiru et al., 2015; Tamiru et al., 2017). Several behavioural studies confirm that natural enemies respond to terpenoids, such as (E)- $\beta$-ocimene, linalool (Dicke et al., 1990; Du et al., 1998), (E)-DMNT and (E,E)-TMTT (Dicke et al., 1990; Khan et al., 1997), and (E)- $\beta$-caryophyllene (Colazza et al., 2004; Rasmann et al., 2005; Tamiru et al., 2017). A vast amount of studies have shown that emissions of HIPVs vary tremendously among plant genotypes and that each plant genotype releases its own specific blend (Turlings and Erb, 2018). Recent studies on maize have shown variation in OIPV emission between cultivars and landraces. Oviposition by the stemborer Chilo partellus induces OIPVs in certain landraces but not in the commercial hybrids (Tamiru et al., 2011; Tamiru et al., 2012; Tamiru et al., 2015). Interestingly, (E)- $\beta$-caryophyllene production after oviposition was enhanced in one maize landrace and most attractive to the larval parasitoid Cotesia sesamiae (Tamiru et al., 2017). Similarly, enhanced (E)- $\beta$-caryophyllene production in bean plants, induced by oviposition and feeding of Nezara viridula bugs, was identified as an important attractant for the egg parasitoid Trissolcus basalis (Colazza et al., 2004). In our study, $\beta$-caryophyllene was induced in higher amounts in SF48 plants compared to SF48 control plants. This difference was not present in the other accession SF19. To understand if these volatiles could play a role in eliciting different insect behavioural responses to different accessions of the same plant species, further behavioural and electrophysiological studies are needed.

The observed differences in oviposition preferences of $P$. brassicae are likely caused by differences in plant chemistry and OIPV blends as explained above. Egg deposition has been shown to induce quantitative changes in plant volatile blends, which in turn affect oviposition preferences of herbivorous insects, including butterflies and moths (Bertea et al., 2020; Hilker and Fatouros, 2015). Similar to the work on parasitoids, $\beta$-caryophyllene present in host plant odours has been shown to elicit oviposition in codling moths (Witzgall et al., 2005) and attraction of mated females of grapevine moths to grapes (Tasin et al., 2006). We previously showed in a flight chamber set-up that gravid $P$. brassicae butterflies prefer volatiles from control B. nigra plants over volatiles from plants induced by eggs of conspecifics, while they did not discriminate between 
volatiles from untreated plants and those from plants infested with cabbage moth eggs (Fatouros et al., 2012). Because eggs on plants can act as visual cues under natural conditions (Williams and Gilbert, 1981), we here removed them before offering the plants to the butterflies for oviposition. Yet, it cannot be excluded that P. brassicae butterflies use other visual cues, such as egg-induced changes in photosynthetic activity or light reflectance (Mäntylä et al., 2017; Schröder et al., 2005) or that they "taste" compounds, such as glucosinolates in the leaf surfaces to discriminate between plants (Rothschild and Schoonhoven, 1977).

In the field, larval parasitoids such as $C$. glomerata make use of HIPVs at different spatial scales and intraspecific variation in HIPVs seems to play a role in here (Aartsma et al., 2017; Aartsma et al., 2019; Poelman et al., 2009b). Although, we did not study attraction of $C$. glomerata wasps to volatiles of the different accessions under laboratory conditions, e.g. in a windtunnel set-up, we can assume from previous work that the wasps' field parasitism rates reflect their preferences to HIPVs to some extent (Poelman et al., 2009b). In the semi-field set-up, more caterpillars were parasitized by $C$. glomerata when feeding on SF48 plants than on SF19 plants. This finding suggests that HIPVs emitted by SF48 plants were more attractive to host-searching C. glomerata females than SF19 plants. Previous butterfly egg deposition on plants seem to exert no effect on the parasitoids' preference for host caterpillars on SF48. However, for SF19, more caterpillars were parasitised on egg-induced plants compared to control plants. This could be due to priming of plant defences by egg deposition. In a previous field experiment, we showed that more caterpillars feeding on plants 'primed' by eggs were parasitized by $C$. glomerata than caterpillars on egg-free plants. This preference for caterpillars on egg-laden plants was even seen in members of the fourth trophic level (Pashalidou et al., 2015b). Priming by eggs was shown to accelerate and/or increase the emission of HIPVs. Cotesia glomerata wasps were attracted to volatiles of B. nigra plants infested with eggs just before and shortly after larvae hatched, and wasp performance was positively correlated with this preference for neonate larvae (Pashalidou et al., 2015c).

The observed differences in volatile compounds between accessions and some differences in the behavioural insect responses to these accessions are promising to further investigate the genetic variability in induced responses to Pieris eggs in Brassica crops. Earlier work showed a link between egg-induced HR-like necrosis and Trichogramma 
parasitism of $P$. brassicae eggs on B. nigra (Fatouros et al., 2014), leading to the idea of a double-defence line where direct and indirect egg killing act synergistically. The B. nigra accessions studied here were previously shown to vary in their egg-induced expression of HR-like necrosis (Griese et al., 2017), but overall were not different enough to take this factor into account in the present study (Table 3.A.2). Brassica nigra is an outcrossing species and shows heterozygosity in egg-inducible HR-like necrosis (Bassetti et al., 2021). In order to understand the effect of leaf necrosis at the site of egg deposition on parasitoid-attracting volatiles, future studies need to test more accessions including those that are homozygous with respect to formation of egg-inducible HR-like necrosis.

In conclusion, we observed differential emission of some OIPVs in two B. nigra accessions. Furthermore, while behavioural responses by $P$. brassicae and T. evanescens were affected by egg induction, but not by the accession, C. glomerata discriminated between accessions. So far, our data do not show that variation in OIPVs and its effects on insect preferences affect plant fitness under field conditions. However, we show intraspecific variation between plant accessions regarding their responses to egg deposition. Furthermore, the previously observed double-defence-line consisting of direct (HR-like necrosis) and indirect (attraction of egg parasitoids) egg-killing plant responses may promise a considerable potential to improve resistances against pests in crops via selective breeding and application in integrated pest management.

\section{Material and Methods}

Plants and insects Black mustard (B. nigra L.) plants were grown in a greenhouse compartment (18 $\left.\pm 5^{\circ} \mathrm{C}, 50-70 \% \mathrm{RH}, \mathrm{LD} 16: 8\right)$. We used two self-fertilised plant accessions, which originated from a B. nigra population collected in 2009 at the edge of the River Rhine in Wageningen, The Netherlands $\left(51.96^{\circ} \mathrm{N}, 5.68^{\circ} \mathrm{E}\right)$. These accessions, SF19 and SF48, each represent offspring of a single self-pollinated plant. SF is referring to the locations where the mother plants of these accessions were found, SF = 'Steen Fabriek' (brick factory), Wageningen, The Netherlands. These two plant accessions were previously recorded to be different in the expression of HR-like necrosis induced by Pieris egg deposition (Griese et al., 2017). Egg-induced HR-like necrosis and the emission of OIPVs were shown to co-occur and enhance mortality of P. brassicae eggs 
laid on B. nigra plants (Fatouros et al., 2014). We chose these two plant accessions differing in expression of egg-induced HR-like necrosis and studied here whether they also differ in the emission of OIPVs.

Pieris brassicae L. (Lepidoptera: Pieridae) was reared on Brussels sprout plants (B. oleracea var. gemmifera cv. Cyrus) in a climate room $\left(21 \pm 1{ }^{\circ} \mathrm{C}, 50-70 \% \mathrm{RH}, \mathrm{LD}\right.$ 16:8). Newly emerged virgin female and male P. brassicae butterflies were obtained from the rearing and kept individually isolated. Two days after emergence they were brought together to allow for mating. Further two days later the mated female butterflies were used for egg deposition in the experiments. For the experiments in the laboratory, B. nigra plants were placed into a cage filled with more than one hundred butterflies to receive butterfly eggs. After an egg clutch of about 30 eggs had been laid, the plant was removed from the cage. Additional egg clutches were carefully removed from the plants with a fine brush.

Three isofemale lines (each originating from a single female) of T. evanescens Westwood (Ed 16, PB1 - III and PB2 - II) (Hymenoptera: Trichogrammatidae) were used in the laboratory experiments. All lines originated from parasitoid females, which had emerged from $P$. brassicae egg clutches collected in a B. nigra field in Wageningen and the surrounding areas in 2012. The wasps were reared in a climate cabinet $\left(24 \pm 1^{\circ} \mathrm{C}\right.$, 50-70\% RH, LD 16:8) using Ephestia kuehniella eggs (Koppert Biological Systems, Berkel en Rodenrijs, The Netherlands) for juvenile development and honey as a food source for the adults. We used only female wasps, which were two to five days old, honey-fed and without any experience with Brassica plants and Pieris eggs.

Collection and analysis of plant volatiles We used a setup similar to that described by Ponzio et al. 2016. Plant volatiles were collected from four- and five-week-old plants. We collected volatiles from untreated and egg-laden plants of the SF19 and SF48 accessions (for each treatment and accession: 10 replicates). We refer to these accessions and treatments as follows: SF19 control, SF19 eggs, SF48 control and SF48 eggs. One day before sampling the headspace volatiles, each egg-laden plant had received $30 P$. brassicae eggs laid by a single butterfly. To collect volatiles, the pot of each plant was wrapped in aluminium foil and then placed individually into a 30-litre glass jar closed by a lid, which was attached with a metal hinge and sealed using a rubber seal. The jar was ventilated by synthetic air flowing into the jar via an inlet in the lid (flow: $315 \mathrm{ml} \mathrm{min}^{-1}$ ) 
and sucked out by an air pump via an exit tube in the lid (flow: $300 \mathrm{ml} \mathrm{min}^{-1}$ ). After leaving the glass jar, the air was pressed through stainless steel tubes containing Tenax TA (20/35 mesh; CAMSCO, Houston, TX). After sampling for three hours, the plants were removed from the glass jars. The aboveground biomass was weighed. The determined fresh weight was later related to the peak areas of specific volatiles. Control headspace samples were collected from pots filled with soil and covered by aluminium foil. Tenax-TA-filled cartridges with the trapped headspace samples were dry-purged for $15 \mathrm{~min}$ under a nitrogen flow at $50 \mathrm{ml} \mathrm{min}^{-1}$ and stored at ambient temperature until chemical analysis was performed. We followed the protocol for volatile analysis and compound identification as described in detail in Electronic Supplementary Material (ESM) 1 by Cusumano et al. 2015. We used a Thermo Trace GC Ultra gas chromatograph in combination with a Thermo Trace DSQ quadruple mass spectrometer (Thermo Fisher Scientific, Waltham, USA) for separation and analysis of plant volatiles.

The collected volatiles were thermally desorbed from the Tenax TA in an Ultra 50:50 thermal desorption unit (Markes, Llantrisant, UK) at $250{ }^{\circ} \mathrm{C}$ for 10 min under a helium flow of $20 \mathrm{ml} \mathrm{min}^{-1}$ while re-collecting the volatiles in a thermally cooled universal solvent trap at $0{ }^{\circ} \mathrm{C}$ using Unity 1 (Markes). Once the desorption process was completed, the cold trap was heated at a rate of $40^{\circ} \mathrm{Cs}^{-1}$ to $280^{\circ} \mathrm{C}$ and was kept for $10 \mathrm{~min}$ at $280^{\circ} \mathrm{C}$, while the volatiles were released to a ZB-5MSi capillary column [ $30 \mathrm{ml} \times$ $0.25 \mathrm{~mm}$ I.D. $\times 0.25 \mu \mathrm{m}$ F.T. with $5 \mathrm{~m}$ built-in guard column (Phenomenex, Torrance, CA, USA)], in splitless mode, for further separation. The GC oven was initially kept at $40{ }^{\circ} \mathrm{C}$ for $2 \mathrm{~min}$, and temperature was then raised at a rate of $6^{\circ} \mathrm{C} \mathrm{min}^{-1}$ to $280^{\circ} \mathrm{C}$ and held there for 4 min under a helium flow of $1 \mathrm{ml} \mathrm{min}^{-1}$ in constant flow mode. The DSQ MS was operated in scan mode with a mass range of 35-400 atomic mass units (amu) at 4.70 scans per second. Spectra were recorded in electron impact ionisation (EI) mode at $70 \mathrm{eV}$. The MS transfer line and ion source were set at $275^{\circ} \mathrm{C}$ and at $250{ }^{\circ} \mathrm{C}$, respectively. Compounds were tentatively identified based on comparison of mass spectra with those in the NIST 2005 and Wageningen Mass Spectral Database of Natural Products MS libraries. Experimentally obtained linear retention indices (LRI) were used as additional criteria for compound identification. Peak area quantification of individual compounds was carried out in selected (single) ion monitoring (SIM) mode.

All peaks that did not occur in the blank controls were analysed further. The individual peak area of each compound was related to the fresh weight of the aboveground 
parts of each plant sample and used for further characterization of the different plant groups using statistical analysis. All samples were analysed consecutively, after cleaning and tuning the mass spectrometer for best performance and to limit changes in sensitivity of the machine during the analysis.

Pieris brassicae oviposition preference experiment in cages We conducted dualchoice oviposition bioassays in cages in a greenhouse compartment $\left(21 \pm 1{ }^{\circ} \mathrm{C}, 50-70 \%\right.$ $\mathrm{RH}, \mathrm{LD} 16: 8$ ) to test whether ovipositing $P$. brassicae females discriminate between different $B$. nigra accessions and between egg-free and egg-laden plants. Egg-laden plants were infested as described above $24 \mathrm{~h}$ before the experiment. Thereafter, the eggs were removed to minimize visual or other egg-related cues influencing oviposition choice. Then the plants were offered to female butterflies, which had previously mated only once and had been kept without a host plant for two days to increase egg-laying motivation. Females subjected to these pre-treatments made oviposition choices within $24 \mathrm{~h}$. A single female was placed into a cage $(75 \times 75 \times 115 \mathrm{~cm})$ with two $B$. nigra plants. We tested the following four combinations: (1) SF19 control vs. SF48 control; (2) SF19 eggs vs. SF48 eggs; (3) SF19 control vs. SF19 eggs; and (4) SF48 control vs. SF48 eggs. Each combination was tested ten times, with ten different butterflies. After the $24 \mathrm{~h}$-choice period, photos of all eggs on the plants were made. The number of eggs was counted using ImageJ and GIMP software.

Egg parasitoid preference experiment in a Y-tube olfactometer Attraction of egg parasitoids to volatiles of the two B. nigra accessions was tested by offering simultaneously (i) odour of an egg-laden and egg-free plant of the SF19 line or (ii) odour of an egg-laden and egg-free plant of the SF48 line. We tested the wasps' response to seven plant pairs of each line. For these assays, we used three different T. evanescens isofemale lines (see above). Bioassays were conducted in a dynamic airflow Y-tube olfactometer (for details see Fatouros et al. 2012). This olfactometer was designed to track minute wasps like Trichogramma spp. that were released in groups. We have previously shown that group release does not influence the behaviour of Trichogramma wasps (Fatouros et al., 2012). The plants were placed into glass jars, and plant odours were introduced into the Y-tube olfactometer via PTFE Teflon tubes (air flow: $400 \mathrm{ml} \mathrm{min}^{-1}$ ). Ten female wasps were released as a group into the base of the Y-tube. Their choice 
for one or the other odour (plant) source was assessed. Thirty minutes after release, the numbers of wasps that walked into the right or left glass bulb at the end of the Y-tube, and the number of non-responding wasps were counted. All wasps were then removed, the airflow was switched between jars to compensate for unforeseen left/right bias, and a new group of wasps was released towards the same plant pair. Each plant pair was used to test all three parasitoid isofemale lines mentioned above. A replicate was considered successful, if at least six out of ten wasps made a choice. If this was not the case, a new plant pair was tested with a new group of wasps.

Semi-field experiment on larval parasitism rates In a semi-field experiment, we determined whether parasitism rates of $P$. brassicae larvae feeding on SF19 or SF48 plants differ from each other. In the field, the butterfly larvae are frequently parasitised by the wasp C. glomerata (Poelman et al., 2009b). We also investigated whether the larval parasitism rates differ in dependence of prior egg deposition by $P$. brassicae on the same plant, on which the larvae fed. Previous laboratory bioassays revealed that the attraction of $C$. glomerata wasps to $B$. nigra plants infested by $P$. brassicae neonate caterpillars is dependent on whether the neonates are feeding on egg-free plants or previously egg-laden ones (Pashalidou et al., 2015c). Here, we tested whether parasitism rates determined under semi-field conditions support the previous laboratory findings.

Potted SF19 and SF48 plants were placed outside at the experimental farm of Wageningen University (Unifarm), The Netherlands. Twelve plants were left without eggs when placing them into the field site, while twelve further plants had been infested with 30 P. brassicae eggs $24 \mathrm{~h}$ before.

All plants were watered, and egg-laden plants were checked for hatched caterpillars daily. When caterpillars hatched from egg-laden (EF, eggs and feeding damage) plants, they were transferred to other leaves from the same plant. To infest previously untreated (egg-free) plants with caterpillars, newly hatched caterpillars from the rearing were used and placed on leaves of egg-free ( $F$, feeding damage only) plants. Neonate caterpillars feeding on these plants were exposed to the natural $C$. glomerata population.

After the caterpillars had fed on EF and F plants for one day, the plants together with the caterpillars were brought inside. The plants were then carefully checked to remove any adult $C$. glomerata wasps and then placed under a net to protect the caterpillars on the plants from free-flying $C$. glomerata parasitoids. The number of $C$. glomerata larvae 
or eggs inside the $P$. brassicae caterpillars were counted by dissecting the caterpillars within five days.

Common-garden experiment Effects of $P$. brassicae egg parasitism rates on plant fitness of the different brass accessions were tested in a common-garden experiment. Details are described in the Supplementary information.

Statistical analysis All analyses were performed using R (R Core Team, 2016).

Statistical analysis of quantitative leaf volatile emission. The emission rates of plant volatiles were analysed by cluster analysis similar to Rusman et al. 2019. For cluster analysis, data were averaged per treatment, $\log 10$ transformed, and range scaled. Clustering was done using Euclidian distances and Ward's clustering criterion. Bootstrapping was done using 1,000 bootstrap replicates. We used the pvclust package for these analyses (Suzuki and Shimodaira, 2006). Resulting approximately unbiased (AU) Pvalues were displayed at the nodes below the first split. The values are given between 0 and 100 with values above 95 considered statistically significant. Heat maps were produced with MetaboAnalyst 4.0 with default settings, but without standardization (Xia et al., 2015). The analysis showed the variable importance in the projection (VIP) of each variable (in this case, the different compounds).

Subsequently, single VOCs were analysed to test if egg treatment, accession or the interaction between the two affects the emitted quantities. We first tested the $\log 10$ transformed data of peak areas for normality and homogeneity of variance across all treatments and accessions (SF19 control, SF19 eggs, SF48 control, SF48 eggs). If the Shapiro-Wilk test and Levene test showed normality and variance homogeneity, respectively, we used a two-way ANOVA to test for the effect of egg treatment and plant accession. After using an ANOVA, we conducted Student's t-tests as post-hoc tests if a significant difference was found by the ANOVA. If plant accession was shown to exert a significant effect on the quantity (peak area) of the respective VOC, we tested SF19 control vs. SF48 control and SF19 eggs vs. SF48 eggs. If the egg treatment exerted a significant effect, we tested SF19 control vs SF19eggs and SF48 control vs. SF48 eggs. For those cases, where normality or homogeneity of variance were not given, we used a generalized linear model (GLM) to test for the effect of egg treatment and plant accession. To ascertain which distribution (family in the GLM function) to use, 
several tests were applied. First, we tested the untransformed data for inverse Gaussian distribution, using the ig_ test function of the goft package. If inverse Gaussian distribution was not supported, we tested the $\log 10$ transformed data for inverse Gaussian distribution. The quantitative data of only three volatiles did not fit an inverse Gaussian distribution. Two of those fitted a gamma distribution instead. Quantities of one compound (7a-isopropenyl-4,5-dimethyloctahydro-inden-4-yl)methanol (IDMOM) did not fit either distribution, and when tested in inverse Gaussian and gamma distributions, in both cases no $P$-values approached significance levels. After using a GLM, we conducted Mann-Whitney U tests as post-hoc tests if a significant difference was found by the GLM. If plant accession was shown to be significant, we tested SF19 control vs. SF48 control and SF19 eggs vs. SF48 eggs, if egg treatment was significant, we tested SF19 control vs SF19 eggs and SF48 control vs. SF48 eggs.

A Bonferroni correction was used for both, Student's t-test and Mann-Whitney U test, to adjust the global $\alpha$ from previous 0.05 to $0.025(0.05 / 2)$, as two tests were conducted, only $P$-values less than 0.025 were considered significant.

Statistical analysis of insect behavioural responses. The attraction of either $P$. brassicae or T. evanescens females to egg-laden plants was investigated using logistic regression [i.e. a GLM with a binomial distribution and a logit link function] with plant treatment and parasitoid species as fixed factors. A quasi-binomial distribution was fitted in the model due to overdispersion. The fraction of eggs laid by butterflies was used as response variable, with plant treatment (clean or eggs) as fixed effect variable. Then the number of eggs laid on egg-infested plants over the number of total eggs laid per replicate ( $P$. brassicae) or number of wasps choosing egg-induced plants over the total number of wasps choosing (T. evanescens) was used as response variable with plant accession (and additionally wasp line for T. evanescens) as fixed effects. To further test for each dual choice treatment whether there was a significant preference for one of the offered plant treatments, we tested H0: logit $=0$.

Statistical analysis of larval parasitism rates. Cotesia glomerata parasitism rates and numbers of parasitoids per caterpillar after $24 \mathrm{~h}$ of caterpillar exposure in the field experiments were first checked for the influence of the plant infestation date, using generalized linear models (binomial for parasitism rate and Gamma distribution for number of parasitoids per caterpillar). Because plant infestation date was a significant factor in the models, generalized linear mixed-effect models (GLMM), including date of infestation 
as random factor were used to test the other variables (egg treatment, plant accession, and or the interaction between the two) for their influence on the parasitism rate and number of parasitoids per caterpillar. Furthermore, the parasitism rate and number of parasitoids per caterpillar were related to the number of caterpillars per plant. Those relative means were also used to generate the figures. Post-hoc tests were performed using Estimated Marginal Means (EMMs), function from the emmeans package.

\section{Acknowledgments}

We thank people of Unifarm Wageningen for setting up the field sites and providing plants. André Gidding, LéonWesterd, Joop Woelke and Frans van Aggelen of the lab of Entomology of Wageningen University for culturing insects. The DAAD (57044990), The Dutch Research Council (NWO/TTW Vidi grant 14854 to N.E.F.) and the German Research Foundation (CRC 973; www.sfb973.de to M.H.) for funding. 
3.A Supplementary material 


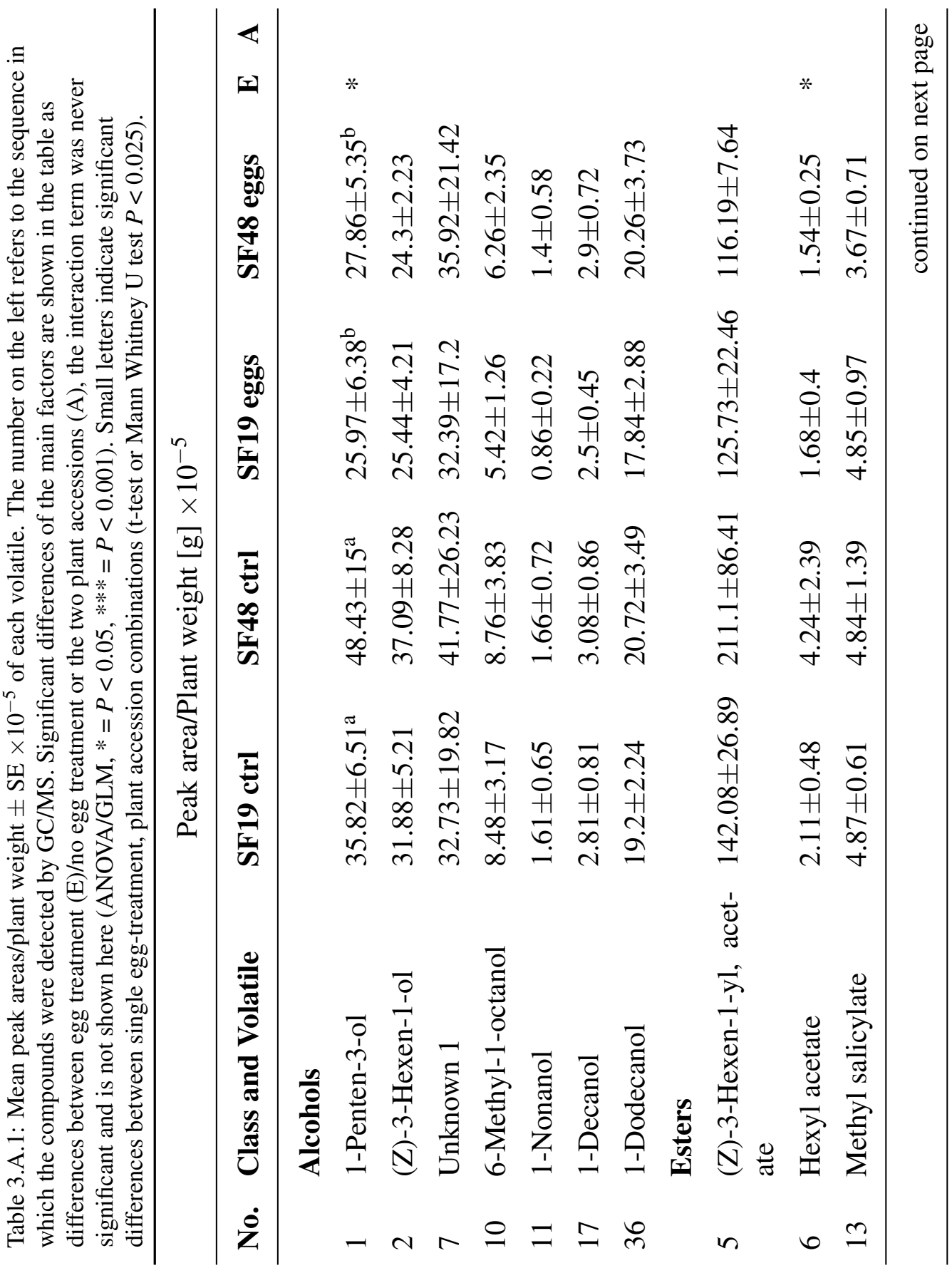




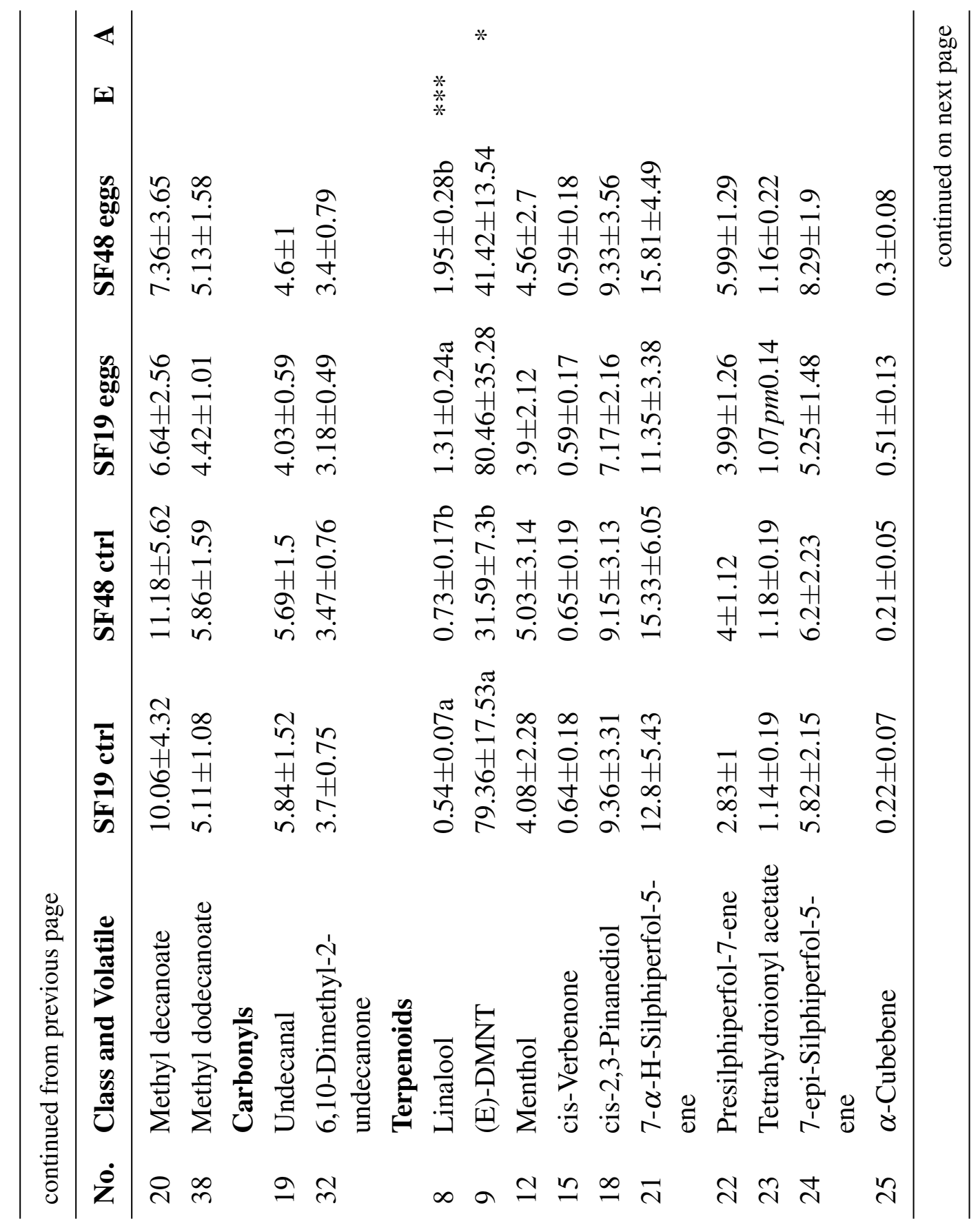




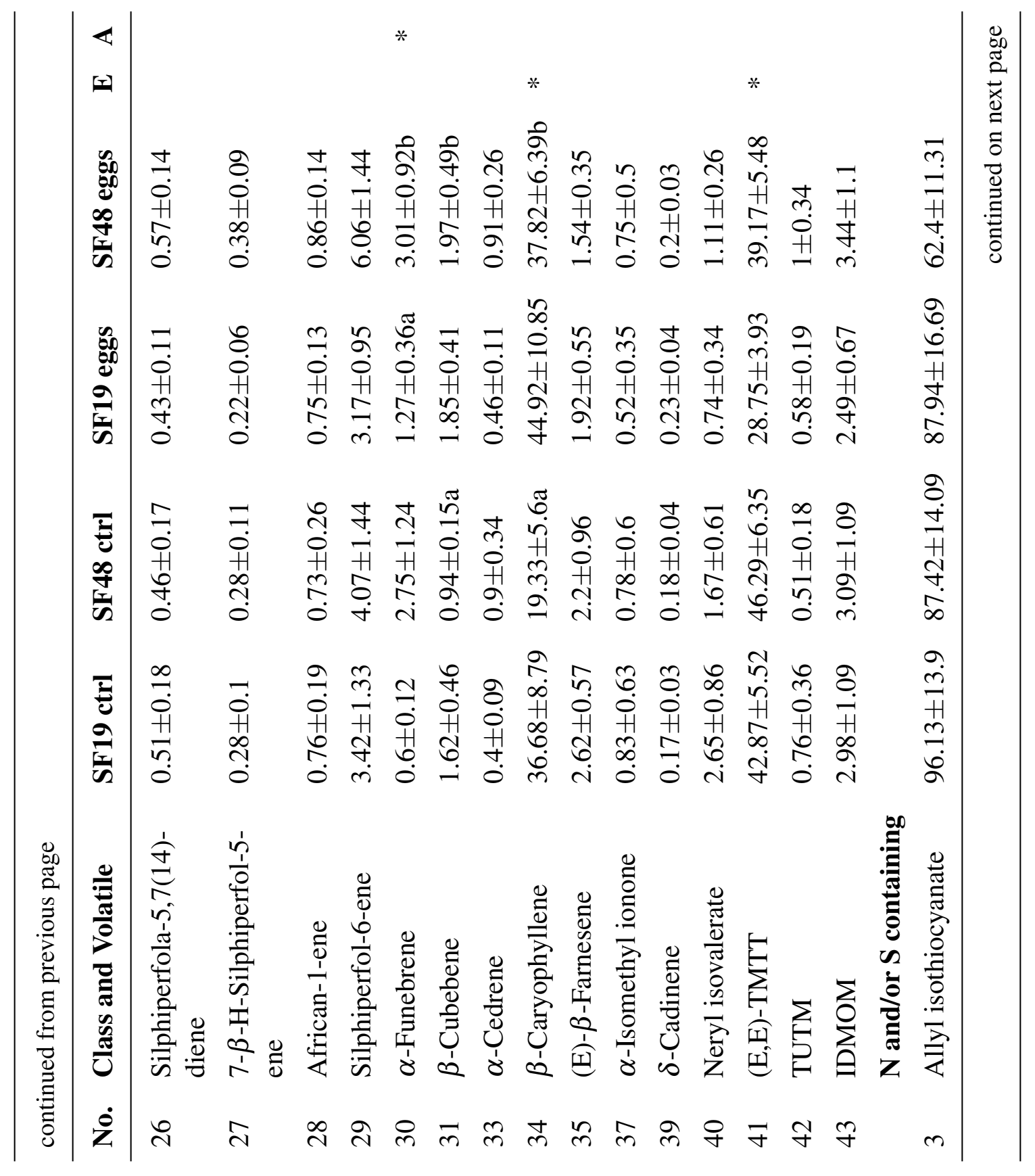




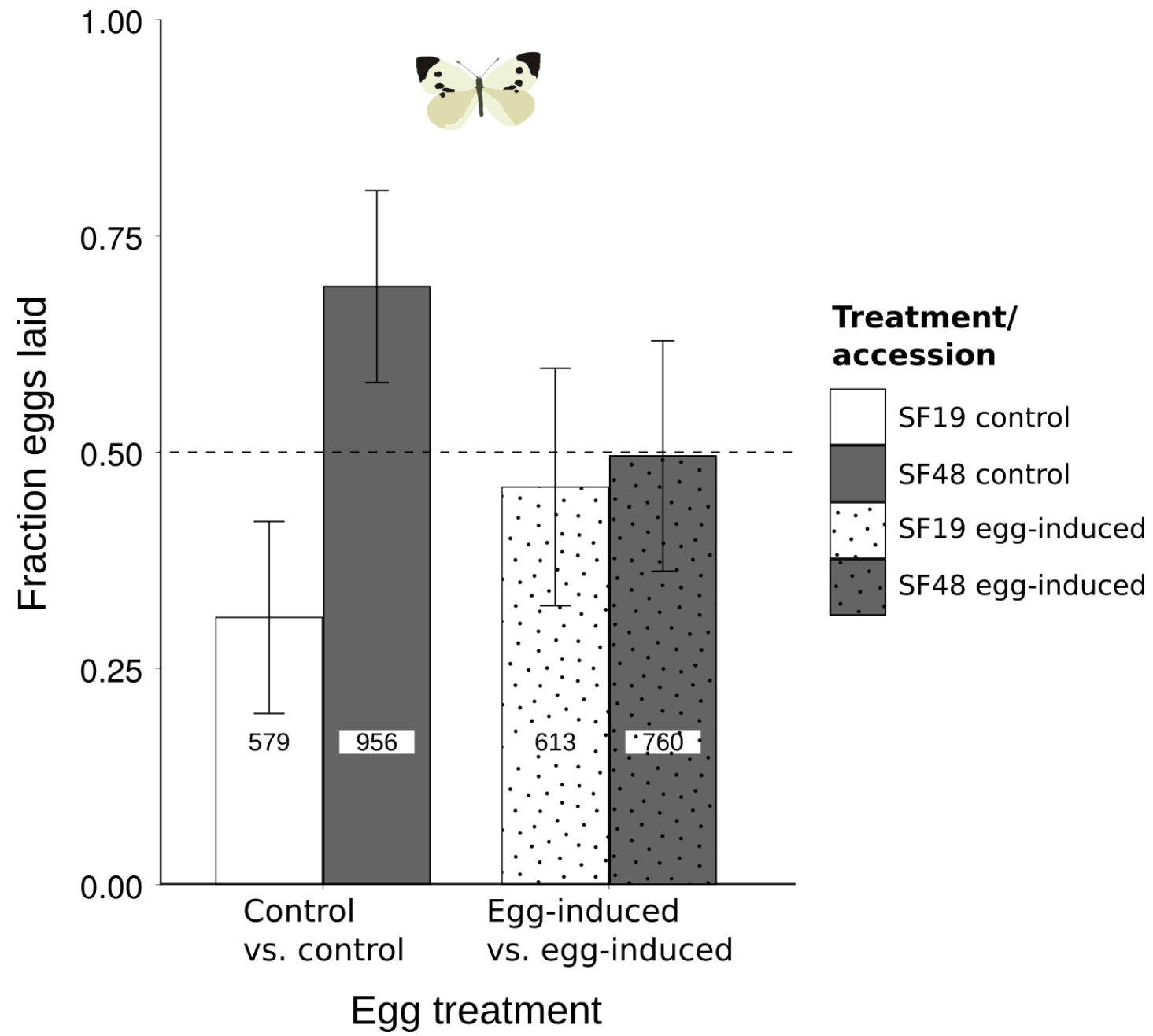

Figure 3.A.1: Behavioural responses to $B$. nigra plants by gravid P. brassicae. Mean $( \pm \mathrm{SE})$ fraction of eggs deposited. Oviposition choice by $P$. brassicae butterflies between uninfested SF19 and uninfested SF48 or egg-infested SF19 and egg-infested SF48 plants. No significant results were obtained. The total number of eggs laid by females onto the plants are given in the bars. 


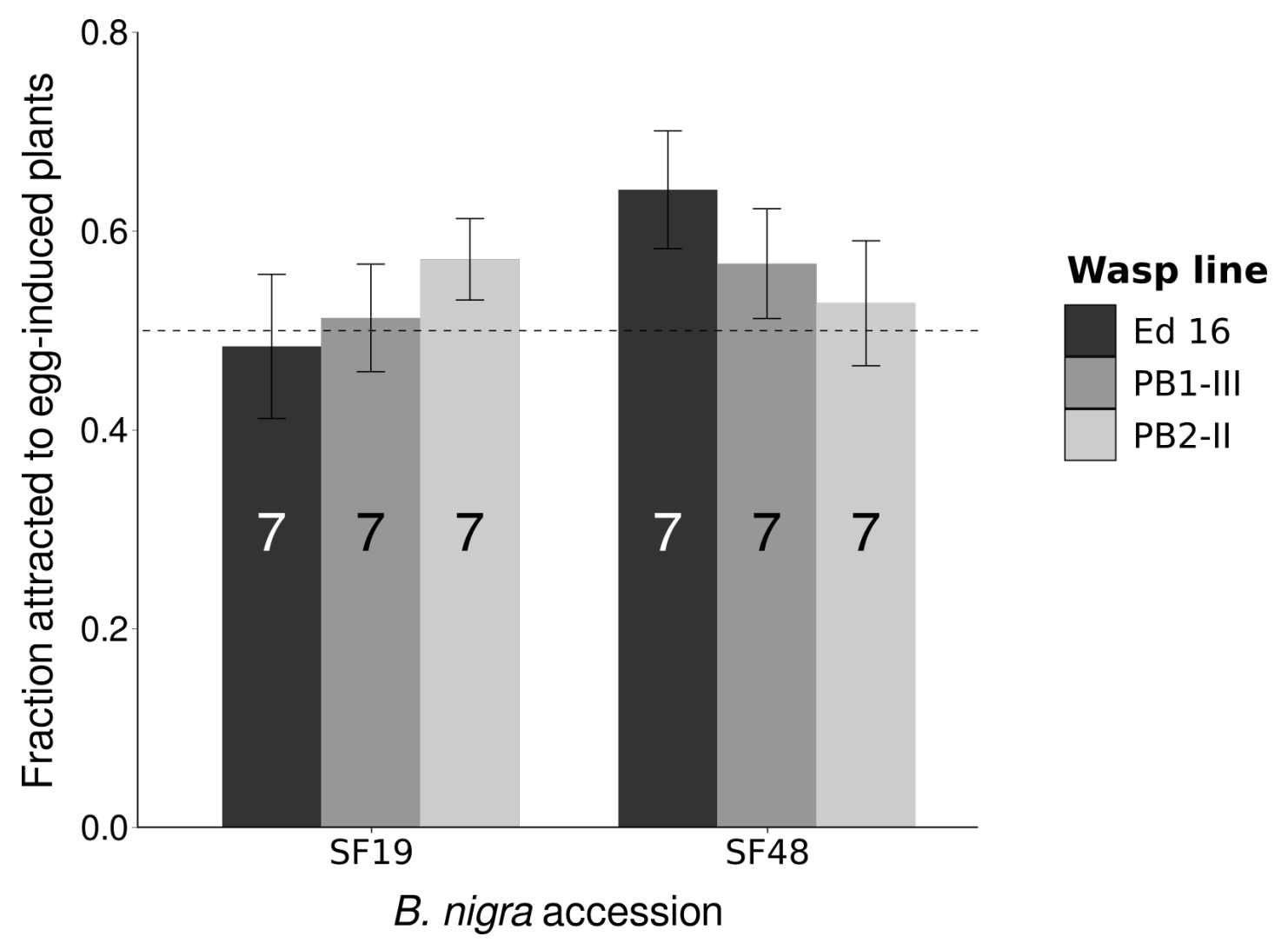

Figure 3.A.2: Three different isofemale lines of T. evanescens tested in a Y-tube olfactometer. Mean $( \pm \mathrm{SE})$ fraction of wasps choosing plants with eggs over uninfested plants after $24 \mathrm{~h}$ of egg-induction. The 0.5 value is marked in the graph. Numbers represent the number of plant pairs tested. For each plant pair ten wasps of each line were tested. No significant results were obtained.

Table 3.A.2: Expression of HR in the experimental plants used for behavioural tests. This data is related to Figure 3.3 and Figure 3.A.1; 'no HR data': these plant individuals were not checked for HR-like symptoms.

\begin{tabular}{lllll}
\hline Experiment & Accession & HR + & HR - & No HR data \\
\hline Pieris oviposition & SF19 & 9 & 8 & 5 \\
& SF48 & 10 & 8 & 4 \\
Parasitoid response in Y-tube & SF19 & 1 & 2 & 4 \\
& SF48 & 1 & 2 & 4 \\
\hline
\end{tabular}




\section{Field experiments}

In two consecutive years, $B$. nigra plants were planted in a large-scale common-garden experiment. The whole field was set up close to the University of Wageningen, Wageningen, The Netherlands. The field was surrounded with a border of two rows of B. nigra plants. In 2013, SF19 and SF48 were planted. To increase diversity of plant accessions we included two further self-fertilized accessions in 2014, i.e. we planted SF19, SF25, SF29 and SF48. Three planting time points were used in both years. Eight plants of every B. nigra accession was planted in its own plot (Figure 3.A.3). Plots were separated from each other by 3-metre-wide grass stripes, sown at the time of establishing the plots. Four plots formed one row of the experimental field. In 2013 the field consisted of twelve rows, four of which were planted at the same time. In 2014, the field consisted of fifteen rows, five of which were planted at the same time. This approach was chosen to be able to handle the number of plant observations per week, while having the plants at the same age when egg treatment started. Experimental plants were surrounded with four satellite plants each, mainly to lower the risk of caterpillars crawling from one treatment to another. Plants were treated in four ways: (1) Control plants (C), were not infested with any $P$. brassicae eggs at the start of the experiment and no caterpillars were transferred to them later. (2) Egg infested (E) plants received P. brassicae eggs but no caterpillar feeding damage. (3) Egg infested and feeding damaged (EF) plants received $P$. brassicae eggs, and caterpillars were left to feed on the plants. (4) Feeding damage only (F) plants received no $P$. brassicae eggs but caterpillars were transferred to them after they hatched on E plants, as well as from the rearing, if the number of hatching caterpillars was not sufficient. Two plants of each treatment were planted in a plot, one of which was covered with a gauze net to prevent egg and larval predators and parasitoids from reaching the eggs/larvae. Plants covered with gauze are referred to with an additional $P$ (protected) $\left(\mathrm{E}_{\mathrm{P}}, \mathrm{EF}_{\mathrm{P}}, \mathrm{F}_{\mathrm{P}}\right.$ and $\left.\mathrm{C}_{\mathrm{P}}\right)$. After the caterpillars reached larval stage 2 the nets were taken off the plants. For more details see Griese et al. (2017). 


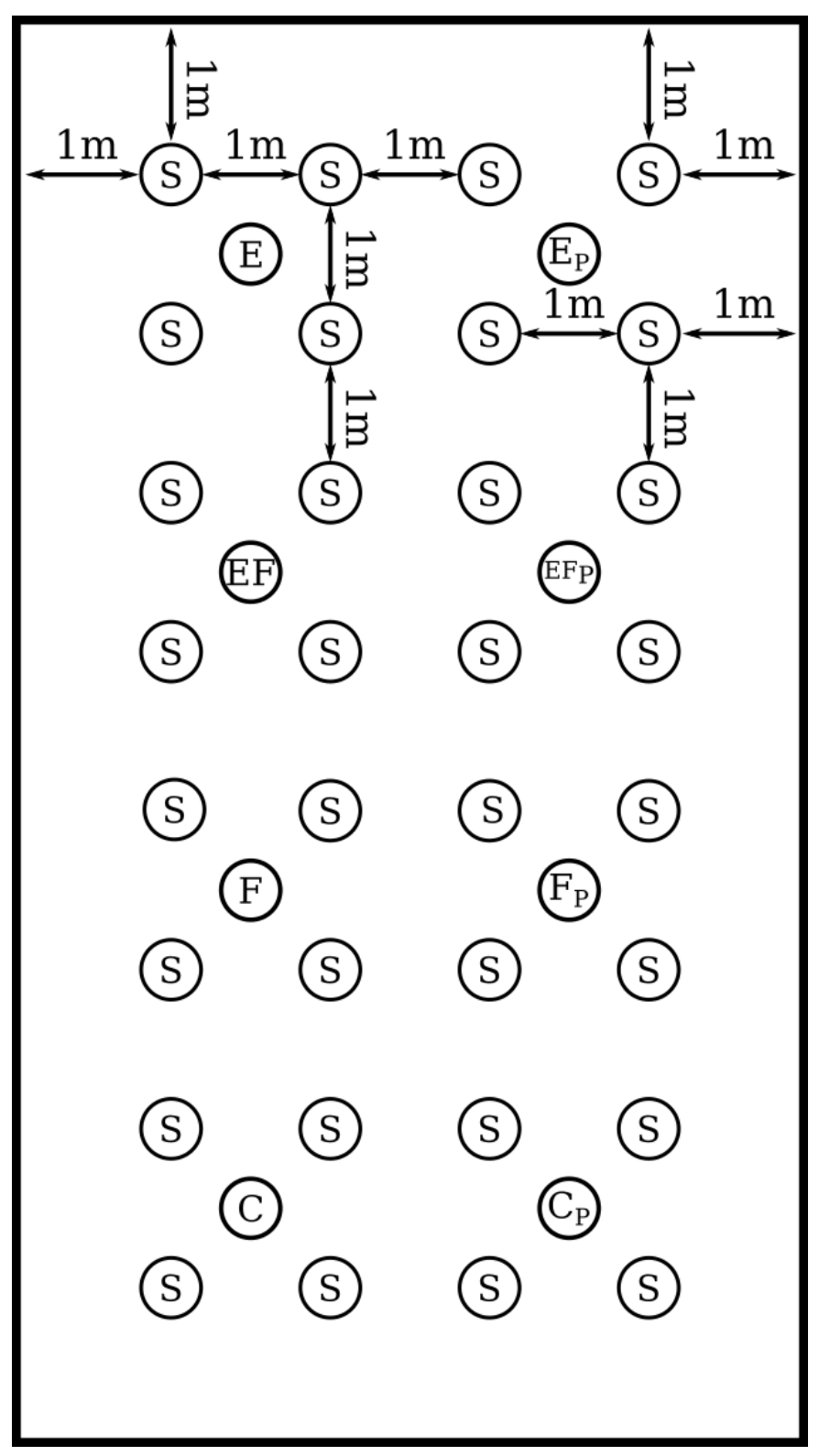

Figure 3.A.3: Single field plot of the field experiment. Each experimental B. nigra plant is surrounded by four satellite plants $(\mathrm{S})$ to prevent caterpillars from crawling from one treatment to another. Plants either received only eggs and no caterpillar feeding damage (E), eggs and feeding damage (EF), only feeding damage (F) or were left untreated for control (C). Each treatment was duplicated as unprotected or protected $(\mathrm{P})$ where a net covered the plant to prevent egg and larval parasitism. 


\section{Pieris brassicae egg and larval survival}

After infesting $B$. nigra plants with $P$. brassicae egg clutches in the common garden experiment, the survival of the eggs and later caterpillars was monitored. The egg survival was checked by the number of hatched larvae (caterpillars hatched between five and ten days after oviposition), while later caterpillar survival was measured once a week by counting the number of caterpillars present on the plants.

\section{Plant fitness assessment}

All covered and non-covered plants from the different treatments E, EF, F and C were harvested once they developed seeds. Seeds of each plant were cleaned and weighed using a microbalance with a $1 \mathrm{mg}$ accuracy. A sample of 100 seeds was counted using a CONTADOR 'E' seed counter (www.pfeuffer.com/contador.html), and a balance to weigh those 100 seeds. This weight was used to calculate the weight of a single seed. The same balance was also used to weigh all seeds.

\section{Statistical analysis}

Egg parasitism rates on B. nigra accessions was tested using $\chi^{2}$ test. Egg hatching rates on covered and uncovered plants were tested for significant differences using a GLM with quasibinomial distribution, followed by EMEANS as post-hoc test. Plant seed weight was compared between accessions and treatment using general linear mixed effect models (GLMM). The planting/egg infestation date of the plants was used as the random factor. 

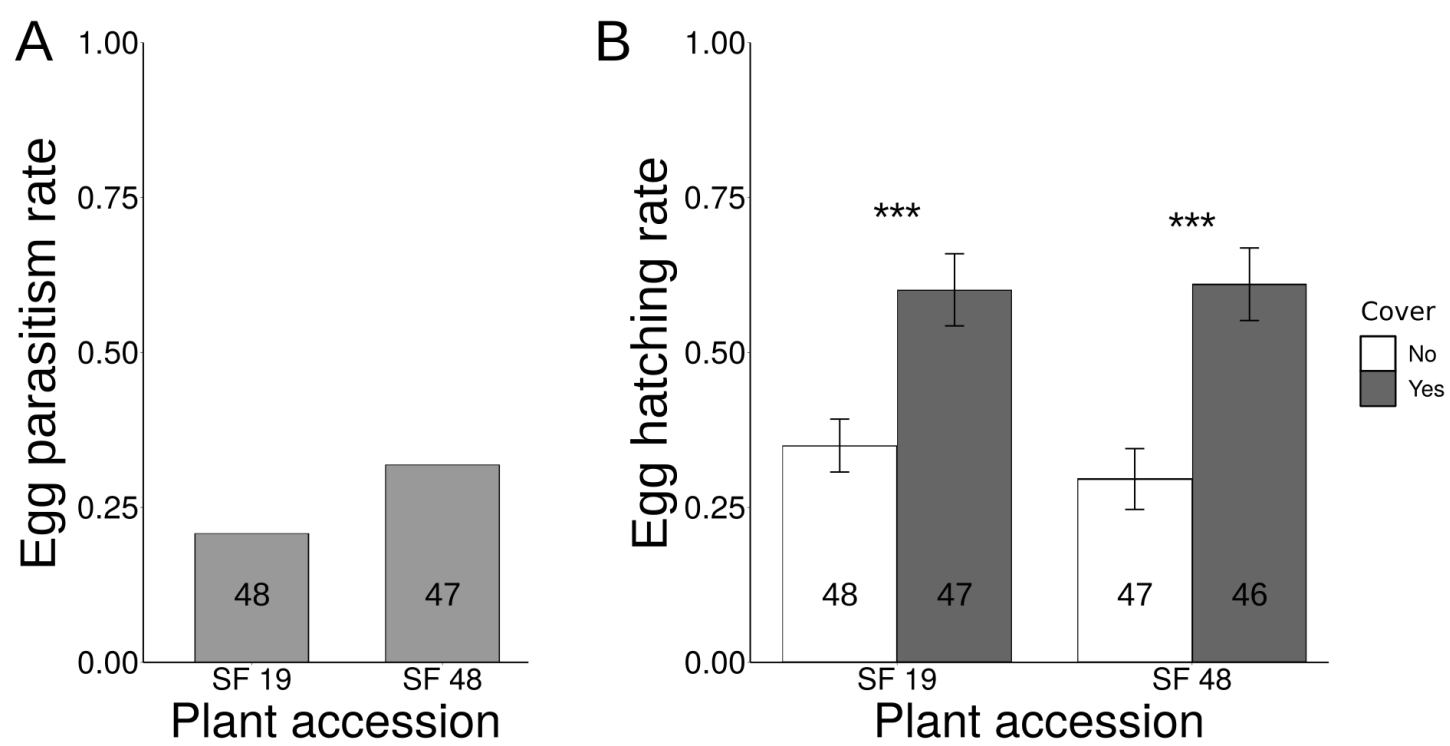

Figure 3.A.4: Field egg parasitism and egg hatching (Mean \pm SE) rates. A) Trichogramma sp. egg parasitism rate as parasitised egg clutches per total egg clutches. Non-significant differences are marked as ns $\left(\chi^{2}\right.$ test: $\left.P>0.05\right)$. B) Egg hatching rates as hatched caterpillars per eggs per egg clutch. Significant differences between covered and uncovered plants are indicated by asterisks $* * *$ (GLM, EMMEANS: $P<0.001$ ). 
A

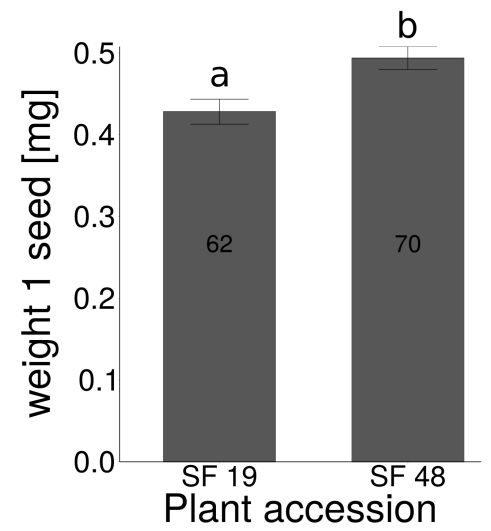

C

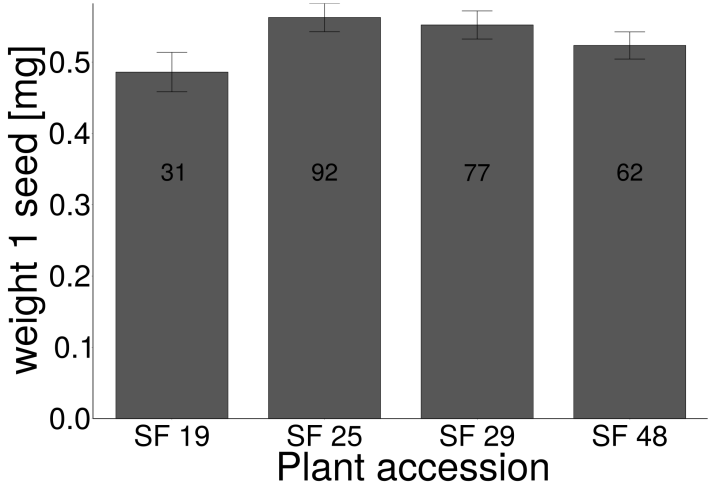

B

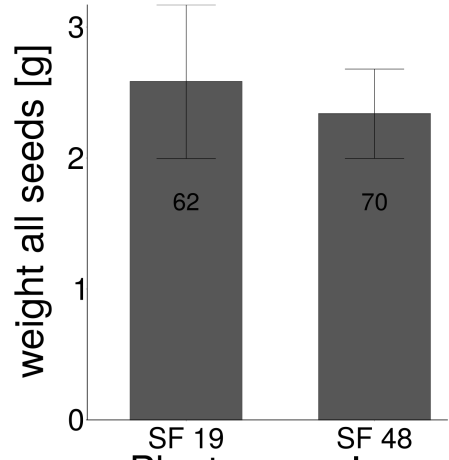

D

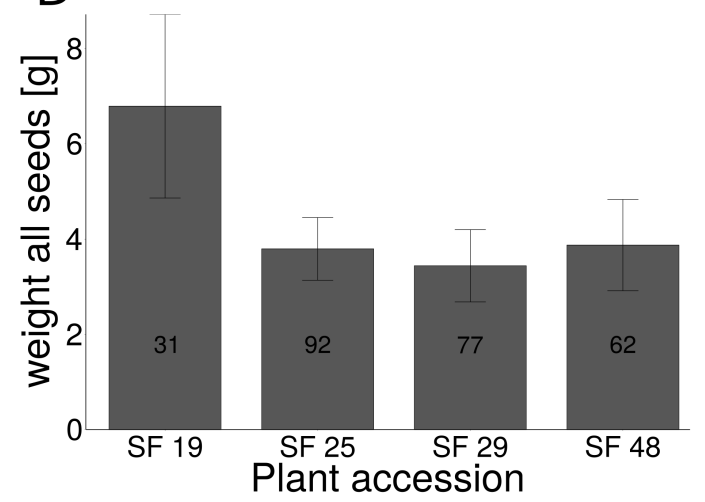

Figure 3.A.5: Seed weight (Mean \pm SE) differences between B. nigra accessions of two consecutive years. In 2013, only SF19 and SF48 plants were planted. In 2014, SF19 and SF48 were planted again, as well as the accessions SF25 and SF29. A) Weight of single seed per plant, year 2013. B) Total seed weight of all seeds per plant, year 2013. C) Weight of single seed per plant, year 2014. D) Total seed weight of all seeds per plant, year 2014. Small letters indicate significant differences between plant accessions (GLMM: $P<0.05$ ). Only significant differences are shown. 


\section{Chapter 4}

Plant responses to butterfly oviposition partly explain preference-performance relationships on different brassicaceous species

Eddie Griese, Ana Pineda, Foteini G. Pashalidou, Eleonora Pizzaro Iradi, Monika

Hilker, Marcel Dicke \& Nina E. Fatouros

Published in: Oecologia (2020), 192, pp. 463-475

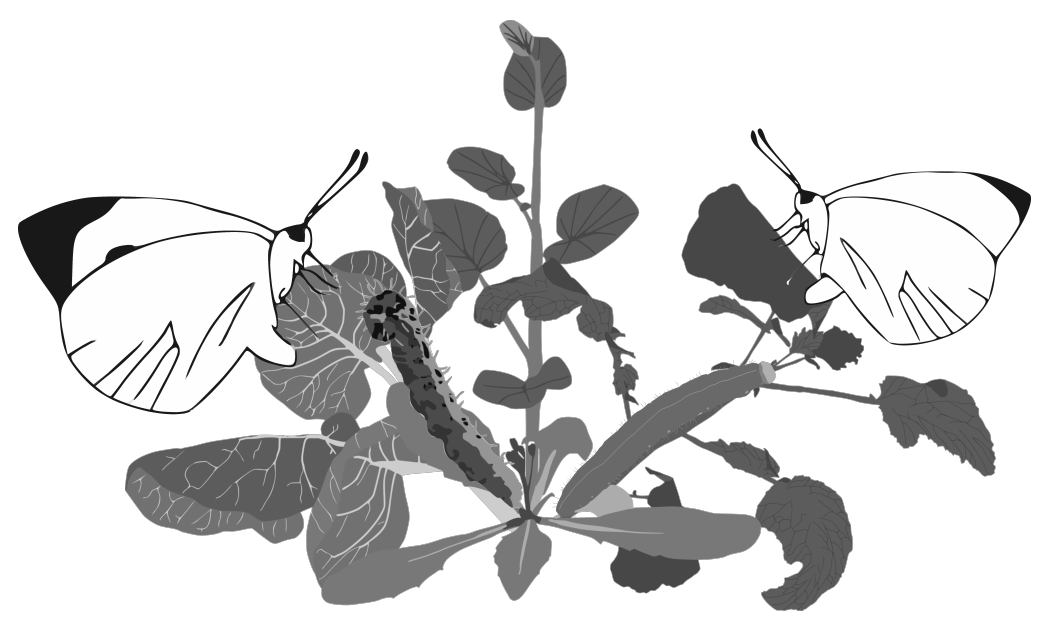




\section{0}

\section{Abstract}

The preference-performance hypothesis ( $\mathrm{PPH})$ states that herbivorous female insects prefer to oviposit on those host plants that are best for their offspring. Yet, past attempts to show the adaptiveness of host selection decisions by herbivores often failed. Here, we tested the PPH by including often neglected oviposition-induced plant responses, and how they may affect both egg survival and larval weight. We used seven Brassicaceae species of which most are common hosts of two cabbage white butterfly species, the solitary Pieris rapae and gregarious P. brassicae. Brassicaceous species can respond to Pieris eggs with leaf necrosis, which can lower egg survival. Moreover, plant-mediated responses to eggs can affect larval performance. We show a positive correlation between $P$. brassicae preference and performance only when including the egg phase: 7-dayold caterpillars gained higher weight on those plant species which had received most eggs. Pieris eggs frequently induced necrosis in the tested plant species. Survival of clustered $P$. brassicae eggs was unaffected by the necrosis in most tested species and no relationship between $P$. brassicae egg survival and oviposition preference was found. Pieris rapae preferred to oviposit on plant species most frequently expressing necrosis although egg survival was lower on those plants. In contrast to the lower egg survival on plants expressing necrosis, larval biomass on these plants was higher than on plants without a necrosis. We conclude that egg survival is not a crucial factor for oviposition choices but rather egg-mediated responses affecting larval performance explained the preference-performance relationship of the two butterfly species.

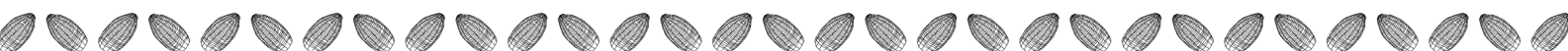

\section{Introduction}

Host-plant selection for oviposition by insect females is a decisive step in establishing a new herbivore generation (J. Thompson, 1988; J. N. Thompson, 1988; J. Thompson and Pellmyr, 1991; Gripenberg et al., 2010). The preference-performance hypothesis $(\mathrm{PPH})$ or 'mother-knows-best' hypothesis states that natural selection favours those insect females which prefer hostplants where the offspring performs best, especially when immature stages are less mobile than adults. A good host plant is usually characterized 
either by a high nutritional value, reduced defence, and/or by enemy-free space (Mayhew, 1997; Mayhew, 2001; Jaenike, 1990; Craig and Ohgushi, 2002; Gripenberg et al., 2010). In addition to host-plant quality and presence of natural enemies, various factors such as local host-plant abundance or distribution patterns of host plants shape oviposition preference and larval performance (Wiklund and Friberg, 2008; Wiklund and Friberg, 2009; Friberg et al., 2015). While the PPH is supported by many studies of butterflies and moths (J. Thompson, 1988; J. N. Thompson, 1988; J. Thompson and Pellmyr, 1991; Harris et al., 2001; Forister, 2004; Forister et al., 2009), numerous others failed to do so (J. N. Thompson, 1988; Jaenike, 1990; Mayhew, 1997; Gripenberg et al., 2010; Scheirs et al., 2000; König et al., 2016).

Yet, the vast majority of innumerable laboratory studies testing the PPH did not consider that plants can activate defences in response to egg deposition. Research has provided evidence that numerous plant species across highly diverse taxa defend against egg depositions of various insect species (Hilker and Fatouros, 2015). Plants are capable of killing eggs (Fatouros et al., 2016). For example, egg-induced formation of a neoplasm (Petzold-Maxwell et al., 2011) or by hypersensitive response (HR)-like necrosis at the oviposition site (Shapiro and DeVay, 1987; Fatouros et al., 2014; Griese et al., 2017) may result in egg detachment from the plant or egg dehydration. Furthermore, a plant can kill insect eggs by growing tissue that crushes the eggs (Karban, 1983; Mazanec, 1985; Aluja et al., 2004; Desurmont and Weston, 2011). In addition, plants can receive insect eggs as early 'warning cues' of impending herbivory and reinforce or prime their defences against the subsequently feeding larvae (Pashalidou et al., 2013; Pashalidou et al., 2015c; Bandoly et al., 2015; Hilker and Fatouros, 2015; Hilker and Fatouros, 2016; Austel et al., 2016; Hilker et al., 2016) . As a consequence, larval performance on initially egg-infested plants may be worse than on egg-free plants (Hilker and Fatouros, 2016). Additionally, but less frequently shown so far, egg deposition can suppress plant defences against larvae (Reymond, 2013). The effects of egg deposition on subsequent plant defences against larvae have been mainly overlooked until recently, with most studies on larval performance conducted by placing larvae onto an egg-free host plant (Hilker and Fatouros, 2015; Hilker and Fatouros, 2016).

The insect oviposition mode can have a significant impact on egg survival and larval performance. When eggs are laid in clusters, neonate larvae often show gregarious feeding behavior, which benefits offspring performance in some insect species (Denno 
and Benrey, 1997; Clark and Faeth, 1998; Fordyce, 2003; Allen, 2010; Desurmont et al., 2014; Martinez et al., 2017). On the other hand, many herbivorous insects lay single eggs, spreading them over a larger area, possibly as a means of reducing predation risk and competition (Root and Kareiva, 1984; Nufio and Papaj, 2001). Egg-induced plant defence affecting larval performance is especially known for insect species laying eggs in clutches (Hilker and Fatouros, 2015). However, plant response to singly laid eggs of Manduca sexta reinforces the defence against M. sexta larvae (Bandoly et al., 2016). It remains to be elucidated whether the egg-laying mode (single eggs vs. egg clutches) affects egg-induced plant defence targeting the eggs and how this in turn depends on the plant species receiving the eggs.

The aim of this study is to elucidate whether there is a positive outcome of the PPH when not only considering relationships between oviposition preference and larval performance, but also when including egg survival rates and egg-induced changes in plant suitability for feeding caterpillars. Therefore, we investigated oviposition preference, egg survival and larval performance of Pieris brassicae and P. rapae on seven Brassicaceae species. Pierid butterflies have coevolved over 90 million years with their host plants in the order Brassicales (Wheat et al., 2007; Edger et al., 2015). Both butterfly species are known to use various wild and cultivated brassicaceous plants as hosts (Feltwell, 1982; Chew and Renwick, 1995; Gols et al., 2011), whereby P. rapae can include also non-brassicaceous plants in their diet (Friberg et al., 2015). Pieris caterpillars are well adapted to Brassicaceae by their ability to detoxify glucosinolates, plant secondary metabolites characteristic for this plant taxon (Hopkins et al., 2009). While $P$. rapae lays single eggs on plants, $P$. brassicae lays egg clutches containing up to 200 eggs (Feltwell, 1982) (Supplementary Figure 4.A.1). Egg deposition by these Pierid species is known to induce an HR-like leaf necrosis in several host plant species (Fatouros et al., 2016). Additionally, previous egg deposition by P. brassicae on brassicaceous plant species negatively affects larval performance (Geiselhardt et al., 2013; Pashalidou et al., 2013; Pashalidou et al., 2015a; Bonnet et al., 2017). It remains unknown so far how egg deposition of the conspecific solitary species $P$. rapae affects subsequently feeding larvae through egg-mediated plant responses.

We specifically addressed the following questions: (1) is oviposition choice of two pierid species, $P$. brassicae and $P$. rapae affected by the plant species' capability to activate an egg-killing response (i.e., HR-like necrosis)? (2) Do females prefer to oviposit 
on plants on which their eggs show highest survival rates and larvae perform best? (3) Do plant-mediated effects of eggs change caterpillar performance (i.e., defence priming) and if so (4) do they correlate with butterfly oviposition choices?

\section{Results}

Oviposition preference Gregarious species Although P. brassicae females distributed their egg clutches unevenly between plants $\left(\chi^{2}=19.65\right.$, df $=7, P=0.04$, GLM, Figure 4.1a), a post hoc test did not reveal a significant preference (Supplementary Table 4.A.1). Arabidopsis thaliana, which was in the flowering stage (in contrast to all other plant species), did not receive any egg clutches from $P$. brassicae in this setup. Oviposition choices of $P$. brassicae did not correlate with plant fresh weight $(\mathrm{S}=1720.7, \rho=0.25$, $P=0.24$, Spearman correlation, Supplementary Figure 4.A.2).

Soltiary species Pieris rapae females significantly preferred to oviposit on $B$. nigra over all other seven simultaneously offered plant species (Supplementary Table 1). The plant species chosen least frequently for oviposition were $B$. oleracea and A. thaliana $\left(\chi^{2}=292.67, \mathrm{df}=7, P \leq 0.001\right.$, GLM, Figure $\left.4.1 \mathrm{~b}\right)$. The oviposition preference of $P$. rapae did not correlate with plant fresh weight $(\mathrm{S}=1897.2, \rho=0.18, P=0.41$, Spearman correlation, Supplementary Figure 4.A.2).

Egg survival and effect of plant species and HR-like necrosis Gregarious species Plant species significantly affected $P$. brassicae egg survival $\left(\chi^{2}=20.39\right.$, df $=6, P=$ 0.002 , GLMM, Figure 4.2a). Egg survival was highest on B. montana chosen most for oviposition, while it was lowest on $H$. incana and $R$. sativus compared to all other plants (apart from B. nigra) (Supplementary Table 4.A.2). Five out of the seven tested plant species expressed an HR-like necrosis in response to P. brassicae eggs (Figure 4.2a, Supplementary Table 4.A.2). Overall, induction of HR-like necrosis did not significantly affect egg survival $\left(\chi^{2}=0.41, \mathrm{df}=1, P=0.52\right.$, GLMM), while the effect of HR-like necrosis on survival of $P$. brassicae eggs was plant species specific $\left(\chi^{2}=\right.$ $30.83, \mathrm{df}=4, P<0.001$, GLMM). On $B$. montana, egg survival was much lower on the two HR-expressing plants than on the seven non-HR plants (4.2a, Supplementary Table 4.A.3-4.A.4).

Soltiary species The plant species selected by $P$. rapae females did not significantly 
a)

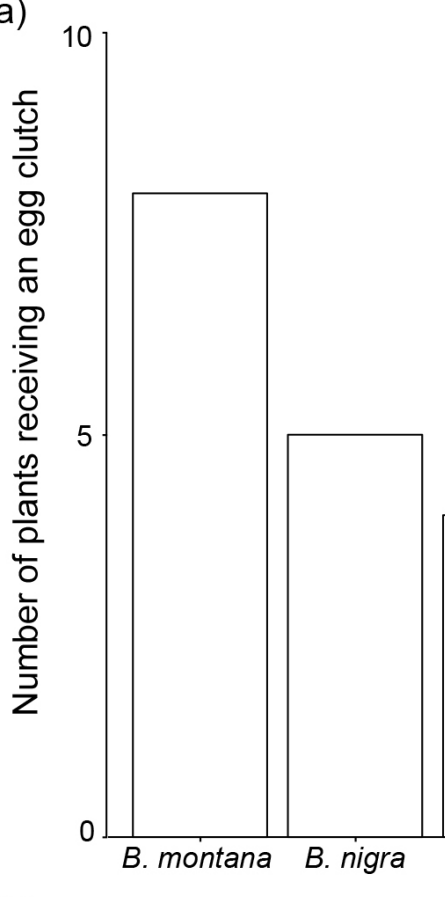

P. brassicae

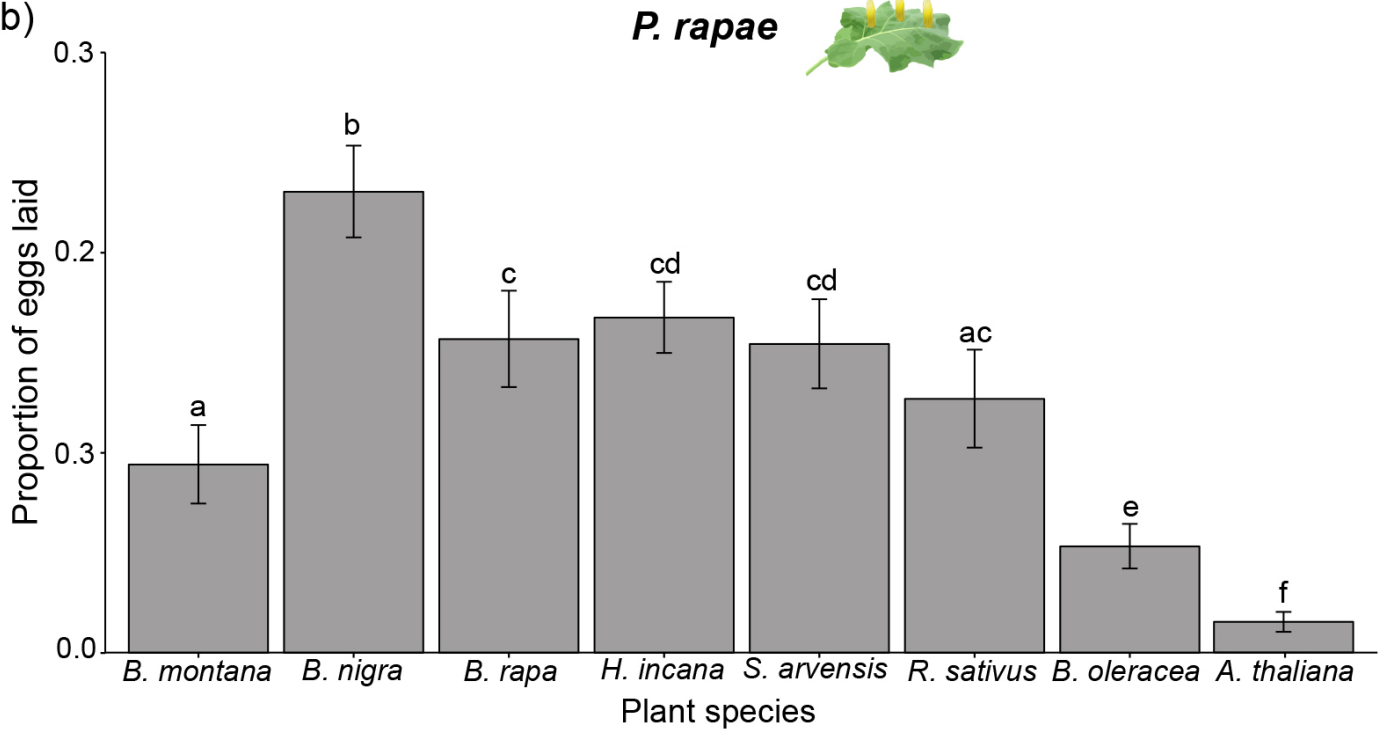

Figure 4.1: Oviposition preference bioassay. a) Number of plants, which received an egg deposition by Pieris brassicae. Female P. brassicae always laid a maximum of one egg clutch per plant; therefore, number of egg-deposited plants is presented. b) Proportion of eggs laid by $P$. rapae on different brassicaceous plant species. The number of single eggs of $P$. rapae was counted per plant species. Mean proportion $\pm \mathrm{SE}$ is given. In total, 18 plants per species were tested in random setups. Small letters indicate significant differences between plant species with $P<0.05$, GLM. 
affect egg survival $\left(\chi^{2}=11.19, \mathrm{df}=6, P=0.08\right.$, GLMM, Figure 4.2b). Six out of the seven tested plant species expressed HR-like necrosis induced by $P$. rapae eggs $(4.2 \mathrm{~b}$, Supplementary Table S2). A significantly higher proportion of $P$. rapae eggs survived on non-HR plants compared to plants expressing HR-like $\left(\chi^{2}=13.58, \mathrm{df}=1, P<0.001\right.$, GLMM). This effect of $P$. rapae egg-induced HR-like necrosis on egg survival was -in contrast to the $P$. brassicae egg-induced response-independent of the plant species $\left(\chi^{2}\right.$ $=4.43, \mathrm{df}=4, P=0.35, \mathrm{GLMM})$ (4.2b, Supplementary 4.A.3-4.A.4).

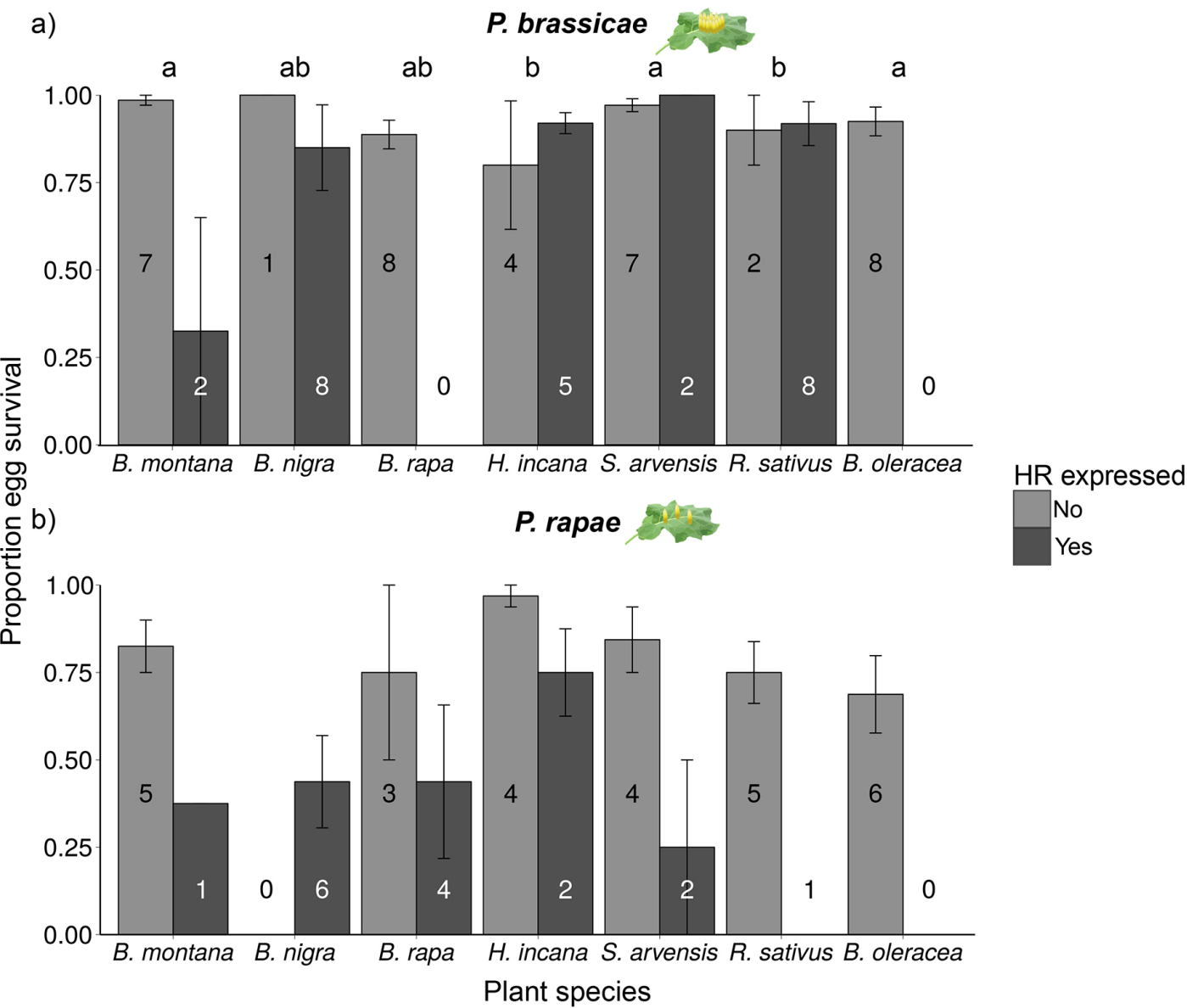

Figure 4.2: Effect of HR-like necrosis on survival of eggs of two Pieris species on different brassicaceous plant species (mean proportion $\pm \mathrm{SE}$ ). Numbers given in the bars indicate the number of plants. Different letters indicate significant differences $(P<0.05$, GLM) between plant species regardless of HR-like necrosis. a) Proportion survival of $P$. brassicae eggs, with each egg clutch consisting of 20 eggs. b) Proportion survival of $P$. rapae eggs, with eight eggs being laid per plant. 
Correlation between oviposition preference and egg survival We did not detect a significant correlation between oviposition preference and survival of the eggs for either of the two butterfly species (proportion of eggs laid related to egg survival; Spearman correlation; for $P$. brassicae: $\mathrm{S}=32,335, \rho=0.10, P=0.44$, for $P$. rapae $: \mathrm{S}=9086.3$, $\rho=0.01, P=0.97$, Figure 4.3a). However, the proportion of $P$. rapae eggs laid was positively correlated with the proportion of plants expressing HR-like necrosis against those eggs ( $\mathrm{S}=4.57, \rho=0.96, P<0.001$, Spearman correlation, Figure 4.3b). For $P$. brassicae, this correlation was not significant $(S=67.72, \rho=0.19, P=0.64$, Spearman correlation, see Figure 4.3b).

\section{Effect of plant species, egg infestation and HR on larval performance Gregarious} species The weight of 7-day-old P. brassicae caterpillars did not vary significantly depending on the plant species they were feeding on $\left(\chi^{2}=12.44, \mathrm{df}=6, P=0.05, \mathrm{LMM}\right.$, Figure 4.4a). However, the plants' response to prior egg deposition significantly affected performance of $P$. brassicae larvae. Seven-day-old larvae developing on plants that previously had received eggs $(\mathrm{EF})$ significantly gained less weight than those on plants that had not received eggs $(\mathrm{F})\left(\chi^{2}=5.27, \mathrm{df}=1, P=0.02\right.$, LMM, Figure 4.4b). Caterpillars feeding on plants without prior egg deposition gained about $5 \%$ more weight. This egg-mediated effect on performance was independent of the plant species (no interactive effect between plant species and egg infestation on larval weight; $\chi^{2}=2.51, \mathrm{df}=$ $6, P=0.87, \mathrm{LMM})$. HR-like necrosis induced by previously laid eggs did not affect the weight of caterpillars $\left(\chi^{2}=0.72, \mathrm{df}=1, P=0.40\right.$, LMM, see Figure $\left.4.4 \mathrm{c}\right)$, and neither did plant species nor did the interaction between plant species and HR-like necrosis $\left(\chi^{2}\right.$ $=5.76, \mathrm{df}=6, P=0.45$ and $\left.\chi^{2}=5.46, \mathrm{df}=4, P=0.24, \mathrm{LMM}\right)$. The weight of 3-day-old caterpillars is presented in the supplementary material (Supplementary Figure 4.A.3).

Solitary species When considering 7-day-old P. rapae caterpillars on both egg-free and previously egg-deposited plants, their weight was not affected by the plant species they were feeding on $\left(\chi^{2}=5.04, \mathrm{df}=6, P=0.54\right.$; LMM, Figure $\left.4.4 \mathrm{a}\right)$. When excluding the occurrence of HR-like necrosis induced by egg deposition, egg infestation preceding larval feeding did not affect larval weight $\left(\chi^{2}=0.001\right.$, df $=1, P=0.97$; LMM, Figure $4.4 \mathrm{~b})$. Neither did the interaction between egg infestation and plant species affect larval weight $\left(\chi^{2}=1.09\right.$, df $=6, P=0.98, \mathrm{LMM}$, Figure $\left.4.4 \mathrm{c}\right)$. Yet, larvae feeding on EF plants expressing an HR-like necrosis were significantly heavier than those feeding on 
a)

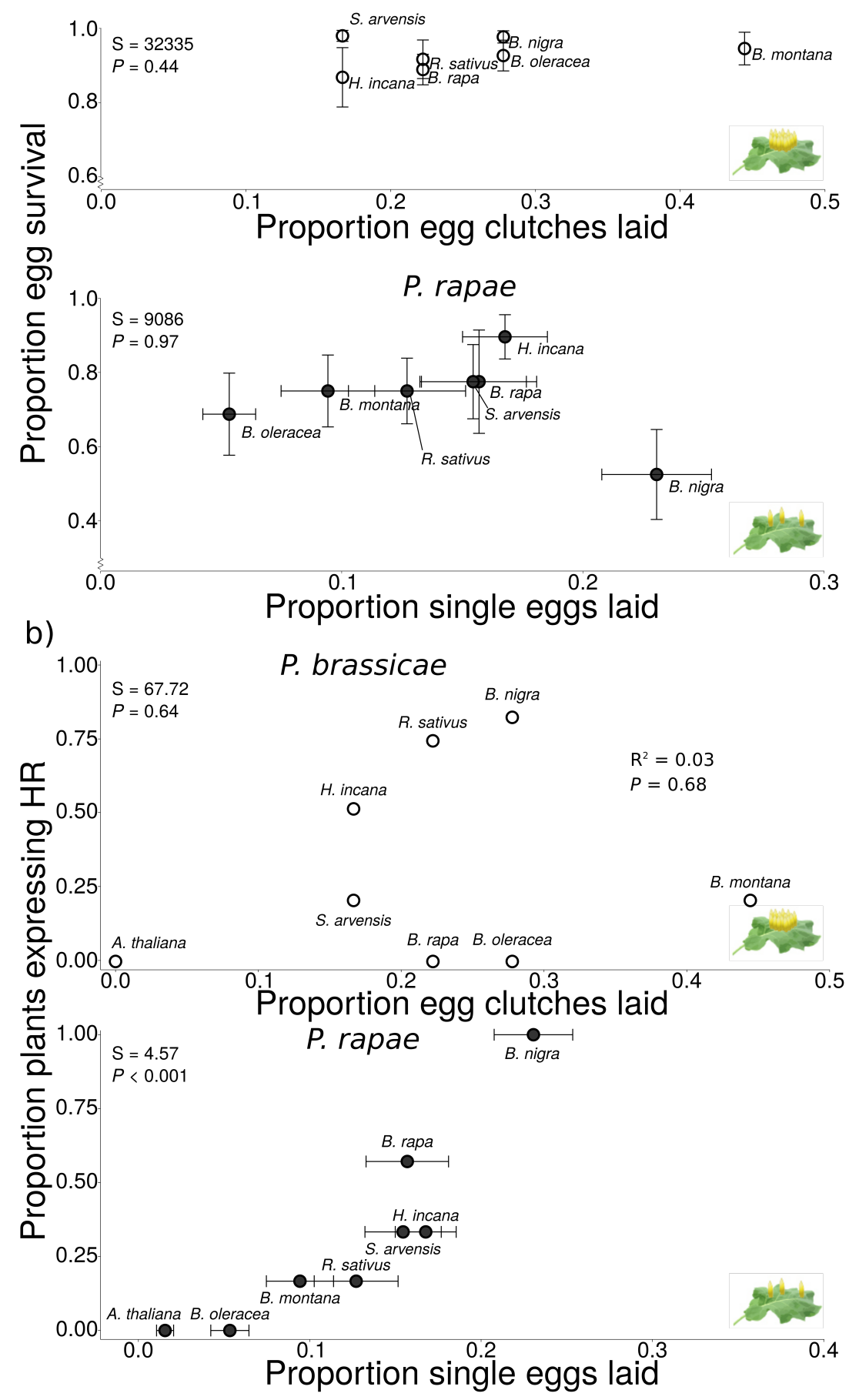

Figure 4.3: Correlation between proportion of eggs laid on different brassicaceous plants by two Pieris butterflies and a proportion of egg survival (mean \pm SE) or b proportion of HR-like necrosis (mean $\pm \mathrm{SE}$ ). Text boxes show correlation results. Proportion of HR+ plants has no error bars, and for $P$. brassicae, no error bars for preference are available. $\mathrm{N}=8-10$ plants for $P$. brassicae, $\mathrm{N}=$ 5-7 plants for $P$. rapae. 
EF plants that did not show HR-like necrosis $\left(\chi^{2}=4.14\right.$, df $=1, P=0.04$, LMM, Figure 4.4c). Neither plant species nor the interaction between plant species and HRlike necrosis affected caterpillar weight on previously egg-infested plants $\left(\chi^{2}=3.73\right.$, df $=6, P=0.71$ and $\chi^{2}=3.93$, df $=3, P=0.27$, LMM). The weight of 3-day-old caterpillars is presented in the supplementary material (Supplementary Figure 4.A.3).

Correlation between oviposition preference and larval performance To assess if there was a correlation between adult oviposition preference and larval performance, we first analysed the relationship between the proportion of eggs laid and the weight of three (Supplementary information and Supplementary Figure 4.A.4) or 7-day-old caterpillars feeding on previously oviposited EF plants and egg-free F plants for each plant species.

Gregarious species Weight of 7-day-old P. brassicae larvae significantly and positively correlated with the number of eggs laid. Seven-day-old $P$. brassicae larvae were the heaviest on those plant species that received most egg clusters $(S=15,964,000, \rho$ $=0.17, P<0.001$, Spearman correlation, Figure $4.5 \mathrm{a}$ ). The proportion of egg clusters laid did not correlate with the weight of caterpillars feeding on egg-free plants $(S=34$, $\rho=0.39, P=0.40$, Spearman correlation, Figure $4.5 \mathrm{~b})$. When considering the weight of 7-day-old $P$. brassicae caterpillars with respect to the plant's capability to express $\mathrm{HR}$ in response to the eggs, weight of caterpillars feeding on previously egg-deposited HR-expressing plants did not correlate with the proportion of egg clutches per plant $(\mathrm{S}=$ $22, \rho=-0.1, P=0.95$, Spearman correlation). Neither was a correlation found between the proportion of plants expressing HR-like necrosis in response to oviposition and the caterpillar weight $(\mathrm{S}=36.65, \rho=0.35, P=0.45$, Spearman correlation).

Solitary species In contrast to $P$. brassicae, the weight of 7-day-old $P$. rapae larvae feeding on previously egg-infested plants neither correlated with the proportion of eggs laid ( $\mathrm{S}=340,680, \rho=0.13, P=0.17$, Spearman correlation, Figure $4.5 \mathrm{c}$ ) nor with egg load when larvae were feeding on egg-free plants $(\mathrm{S}=50, \rho=0.11, P=0.84$, Spearman correlation, Figure 4.5d). Weight of 7-day-old caterpillars feeding on HR+ plants did not correlate with the proportion of eggs laid $(\mathrm{S}=32, \rho=-0.6, P=0.35$, Spearman correlation). Furthermore, the weight of 7-day-old larvae was not correlated with the proportion of plants expressing HR-like necrosis $(\mathrm{S}=29.52, \rho=0.47, P=0.28$, Spearman correlation). 
a)
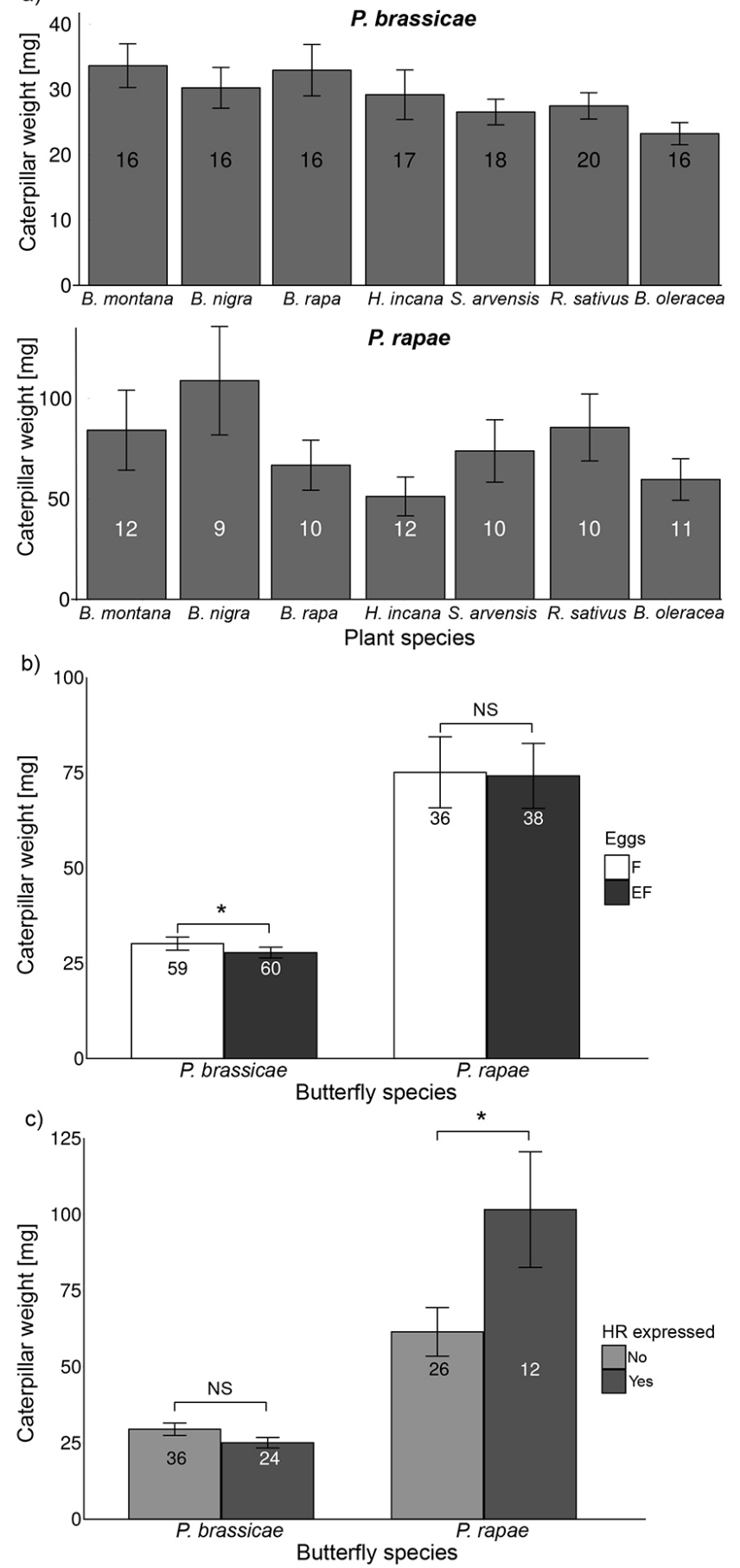

Figure 4.4: Effects of plant species (a), egg-mediated defences (b) or leaf necrosis (c) on weight (mean $\pm \mathrm{SE}$ ) of 7-day-old Pieris brassicae or P. rapae caterpillars. For a weights of caterpillars feeding upon egg-free and previously egg-deposited plants are pooled, b weights of caterpillars feeding upon egg-free (F) and previously egg-deposited plants (EF) are shown separately, and c weights of caterpillars feeding upon previously egg-deposited plants are shown separately by the presence/absence of egg-induced HR-like necrosis. Numbers in the bars represent the number of plants within the group. The weight of caterpillars was averaged per plant. Asterisks indicate significant differences. $* P<0.05$, ns not significant, GLMM. 


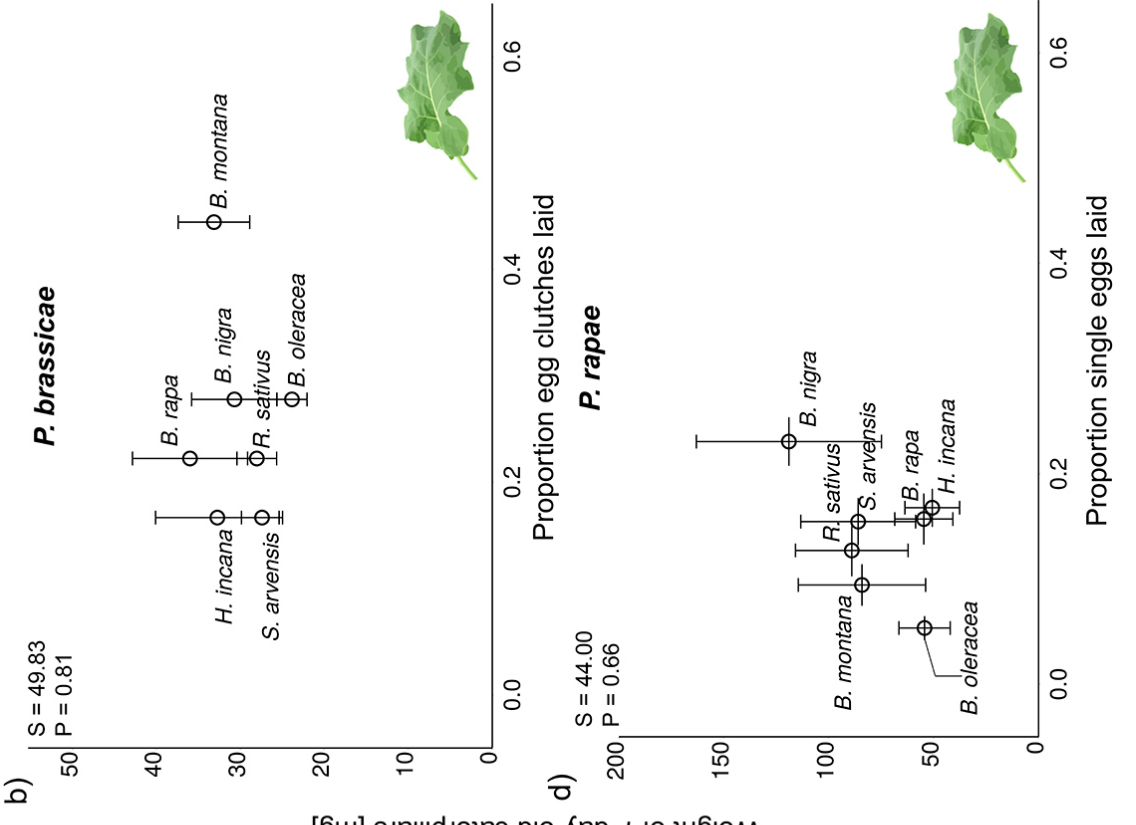

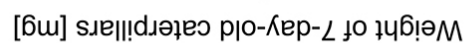

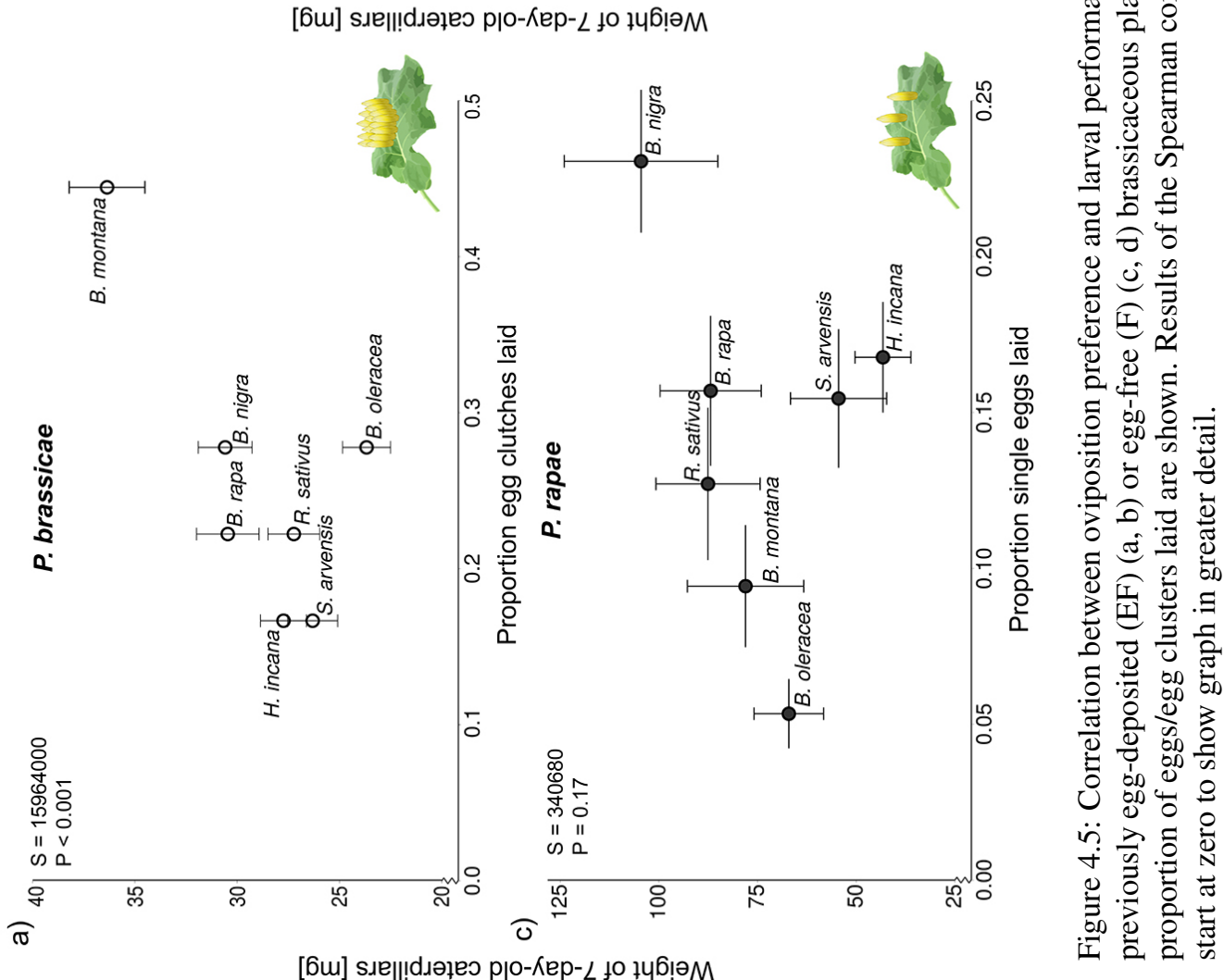




\section{Discussion}

We show that plant-mediated effects of egg deposition partly explain the positive relationship between the proportion of eggs laid and larval weight. Seven-day-old caterpillars of $P$. brassicae gained most biomass on the plant species that received most eggs (B. montana) only when including the effect of previous egg deposition. However, oviposition choices of both Pieris species did not correlate with egg survival. Singly laid $P$. rapae eggs always induced HR-like leaf necrosis in B. nigra on which egg survival was the lowest. Nevertheless, the solitary butterfly deposited most eggs on this plant species. Larval biomass of $P$. rapae was higher on plants expressing egg-induced HR-like necrosis compared to plants without necrosis. In contrast, the gregarious $P$. brassicae showed no significant oviposition preference for any of the tested plant species, and egg survival was hardly affected by HR-like necrosis. However, weight of $P$. brassicae caterpillars feeding on previously egg-deposited plants was lower than of those feeding on egg-free plants.

Females of the solitary species $P$. rapae laid most eggs on B. nigra plants on which egg survival was lowest but this might not be considered an "oviposition mistake" (Larsson and Ekbom, 1995). Oviposition choices are influenced by different cues over long and short distances (Schoonhoven et al., 2005). Cues that signal intraspecific variation in suitability might be absent or of low detectability (Larsson and Ekbom, 1995). Most plants show phenotypic variation in the expression of HR-like necrosis and it might be difficult for the butterflies to discriminate between egg-resistant $(\mathrm{HR}+)$ and eggsusceptible (HR-) genotypes. Yet, a better larval performance might trade-off with a low egg survival. Weight of $P$. rapae caterpillars was highest on $B$. nigra plants expressing HR where egg survival was reduced most. High larval hatching rates on plants with high egg load might result in several problems for the larvae, i.e., fast food depletion, easy detectability of caterpillars by parasitoids, increased cannibalism and the spread of pathogens (Prokopy and Roitberg, 2001).

Similar preference and performance correlations were obtained in studies with the polyphagous Anastrepha ludens fruit fly in response to six different host plants belonging to different families. The second most preferred plant species for oviposition, Casimiroa edulis (white sapote), was also the host on which larvae performed best. However, approximately half of all egg clutches laid on $C$. edulis were killed by a wound tis- 
sue growth response that led to egg encapsulation (Aluja et al., 2004; Birke and Aluja, 2018). We suggest that $P$. rapae butterflies can afford laying most eggs on the most nutritive plants, because a nutritionally high-quality plant can harbor numerous larvae of the solitary species without competing for resources. While survival rate of $P$. rapae eggs on the most preferred host $B$. nigra was lowest, survival rate of $P$. brassicae eggs on the plant species on which most eggs were laid (B. montana) was similar to the plant species that received fewer eggs. This suggests that $P$. brassicae does not adjust its oviposition choices to egg survival rates. P. brassicae can afford to be less choosy when selecting a host plant for oviposition because gregariously laid eggs have certain advantages with regard to egg survival over singly laid eggs. Gregariousness may, for example, contribute to protection from desiccation inflicted by the necrosis (Stamp, 1980; Clark and Faeth, 1998; Griese et al., 2017).

A positive relationship was found between plants expressing HR-like leaf necrosis induced by singly laid $P$. rapae and the oviposition preference of $P$. rapae. No such relationship was found between oviposition choices of $P$. brassicae and the occurrence of HR-like leaf necrosis in the various tested plant species. It is possible that those $P$. rapae larvae hatching from eggs despite HR-like necrosis also face less competition, eventually leading to heavier larvae than on non-HR plants. Another possibility could be that caterpillars perform best on those plant species showing strong HR-like necrosis, because these plants provide high nutritional quality. Based on a meta-analysis, Wetzel et al. (2016) suggested that host plant nutritional quality might be more important for offspring performance than plant defences against larvae. For example, relationships between plant nutrients and performance of noctuid caterpillars (Heliothis virescens and Helicoverpa zea) were consistently concave down, while the relationships between plant defences and herbivore performance were close to linear.

Plant-mediated effects of eggs on caterpillar performance were only shown in the case of the gregarious $P$. brassicae, but not for the solitary $P$. rapae. When comparing weight of caterpillars on egg-free and previously egg-deposited plants (all species), P. brassicae caterpillars gained less weight on the latter. These results confirm previous results on the performance of $P$. brassicae tested on A. thaliana (Geiselhardt et al., 2013), B. nigra (Bonnet et al., 2017; Pashalidou et al., 2013; Pashalidou et al., 2015a; Pashalidou et al., 2015b) or S. arvensis and B. oleracea (Pashalidou et al., 2015a). Similarly, reinforced plant defence against insect larvae mediated by prior egg deposition occurs in several 
interactions between plants and insects that lay eggs in clutches and feed gregariously during larval development, namely Diprion pini sawflies on pine (Beyaert et al., 2012); Spodoptera littoralis caterpillars on wild tobacco, (Bandoly et al., 2015; Bandoly et al., 2016) and Xanthogaleruca luteola leaf beetles on elm (Austel et al., 2016). Singly laid eggs of $P$. rapae did not prime plant defence against caterpillars. The reason why only plant responses to egg clutches of the gregarious $P$. brassicae negatively affected the performance of subsequently feeding caterpillars and not the singly laid eggs of $P$. rapae remains to be investigated.

The differences in preference and performance recorded for the two butterfly species might be due to their different oviposition and feeding modes. Unlike for singly laid eggs ( $P$. rapae), survival of clustered egg (P. brassicae) was not lower on plants expressing HR-like necrosis. This confirms a previous study where survival of $P$. brassicae eggs on B. nigra expressing HR-like necrosis was not affected even under field conditions (Griese et al., 2017). However, when P. brassicae eggs were experimentally kept singly, mortality increased. Single eggs suffered more from drops in humidity than a cluster of five eggs (Griese et al., 2017). Humidity drops are likely a characteristic of necrotic leaf tissue and a possible reason for egg-killing (Shapiro and DeVay, 1987). Hence, our current study supports the assumption that egg-induced HR-like necrosis negatively affects survival of singly laid eggs (as those of $P$. rapae) rather than clustered eggs (as those of $P$. brassicae). This is further supported by previous studies which showed that single eggs of $P$. rapae as well as of $P$. napi suffer high mortality when the host plant expresses HR-like necrosis (Shapiro and DeVay, 1987; Fatouros et al., 2014).

With respect to our initial questions, our study has shown that $P$. brassicae laid most eggs on those plant species which provide the best larval performance, while these plant species do not provide best egg survival rates. The plant species' capability to activate HR-like necrosis in response to egg deposition affected the oviposition choice of $P$. rapae in so far, as this butterfly species laid most eggs on a plant species where HR expression frequently occurred, and egg survival rates were low. This behavior might be considered a counter-adaptation because a high egg load on a plant with a high eggkilling capability ensures survival of at least some offspring. Since survival of eggs of $P$. brassicae was not affected by the plant's HR-like, this butterfly species can afford to lay many eggs on a plant species with high HR-expression frequency. Our data indicate that the gregarious oviposition mode of $P$. brassicae allows this butterfly species to be 
less choosy than $P$. rapae in selecting an oviposition site because the gregariously laid eggs are not affected by HR.

Future studies need to further address the question whether the differences in the effects of plant responses to these two Pierid species are due to the different oviposition modes and larval feeding behaviors (singly vs. gregariously) or whether other insect species-specific traits are important as well. Moreover, our results on positive oviposition preference-larval performance relationships and effects of the egg phase therein were mainly driven by two plant species. Therefore, we suggest to further test the PPH with a different or more numerous set of plant species to gain a better understanding of how general these effects are. Previous studies have shown that egg-mediated priming affects several performance traits apart from larval weight, i.e., developmental time, pupal weight and larval survival (Pashalidou et al., 2015a; Hilker and Fatouros, 2015; Hilker and Fatouros, 2016). Yet, it remains to be investigated whether oviposition preference correlates with these other performance measures. Furthermore, future studies need to explore whether clustering of eggs by $P$. brassicae and a high number of singly laid eggs for $P$. rapae may evolved as counter adaptations against the egg-killing leaf necrosis. Other countermeasures of pierid butterflies against egg-induced plant defences include oviposition on inflorescence stems instead of leaves, as occurs in some other pierid species feeding on Brassicaceae (P. napi and Anthocharis cardamines) (Griese et al., 2021) and feeding preference for flowers when they are available (A. cardamines and P. brassicae) (Wiklund and Åhrberg, 1978; Smallegange et al., 2007).

\section{Material and methods}

Insects and plants Pieris brassicae L. and P. rapae L. (Lepidoptera: Pieridae) were reared since many generations in a climatised room $\left(21 \pm 1{ }^{\circ} \mathrm{C}, 50-70 \% \mathrm{RH}, \mathrm{LD} 16: 8\right)$ on B. oleracea var. gemmifera L. plants. Female butterflies mated 2-3 days after eclosion. Their oviposition preference was tested 2 days after mating. The females have a high egg load at this age and mating status (David and Gardiner, 1962).

Eight different brassicaceous species were used in a preference experiment, seven in a performance experiment. Apart from Raphanus sativus L., all plant species were non-domesticated species. We obtained $R$. sativus from De Bolster seed company (The Netherlands), Hirschfeldia incana L. Lagr.-Foss. from the US, California, Brassica 
nigra L. from the Centre of Genetic Resources (CGN, Wageningen, the Netherlands) from an early flowering accession (CGN06619), Sinapis arvensis L. from Vlieland, in the north of The Netherlands, B. montana Pourr. from CGN (CGN18472 accession from Italy), B. rapa L. from Binnenveld, west of Wageningen (The Netherlands) and B. oleracea L. 'Kimmeridge' from the south coast of England. Most of the tested plant species are frequently used as host plants by both species in the Netherlands, especially B. nigra, B. rapa, B. oleracea and S. arvensis, except for A. thaliana (Fei et al., 2014; Harvey et al., 2007). Natural accessions of $A$. thaliana are visited by pierids in the North of Europe (Wiklund, 1984). Hirschfeldia incana was shown to be a host plant for $P$. rapae in California (Shapiro, 2002). Brassica montana is a wild ancestor of B. oleracea; both species grow in coastal regions either in Southern Europe or Britain, respectively. Arabidopsis thaliana (Col-0) was used only for the preference tests. Because of its small size, it was excluded from performance studies, as more than just the focal egg-induced plant would be needed to feed the caterpillars. All plants were in the non-flowering stage when tested, except A. thaliana, which already flowered. All plants were cultivated in pots filled with standard potting soil and watered daily; no fertilization was added. They were grown in a climate room $\left(18 \pm 4{ }^{\circ} \mathrm{C}, 60-80 \% \mathrm{RH}, \mathrm{LD} 16: 8\right)$. To use plants of similar biomass in the bioassays, B. oleracea was 4 weeks old, and all other plants were 3 weeks old at the time of infestation.

Butterfly oviposition preference To determine which plant species is preferred for oviposition, we simultaneously offered the above-mentioned eight plant species to a mated female butterfly. An individual plant of each species was placed into a mesh cage $(75 \times 75 \times 115 \mathrm{~cm})$. The plants were set up in a circle with the leaves not touching each other. The design was a randomized block with 18 replicates per butterfly species. The two butterfly species were tested in separate cages at different time points in a greenhouse compartment $\left(23 \pm 5^{\circ} \mathrm{C}, 50-70 \% \mathrm{RH}, \mathrm{LD} 16: 8\right)$. After placing the plants inside a cage, one mated female butterfly was released. The number of $P$. rapae eggs or $P$. brassicae egg clutches, respectively, was counted on each plant $3 \mathrm{~h}$ after release of the butterfly. Preliminary experiments showed that most butterfly females will make an oviposition choice within this time period. 
Plant treatments for performance tests To assess egg survival rates and performance of larvae on previously egg-deposited plants, a plant individual of each species was infested with either P. rapae or P. brassicae eggs. Each plant was placed in a cage with approximately 100 butterflies, located in a climate room $\left(21 \pm 1{ }^{\circ} \mathrm{C}, 50-70 \% \mathrm{RH}, \mathrm{LD}\right.$ 16:8). The first fully developed leaf of each plant (fourth or fifth from the top) was exposed to either $P$. brassicae or $P$. rapae butterflies for egg deposition, while the rest of the plant was covered with a fine mesh. We limited the number of eggs deposited onto a plant to 20 eggs of $P$. brassicae (laid in a clutch) and to eight single $P$. rapae eggs per plant. Limiting egg deposition was done by observing the butterflies after introduction into the cages and removing them as soon as they had deposited the mentioned number of eggs. Those numbers were chosen to mimic naturally occurring egg numbers per plant (Feltwell, 1982; Fatouros et al., 2014). Occasionally, extra laid eggs were immediately removed using a fine brush [see Pashalidou et al. (2013) for details]. In total, seven to nine individual plants per species were infested with $P$. brassicae eggs, and six to seven individual plants per species received $P$. rapae eggs.

Plant response to egg deposition, egg mortality and larval performance To determine egg survival, we counted the number of larvae hatching from the twenty $(P$. brassicae) or eight (P. rapae) eggs deposited on a plant. After 4 days, presence/absence of HR-like necrosis was scored as previously described by Griese et al. (2017). After 5 days, survival of eggs was noted by counting the number of hatched caterpillars. To assess larval performance and the impact of the plant's response to previous egg deposition on larval performance, we divided the neonate larvae hatching from egg-deposited plants into two groups. Half of them were placed back onto the previously egg-infested plant (labeled 'egg and feeding', EF) (on the adaxial side of the leaf where they hatched), and the other half was transferred to an egg-free plant (labeled 'feeding', F) plant of the same species and placed onto the adaxial side of the leaf as well. Three and seven days after hatching, caterpillar weight was measured on a microbalance (accuracy $=1 \mu \mathrm{g}$; Sartorius AG, Göttingen, Germany). We weighed each caterpillar individually, and afterwards the caterpillars were transferred back to their original position, on EF or F plants. Every EF and F plant was considered one replicate. 
Statistical analysis Data on $P$. rapae oviposition preference for host plants were analysed by a generalized linear model (GLM) (Poisson distribution), with plant species as fixed factor and the number of eggs per plant as response variable. The post hoc analysis was performed using a linear hypothesis test (multcomp package). Because $P$. brassicae laid most eggs in a single clutch each time, only oviposition 'yes' or 'no' was scored when determining oviposition preference. These data were analysed by a GLM (binomial distribution) with the plant species as fixed factor, and the presence/absence of oviposition as response variable (post hoc test: linear hypothesis test).

Data on egg survival of each butterfly species were analysed by a generalized linear mixed effect model (GLMM, lme4 package) with binomial distribution. The model included egg survival as response variable, and plant species, presence/absence of HR as well as the interaction between both variables were fixed factors. Date of infestation were a random factor. A post hoc analysis was conducted using linear hypothesis tests for plant species and interaction terms.

To evaluate whether oviposition preference for a plant species can be linked to egg survival, we ran a correlation analysis using Spearman correlation as well as linear regression to generate regression lines. We conducted this analysis first by relating the proportion of eggs (or egg clutches) laid on each plant species with the proportion of eggs surviving on each plant species. To elucidate the relationship between the plant's ability to express HR-like necrosis and egg survival, we correlated the proportion of eggs laid on each plant species to the proportion of eggs surviving on those plants, which expressed HR-like necrosis in response to the eggs.

To elucidate the relationship between the plants' ability to express HR-like necrosis and oviposition preference, we linked the proportion of eggs or egg clusters laid to the proportion of plants expressing HR.

Data on caterpillar weight obtained on all plant species (subjected to prior egg deposition or not) were analysed using linear mixed effect models (LMM). We calculated the average caterpillar weight per plant. The logarithm of the mean caterpillar weight 3 or 7 days after hatching was the dependent variable, plant species and egg infestation as well as the interaction between them were used as independent variables, the random factor was the date of egg infestation.

A post hoc analysis was conducted using linear hypothesis tests for plant species and interaction terms. The effect of HR-like necrosis on caterpillar weight was tested using 
the subset of plants infested with eggs and performing LMM on the logarithmic data of the mean caterpillar weight. Expression of HR-like necrosis, plant species as well as the interaction between both factors was included in the model. A post hoc analysis was conducted using linear hypothesis tests for plant species and interaction terms.

To detect possible links between butterfly oviposition preference and performance of 3- or 7-day-old caterpillars, a linear regression analysis was conducted. We conducted this analysis first by relating the proportion of eggs laid on each plant species with the weight of caterpillars on each plant species. Furthermore, we ran an analysis by relating the proportion of eggs laid on each plant species to the weight of caterpillars on (1) plants which received eggs prior to larval feeding (EF) and (2) plants which were left without any eggs (F); thus, we aimed to test the hypothesis that females prefer to oviposit on plants with most modest (for P. rapae : putative) egg-mediated reinforcement of defence against the larvae. In addition, we analysed the relationship between caterpillar weight and the proportion of eggs laid on plants expressing HR-like necrosis; thus, we aimed to test the hypothesis that females prefer to oviposit on plants whose HR-like necrosis has the most modest effect on the performance of their offspring.

Finally, we tested the relationship between the proportion of plants expressing HRlike necrosis and caterpillar weight. Thus, we aimed to gain insight into whether the frequency of HR-like necrosis in response to the eggs relates to caterpillar weight. All analyses were performed using R 3.3.2 (R Core Team, 2016).

\section{Acknowledgements}

We thank André Gidding, Léon Westerd, Joop Woelke and Frans van Aggelen for culturing insects and Unifarm of Wageningen University for providing plants and Rieta Gols for providing seeds used in the experiments. This work was supported by the German Academic Exchange Service (DAAD) (57044990), the German Research Foundation (DFG, CRC 973; www.sfb973.de) and the Dutch Research Council (NWO) (NWO/ALW Veni grant no. 863.09.002 and NWO/TTW Vidi grant no. 14854). 


\section{A Supplementary material}

\section{Results}

Effect of plant species, egg infestation and HR on performance of three-day-old larvae, gregarious species. Weight of three-day-old $P$. brassicae caterpillars varied in dependence of the plant species they were feeding upon $\left(\chi^{2}=28.70\right.$, $\mathrm{df}=6, P<0.001$, LMM, Figure 4.A.3A). However, the post hoc analysis did not show significant differences in weights of larvae feeding upon the various plant species. Neither did infestation of plants with eggs prior to larval feeding $\left(\chi^{2}=0.03\right.$, df $\left.=1, P=0.87, \mathrm{LMM}\right)$ nor the interaction between the factors 'egg infestation' and 'plant species' $\left(\chi^{2}=0.95, \mathrm{df}=6, P=\right.$ 0.99 , LMM) affect weight of larvae three days after hatching (Figure 4.A.3B). Furthermore, we tested whether HR-like necrosis induced by the egg deposition affects larval weight by considering only plants that previously received eggs (EF). No significant weight differences were found between caterpillars that fed on HR- or HR+ plants $\left(\chi^{2}\right.$ $=0.04, \mathrm{df}=1, P=0.84, \mathrm{LMM}$, Figure $4.5 \mathrm{c}$ ). Again, weights of larvae feeding on the tested plant species varied significantly $\left(\chi^{2}=18.09, \mathrm{df}=6, P=0.006, \mathrm{LMM}\right)$, but the linear hypothesis (post hoc) test did not show differences in weights of larvae feeding upon certain plant species. There was no significant interaction between the effects of 'plant species' and 'HR' on larval weight $\left(\chi^{2}=5.28, \mathrm{df}=4, P=0.26, \mathrm{LMM}\right)$.

Solitary species. Weight of three-day-old $P$. rapae caterpillars was neither affected by plant species they were feeding upon, nor by egg deposition prior to larval feeding nor by the interaction between plant species and egg infestation $\left(\chi^{2}=5.31\right.$, df $=6, P=$ $0.50 ; \chi^{2}=0.28, \mathrm{df}=1, P=0.60$ and $\chi^{2}=0.16, \mathrm{df}=6, P=1.00$ respectively, LMM Figure 4.A.3A and B). However, the HR-like necrosis induced by egg deposition prior to larval feeding significantly affected larval performance. Larvae feeding on HR+ plants were significantly heavier than HR-plants $\left(\chi^{2}=8.12\right.$, df $=1, P=0.004$, LMM, Figure 4.A.3C). Plant species and interaction between plant species and HR- like expression did not affect caterpillar weight on plants previously exposed to eggs (EF) $\left(\chi^{2}=7.44\right.$, $\mathrm{df}=6, P=0.28$ and $\left.\chi^{2}=0.35, \mathrm{df}=3, P=0.95, \mathrm{LMM}\right)$. 
Chapter 4 Egg induced responses and PPH

Table 4.A.1: Results of the post hoc test on oviposition preference of $P$. brassicae and $P$. rapae differences between species.

\begin{tabular}{|c|c|c|c|}
\hline Butterfly species & Species comparison & $\mathrm{z}$ value & $P$ value \\
\hline \multirow[t]{25}{*}{ P. brassicae } & B. montana - A. thaliana & 0.01 & 1.00 \\
\hline & B. nigra - A. thaliana & 0.01 & 1.00 \\
\hline & B. oleracea - A. thaliana & 0.01 & 1.00 \\
\hline & B. rapa - A. thaliana & 0.01 & 1.00 \\
\hline & H. incana - A. thaliana & 0.01 & 1.00 \\
\hline & R. sativus - A. thaliana & 0.01 & 1.00 \\
\hline & S. arvensis - A. thaliana & 0.01 & 1.00 \\
\hline & B. nigra - B. montana & -1.03 & 0.96 \\
\hline & B. oleracea - B. montana & -1.03 & 0.96 \\
\hline & B. rapa - B. montana & -1.39 & 0.84 \\
\hline & H. incana - B. montana & -1.75 & 0.61 \\
\hline & R. sativus - B. montana & -1.39 & 0.84 \\
\hline & S. arvensis - B. montana & -1.75 & 0.61 \\
\hline & B. oleracea - B. nigra & 0.00 & 1.00 \\
\hline & B. rapa - B. nigra & -0.38 & 1.00 \\
\hline & H. incana - B. nigra & -0.80 & 0.99 \\
\hline & R. sativus - B. nigra & -0.38 & 1.00 \\
\hline & S. arvensis - B. nigra & -0.80 & 0.99 \\
\hline & B. rapa - B. oleracea & -0.38 & 1.00 \\
\hline & H. incana - B. oleracea & -0.80 & 0.99 \\
\hline & R. sativus - B. oleracea & -0.38 & 1.00 \\
\hline & S. arvensis - B. oleracea & -0.80 & 0.99 \\
\hline & H. incana - B. rapa & -0.42 & 1.00 \\
\hline & R. sativus - B. rapa & 0.00 & 1.00 \\
\hline & S. arvensis - B. rapa & -0.42 & 1.00 \\
\hline
\end{tabular}




\begin{tabular}{|c|c|c|c|}
\hline \multicolumn{4}{|c|}{ continued from previous page } \\
\hline Butterfly species & Species comparison & $\mathrm{z}$ value & $P$ value \\
\hline \multirow[t]{3}{*}{ P. brassicae } & R. sativus - H. incana & 0.42 & 1.00 \\
\hline & S. arvensis - H. incana & 0.00 & 1.00 \\
\hline & S. arvensis - R. sativus & -0.42 & 1.00 \\
\hline \multirow[t]{24}{*}{ P. rapae } & B. montana - A. thaliana & 6.67 & $<0.001$ \\
\hline & B. nigra - A. thaliana & 10.42 & $<0.001$ \\
\hline & B. oleracea - A. thaliana & 4.52 & $<0.001$ \\
\hline & B. rapa - A. thaliana & 8.48 & $<0.001$ \\
\hline & H. incana - A. thaliana & 8.87 & $<0.001$ \\
\hline & R. sativus - A. thaliana & 7.17 & $<0.001$ \\
\hline & S. arvensis - A. thaliana & 8.48 & $<0.001$ \\
\hline & B. nigra - B. montana & 7.19 & $<0.001$ \\
\hline & B. oleracea - B. montana & -3.08 & 0.04 \\
\hline & B. rapa - B. montana & 3.22 & 0.03 \\
\hline & H. incana - B. montana & 3.98 & 0.002 \\
\hline & R. sativus - B. montana & 0.83 & 0.99 \\
\hline & S. arvensis - B. montana & 3.22 & 0.02 \\
\hline & B. oleracea - B. nigra & -9.42 & $<0.001$ \\
\hline & B. rapa - B. nigra & -4.26 & $<0.001$ \\
\hline & H. incana - B. nigra & -3.50 & 0.01 \\
\hline & R. sativus - B. nigra & -6.49 & $<0.001$ \\
\hline & S. arvensis - B. nigra & -4.26 & $<0.001$ \\
\hline & B. rapa - B. oleracea & 6.02 & $<0.001$ \\
\hline & H. incana - B. oleracea & 6.69 & $<0.001$ \\
\hline & R. sativus - B. oleracea & 3.86 & 0.002 \\
\hline & S. arvensis - B. oleracea & 6.02 & $<0.001$ \\
\hline & H. incana - B. rapa & 0.79 & 0.99 \\
\hline & R. sativus - B. rapa & -2.41 & 0.22 \\
\hline
\end{tabular}

continued on next page 
Chapter 4 Egg induced responses and $\mathrm{PPH}$

\begin{tabular}{llll}
\hline \multicolumn{2}{l}{ continued from previous page } \\
\hline Butterfly species & Species comparison & z value & $P$ value \\
\hline \multirow{2}{*}{. rapae } & S. arvensis - B. rapa & 0.00 & 1.00 \\
& R. sativus - H. incana & -3.19 & 0.03 \\
& S. arvensis - H. incana & -0.79 & 0.99 \\
& S. arvensis - R. sativus & 2.41 & 0.22 \\
\hline
\end{tabular}

Table 4.A.2: Number of infested plants and percentage of these plants expressing HR-like necrosis for both butterfly species separated for all plant species.

\begin{tabular}{lllll}
\hline & \multicolumn{2}{c}{ P. brassicae } & \multicolumn{2}{c}{ P. rapae } \\
\hline Plant species & No. infested & $\%$ HR yes & No. infested & $\%$ HR yes \\
B. montana & 9 & 22.22 & 6 & 16.67 \\
B. nigra & 9 & 88.89 & 6 & 100.00 \\
B. oleracea & 8 & 0.00 & 6 & 0.00 \\
B. rapa & 8 & 0.00 & 7 & 57.14 \\
H. incana & 9 & 55.56 & 6 & 33.33 \\
R. sativus & 10 & 80.00 & 6 & 16.67 \\
S. arvensis & 9 & 22.22 & 6 & 33.33 \\
\hline
\end{tabular}


Table 4.A.3: Results of post hoc test on egg survival of $P$. brassicae when compared between plant species.

\begin{tabular}{lll}
\hline Species comparison & $\mathrm{z}$ value & $P$ \\
\hline B. nigra - B. montana & 0.002 & 1.00 \\
B. oleracea - B. montana & -1.11 & 0.90 \\
B. rapa - B. montana & -1.87 & 0.43 \\
H. incana - B. montana & -4.22 & $<0.001$ \\
R. sativus - B. montana & -3.82 & 0.002 \\
S. arvensis - B. montana & -0.90 & 0.96 \\
B. oleracea - B. nigra & -0.002 & 1.00 \\
B. rapa - B. nigra & -0.002 & 1.00 \\
H. incana - B. nigra & -0.002 & 1.00 \\
R. sativus - B. nigra & -0.003 & 1.00 \\
S. arvensis - B. nigra & -0.002 & 1.00 \\
B. rapa - B. oleracea & -1.40 & 0.75 \\
H. incana - B. oleracea & -4.68 & $<0.001$ \\
R. sativus - B. oleracea & -3.60 & 0.004 \\
S. arvensis - B. oleracea & -0.01 & 1.00 \\
H. incana - B. rapa & -3.69 & 0.003 \\
R. sativus - B. rapa & -3.08 & 0.02 \\
S. arvensis - B. rapa & 0.79 & 0.98 \\
R. sativus - H. incana & -1.09 & 0.91 \\
S. arvensis - H. incana & 3.52 & 0.01 \\
S. arvensis - R. sativus & 3.26 & 0.01 \\
\hline
\end{tabular}




\section{Chapter 4 Egg induced responses and $P P H$}

Table 4.A.4: Results of single GLMs for each plant species tested for the influence of HR expression separated for $P$. brassicae on egg survival, used as a post hoc test. The level of significance was 0.05. Dashes were used to indicate that the test was not applicable, because the plant species never expressed HR when exposed to $P$. brassicae eggs.

\begin{tabular}{llll}
\hline & \multicolumn{3}{c}{ P. brassicae } \\
\hline Plant species & $\chi^{2}$ & df & $P$ \\
\hline B. montana & 19.50 & 1 & $<0.001$ \\
B. nigra & 0.32 & 1 & 0.57 \\
B. rapa & - & - & - \\
H. incana & 0.70 & 1 & 0.40 \\
S. arvensis & 1.38 & 1 & 0.24 \\
R. sativus & 0.02 & 1 & 0.89 \\
B. oleracea & - & - & - \\
A. thaliana & - & - & - \\
\hline
\end{tabular}

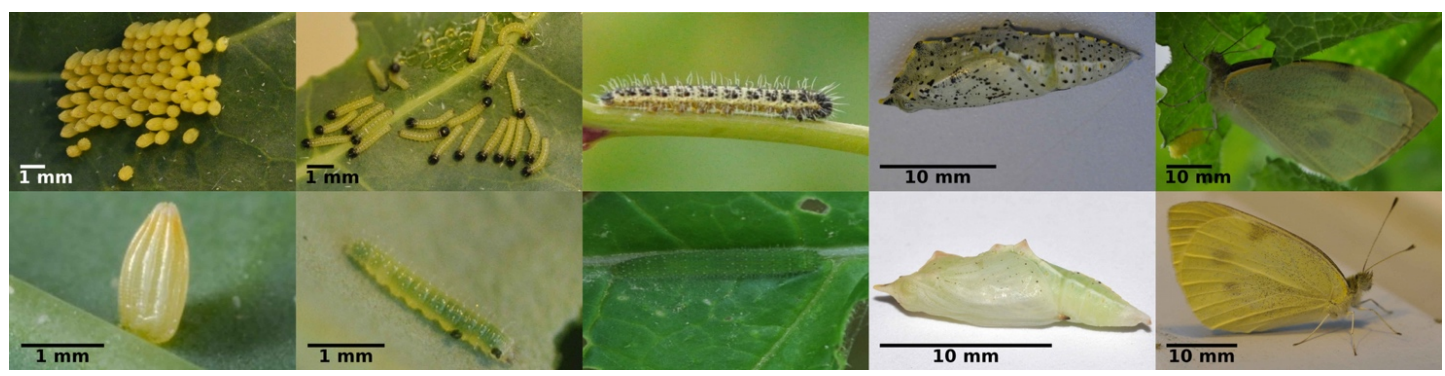

Figure 4.A.1: Developmental stages of the studied Pieris species. Differences in appearance and life history of the gregarious $P$. brassicae (top) and solitary P. rapae (bottom). From left to right: Eggs, neonates, L5 caterpillars, pupae, adults. Pieris brassicae larvae are feeding gregariously until the third larval stage. While eggs and adults of both species look similar and mainly differ in size, caterpillars of the two species are differently coloured. Larvae of $P$. brassicae larvae are aposematically colored (an indication for unpalatability), which allows them to feed in groups, whereas $P$. rapae larvae are cryptically coloured, a trait which is often linked with a solitary feeding behaviour in butterflies (Sillén-Tullberg, 1988). Photo credits: E. Griese. 


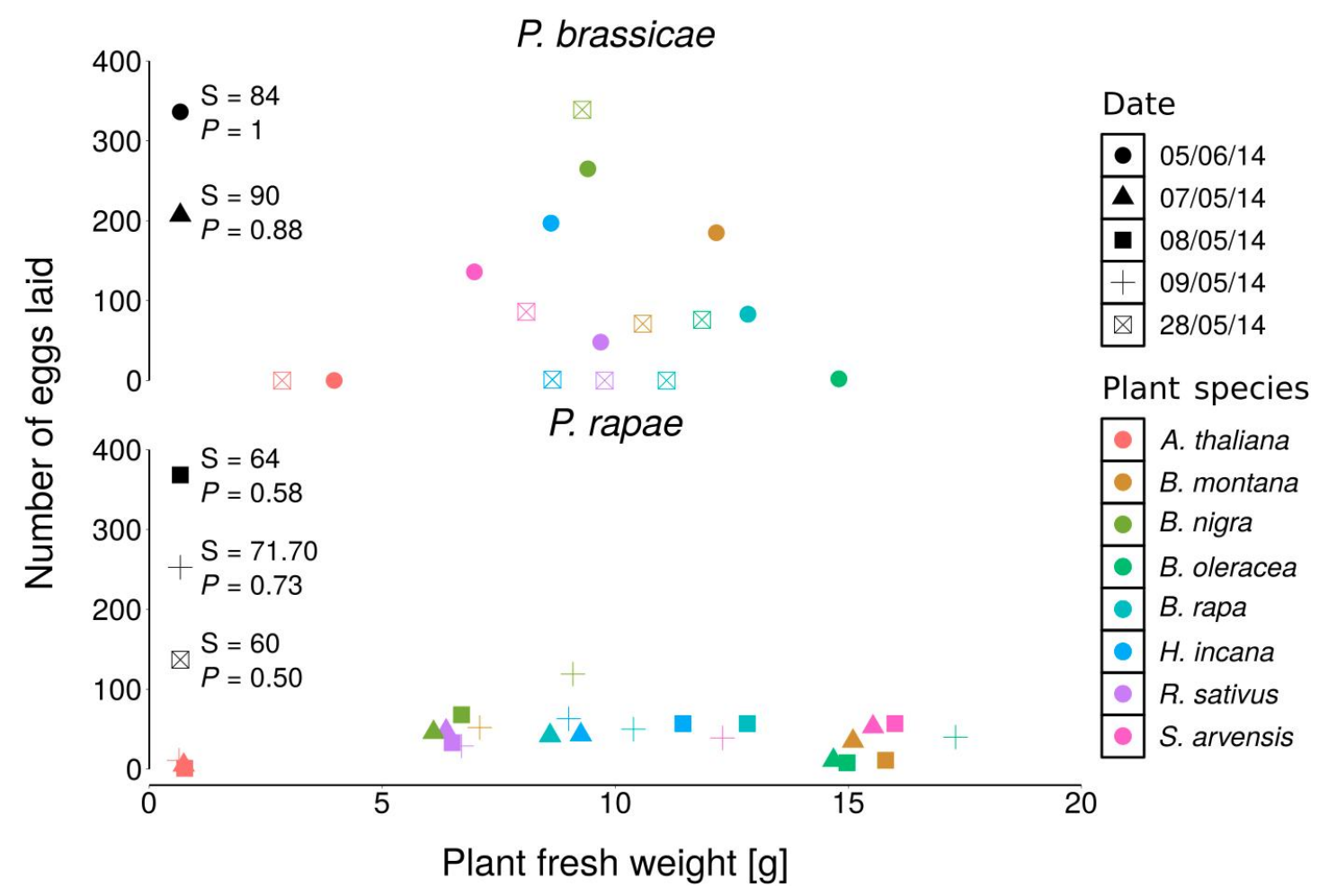

Figure 4.A.2: Correlation between the number of eggs laid and plant fresh weight. Neither for $P$. brassicae nor for $P$. rapae there is a significant correlation between the two factors. Spearman correlation was tested for each date independent of each other. 
Chapter 4 Egg induced responses and $\mathrm{PPH}$

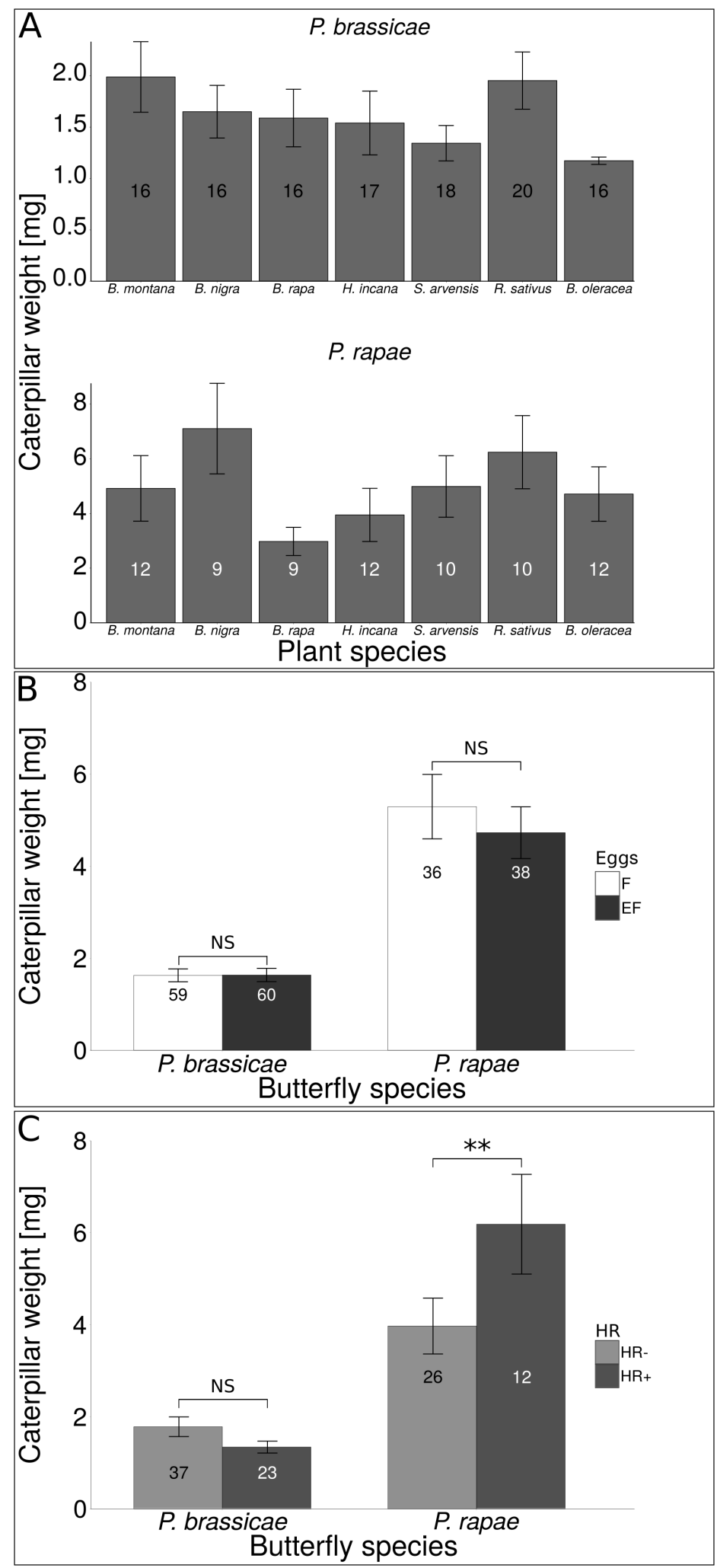

Figure 4.A.3: Caption on next page. 


\section{A Supplementary material}

Figure 4.A.3: (Previous page.) Effect of brassicaceous plant species (a), egg-mediated plant effects, Plant species (b), and HR-like necrosis (c) on weight (mean \pm SE) of three-day-old Pieris caterpillars. In (a), weights of caterpillars feeding upon egg-free and previously egg-deposited plants are pooled. In (b), weights of caterpillars feeding upon egg-free and previously egg-deposited plants are shown separately. In (c), weights of caterpillars feeding upon previously egg-deposited plants are shown separately for plants expressing HR-like necrosis or not in response to the egg deposition. The numbers in the bars represent the number of plants within the group. The weight of caterpillars was averaged per plant. Asterisks indicate significant differences. $* * P<$ 0.01 , ns: not significant, GLMM.

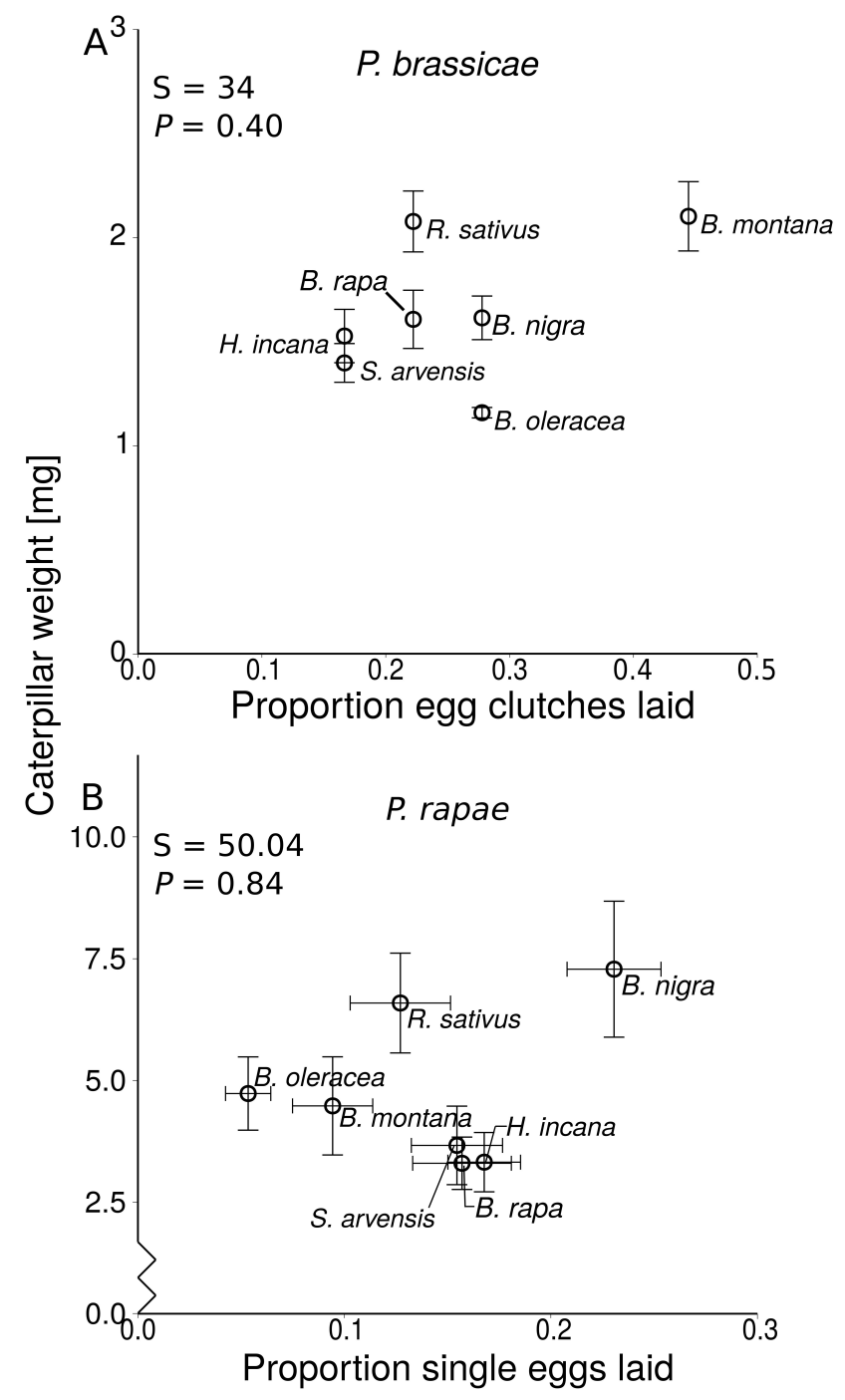

Figure 4.A.4: Linear regression between the proportion of eggs laid and the mass of 3-day old caterpillars (mean $\pm \mathrm{SE}$ ) on egg-infested (EF) plant species. (a) Pieris brassicae correlation, the proportion of egg clutches laid per 18 test plants were used as preference measurement. (b) Pieris rapae correlation mean eggs laid per plant species $( \pm \mathrm{SE})$ were used as preference data. 


\section{Chapter 5}

\section{Insect egg-killing: A new front on the evolutionary arms race between brassicaceous plants and pierid butterflies}

Eddie Griese, Lotte Caarls, Niccolò Bassetti, Setareh Mohammadin, Patrick Verbaarschot, Gabriella Bukovinszkine'Kiss, Erik H. Poelman, M. Eric Schranz \& Nina E. Fatouros

Published in a slightly different version in: New Phytologist (2021), 230, pp. 341-353

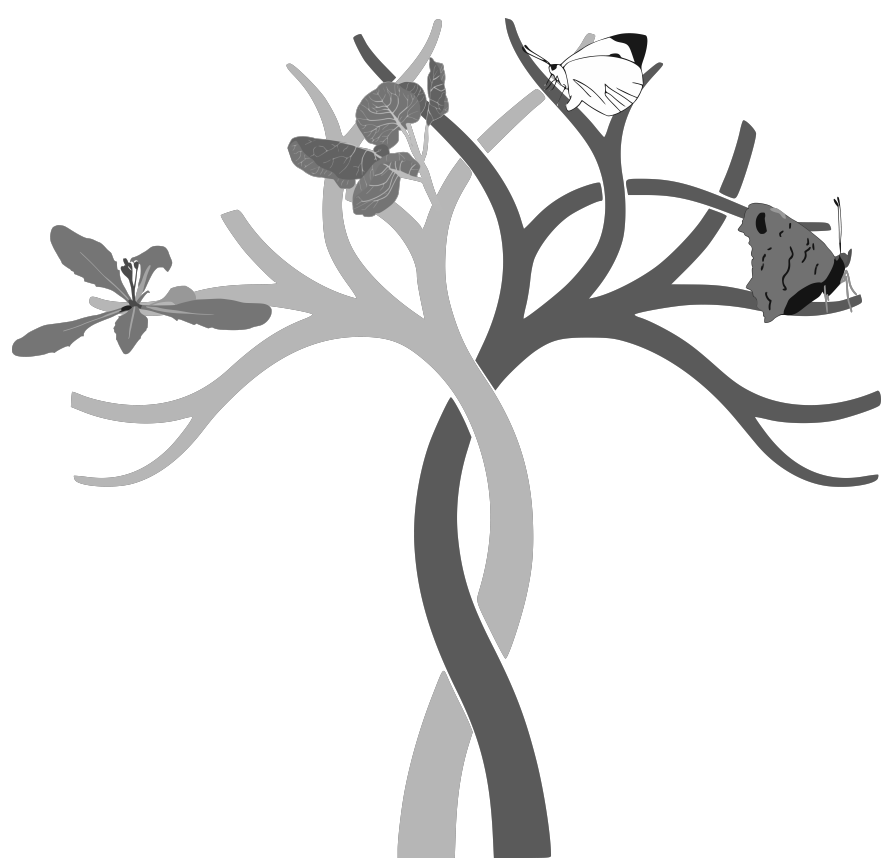




\section{0}

\section{Abstract}

Evolutionary arms races between plants and insect herbivores have long been proposed to generate key innovations such as plant toxins and detoxification mechanisms that can drive diversification of the interacting species. A novel front-line of plant defence is the killing of herbivorous insect eggs. We test whether an egg-killing plant trait has an evolutionary basis in such a plant-insect arms race. Within the crucifer family (Brassicaceae), some species express a hypersensitive response (HR)-like necrosis underneath of butterfly eggs (Pieridae) that leads to eggs desiccating or falling off. We studied the phylogenetic distribution of this trait, its egg-killing effect on and elicitation by butterflies, by screening 31 Brassicaceae species, and nine Pieridae species. We show a clade-specific induction of strong, egg-killing HR-like necrosis mainly in species of the Brassiceae tribe including Brassica crops and close relatives. The necrosis is strongly elicited by pierid butterflies that are specialists of crucifers. Furthermore, HR-like necrosis is linked to PRl defence gene expression, accumulation of reactive oxygen species and cell death, eventually leading to egg killing. Our findings suggest that the plants' egg-killing trait is a new front on the evolutionary arms race between Brassicaceae and pierid butterflies beyond the well-studied plant toxins that have evolved against their caterpillars.

\section{Introduction}

The biodiversity on Earth is shaped by numerous factors including inter-organismal interactions that can result in coevolution of adaptive traits. For example, the coevolutionary interactions between plants and insects as described by Ehrlich and Raven (1964) has driven the diversification of plant defensive metabolites (Swain, 1977; Becerra, 2015). In turn, specialist herbivores have evolved detoxification mechanisms, which allow them to feed on their host plants despite these toxic metabolites (Berenbaum, 1983; Després et al., 2007), e.g. caterpillars of the monarch butterfly (Danaus plexippus) can feed on cardenolide-containing milkweeds (Cohen, 1985; Malcolm and Brower, 1989), and caterpillars of Pierinae and Plutella xylostella can feed on glucosinolate- 
containing Brassicaceae (Wittstock et al., 2004; Wheat et al., 2007; Heidel-Fischer and Vogel, 2015).

The role of plant defences against herbivore eggs has been understudied and underappreciated, especially in a coevolutionary context between herbivores and plants. The majority of studies on plant-insect interactions have focused on the feeding life stages of herbivorous insects. Yet, in almost half of the $\sim 400.000$ known herbivorous insects, especially lepidopteran and sawfly species, eggs may be the first life stage to come into contact with the targeted host plant. Indeed, plants can already perceive and respond physiologically to the presence of herbivore eggs before they hatch (Hilker and Fatouros, 2016). Plant defences against insect eggs may have evolved as an important first line of defence, as every insect egg being detected and killed, is one less herbivorous larva or adult insect feeding on the plant in the near future.

Different types of plant defences against insect eggs have been reported in more than thirty plant species including gymnosperms and angiosperms (both monocots and eudicots) (Fatouros et al., 2016). In response to insect egg deposition, plants can produce ovicidal substances (Seino et al., 1996), form neoplasms (Doss et al., 2000; PetzoldMaxwell et al., 2011) or express a hypersensitive response (HR)-like necrosis beneath the eggs (Shapiro and DeVay, 1987; Balbyshev and Lorenzen, 1997; Petzold-Maxwell et al., 2011; Fatouros et al., 2014). HR-like necrosis is an egg-killing defence leading to eggs desiccating and/or falling off the leaf. It has so far been observed in plants of the Pinaceae (Bittner et al., 2017), Poaceae (Yang et al., 2014), Fabaceae (Garza et al., 2001), Solanaceae (Balbyshev and Lorenzen, 1997; Petzold-Maxwell et al., 2011) and Brassicaceae (Shapiro and DeVay, 1987; Fatouros et al., 2014; Pashalidou et al., 2015a; Griese et al., 2017) families. To understand whether egg-killing traits have evolved as counteradaptations to specialist herbivores and their detoxification mechanisms, phylogenetic occurrence of the HR-like egg-killing trait across these plant families and reciprocal insect pest-clades need to be investigated.

Sequence-based phylogenetic analysis (Al-Shehbaz, 2012; Guo et al., 2017; Huang et al., 2015) has established that the Brassicaceae family is split into a core clade containing 3680 species, which is subdivided into three major lineages, and a smaller sister clade containing only the genus Aethionema (61 species) (Beilstein et al., 2006; Beilstein et al., 2008). The model plant Arabidopsis thaliana is a representative of Lineage I and the Brassica crop plants are representatives of Lineage II. Lineage III is a smaller 
group mostly restricted to Asia and lacking a model or crop species. Cleomaceae is the sister family of the Brassicaceae (Hall et al., 2002). Within the Brassicaceae, defences against feeding herbivores and the genetic basis of this defence have intensively been studied (Graser et al., 2000; Rask et al., 2000; Windsor et al., 2005; Xue et al., 1992). Aliphatic glucosinolates evolved as defensive compounds near or at the origin of the Brassicales clade and became more diverse and complex with plant species radiation. While these compounds play an important role in defending the plants against herbivory, many feeding insects have specialized and evolved effective glucosinolate detoxification and/or excretion mechanisms (Heidel-Fischer and Vogel, 2015; Erb and Robert, 2016; Heidel-Fischer et al., 2019; Winde and Wittstock, 2011).

The Pieridae butterflies (whites and sulphurs), including approximately 1000 species today (Wahlberg et al., 2014), use host plants belonging to two major plant orders, the Fabales (Fabaceae) and Brassicales (Brassicaceae, Resedaceae, Capparaceae and Cleomaceae), although some species in certain clades also shifted to Rosales (Rhamnaceae, Rosaceae) or Santalales (Edger et al., 2015). Recent phylogenetic reconstruction of the Pieridae indicates that the ancestral host appears to be a fabaceous with multiple independent shifts to other orders. While the Dismorphiinae and nearly all Coliadinae are Fabales feeders, the sister to the Coliadinae, Pierinae, primary feed on Brassicales (Wheat et al., 2007; Braby et al., 2006). The latter, thus, represent a single origin of feeding on glucosinolates producing plants.

Shortly after the initial evolution of the order Brassicales, some ancestral Pierinae evolved nitrile-specifier proteins (NSPs) that detoxify glucosinolates. This enabled a host shift from their prior Fabaceae hosts to the Brassicales c. 80 million years (Myr) ago (Edger et al., 2015). Likewise, the evolution of glucosinolate sulfatase in Plutella xylostella allowed the caterpillars of this moth to feed on Brassicaceae (Heidel-Fischer and Vogel, 2015; Wheat et al., 2007). It has been shown that speciation-rate shifts, as well as genome-duplication events with gene birth-death dynamics occurred in both Brassicales and Pierinae, usually following a key defence (glucosinolates) or counterdefence (NSPs) invention in one of the coevolutionary partners (Edger et al., 2015). Defence responses targeting eggs might have added a new layer of traits evolved in response to herbivore specialization. To pinpoint the evolution of transitions and innovations, it is necessary to investigate the trait(s) of interest in a proper phylogenetic context. 
Defence responses induced by cabbage white butterfly eggs have mainly been studied in the model plant Arabidopsis thaliana and the black mustard Brassica nigra (Little et al., 2007; Pashalidou et al., 2015b; Fatouros et al., 2014; Firtzlaff et al., 2016; Paniagua Voirol et al., 2020; Stahl et al., 2020). In A. thaliana, Pieris brassicae and P. rapae eggs activate a plant immune response, that resembles pattern-triggered immunity (PTI) against pathogenes. It includes expression of defence genes e.g. pathogenesis-related genes ( $P R I)$, and accumulation of reactive oxygen species (ROS), and a local cell death response. However, a visible necrosis is not expressed and egg-killing has never been shown (Reymond, 2013; Little et al., 2007). Egg killing due to a strong necrosis has been shown for the black mustard B. nigra in response to Pieris spp. Within B. nigra, there is variation in frequency and severity of HR-like necrosis between accessions (Fatouros et al., 2014; Pashalidou et al., 2015a; Griese et al., 2017).

The current study explores whether HR-like necrosis evolved as a specific response to pierid egg deposition in a subset of Brassicaceae. So far, no effort has ever been made to map the phylogenetic history of any egg defence trait for any plant family. Doing so would be a first necessary step to show an adaptive response to egg deposition. We investigated the phylogenetic occurrence of HR-like necrosis in the Brassicaceae (mainly lineage I and II) and three species in the Cleomaceae as well as explored the reciprocal phylogenetic co-occurrence in the Pieridae clade. We tested egg wash applied on $B$. nigra plants from four Pieris butterflies (Pierinae) and five relatives, i.e. Anthocharis cardamines (Pierinae) feeding on Cardamine plants of Lineage I, Aporia crataegi feeding on Prunus spp. (Rosaceae), Gonepteryx rhamni (Coliadinae) feeding on Rhamnus plants (Rhamnaceae), Colias spp. (Coliadinae) and Leptidea sinapis (Dismorphinae) both feeding on different species of the Fabaceae. As an outgroup, we used the butterfly Aglais io (Lepidoptera: Nymphalidae) that feeds on Urtica plants (Urticaceae). Additionally, we studied elicitation of egg wash of two moths, Mamestra brassicae (Noctuidae) and Plutella xylostella (Plutellidae), both feeding on Brassicaceae. Besides screening for HR-like necrosis, we investigated whether important components of plant defences, such as $P R 1$ gene expression, cell death and accumulation of ROS, correlated with the egg-induced necrosis. We tested the effect of HR-like necrosis on survival of singly-laid Pieris eggs in different plant species under both field and greenhouse conditions. Finally, we hypothesize the evolution of potential counteradaptations to egg-killing by some pierid butterflies. 
Specifically, we addressed the following questions: (i) Is HR-like necrosis induced in a clade-specific manner within the Brassicaceae? (ii) Are HR-like necrosis and other defence responses induced by eggs specific to a particular clade of butterfly species (e.g. genus, subfamily or family) and/or specific to species that coevolved with the Brassicaceae? And (iii) Is the observed necrosis lowering egg survival under glasshouse and field conditions?

\section{Results}

Origin of HR-like necrosis in the Aethionema and core Brassicaceae Out of 31 Brassicales plant species used this study, five species responded significantly with HRlike necrosis to $P$. brassicae egg wash. This included species of the tribe Brassiceae and of the genus Aethionema (Figure 5.1a). In the tribe Brassiceae, egg wash treatment significantly enhanced expression of HR-like necrosis in specific accessions of four species: B. napus (in 25-86\% of tested plants), B. nigra (63-83\%), B. olerace (20-40\%) and $C$. hispanica (0-86\%). HR-like necrosis of Aethionema arabicum varied among the tested accessions between 0 and 60\% (Table 5.A.5). There was no significant induction of HR-like necrosis after egg wash treatment for all other plant species tested compared to control leaves. HR-like necrosis was expressed at low frequency and low severity in several other species of Lineage II. For example, 30\% of wild Lunaria annua plants showed a weak HR-like necrosis, which was almost significant ( $\mathrm{P}=0.05$; Table 5.A.5). Necrosis was expressed rarely in species in lineage I and III (Fig. 5.1a): only in single plants of some accessions and once in Aethionema carneum. 

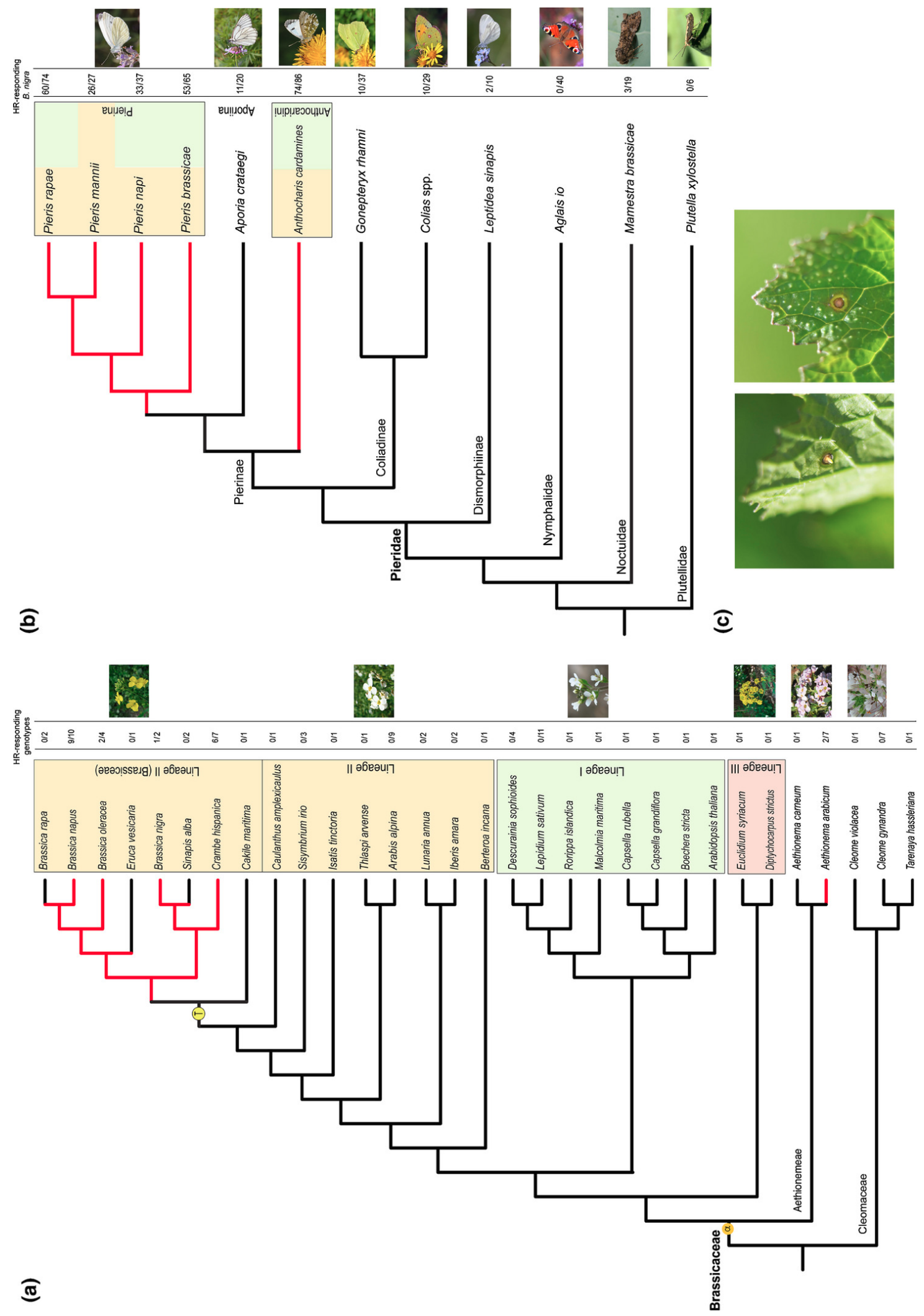

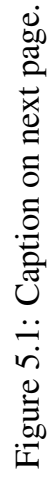


Chapter 5 Coadaptation of HR-like necrosis

Figure 5.1: (Previous page.) Presence of HR-like necrosis mapped on phylogenetic trees of Brassicaceae (a) and Pieridae (b). Red lines: HR-like necrosis present; black lines: HR-like necrosis absent in the majority of tested genotypes; a) Screening of HR-like necrosis by $P$. brassicae egg wash in 28 Brassicaceae species and three Cleomaceae species based on the published phylogenies (Guo et al., 2017; Huang et al., 2015). Different lineages are coloured in orange (lineage II/tribe Brassiceae), brown (lineage II), green (lineage I) and red (lineage III). The whole genome duplication WGD $(\alpha)$ and genome triplication (T) the Brassiceae tribe specific events are marked on the tree. (b) Elicitation of HR-like necrosis by pierid egg wash or eggs in $B$. nigra leaves by different butterfly and moth species shown on phylogenies based on (Kawahara et al., 2019; Wiemers et al., 2020). Responses of the pierid species were compared to the nymphalid Aglais io, the noctuid moth Mamestra brassicae and the plutellid moth Plutella xylostella. Coloured boxes represent species of the Brassicaceae used as main host plants by the butterflies in the same coloured box. Brassicaceae and Lepidoptera (sub)families are written on their nodes where they separate from the rest of the clades. Photos of butterflies and moths taken by Jitte Groothuis, Hans M. Smid, Tibor Bukovinszky, Zeynel Cebeci, Charles J. Sharp, Juergen Mangelsdorf. (c) HR-like necrosis induced by a single Pieris sp. egg in B. nigra taken from the under- and upper-side of the leave (credits N. E. Fatouros).

Elicitation of HR-like necrosis by pierid species adapted to Brassicaceae The elicitation of HR-like necrosis in B. nigra by egg deposition or egg wash of nine lepidopteran species was tested: eight pierid and one nymphalid species (Figure 5.1). First, we assessed the HR frequency and severity (scored from 0 , no symptoms to 3 , strong necrosis) in B. nigra in response to egg deposition or egg wash of four Pieris species, four relatives (A. cardamines, Colias spp., G. rhamni, L. sinapis) and A. io as outgroup. Eggs or egg wash of $P$. brassicae, $P$. napi, $P$. rapae, $P$. mannii and $A$. cardamines induced a high fraction of HR-like necrosis in B. nigra $(0.82 \pm 0.06 ; 0.75 \pm 0.06 ; 0.86 \pm 0.14 ; 0.89 \pm$ 0.05 respectively) and all induced with high severity (Table 5.A.6). When several populations were available for butterfly species, all populations elicited HR-like necrosis in similar frequency (GLM: $\chi^{2}=1.36, \mathrm{df}=3, P=0.71$ ) and severity (GLM: $\chi^{2}=2.60$, $\mathrm{df}=3, P=0.46)$. The fraction and severity of HR-like elicited by G. rhamni, Colias spp. and L. sinapis was generally lower than HR-like induced by the eggs of Pieris spp. and A. cardamines (Table 5.A.6 and 5.A.7). Moreover, the responses induced by the egg wash of these non-brassicaceous Pieridae in plants appeared to be chlorosis instead of necrosis (Figure 5.A.1). The egg wash of $A$. io, induced no symptoms on B. nigra (Table 5.A.6). 
HR-like necrosis severity correlates to $P R 1$ defence gene expression We then performed an experiment to compare the response induced by egg wash of Pierinae and their relatives including two moths that can feed on Brassicaceae: P. xylostella and M. brassicae. As expected, we observed significant differences in HR-like frequency between the Pierinae and other butterfly and moth species (GLM: $\chi^{2}=28.3$, df $=9, P<$ 0.001; Table 5.A.7) and in HR severity (Kruskal-Wallis $\mathrm{H}=133.37$, $\mathrm{df}=9, P<0.001$; Figure 5.2a). In this experiment, $P$. rapae and $P$. mannii induced HR-like response in all plants tested with high severity (2.88 \pm 0.01 and $2.95 \pm 0.01$, Figure 5.2a, Table 5.A.7). Anthocharis cardamines also induced high HR severity on $65 \%$ of the tested plants (1.55 $\pm 0.04)$. The more distantly related Pieridae G. rhamni and Colias spp. induced necrosis in only a few plants with low severity $(0.31 \pm 0.03$ and $0.60 \pm 0.04$, table 5.A.7), similar to that of the two moth species, and control treatment (Figure 5.2a). HR-like frequency and severity induced by A. crataegi was in between of that induced by Pieris spp. and Anthocharis and the more distantly related species (Figure 5.2a, Table 5.A.7).

Besides a HR-like necrosis, Pieris spp. eggs are also known to induce other defence response in $A$. thaliana and B. nigra, including $P R 1$ gene expression following egg deposition or egg wash treatment (Little et al., 2007; Fatouros et al., 2015). Consequently, we tested if egg washes of other butterfly and moth species also induced a defence response in $B$. nigra by measuring $P R 1$ gene expression. Interestingly, $P R I$ expression was significantly induced by egg wash of all butterfly and moth species tested, except $M$. brassicae. Nevertheless, there were significant differences in $P R 1$ induction between the different species (Kruskal-Wallis $\mathrm{H}=35.394$, $\mathrm{df}=8, P<0.0001$; Figure 5.2b). Expression of $P R 1$ correlated with HR severity and was significantly higher induced by $P$. rapae and $P$. mannii, and also, although less strongly, by A. cardamines (Figure 5.2b. Lower HR severity correlated with lower $P R 1$ expression. Notably, the Pierinae $A$. crataegi, which scored in between A. cardamines and G. rhamni for HR severity, also had an in-between $P R 1$ expression. Colias spp. induced $P R l$ the highest among the non-Pierinae species, but still about 100- fold lower expressed compared to $P$. mannii (Table 5.A.8). Interestingly, $P R l$ expression was also induced by $P$. xylostella egg wash, which showed no visual symptoms in these plants (Figure 5.2a).

HR-like severity correlates with increased $\mathrm{H}_{2} \mathrm{O}_{2}$ and cell death in a subset of Brassicaceae The screening for HR-like necrosis across different Brassicaceae revealed inter- 

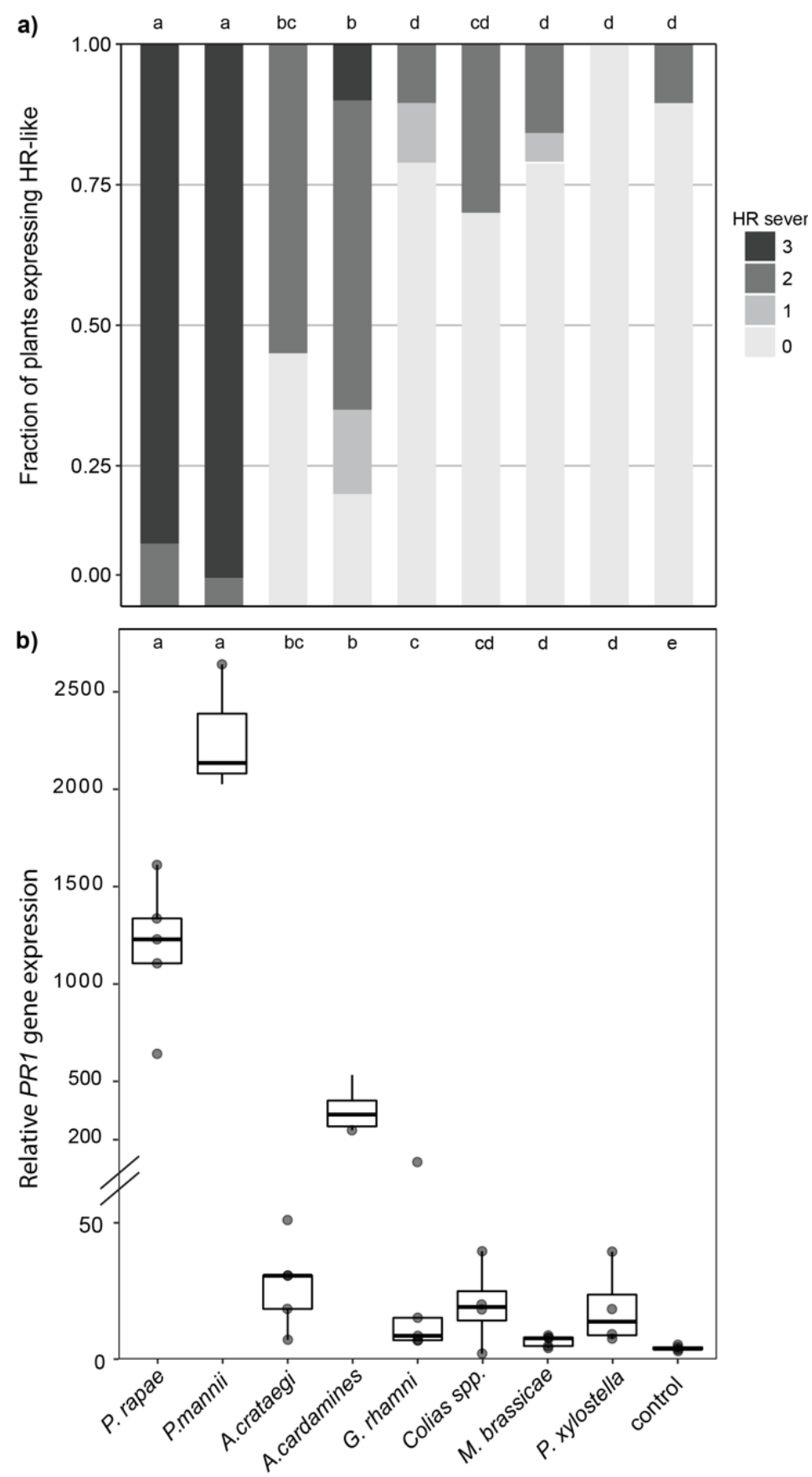

Figure 5.2: HR-like necrosis and $P R I$ gene expression induced by egg wash of different butterfly and moth species in B. nigra plants. For the experiment, two droplets of $10 \mu \mathrm{l} \mathrm{egg} \mathrm{wash} \mathrm{were}$ applied onto one of each plant. a) Fraction of plants expressing HR-like necrosis at different severities $(0=$ no response, $3=$ strong necrosis $)$. A total of $\mathrm{N}=18-20$ plants was tested per butterfly species, for P. xylostella, egg wash for only six plants was available to test for necrosis. b) Relative expression of $P R 1$ gene upon treatment with egg wash of different butterflies/moths or MES buffer as control. Transcript levels were measured by qRT-PCR on 4-5 biological replicates, each composed of 4 pooled individual plants. Letters denote differences in HR severity or mean transcript levels between different treatments (Wilcoxon rank sum test, $P<0.05$ ). 
specific variation in HR frequency (Table 5.A.5). In addition, we also observed variation in HR severity between plant species that showed high HR frequency. To quantify the differences observed, we then measured the area of necrotic tissue induced by $P$. brassicae eggs in three species: B. nigra, B. olerace and $C$. hispanica. We found that the necrotic area was largest in B. nigra, and significantly smaller in the other two species (ANOVA, $\mathrm{F}=17.028$, df $=2, P<0.001$, Table 5.A.9). Previously, Pieris brassicae eggs on A. thaliana were shown to induce components of plant immunity such as $\mathrm{H}_{2} \mathrm{O}_{2}$ and cell death despite absence of a visible HR-like necrosis (Little et al., 2007; GouhierDarimont et al., 2013). Therefore, we investigated to which extent the visible bigger or larger HR-like necrosis correlates with induction of $\mathrm{H}_{2} \mathrm{O}_{2}$ and cell death. Plants that showed a small HR-like necrosis, i.e. B. olerace and $C$. hispanica, exhibited a high accumulation of $\mathrm{H}_{2} \mathrm{O}_{2}$ and trypan-blue stained cell death compared to the extension of visible necrosis (Figure 5.A.2). Brassica nigra showed a strong visible necrosis, larger than $1 \mathrm{~mm}^{2}$ per 10 eggs, which extended further than the $\mathrm{H}_{2} \mathrm{O}_{2}$ and cell death accumulation (Figure 5.A.2). These results suggest that components of the plant immunity are induced regardless of the variation in HR-severity, and that $B$. nigra induces the visible HR-like response most strongly.

Effect of HR-like necrosis on Pieris spp. egg survival on different Brassicaceae species First, we monitored egg survival on wild B. nigra plants of Pieris spp. (P. napi and $P$. rapae) most abundant under natural field conditions in the Netherlands. Egg survival was $40 \%$ lower when eggs induced HR-like necrosis compared to survival of eggs that did not induce a leaf necrosis (GLM: $\chi^{2}=11.02$, df $=1, P<0.001$, Figure 5.3a), confirming previously reported results (Fatouros et al., 2014). Considering the variation in HR severity and HR frequency between plant species, we investigated the effect of HR severity in different species on Pieris egg survival. We tested egg survival on five plant species from the first screening under greenhouse conditions (Figure 5.1): three species that showed high HR frequency and contrasting HR severity (B. napus, B. nigra, and $C$. hispanica) and two species with low HR frequency (B. rapa and A. thaliana). HR-like necrosis significantly lowered the survival of singly laid $P$. brassicae eggs on all three plant species that previously showed high HR frequency (GLM: $\chi^{2}=38.41$, $\mathrm{df}=1, P<0.001$, Figure 5.3b). On $C$. hispanica plants egg survival was significantly lower than on B. napus plants (pairwise MWU:P $=0.006$, Figure 5.3b). Conversely, 
egg survival was not affected by HR-like necrosis for $B$. rapa that generally showed low HR severity (GLM: $\chi^{2}=2.61$, df $=1, P=0.14$, Figure 5.3c). On A. thaliana different accessions, were no visible HR-like necrosis was observed, egg survival was $100 \%$, the highest of all plant species tested (Figure 5.3d).

a)

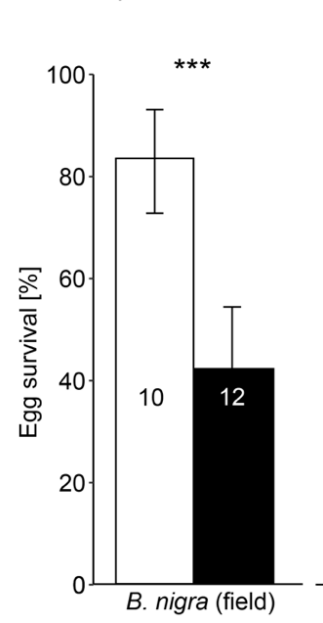

b)

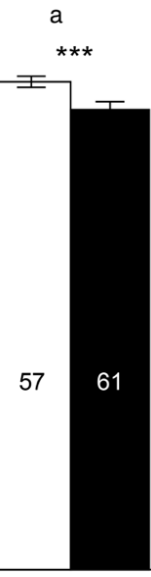

B.napus

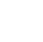

$a b$ b

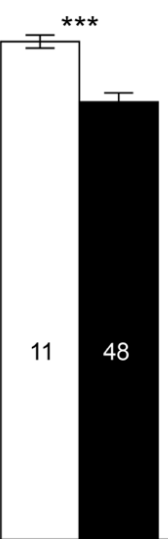

C.hispanica

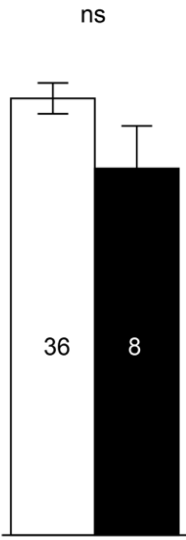

B.rapa d)

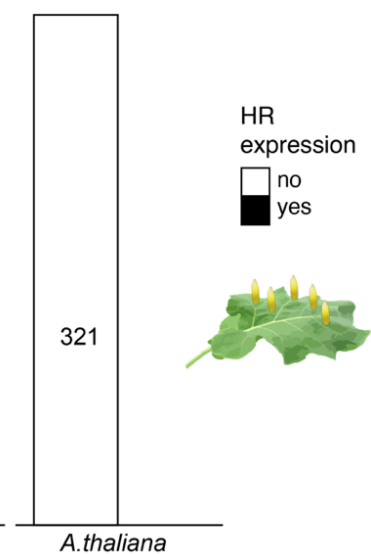

Plant species

Figure 5.3: Effect of HR-like necrosis on survival rates of singly laid Pieris eggs on different plant species. a) Effect of HR-like necrosis on egg survival in field conditions. Survey of $P$. napi and $P$. rapae eggs on $B$. nigra plants located near the river Rhine in Wageningen (NL). One to 13 eggs were sampled per plant. b)-d) Effect of HR-like necrosis on egg survival under greenhouse conditions. Three experiments were performed with singly laid $P$. brassicae eggs on different accessions of B. napus, B. nigra, $C$. hispanica (b) and B. rapa (c) as well as $P$. rapae eggs laid on $A$. thaliana (d). 10 single eggs were laid on each plant for experiment b), 5 single $P$. brassicae eggs on each plant for experiment c), a single $P$. rapae egg per plant for d). If a plant expressed HR-like necrosis under at least one egg it was counted as HR-expressing 'yes'. Different letters indicate significant differences (GLM,P<0.001). Egg survival represents mean $\pm \mathrm{SE}$ of hatched eggs for each plant. If a plant expressed HR-like necrosis under at least one egg it was counted as HR-expressing 'yes'. Asterisks indicate significant differences in egg survival between plants with or without HR-like necrosis. Different letters indicate significant differences in egg survival between plant species, without taking HR-like necrosis into account (GLM, ns: not significant, $* * P$ $<0.01, * * * P<0.001)$.

\section{Discussion}

Pierid butterflies and their brassicaceous host plants are a fascinating model system of coevolutionary interactions, and research so far has explored the evolutionary and genetic basis of these interactions by focusing on the diversifying selection on plant 
chemical defences, i.e. glucosinolates, and insect nitrile-specifier protein (NSP) detoxification genes (Edger et al., 2015; Nallu et al., 2018). Here, we attempt for the first time to map the phylogenetic history of egg induction (i.e. hypersensitive response (HR)like necrosis) as a plant defence trait and its reciprocal co-occurrence in the herbivore clade. We show that a strong HR-like necrosis induced by Pieris eggs most frequently occurs in one clade within the Brassicaceae. Half of the tested plant species from the Brassiceae tribe in Lineage II express HR-like necrosis with high frequency in response to $P$. brassicae egg wash. The visual necrosis was accompanied with increased levels of $\mathrm{H}_{2} \mathrm{O}_{2}$ in three representative $\mathrm{HR}+$ plant species. Of the tested Brassica and Crambe spp. (tribe Brassiceae), HR-like necrosis lowered egg survival both under natural and glasshouse conditions, except for $B$. rapa that does not express a strong HR-like necrosis as e.g. C. hispanica or B. nigra. Interestingly, egg survival was generally lower on $B$. rapa, which could hint to plant defences other than HR-like necrosis or non-ideal circumstances for Pieris eggs. Furthermore, we showed for the first time that only egg wash from species of the subfamily Pierinae, that specialized on the Brassicaceae, i.e. Pieris butterflies and A. cardamines, elicit a strong HR-like necrosis and high levels of PRI defence gene on B. nigra. Species that are specialized on Fabaceae or Rhamnaceae from the Coliadinae, Colias spp. and G. rhamni, and Dismorphiinae, L. sinapis, elicited a weak necrosis or sometimes just a chlorotic response similar to that of Solanum dulcamara to Spodoptera eggs (Geuss et al., 2017). Our results suggest that the elicitation of strong HR-like necrosis by Pieris eggs may have a single origin in the ancestor of the Brassicaceae (Fig. 5.1) with variation in the frequency and severity of the trait between species and accessions, whereas the trait is most strongly expressed in the Brassiceae tribe. Moreover, we show that B. nigra plants specifically evolved strong HR-like necrosis to the eggs of those pierid species that evolved effective glucosinolate detoxification mechanisms. 
Evolution of HR-like necrosis in Brassicaea and Aethionema Four out of eight tested Brassicaea species, as well as one Aethionema species showed consistent HR-like necrosis to Pieris egg wash in at least one of the genotypes tested. In other plant species in the Brassicaceae, we found no consistent induction of HR-like necrosis by Pieris egg wash. It is unlikely that the genome triplication event specific to the Brassiceae clade is the only factor involved in the evolution of HR-like necrosis, as one Aethionema species responded to Pieris eggs with a strong necrosis. There may be ecological reasons, such as overlap in spatial distribution between butterflies and plant species, that can explain why HR-like necrosis appears more severe and at higher frequency within the Brassiceae and Aethionema. In fact, besides many of the tested Brassiceae plants, Aethionema are natural host plants for Pierinae species as well. Pieris ergane, Anthocharis gruneri and Euchloe ausonia specialized on Aethionema species in their south eastern European habitat (Tolman and Lewington, 2009). Because of high abundances of Pierinae species occurring on Aethionemeae, it could be that species of this basal clade of the Brassicaceae retained a severe HR-like necrosis as an effective trait against eggs of these butterfly species.

In other plant species tested, occasionally a single plant showed a light HR-like necrosis. These plants might be able to detect insect eggs and respond with a general immune response, as recently shown for A. thaliana (Gouhier-Darimont et al., 2013; Gouhier-Darimont et al., 2019; Stahl et al., 2020). Alternatively, it could be a falsepositive response due to a contamination or a general stress response, as in rare cases, also control wash induced a weak necrosis. In general, not all tested plant species within the Brassiceae tribe within Lineage II expressed HR-like necrosis though. Variation for the HR-like necrosis trait between genotypes of one species has been observed before (Griese et al., 2017; Pashalidou et al., 2015a). It is therefore possible that we may have missed HR-like necrosis expressing plants because of the selection of non-responsive or less sensitive genotypes for some of the plant species or genus. For example, Sinapis alba did not show HR-like necrosis (Figure 5.1) but previous work on the close relative Sinapis arvensis showed that eggs of $P$. rapae and $P$. brassicae strongly induced HR-like necrosis (Griese et al., 2020).

For the model species A. thaliana, a few accessions other than the ones included in this study did show a chlorosis and/or some necrosis to P. brassicae eggs (Reymond, 2013; Groux et al., 2020). For half of the tested species here, only one genotype was 
tested, increasing the likelihood of selecting only nonresponsive ones (Fig. 5.1a; Table 5.A.1). Some plant species and accessions might have lost the ability to express HR-like necrosis, or only do so rarely. Those plants may be less frequently used as host plants for pierid butterflies, for example because of a phenological mismatch between the plant species and its potential specialist herbivores. This mismatch can be especially true for species belonging to Lineage I and III. For example, in central Europe A. thaliana is usually not attacked by pierid butterflies, as it is rather small and usually completes its life-cycle before pierid caterpillars could develop on the plant (Harvey et al., 2007). Notably, A. cardamines was observed to deposit eggs on A. thaliana in North Sweden where both life cycles briefly overlap (Wiklund and Friberg, 2009). Yet, P. rapae eggs did not induce a leaf necrosis lowering Pieris egg survival on Swedish accessions of A. thaliana (Figure 5.3d), neither did we observe a visible necrosis on the commonly used genotype Col-0 in our experiments when using P. brassicae egg wash (Figure 5.1, table 5.A.5). The observed variation in HR-like necrosis between genotypes of the same species suggests that expression of this trait might have negative effects on plant fitness and only evolves with high herbivore pressure. Alternatively, variability in a defence trait might in itself be defensive as postulated by the moving-target strategy to counteract the development of efficient plant defensive responses by herbivores (Adler and Karban, 1994). Phenotypic variation in HR-like necrosis to eggs was previously suggested to be part of such a moving-target game (Hilker and Fatouros, 2015).

Counteradaptations of brassicaceous-feeding Pierinae species Previous work has shown that the NSP glucosinolate detoxification gene was a key innovation in the ancestral Pierinae enabling them to shift host plant from Fabaceae to Brassicaceae (Edger et al., 2015). Interestingly, induction of a strong, egg-killing HR-like necrosis and high levels of PRl gene expression seem to be specific to Pieris (Pierini) and Anthocaris (Anthocharidini) species belonging to the Pierinae subfamily that colonized the Brassicales some 50 Myr ago (Wheat et al., 2007). We suggest that this may be a counteradaptation of some brassicaceous plants to the nitrile-specifier genes that evolved in the Pierinae. Because those nitrile-specifier genes detoxify glucosinolates and enabled butterflies of those lineages to conquer the Brassicaceae, a new and separate plant defence mechanism might have evolved. Reciprocally, pierid butterflies also may have found ways to counteradapt to the egg-killing HR-like necrosis. 
Molecular and cellular responses to insect eggs When the response to Pieris eggs was first described in B. nigra some 30 years ago (Shapiro and DeVay, 1987), the induction of cell death was only known from biotrophic pathogens, whose spread is limited by the death of cells. It is now clear that cell death is a common phenomenon with many different causes, that can be induced by several different biotic interactors, including insects and nematodes (Balint-Kurti, 2019). In our study, we found that HR-like cell death is induced in the Brassiceae tribe by P. brassicae egg wash, and in B. nigra by all Pierinae species tested. To understand if the mechanism of this response is shared between these different plant species, and in response to the different butterfly species, detailed knowledge on the molecular responses to eggs, genes that are involved in the detection and recognition, and elicitors of the response are required. An in-depth molecular characterization of the Pierinae egg-induced HR-like necrosis compared to other microbial-induced HR goes beyond the aim of this study. Nevertheless, we have attempted to start with a description of the molecular response to insect eggs by studying trypan blue-stained cell death and accumulation of reactive oxygen species (ROS) in three plant species, and $P R I$ expression in B. nigra towards nine insect species. In this study, ROS accumulation and cell death were induced in all plant species tested, whereas the strong HR-like necrosis and high PRI expression was specific to B. nigra and to Pierinae insect species. It is possible that also in other species and accessions in the Brassicaceae that we have not investigated closely, a general immune response lacking a strong cell death is activated by Pieris eggs, as was shown for A. thaliana (Col-0) (Gouhier-Darimont et al., 2013; Gouhier-Darimont et al., 2019; Stahl et al., 2020; Valsamakis et al., 2020). Our data suggest that the strong HR-like necrosis is always accompanied by ROS and high PRl expression. However, because our histochemical stainings involved only three plant species (B. nigra, B. oleracea, C. hispanica), our observations may have been confounded with possible plant interspecific variation in the $\mathrm{H}_{2} \mathrm{O}_{2}$ and cell death-inducing pathways. To understand whether the different species in which $P$. brassicae egg wash induce cell death share the same or similar mechanisms, requires the identification of genes involved in egg detection and downstream defence response activation in the responsive plant species identified in this study. At the moment, we are undertaking genetic studies to identify putative plant receptors required for perception of Pieris eggs in different Brassica spp. 
Elicitor of HR-like specific to Pierinae eggs Although induction of strong HR-like necrosis and high levels of $P R 1$ gene expression in B. nigra was specific to Pieris and Anthocharis species, neither the non-Pierinae butterflies nor the moth species tested induced a strong HR-like necrosis on B. nigra (Figs 5.1, 5.2; Table 5.A.6). This suggests that the elicitor for HR-like necrosis is one or several molecules found only in Pierinae eggs, rather than a general molecule present in (all) butterfly eggs. The differences in the severity of HR-like necrosis elicitation between different Pierinae species could either be caused by quantitative differences of these elicitor(s), or by changes in their chemical composition. In A. thaliana, eggs from distantly related insect species were recently shown to release phosphatidylcholines (PCs) that induce a general immune response (i.e. pattern-triggered immunity) involving salicylic acid and $\mathrm{H}_{2} \mathrm{O}_{2}$ accumulation (Little et al., 2007; Gouhier-Darimont et al., 2013; Gouhier-Darimont et al., 2019; Stahl et al., 2020). A lectin receptor kinase, LecRK-I.8, might be involved in early perception of eggs from two widely divergent species, P. brassicae and Spodoptera littoralis (GouhierDarimont et al., 2019). Interestingly, low PRI expression was induced by egg wash of Coliadinae butterflies and P. xylostella in B. nigra also in our experiments. These results support a model where a general egg molecule (PCs) is detected by many plants (including A. thaliana and B. nigra) and a Pierinae-specific egg-associated molecular pattern (EAMP) may be detected specifically by the Brassiceae tribe. This would be similar to the detection of microbe-associated molecular patterns (MAMPs) by the plant immune system (van der Burgh and Joosten, 2019). An exciting next step would be the identification of the Pierinae-specific elicitor(s). Currently, we are analysing the chemical composition of egg wash from different butterfly species to identify the compounds inducing HR-like necrosis.

In conclusion, our findings demonstrate that various Brassicaceae plants can mount defences against insect eggs and that these might be under similar selective pressures as plant defences against feeding insects. A coevolutionary arms race between Pieris butterfly eggs and plant species within the Brassiceae clade as well as species within the sister clade Aethionema is likely to have occurred. These plants make use of necrotic lesions to lower egg survival and in this way might have evolved a new mechanism, possibly co-opted from pre-existing plant immunity mechanisms, to combat eggs of specialist herbivores adapted to their host plants' toxins. 


\section{Material and Methods}

Plants and insects For our study, we obtained seeds of thirty-one species in the Brassicales (28 Brassicaceae and 3 Cleomaceae), from various sources. For each plant species, between one and eleven accessions were grown (Table 5.A.1). Per accession, between three and seventeen plants were treated with egg wash to assess elicitation of a HRlike response by Pieris brassicae. Brassica nigra plants were used to assess elicitation of the HR-like necrosis by different butterfly species. Finally, egg-killing was tested for six plant species. In preliminary trials, plant species with unknown developmental times were grown to assess their germination and flowering after sowing. Then, plants were sown in a scheme to ensure that they had reached similar life stages (i.e. vegetative growth) when used for experiments. Therefore, plants were between three and six weeks old when being treated with butterfly eggs or egg wash. I order to assess the occurrence of HR-like necrosis across the selected Brassicales species, we used a wash of Pieris brassicae eggs. To assess induction of HR-like necrosis on B. nigra plants, we used egg deposition by different butterfly/moth species and populations (for details, see Methods 5.A1 and Table 5.A.2).

Egg wash preparation Not all butterflies and moths used in this study naturally deposit eggs on all plant species that were selected. In order to be able to test those species and screen a large number of plants efficiently, we developed a method to prepare an egg wash that can be used to mimic oviposition as plant treatment. The development and testing of this method will be submitted elsewhere. We showed that there is no difference in the symptoms induced on B. nigra leaves between eggs and egg wash of P. brassicae (Caarls et al., 2021). For this method, female butterflies of P. brassicae, $P$. rapae or $P$. napi, and $M$. brassicae moths were persuaded to lay eggs on paper that was pinned to the underside of a leaf. Wash from Aglais io, A. cardamines, A. crataegi, Colias spp., G. rhamni, and L. sinapis eggs was made by carefully removing eggs from leaves or inflorescences. Collected eggs were counted and washed overnight in MES buffer. Concentrations of the egg washes we re adjusted based on the size of the eggs used (for details see Methods 5.A2). 
Phenotyping of HR-like necrosis on Brassicales species Experiments were carried out in a greenhouse compartment $\left(22-27^{\circ} \mathrm{C}, \mathrm{RH}: 50-90 \%, 16 \mathrm{~h}: 8 \mathrm{~h}\right.$, light : dark). For the screening of thirty-one Brassicales plant species, $5 \mu \mathrm{l}$ of $P$. brassicae egg wash was pipetted on a fully mature leaf (the third or fourth leaf from the top) of each plant. Another fully matured leaf (the third or fourth from the top) received pure water containing Tween 20 as a control. After four days, leaf disks were harvested of the area where egg wash had been applied using a $1 \mathrm{~cm}$ cork borer and put in a rectangular Petri dish with wet blue filter paper. Pictures were taken using a Dino-Lite digital microscope (AnMo Electronics Corporation). These pictures were visually scored for expression of HR-like necrosis (see below).

Elicitation of HR-like necrosis by diverse Pieridae species Female butterflies of $P$. brassicae (two populations), P. napi and P. rapae (two populations) were allowed to lay between five to ten eggs on two different B. nigra accessions (SF19 and SF48) (Table 5.A.1) (Griese et al., 2017). A. io, A. cardamines, Colias sp. and G. rhamni egg wash was tested on both B. nigra accessions. A. crataegi, L. sinapis, P. mannii, M. brassicae and P. xylostella wash was tested on B. nigra accession SF48. After $4 \mathrm{~d}$, HR-like necrosis was scored using a scoring system previously described by Griese et al. (2017).

\section{Pathogenesis-related protein 1 (PR1) gene expression by diverse butterfly and moth}

species In order to measure $P R 1$ gene expression, $10 \mu \mathrm{l}$ egg wash of $P$. rapae, $P$. mannii, A. crataegi, A. cardamines, G. rhamni, Colias spp., M. brassicae and P. xylostella was pipetted on the abaxial side of $20 \mathrm{~B}$. nigra (SF48) leaves per butterfly/moth species except for $P$. xylostella where egg wash for six plants was available. After $24 \mathrm{~h}$, two $6 \mathrm{~mm}$ diameter leaf disks were taken from egg wash application site and snap frozen in liquid nitrogen. $P R I$ transcript levels were measured on 5 biological replicates composed of 4 pooled individual plants. RNA isolation according to (Oñate-Sánchez and VicenteCarbajosa, 2008), real-time quantitative PCR analysis and primers are described in detail in Methods 5.A3 and Table 5.A.3.

Histochemical staining Pieris brassicae females were allowed to lay two egg clutches of 5-20 eggs on a single leaf per plant. From every plant, one clutch was used for histochemical staining $\left(\mathrm{H}_{2} \mathrm{O}_{2}\right.$ or cell death) whereas the other one was used to score the 
necrotic leaf area. Samples were taken at 48,72 or $96 \mathrm{~h}$ after oviposition by taking a $10 \mathrm{~mm}$ diameter leaf disc around the egg clutch.(for details, see Methods 5.A4).

Pieris spp. egg survival on HR-like expressing plants under greenhouse condition Experiments were done under long-day greenhouse conditions $\left(21 \pm 5^{\circ} \mathrm{C}, \mathrm{RH}\right.$ : 45-70\%, $16 \mathrm{~h}: 8 \mathrm{~h}$, light : dark) for B. nigra, B. napus, B. olerace, B. rapa and $C$. hispanica. Pieris brassicae females were manipulated to lay five to fifteen separated eggs (i.e. not touching each other) on all lines previously used in the screening of Brassicaceae species. The number of hatching and non-hatching eggs were counted to measure egg survival rates. Previously, P. brassicae egg survival was only affected when eggs were laid singly, not touching each other (Griese et al., 2017). The eggs were left on the plant and four days after oviposition HR-like necrosis was scored as present or absent. Egg survival on Arabidopsis thaliana, plants were reared under short-day greenhouse conditions $\left(21 \pm 4{ }^{\circ} \mathrm{C}, \mathrm{RH}: 70 \%, 16 \mathrm{~h}: 8 \mathrm{~h}\right.$, light : dark) to control for fast flowering. Seeds from 36 different Swedish accessions of A. thaliana were obtained from the HapMap population (http://bergelson.uchicago.edu/wp-content/uploads/2015/ 04/Justins-360-lines.xls). Pieris rapae females were allowed to lay a single egg on one leaf per plant according their natural behaviour. After five days, survival of eggs was noted by counting the number of hatched caterpillars.

Pieris spp. egg survival assessed by field survey It has been shown that HR-like necrosis has weaker effects on egg survival under glasshouse than under natural conditions (Fatouros et al., 2014). A survey was conducted to record survival of $P$. rapae and $P$. napi eggs on B. nigra plants from a natural population (for details, see Fatouros et al. (2014), and Methods 5.A5).

Phylogenetic trees of Brassicales and Pieridae species We used a consensus tree based by two recent studies (Huang et al., 2015; Guo et al., 2017) to place our tested Brassicales species accordingly. Both studies analyzed representatives of the three distinct linages of the core Brassicaceae clade and the first-branching Aethionema and the outgroup Cleomaceae (For details see Methods 5.A6 and Table 5.A.4).

We mapped the HR-like necrosis induced by the tested butterfly species according to two recent studies: a phylogenomic analysis of Lepidoptera (Kawahara et al., 2019) 
and phylogenetic analysis of the Papiolionoidea (Wiemers et al., 2020). The first study contained 994 taxa, whereas the second analyzed 496 extant butterfly species in Europe using mitochondrial gene $C O I$ and $\leq 11$ nuclear gene fragments. The European butterflies used were split in 12 subclades. The Pieridae were considered as a single clade and the Nymphalidae divided into seven subclades (Wiemers et al., 2020).

Statistical analysis Statistical analyses were done using R (R Core Team, 2016). For the screening of plant accessions, contingency tables and $\chi^{2}$-tests were used to determine which plant species/genotypes significantly expressed HR-like necrosis after egg wash treatment compared to the control treatment. The contingency tables for the $\chi^{2}$ tests were constructed with: the number of egg wash-treated leaves expressing HR-like necrosis; the number of egg wash-treated leaves not expressing HR-like necrosis; the number of control wash-treated leaves expressing HR-like necrosis; and the number of control wash-treated leaves not expressing HR-like necrosis. With this set-up, all plant accessions within each plant species were tested independently.

Egg survival was analysed using binomial generalized linear models (GLMs) in which first all variables (plant species, flowering state, HR expression and all interactions between the factors) were used. Based on Akaike information criterions (AICs), unnecessary variables were removed to obtain a more parsimonious model (plant species, HR expression and interaction). Subsequently, EMMEANS test (R package EMMEANS) or Mann-Whitney-U tests were performed as post-hoc tests.

Differences in induction of HR-like necrosis by different butterflies were tested using binomial GLMs and, to test differences in strength, GLMs with Poisson distribution. Dunn tests with Bonferroni-Holm correction were used as post-hoc tests. For difference in HR-severity Kruskal-Wallis followed by post-hoc Wilcoxon Rank Sum test with Benjamini \& Hochberg correction were performed.

Quantification of HR-like necrosis and histochemical staining for each plant species were compared with a Student's t-test. Difference in HR-like necrotic area between plant species was analysed with ANOVA, followed by Tukey post-hoc test with BenjaminiHochberg correction. Gene expression of PRl was analysed using Kruskal-Wallis followed by post-hoc Wilcoxon Rank Sum test with Benjamini-Hochberg correction. For all the statistical analysis involving comparison of mean values (egg survival, histochemical staining, gene expression), the choice of parametric or non-parametric methods was 
motivated after checking the assumptions of normality (Shapiro-Wilk normality test) and homogeneity of variances (Fligner-Killeen test) on the raw data.

\section{Acknowledgements}

We thank the employees of Unifarm (Wageningen University and Research) for rearing and caring of the plants used in the experiment. We are thankful to Pieter Rouweler, André Gidding, and Frans van Aggelen for rearing the Dutch Pieris butterflies used in the experiment. Guusje Bonnema (WUR, Plant Breeding), the Leibniz-Institut für Pflanzengenetik und Kulturpflanzenforschung (Germany) and the BMAP consortium are thanked for the seeds. Furthermore, we thank Prof. Miltos Tsiantis from the Department of Comparative Development and Genetics, Max Planck Institute for Plant Breeding Research for kindly providing $C$. hirsuta seeds, used as host plants for $A$. cardamines. The authors thank Marcel Dicke and Monika Hilker for reading and commenting on an earlier version of the manuscript.

Funding This research has been made possible by funding of the Dutch Research Council (NWO) to N.E.F. (NWO/TTW VIDI 14854 and connected Aspasia). 


\section{A Supplementary material}

Methods S1: Butterflies and moths Two populations of $P$. brassicae L., P. napi L. and $P$. rapae L. were tested (Table 5.A.2).Furthermore, we tested egg wash from one population of P. mannii Mayer, three populations of Anthochariscardamines L., one population of Aporia crataegi L., one population of Leptidea sinapis L., one population of Gonepteryx rhamni L. and Aglais io L. (Lepidoptera: Nymphalidae), and two moths species Plutella xylostella L. (Lepidoptera: Plutellidae) and Mamestra brassicae L. (Lepidoptera: Noctuidae) (Table 5.A.2). Finally, survival was measured for eggs of P. brassicae, P. napi and P. rapae. Pieris brassicae, M. brassicae and P. xylostella were reared on Brussels sprouts (B. oleracea var. gemmifera cv. Cyrus) in a climatized room $\left(21 \pm 1{ }^{\circ} \mathrm{C}, 50-70 \% \mathrm{RH}, \mathrm{LD} 16: 8\right)$. Pieris mannii, $P$. napi, and P. rapae were collected outside and reared in a greenhouse $\left(21 \pm 4{ }^{\circ} \mathrm{C}, 60-80 \% \mathrm{RH}, \mathrm{LD} 16: 8\right)$, either on flowering Iberis spp. plants (P. mannii) or Brussels sprouts. One population of A. cardamines was obtained from thebutterfly farm Farma Motyli Zielona Dolina (Babidó1, Poland) as hibernating pupae. Hibernation was broken by storing the pupae at $4{ }^{\circ} \mathrm{C}$ in a cold storage room for five months and another month outdoors. After hibernation, the butterflies were kept in a greenhouse compartment $\left(18 \pm 2{ }^{\circ} \mathrm{C}, 50-60 \% \mathrm{RH}, \mathrm{LD} 16: 8\right)$ with flowering Cardamine hirsuta and Sisymbrium irio plants to obtain eggs. Aglais io butterflies were kept in cages outside (May to June 2018) with cuttings of Urtica sp. plants on which they oviposited. Eggs and/or adults of A. cardamines, Colias spp. and G. rhamni were also collected outdoors (for locations see Table 5.A.2); adults were released again when sufficient egg depositions were obtained. Aporia crataegi eggs were obtained from Worldwide Butterflies, UK (https://www.wwb.co.uk/). Leptidea sinapis eggs were obtained from a population collected in Sweden (Table 5.A.2). P. brassicae, A. crataegi, A. io, M. brassicae and $P$. xylostella lay egg clutches, $P$. napi sometimes lays eggs in small groups, while A. cardamines, Colias spp., G. rhamni, L. sinapis, $P$. mannii and $P$. rapae lay single eggs. 
Methods S2:Preparation of egg wash The concentrations of the egg washes was adjusted based on the size of the eggs used: from 200 eggs per ml for Pieridae to 1000 eggs per $\mathrm{ml}$ for $A$. io (compare database on egg size from more than 10.000 insect species: https://shchurch.github.io/dataviz/index.html). To test the amount of eggs required, we performed an pilot experiment with $B$. nigra and different concentrations of eggs. With an egg wash of 200 eggs per $\mathrm{ml}$ B. nigra consistently responded with strong HR-like response. This was the concentration used to test Pieridae eggs. To wash smaller eggs ( $P$. xylostella, A. io), a concentration of 1000 eggs per ml was used.To screen the Brassicales species, $P$. brassicae wash was made in purified water (Millipore) and Tween 20 was added at a $0.01 \%$ concentration to improve distribution of the egg wash drops onto the waxy leaves of some species. To assess HR-elicitation by other butterfly and moth species, eggs were washed in MES buffer. As controls, paper alone, or Urtica sp. leaves (A. io), a mixture of $C$. hirsuta and $S$. irio inflorescence stems (A. cardamines), leaves of Rhamnus frangula L. (G. rhamni), and inflorescence stems of Iberis spp. (P. mannii), paper (M. brassicae) or parafilm (P. xylostella) were washed in the same manner. Eggs and leaves were kept in the solution overnight, after which the supernatant without eggs was pipetted off and stored at $-20^{\circ} \mathrm{C}$. As these egg washes were tested on B. nigra plants, of which the leaves do not have a wax layer,no Tween20 was added to the washes.

Methods S3: RNA isolation and Real time qPCR analysis of $P R 1$ genes $1 \mu \mathrm{g}$ of RNA was reverse-transcribed into cDNA using Bioline's SensiFAST cDNA synthesis kit (BIO-65054) in a $20 \mu \mathrm{l}$ reaction volume according to the manufacturer's instructions and subsequently diluted $8 \times$ in nuclease free water. Real time qPCR reactions were performed using Bioline's SensiFAST SYBR No-ROX Kit (BIO-98050) in $10 \mu \mathrm{l}$ reaction volumes, containing $3 \mu \mathrm{l} \mathrm{cDNA}$ and $500 \mathrm{~nm}$ of each gene-specific primer (Table 5.A.3) on a CFX96 Touch Real-Time PCR Detection System (Bio-Rad). The following PCR program was used for all PCR reactions: $95^{\circ} \mathrm{C}$ for $2 \mathrm{~min}$ followed by 40 cycles of $95^{\circ} \mathrm{C}$ for $5 \mathrm{~s}$; annealing temperature for $5 \mathrm{~s}$ and $72{ }^{\circ} \mathrm{C}$ for $10 \mathrm{~s}$, with data collection at $72{ }^{\circ} \mathrm{C}$. To check for unspecific PCR products the reactions were followed by a melt curve analysis. $\Delta \Delta \mathrm{Cq}$ values were calculated using the $\mathrm{Cq}$ values of the untreated plants and normalising using the Cq values of the reference genes GAPDH and ACT-2. 
Methods S4: Histochemical staining $\mathrm{H}_{2} \mathrm{O}_{2}$ accumulation was measured at $48 \mathrm{~h}$ with 3,3'-diaminobenzidine (DAB; Sigma) as previously described by Daudi and O'Brien 2012. Cell death accumulation was measured at $72 \mathrm{~h}$ with trypan blue (TB, Sigma) as previously described by Fernández-Bautista et al. 2016. Samples taken at $96 \mathrm{~h}$ were used to assess the egg-induced necrosis by carefully removing the eggs from the leaf disc. Leaf discs with or without eggs and after histochemical staining were imaged with a Dino-Lite digital microscope (AnMo Electronics, Taiwan).Pictures were analysed with Fiji (ImageJ 1.52p) using the plugin Trainable WEKA Segmentation v3.2.34 (Arganda-Carreras et al., 2017) with a customized script to measure the stained or the necrotic leaf area in $\mathrm{mm}$ (Bassetti et al., 2021).

Methods S5: Pieris spp. egg survival assessed by field survey The survey was conducted at a B. nigra patch along the River Rhine in Wageningen (Steenfabriek), The Netherlands (coordinates: $51.96^{\circ} \mathrm{N}, 5.68^{\circ} \mathrm{E}$ ) in one season and one butterfly generation (August-September 2017). The total area monitored was approximately $100 \mathrm{~m}^{2}$ consisting of $\sim 1000$ plants. Plants were monitored for eggs at the edges of a patch or on isolated growing plants so that not all $\sim 1000$ plants were monitored. Leaves with Pieris eggs were collected and checked for the presence of a HR-like necrotic zone on the leaf. After collection, leaves with eggs were kept in Petri dishes in a climate chamber $(25 \pm$ $1{ }^{\circ} \mathrm{C}, 50-70 \% \mathrm{RH}, \mathrm{LD}$ 16:8) until caterpillars emerged. All hatched and dead eggs were recorded.

Methods S6: Phylogenetic tree construction of Brassicales We used the established three-linage classification when planning and conducting our experiments. As some species and genera used in our experiment were not present in neither of the aforementioned studies, we established their relationships with other included species by calculating our own phylogenetic tree using DNA sequences of two chloroplast markers (rbcLand matK) and one nuclear genome marker (ITS2). The sequences were obtained from the BOLD system website (ID numbers see Table 5.A.4) (Ratnasingham and Herbert, 2007). The phylogenetic tree was inferred under maximum likelihood using RaxML v 8.2.4 (GTR+GAMMA, random seed and 1000 bootstrap pseudo-replicates) on the CIPRES science gateway (Miller et al., 2010; Stamatakis, 2014). The three Cleomaceae species were used as outgroups for the phylogenetic tree. 
Chapter 5 Coadaptation of HR-like necrosis

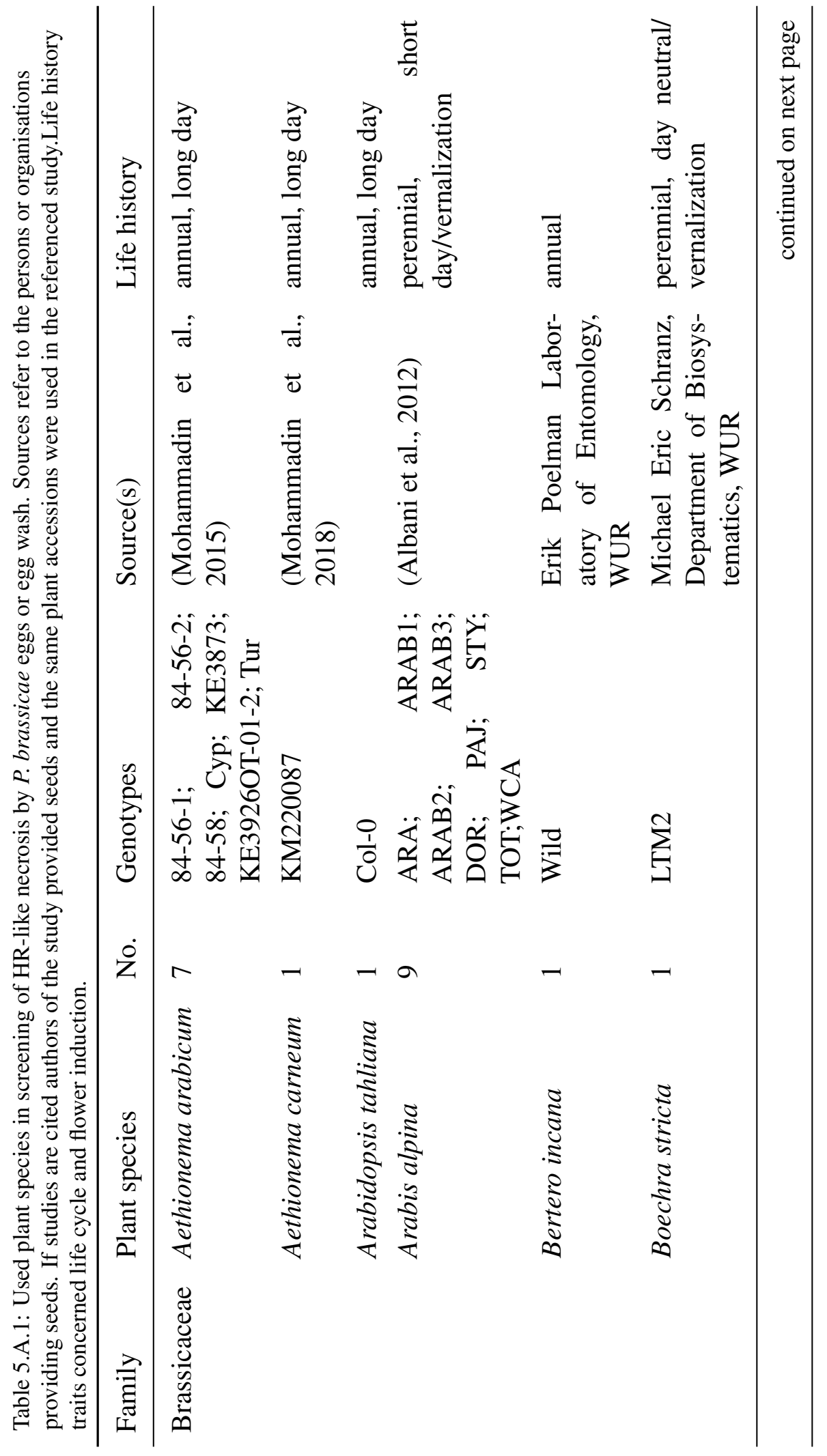




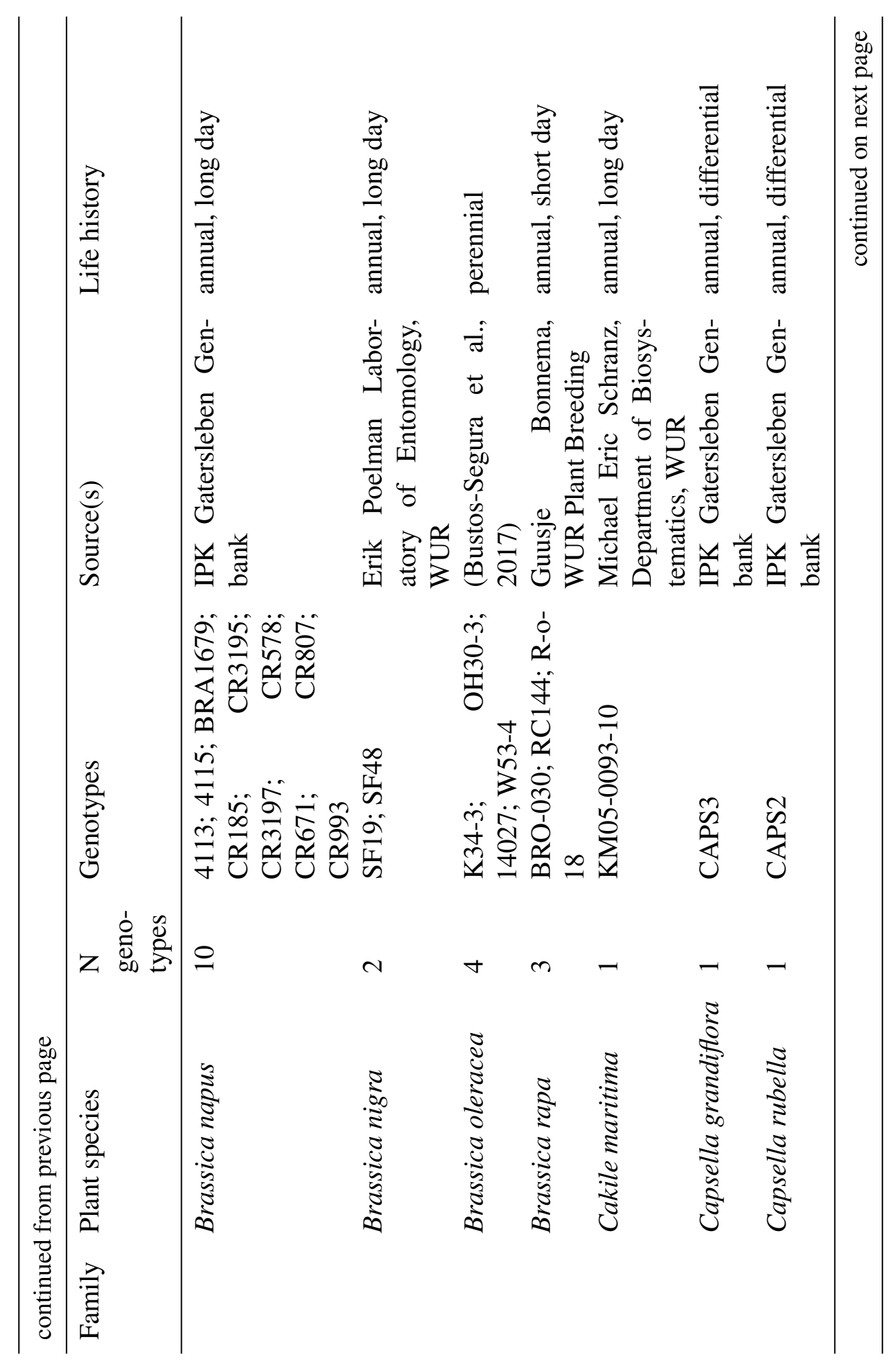


Chapter 5 Coadaptation of HR-like necrosis

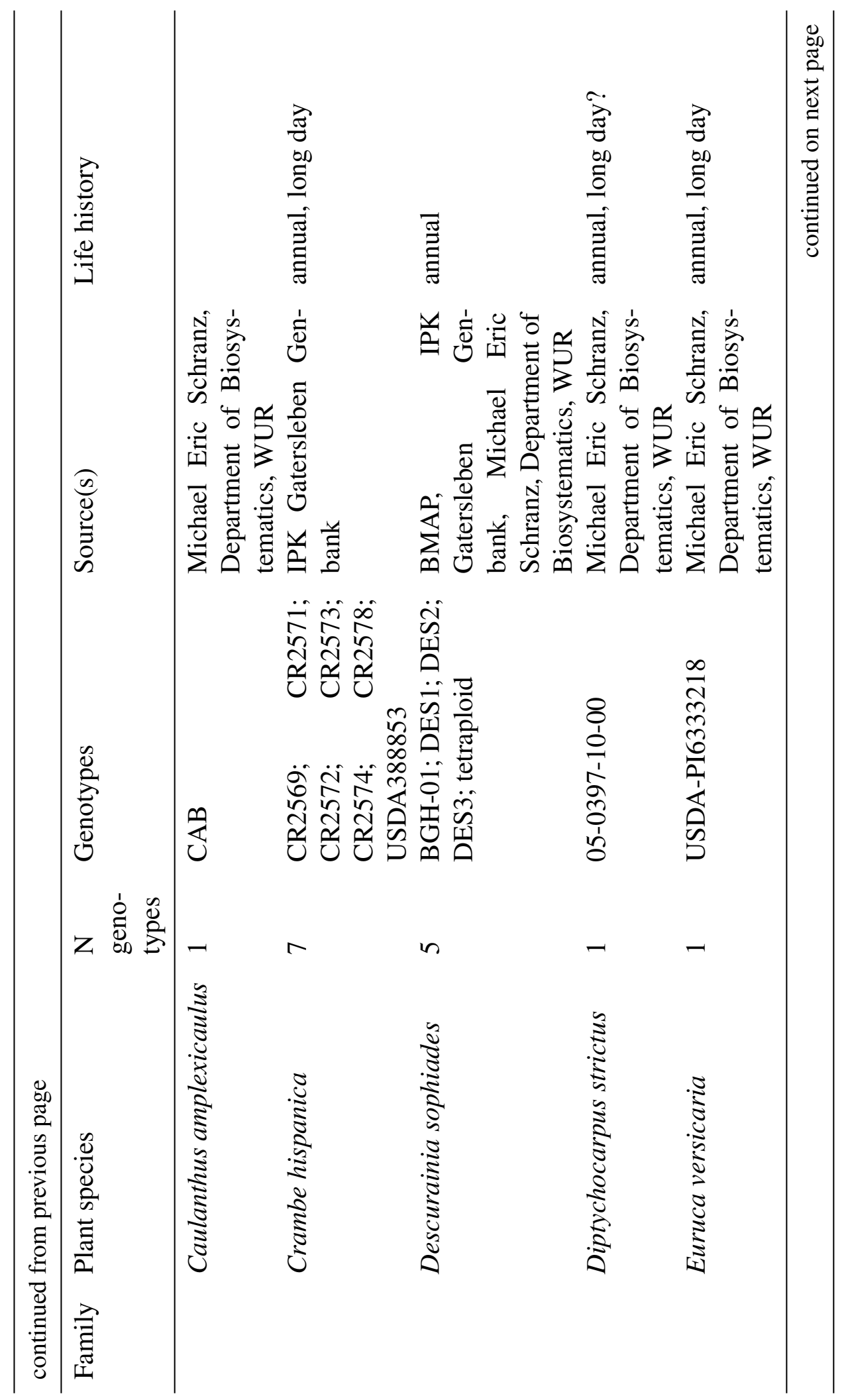




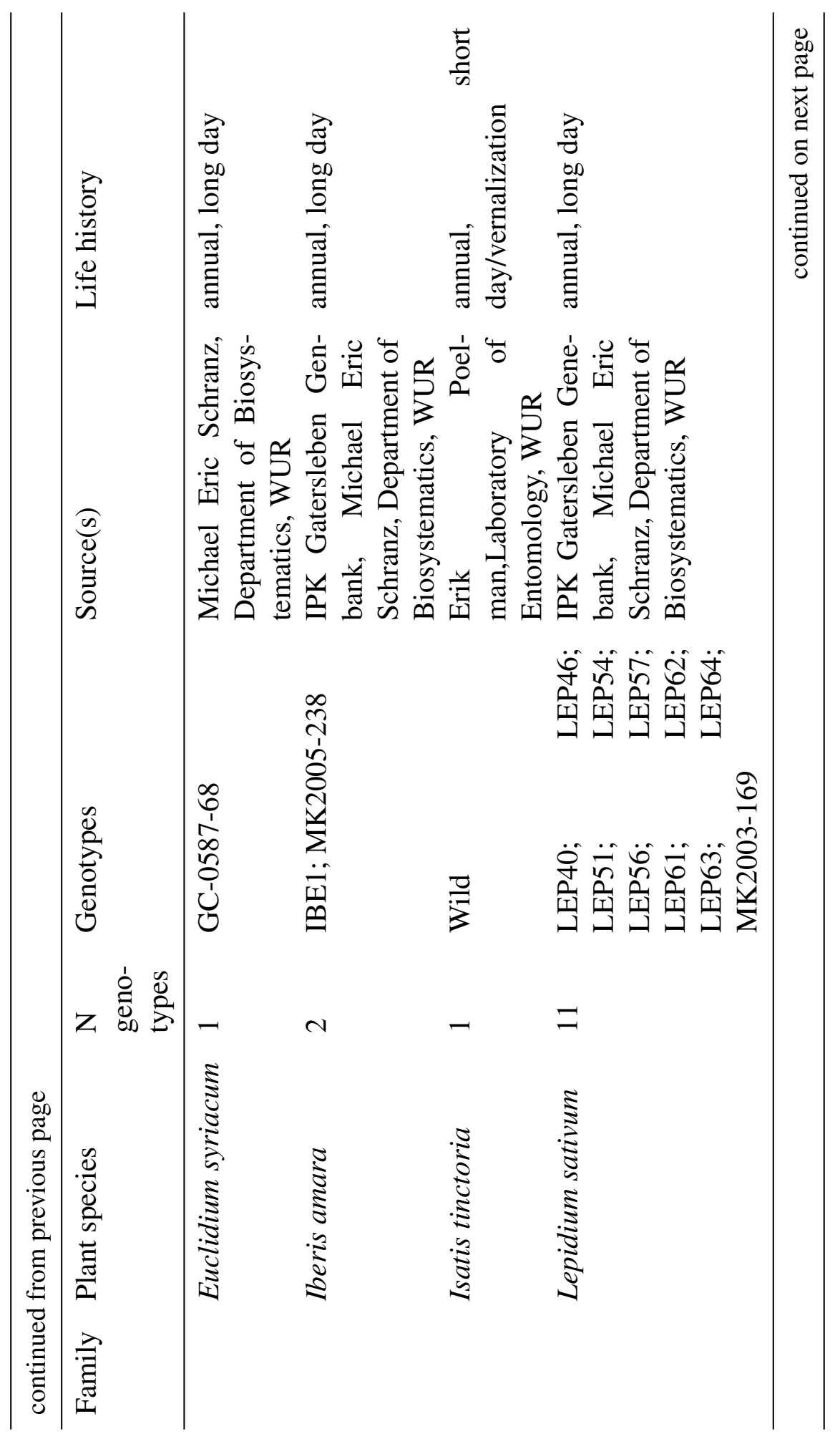




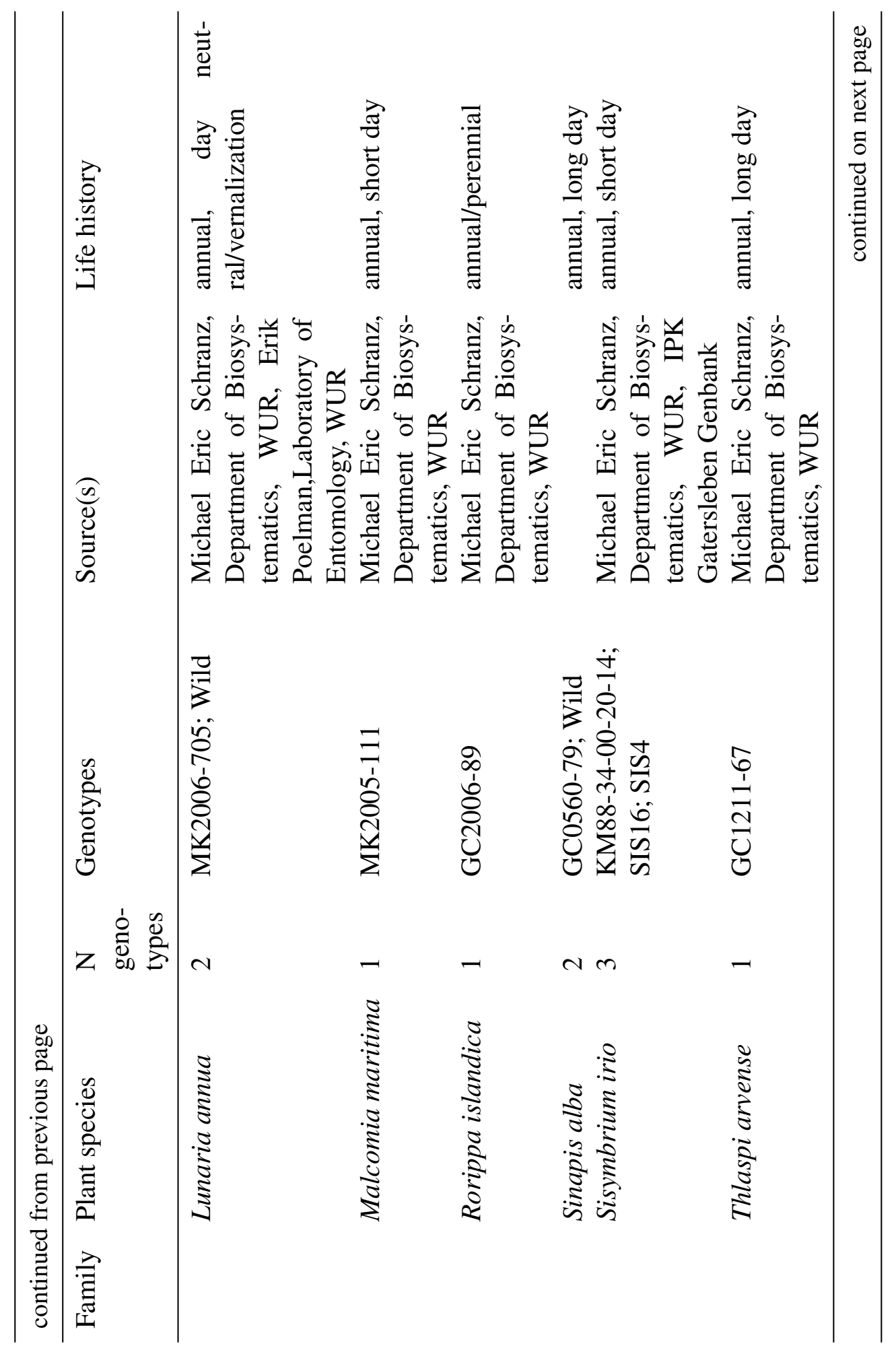




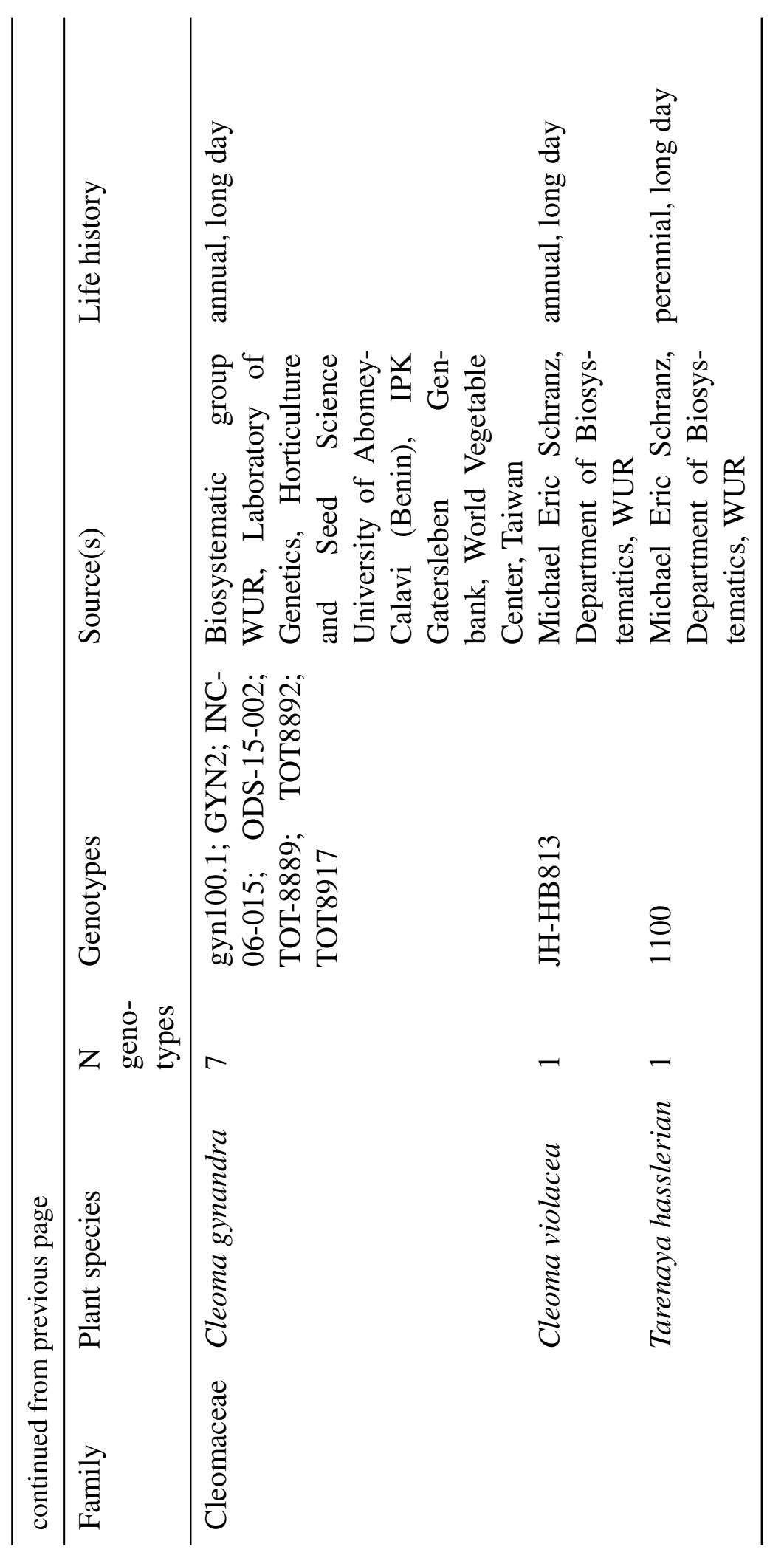


Chapter 5 Coadaptation of HR-like necrosis

Table 5.A.2: Origin of natural and reared butterfly populations used in the study.

\begin{tabular}{|c|c|c|c|}
\hline Butterfly species & Population & Origin & GPS \\
\hline Aglais io & PL & $\begin{array}{l}\text { Butterfly farm in Ba- } \\
\text { bidół }\end{array}$ & $54.2617^{\circ} \mathrm{N}, 18.4418^{\circ} \mathrm{E}$ \\
\hline \multirow[t]{3}{*}{ Anthocharis cardamines } & FR & $\begin{array}{l}\text { Ban-de-Laveline, } \\
\text { Voges }\end{array}$ & $48.2453^{\circ} \mathrm{N}, 7.0661^{\circ} \mathrm{E}$ \\
\hline & FR & Gorges du Segre & $42.4408^{\circ} \mathrm{N}, 2.0803^{\circ} \mathrm{E}$ \\
\hline & PL & $\begin{array}{l}\text { Butterfly farm in Ba- } \\
\text { bidół }\end{array}$ & $54.2617^{\circ} \mathrm{N}, 18.4418^{\circ} \mathrm{E}$ \\
\hline Aporia crataegi & UK & Worldwide Butterflies & \\
\hline Colias sp. & FR & Montgenevre, Alpes & $44.5533^{\circ} \mathrm{N}, 6.4130^{\circ} \mathrm{E}$ \\
\hline Gonepteryx rhamni & NL & $\begin{array}{l}\text { North-East Wagenin- } \\
\text { gen }\end{array}$ & $51.9864^{\circ} \mathrm{N}, 5.6797^{\circ} \mathrm{E}$ \\
\hline Leptidea sinapis & SE & Fiby & $59.5408^{\circ} \mathrm{N}, 17.2131^{\circ} \mathrm{E}$ \\
\hline Mamestra brassicae & NL & $\begin{array}{l}\text { Laboratory of Entomo- } \\
\text { logy, Wageningen Uni- } \\
\text { versity }\end{array}$ & \\
\hline \multirow[t]{2}{*}{ Pieris brassicae } & NL & $\begin{array}{l}\text { Laboratory of Entomo- } \\
\text { logy, Wageningen Uni- } \\
\text { versity }\end{array}$ & $51.9865^{\circ} \mathrm{N}, 5.6634^{\circ} \mathrm{E}$ \\
\hline & PL & $\begin{array}{l}\text { Butterfly farm in Ba- } \\
\text { bidół }\end{array}$ & $54.2617^{\circ} \mathrm{N}, 18.4418^{\circ} \mathrm{E}$ \\
\hline \multirow[t]{2}{*}{ Pieris napi } & NL & $\begin{array}{l}\text { River Rhine, Wagenin- } \\
\text { gen }\end{array}$ & $51.9607^{\circ} \mathrm{N}, 5.6799^{\circ} \mathrm{E}$ \\
\hline & FR & $\begin{array}{l}\text { Estagel, } \quad \text { Pyrenees- } \\
\text { Oriental }\end{array}$ & $42.7724^{\circ} \mathrm{N}, 2.6996^{\circ} \mathrm{E}$ \\
\hline \multirow[t]{2}{*}{ Pieris rapae } & NL & $\begin{array}{l}\text { River Rhine, Wagenin- } \\
\text { gen }\end{array}$ & $51.9607^{\circ} \mathrm{N}, 5.6799^{\circ} \mathrm{E}$ \\
\hline & FR & $\begin{array}{l}\text { Estagel, } \quad \text { Pyrenees- } \\
\text { Oriental }\end{array}$ & $42.7724^{\circ} \mathrm{N}, 2.6996^{\circ} \mathrm{E}$ \\
\hline Pieris mannii & NL & Wageningen & $51.9705^{\circ} \mathrm{N}, 5.6766^{\circ} \mathrm{E}$ \\
\hline Plutella xylostella & NL & $\begin{array}{l}\text { Laboratory of Entomo- } \\
\text { logy, Wageningen Uni- } \\
\text { versity }\end{array}$ & \\
\hline
\end{tabular}


Table 5.A.3: Primer sequences and annealing temperature used in $P R-1$ qPCR

\begin{tabular}{|c|c|c|c|}
\hline Gene & Forward ( $5^{\prime}$ to $\left.3^{\prime}\right)$ & Reverse ( $5^{\prime}$ to $\left.3^{\prime}\right)$ & Annealing Temperature $\left({ }^{\circ} \mathrm{C}\right)$ \\
\hline \multirow[t]{2}{*}{$P R-1$} & CGCCGACGGACTAA & ACACCTCGCTTTGCC & 60 \\
\hline & GAGGCG & ACATCCA & \\
\hline \multirow[t]{2}{*}{$G A P D H$} & GGAGCTGCCAAGGC & CCTTCAGATTCCTCC & 64 \\
\hline & TGTCGG & TTGATAGCC & \\
\hline \multirow[t]{2}{*}{$A C T-2$} & ACATTGTGCTCAGT & TCTGCTGGAATGTGC & 62 \\
\hline & GGTGGA & TGAGG & \\
\hline
\end{tabular}


Chapter 5 Coadaptation of HR-like necrosis

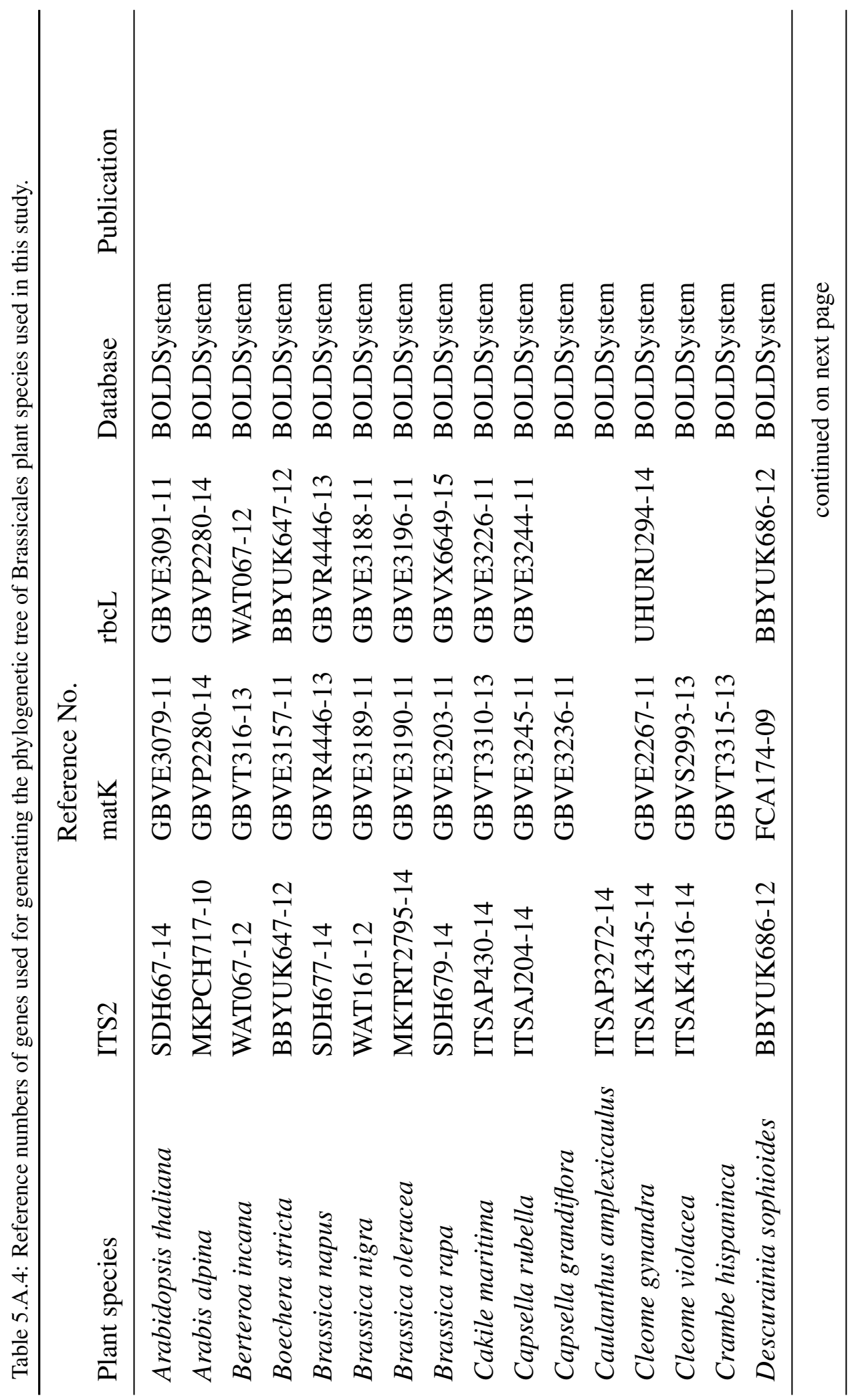




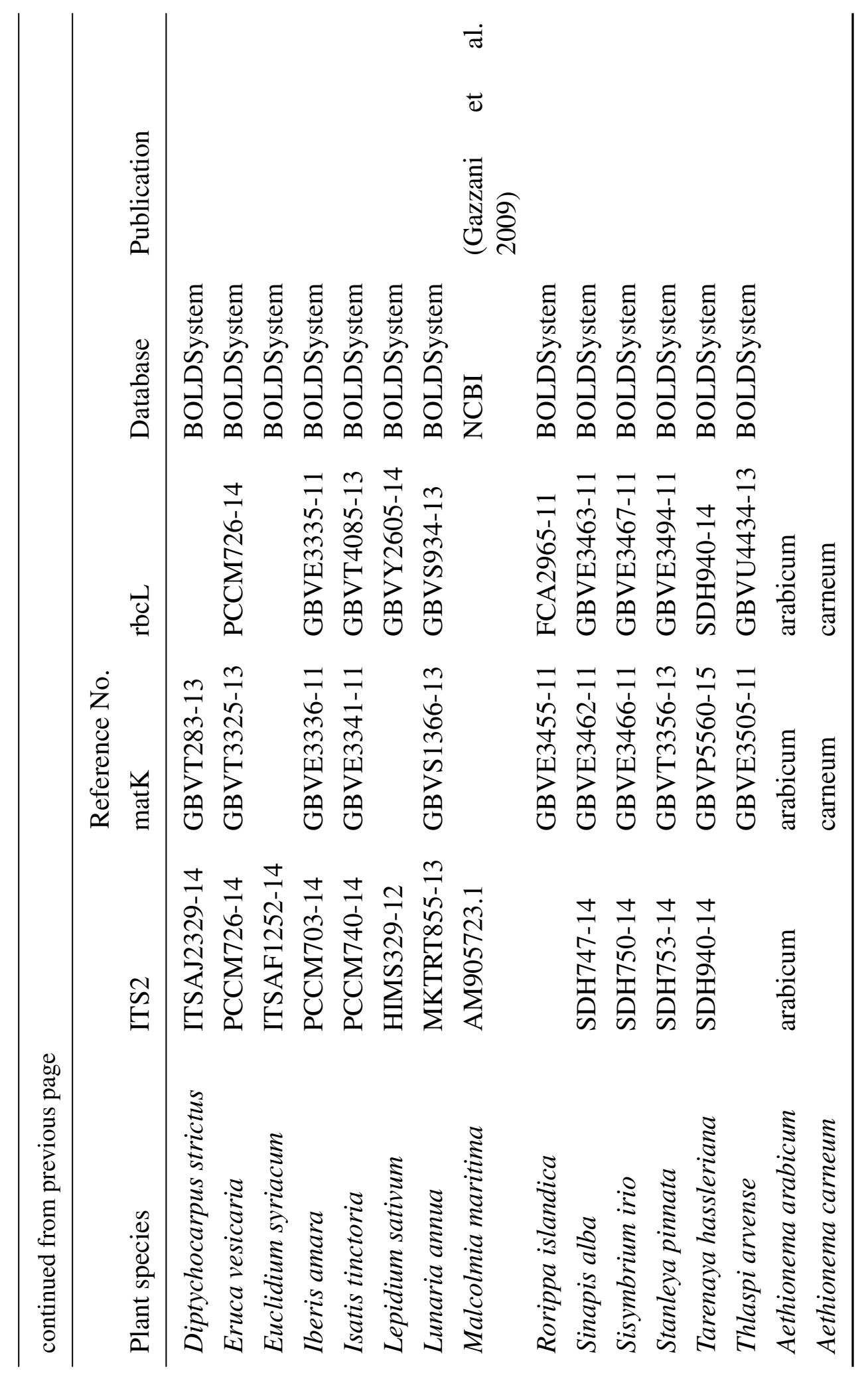


Chapter 5 Coadaptation of HR-like necrosis

Table 5.A.5: Summary of HR-like necrosis induced by $P$. brassicae egg wash in different Brassicaceae and Cleomaceae species. $\mathrm{N}$ indicates the number of treated plants for each species; HR frequency indicates the portion of treated plants showing HR-like necrosis. The order is alphabetical and does not reflect species relationships. $\chi^{2}$ and $P$-values were generated by Chi-square tests $(P<0.05$ in bold $)$.

\begin{tabular}{|c|c|c|c|c|c|}
\hline Plant species & Genotype & $\mathrm{N}$ treated & Fraction HR & $\chi^{2}$ & $P$ \\
\hline \multirow[t]{7}{*}{ Aethionema arabicum } & $84-56-1$ & 6 & 0.33 & 0.6 & 0.44 \\
\hline & $84-56-2$ & 10 & 0.5 & 4.27 & 0.04 \\
\hline & $84-58$ & 15 & 0.6 & 10.16 & 0.001 \\
\hline & Сур & 13 & 0.31 & 2.66 & 0.1 \\
\hline & KE3873 & 5 & 0 & 0 & 1 \\
\hline & KE3926OT-01 & 6 & 0.33 & 0.6 & 0.44 \\
\hline & Tur & 6 & 0.17 & 0 & \\
\hline Aethionema carneum & & 3 & 1 & 2.67 & 0.1 \\
\hline Arabidopsis thaliana & Col-0 & 16 & 0 & 0 & \\
\hline \multirow[t]{9}{*}{ Arabis alpina } & ARA & 9 & 0.11 & 0 & \\
\hline & ARAB1 & 14 & 0.14 & 0.54 & 0.46 \\
\hline & ARAB2 & 15 & 0.07 & 0 & \\
\hline & ARAB3 & 13 & 0.08 & 0 & \\
\hline & DOR & 19 & 0 & 0 & \\
\hline & PAJ & 20 & 0 & 0 & \\
\hline & STY & 16 & 0.1 & 0.002 & 0.96 \\
\hline & TOT & 16 & 0 & 0 & \\
\hline & WCA & 20 & 0 & 0 & \\
\hline Bertero incana & - & 16 & 0.07 & 0 & 1 \\
\hline Boechra stricta & LTM2 & 10 & 0 & 0 & 1 \\
\hline \multirow[t]{3}{*}{ Brassica napus } & 4113 & 7 & 0.86 & 8.14 & 0.004 \\
\hline & 4115 & 14 & 0.43 & 5.3 & 0.02 \\
\hline & BRA1679 & 17 & 0.76 & 17.93 & $<0.001$ \\
\hline
\end{tabular}




\begin{tabular}{|c|c|c|c|c|c|}
\hline \multicolumn{6}{|l|}{ continued from previous page } \\
\hline Plant species & Genotype & $\mathrm{N}$ treated & Fraction HR & $\chi^{2}$ & $P$ \\
\hline & CR185 & 12 & 0.25 & 1.52 & 0.22 \\
\hline & CR3195 & 15 & 0.86 & 18.54 & $<0.001$ \\
\hline & CR3197 & 16 & 0.44 & 6.58 & 0.01 \\
\hline & CR578 & 15 & 0.87 & 19.55 & $<0.001$ \\
\hline & CR671 & 13 & 0.54 & 7.04 & 0.008 \\
\hline & CR807 & 9 & 0.56 & 4.43 & 0.035 \\
\hline & CR993 & 11 & 0.42 & 4.04 & 0.04 \\
\hline \multirow[t]{2}{*}{ Brassica nigra } & SF19 & 8 & 0.63 & 2.4 & 0.12 \\
\hline & SF48 & 6 & 0.83 & 5.49 & 0.02 \\
\hline \multirow[t]{4}{*}{ Brassica oleracea } & $\mathrm{K} 34-3$ & 14 & 0.38 & 4.31 & 0.04 \\
\hline & OH30-3 & 16 & 0.375 & 5.13 & 0.02 \\
\hline & 14027 & 10 & 0.4 & 2.81 & 0.09 \\
\hline & W53-4 & 15 & 0.2 & 1.48 & 0.22 \\
\hline \multirow[t]{2}{*}{ Brassica rapa } & $\mathrm{RC} 144$ & 6 & 0.17 & 0 & \\
\hline & R-o-18 & 13 & 0.15 & 0.54 & 0.46 \\
\hline Cakile maritima & KM05-0093-10 & 3 & 0 & 0 & \\
\hline Capsella grandiflora & CAPS3 & 18 & 0 & 0 & \\
\hline Capsella rubella & CAPS2 & 11 & 0.09 & 0 & \\
\hline Caulanthus amplexicaulus & $\mathrm{CAB}$ & 3 & 0.33 & 0 & \\
\hline \multirow[t]{7}{*}{ Cleome gynandra } & gyn100.1 & 12 & 0.25 & 1.52 & 0.22 \\
\hline & GYN2 & 9 & 0.11 & 0 & \\
\hline & INC-06-01 & 4 & 0.5 & 0.67 & 0.41 \\
\hline & ODS-15-002 & 11 & 0 & 0 & \\
\hline & TOT-8889 & 6 & 0 & 0 & \\
\hline & TOT-8892 & 14 & 0.08 & 0.001 & 0.97 \\
\hline & TOT-8917 & 10 & 0 & 0 & \\
\hline Cleome violaceae & JH-HB813 & 13 & 0.07 & 0.001 & 0.97 \\
\hline Crambe hispanica & CR2569 & 13 & 0.85 & 15.76 & $<0.001$ \\
\hline
\end{tabular}


Chapter 5 Coadaptation of HR-like necrosis

\begin{tabular}{|c|c|c|c|c|c|}
\hline \multicolumn{6}{|c|}{ continued from previous page } \\
\hline Plant species & Genotype & $\mathrm{N}$ treated & Fraction HR & $\chi^{2}$ & $P$ \\
\hline \multirow{10}{*}{ Descurainia sophiades } & CR2571 & 7 & 0.86 & 7.29 & 0.007 \\
\hline & CR2572 & 2 & 0 & 0 & \\
\hline & CR2573 & 14 & 0.57 & 8.58 & 0.003 \\
\hline & CR2574 & 15 & 0.87 & 20.46 & $<0.001$ \\
\hline & CR2578 & 14 & 0.77 & 13.16 & $<0.001$ \\
\hline & USDA388853 & 13 & 0.69 & 10.88 & $<0.001$ \\
\hline & BGH-01 & 15 & 0 & 0 & \\
\hline & DES1 & 16 & 0 & 0 & \\
\hline & DES2 & 6 & 0 & 0 & \\
\hline & DES3 & 16 & 0.06 & 0 & \\
\hline Descurainia tetraploid & - & 8 & 0 & 0 & \\
\hline Diptychocarpus strictus & 05-0397-10-00 & 8 & 0 & 0 & \\
\hline Eruca vesicaria & USDA-PI6333218 & 4 & 0.25 & 0 & \\
\hline Euclidium syriacum & GC-0587-68 & 15 & 0.07 & 0 & \\
\hline \multirow[t]{2}{*}{ Iberis amara } & IBE1 & 13 & 0 & 0 & \\
\hline & IBE11 & 8 & 0 & 0 & \\
\hline Isatis tinctoria & - & 15 & 0.07 & 0 & \\
\hline \multirow[t]{11}{*}{ Lepidium sativum } & LEP40 & 15 & 0 & 0 & \\
\hline & LEP46 & 13 & 0 & 0 & \\
\hline & LEP51 & 16 & 0.06 & 0 & \\
\hline & LEP54 & 13 & 0 & 0 & \\
\hline & LEP56 & 15 & 0 & 0 & \\
\hline & LEP57 & 15 & 0 & 0 & \\
\hline & LEP61 & 14 & 0.06 & 0 & \\
\hline & LEP62 & 14 & 0 & 0 & \\
\hline & LEP63 & 15 & 0 & 0 & \\
\hline & LEP64 & 16 & 0 & 0 & \\
\hline & MK2003-169 & 14 & 0 & 0 & \\
\hline
\end{tabular}


5.A Supplementary material

\begin{tabular}{lccccc}
\hline \multicolumn{1}{l}{ continued from previous page } & \multicolumn{1}{l}{ ( } & & & & \\
\hline Plant species & Genotype & N treated & Fraction HR & $\chi^{2}$ & $P$ \\
\hline Lunaria annua & - & 16 & 0.29 & 3.75 & 0.05 \\
& MK2006-705 & 5 & 0.4 & 0.63 & 0.43 \\
Malcomia maritima & MK2005-111 & 14 & 0.07 & 0 & \\
Rorippa islandica & GC2006-89 & 16 & 0.1 & 0 & \\
Sinapis alba & - & 16 & 0 & 0 & \\
& GC0560-79 & 16 & 0 & 0 & \\
Sisymbrium irio & KM88-34-00-20-14 & 8 & 0 & 0 & \\
& SIS16 & 11 & 0.09 & 0 & \\
Tarenaya hassleriana & SIS4 & 8 & 0 & 0 & \\
Thlaspi arvense & 1100 & 4 & 0 & 0 & \\
\hline
\end{tabular}


Chapter 5 Coadaptation of HR-like necrosis

Table 5.A.6: HR- like necrosis (score ranging from 0 to 3 ) expressed by $B$. nigra plants elicited by different butterfly species. Plants in which the eggs or egg wash tested did induce a HR-like necrosis (HR+) and plants in which they did not (HR-) are counted. SE is given in '()' after values. Different letters indicate significant differences (different when $P<0.025$ ) between butterfly species, Dunn-test, Bonferroni Holm corrected.

\begin{tabular}{lllll}
\hline Butterfly species & HR severity & HR+ & HR- & HR frequency \\
\hline Aglais io & $0(0) \mathrm{b}$ & 0 & 40 & $0(0) \mathrm{b}$ \\
Anthocharis cardamines & $1.63(0.10) \mathrm{a}$ & 61 & 5 & $0.92(0.03) \mathrm{a}$ \\
Colias spp. & $0.67(0.10) \mathrm{ab}$ & 4 & 5 & $0.56(0.18) \mathrm{a}$ \\
Gonepteryx rhamni & $1.11(0.33) \mathrm{a}$ & 8 & 10 & $0.44(0.12) \mathrm{c}$ \\
Pieris brassicae & $1.69(0.13) \mathrm{a}$ & 53 & 12 & $0.82(0.05) \mathrm{a}$ \\
Pieris mannii & $2.14(0.40) \mathrm{ac}$ & 6 & 1 & $0.86(0.14) \mathrm{ac}$ \\
Pieris napi & $2.46(0.16) \mathrm{c}$ & 33 & 4 & $0.89(0.05) \mathrm{a}$ \\
Pieris rapae & $1.64(0.15) \mathrm{a}$ & 42 & 14 & $0.75(0.06) \mathrm{ac}$ \\
\hline
\end{tabular}

Table 5.A.7: HR- like necrosis (score ranging from 0 to 3 ) expressed by $B$. nigra plants elicited by different butterfly and moth species. Shown are mean score and standard error. SE is given in '()' after values. Letters denote differences in HR severity (Wilcoxon, $P<0.05$ ). Number of plants on which the eggs or egg wash was induced a HR-like necrosis (HR+) and plants in which they did not (HR-) were counted and HR frequency calculated. Different letters indicate significant differences between species (EMMEANS test).

\begin{tabular}{lllll}
\hline Butterfly species & HR severity & HR+ & HR- & HR frequency \\
\hline Anthocharis cardamines & $1.55(0.04) \mathrm{b}$ & 13 & 7 & $0.65 \mathrm{ab}$ \\
Aporia crataegi & $1.10(0.05) \mathrm{bc}$ & 11 & 9 & $0.55 \mathrm{ab}$ \\
Colias spp. & $0.60(0.04) \mathrm{cd}$ & 6 & 14 & $0.30 \mathrm{~b}$ \\
Gonepteryx rhamni & $0.31(0.03) \mathrm{d}$ & 2 & 17 & $0.11 \mathrm{~b}$ \\
Leptidea sinapis & $0.70(0.10) \mathrm{bcd}$ & 2 & 8 & $0.20 \mathrm{~b}$ \\
Mamestra brassicae & $0.36(0.04) \mathrm{d}$ & 3 & 16 & $0.16 \mathrm{~b}$ \\
Pieris mannii & $2.95(0.01) \mathrm{a}$ & 18 & 0 & $1.00 \mathrm{a}$ \\
Pieris rapae & $2.88(0.01) \mathrm{a}$ & 18 & 0 & $1.00 \mathrm{a}$ \\
Plutella xylostella & $0.00(0.00) \mathrm{d}$ & 0 & 6 & $0.00 \mathrm{~b}$ \\
Control (MES buffer) & $0.21(0.03) \mathrm{d}$ & 2 & 19 & $0.10 \mathrm{~b}$ \\
\hline
\end{tabular}




\section{A Supplementary material}

Table 5.A.8: Relative expression of $P R l$ gene upon treatment with egg wash of different butterflies/moths and MES buffer as control. $\mathrm{N}$ indicates biological replicates (average of 4 pooled plants), $P R 1$ relative expression is represented by median \pm SE. Letters denote differences in mean transcript levels between different treatments (Kruskal-Wallis rank sum test followed by Dunn's multiple comparison test, $P<0.05$, performed on $\log$-transformed data).

\begin{tabular}{lll}
\hline Butterfly species & $\mathrm{N}$ & PRI relative expression \\
\hline Anthocharis cardamines & 5 & $328.9 \pm 51.6 \mathrm{~b}$ \\
Aporia crataegi & 5 & $27.9 \pm 9.3 \mathrm{bc}$ \\
Colias spp. & 4 & $19.44 \pm 7.6 \mathrm{~cd}$ \\
Gonepteryx rhamni & 5 & $5.86 \pm 12.6 \mathrm{c}$ \\
Mamestra brassicae & 5 & $5.04 \pm 0.9 \mathrm{~d}$ \\
Pieris mannii & 4 & $2134.2 \pm 239.1 \mathrm{a}$ \\
Pieris rapae & 5 & $1229.3 \pm 159.5 \mathrm{a}$ \\
Plutella xylostella & 4 & $15.6 \pm 7.3 \mathrm{~d}$ \\
Control (MES buffer) & 5 & $1.1 \pm 0.39 \mathrm{e}$ \\
\hline
\end{tabular}

Table 5.A.9: Quantification of HR-like necrosis in B. nigra, B. oleracea and C. hispanica leaves upon oviposition of $P$. brassicae egg clutches (10 eggs). Mean \pm standard error (SE) of HR-like necrosis in $\mathrm{mm}$ is presented below. Single data points are presented in Figure 5.A.2. Letters denote differences in mean HR-like necrotic area (ANOVA followed by Tukey post-hoc test with Benjamini-Hochberg correction, $P<0.05)$.

\begin{tabular}{lll}
\hline Butterfly species & $\mathrm{N}$ & $P R 1$ relative expression \\
\hline Anthocharis cardamines & 5 & $328.9 \pm 51.6 \mathrm{~b}$ \\
Aporia crataegi & 5 & $27.9 \pm 9.3 \mathrm{bc}$ \\
Colias spp. & 4 & $19.44 \pm 7.6 \mathrm{~cd}$ \\
Gonepteryx rhamni & 5 & $5.86 \pm 12.6 \mathrm{c}$ \\
Mamestra brassicae & 5 & $5.04 \pm 0.9 \mathrm{~d}$ \\
Pieris mannii & 4 & $2134.2 \pm 239.1 \mathrm{a}$ \\
Pieris rapae & 5 & $1229.3 \pm 159.5 \mathrm{a}$ \\
Plutella xylostella & 4 & $15.6 \pm 7.3 \mathrm{~d}$ \\
Control (MES buffer) & 5 & $1.1 \pm 0.39 \mathrm{e}$ \\
\hline
\end{tabular}




\section{Chapter 5 Coadaptation of HR-like necrosis}
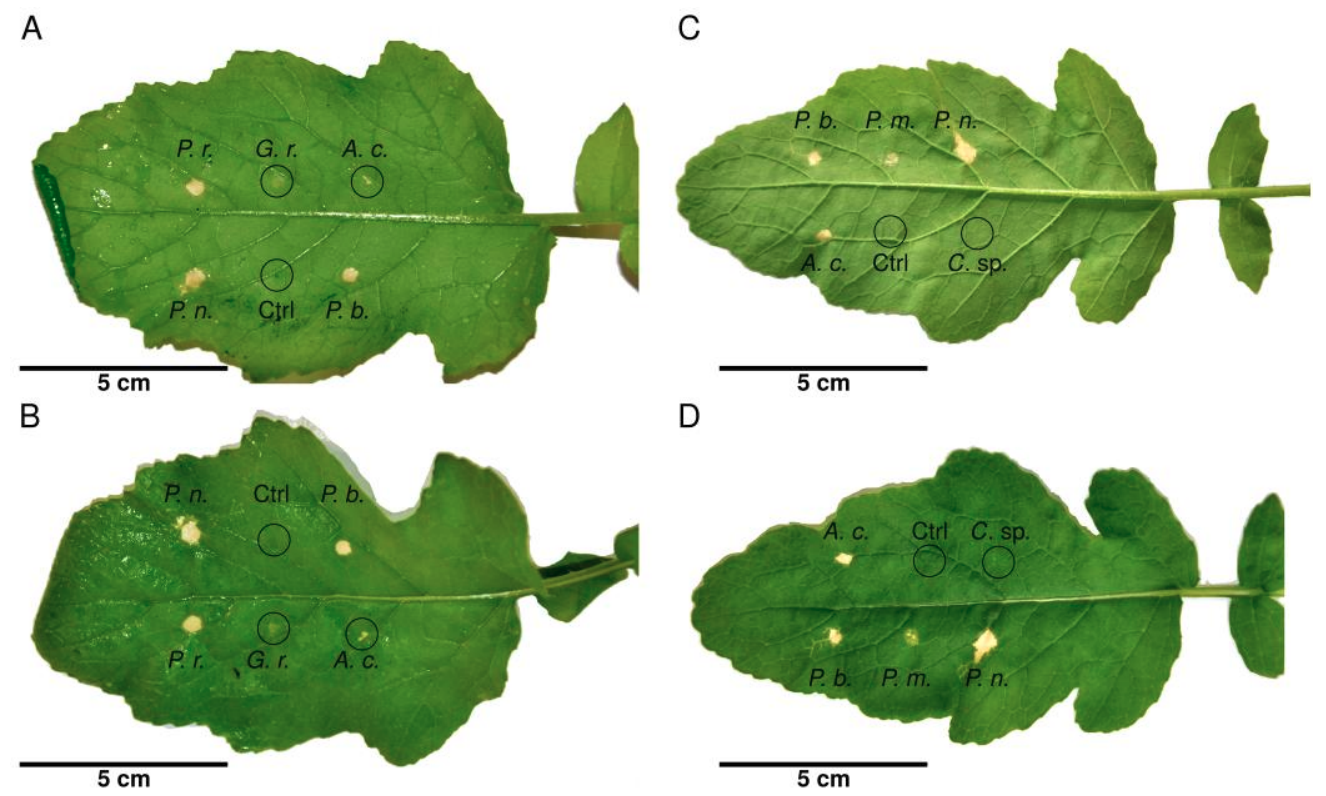

Figure 5.A.1: Leaves from B. nigra treated with egg wash of different butterfly species and controls inducing or not a HR-like necrosis. Pieris brassicae (P. b.), P. mannii, (P. m.), P. napi (P. n.), and $P$. rapae (P. r.) and A. cardamines (A. c.) induce a strong HR-like necrosis. Egg wash of G. rhamni (G. r.) and Colias sp. (C. sp.) induces a very faint response resembling a chlorosis and does not fit into the established scoring system (faintness indicates 1 but showing up on both sides of the leaf indicates 2). The control (buffer without eggs) does not elicit a HR-like necrosis. All egg washes had the same concentration (200 eggs $\mathrm{ml}^{-1}$ ) and amount applied onto the leaf ( $\left.5 \mu \mathrm{l}\right)$. Two leaves were needed as not all egg washes were available at the same time. A) and C) Abaxial side of the leaf where the egg washes were applied onto. B) and D) Adaxial side of the leaf showing how strong the HR-like response is on the side which was not treated with egg wash. 


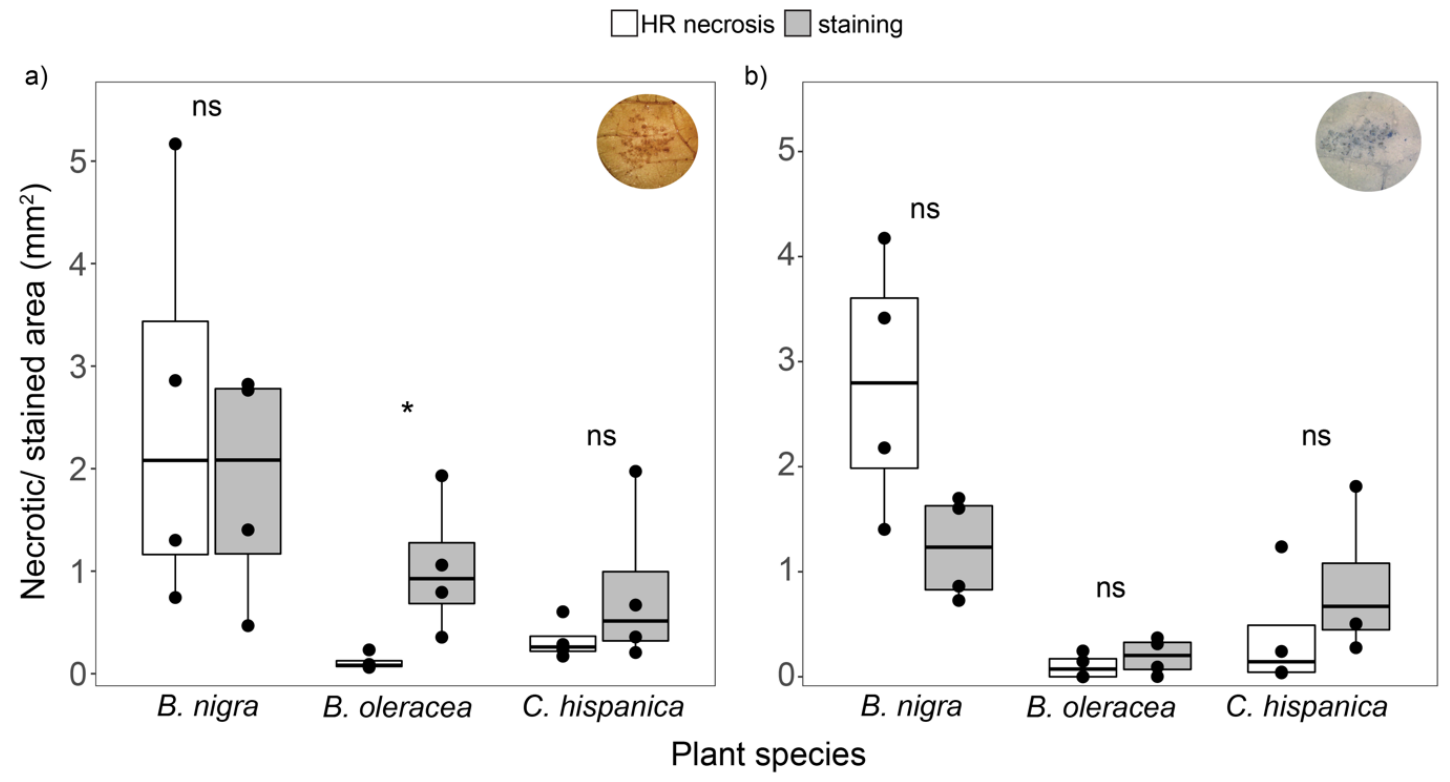

Figure 5.A.2: Quantification of HR necrosis and histochemical staining of reactive oxygen species and cell death in B. nigra, B. oleracea and C. hispanica leaves upon oviposition of $P$. brassicae egg clutches. A) $\mathrm{H}_{2} \mathrm{O}_{2}$ by DAB staining, b) cell death by TB staining. A representative picture of the staining is presented on the top right corner of each graph. Boxplots depicts $1^{\text {st }}, 3^{\text {rd }}$ quantile and median, each dot represents a plant treated with an egg clutch (10 eggs). For each plant species $\mathrm{N}=$ 4 plants were used for both experiments. Asterisks denote statistically significant difference between HR necrosis and histochemical staining (Student's t-test, ns: not significant, $* P<0.05$ ). 
Chapter 6

General Discussion

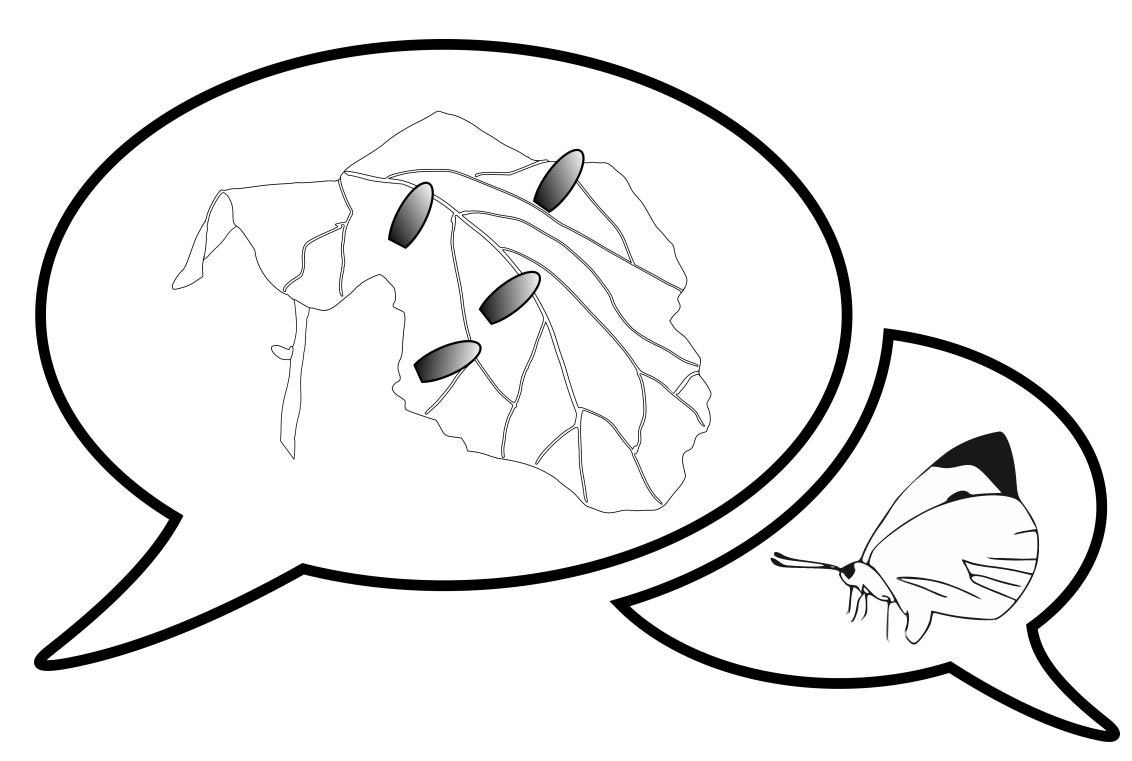

155 


\section{Introduction}

The coevolutionary arms race between plants and insects has led to the development of sophisticated plant defence and insect counter-defence strategies (Ehrlich and Raven, 1964; Futuyma and Agrawal, 2009). It has been suggested that the evolution of diverse and new constitutive secondary defence metabolites at the dawn of angiosperm evolution has contributed to the success of flowering plants, which form the foundation of most terrestrial ecosystems (Kubitzki and Gottlieb, 1984; Reimann et al., 2004). However, herbivorous arthropods have counteradapted in various ways such as by evolving detoxifying enzymes to facilitate feeding (Gatehouse, 2002). Plants have responded and enhanced their defences by evolving more sophisticated defence strategies, such as induced rather than constitutively expressed chemical defences. Induced defences can be more finely tuned by the plant to deliver the optimal defence strategy when under attack by herbivores, while also limiting the use of resources (e.g. improved resource allocation). Furthermore, induced defences allow plants to produce the appropriate defence only when specifically needed (Zangerl, 2003). An induction of defences is especially important when plant defences are only effective against certain herbivores.

Different plant species use different defence strategies and even genotypes within a species might differ in defences expressed, to maximize their fitness (Cipollini et al., 2017). Specific plant species or plant genotypes can induce a balanced array of defensive traits, such as specific mixtures of toxins and other chemicals, specifically detrimental for specific herbivores (Dicke and Hilker, 2003; Heil and Karban, 2010). While the mixture of constitutive and induced chemical defences may already present a well rounded sophisticated anti-herbivore defence, plants and herbivores do not exist alone in the environment. Predators and parasitoids will search for and feed on herbivores, and plants are able to recruit them in their own defensive struggles (Dicke and Sabelis, 1988; Heil, 2008; Price et al., 1980).

The aforementioned arms race between plants and herbivores is now understood to also include interactions between plants and the predators and parasitoids of the herbivores. For their multi-layered defences, plants attract, arrest and reward the enemies of herbivores. There is a variety of such indirect defences. Beneficial arthropods that defend the plant can be arrested by the food provided by extrafloral nectaries. Some plants can also provide shelter for their defenders (e.g. domatia of Acacia trees for ants (Heil 
and McKey, 2003)). Intimate interactions between plants and defenders will mainly attract generalistic predators that can deal with a variety of herbivores.

Other more targeted approaches also exist. For example, plants can "call for help" upon herbivore attack by releasing volatile blends that inform natural enemies about the presence of herbivores. Specific blends can also carry information about the specific species of herbivore, therefore specialist predators and parasitoids can be enlisted for plant defence (McCormick et al., 2012). Especially parasitoids are often specialised to attack only specific developmental stages of herbivores, which limits the window of time for attack. An underappreciated but fascinating case of recognizing and defending against a particular developmental stage is the detection of an insect egg by plants.

Significant evidence now exists of direct and indirect egg-induced defences being ubiquitous and that they can lower egg survival rates (Hilker and Fatouros, 2015; Fatouros et al., 2016). Egg-induced responses are recorded for gymnosperms (Bittner et al., 2017; Hilker and Meiners, 2002) and most angiosperm clades (Fatouros et al., 2016). To date, there is not a coevolutionary perspective involving insect eggs and egg-induced plant responses, especially macroevolutionary studies similar to those on secondary metabolites and feeding stages of herbivores (Edger et al., 2015; Wheat et al., 2007). Therefore, as of now it is unknown whether and how egg-killing defences shape both plant- and insect populations over time.

\section{Aim of the thesis and special note}

The aim and originality of my thesis was to elaborate on the ecology and evolution of plant responses to oviposition and which role egg killing might have played in the continuous arms race between plants and specialist herbivorous insects. To the best of my knowledge, it was the first time that a macroevolutionary approach was used, to indicate coevolution between butterfly eggs and their host plants. Not only did I show that some egg-killing responses evolved in plants, but also the potential counteradaptations of herbivorous insects to egg killing. To understand the role of egg-induced responses in the evolution of host-plant selection, I evaluated their effect on herbivore preferences and performances, and their effects on plant fitness. I examined the existence of natural variation in egg-induced plant responses within a plant species, and how it affects not only the survival of eggs and caterpillar performance on the plants but also how the 


\section{Chapter 6 General Discussion}

plant responses influence the interaction with natural enemies of herbivores and conspecific herbivore females. Finally, I looked at the prevalence of egg-killing necrosis within a plant family (Brassicaceae) and their elicitation of egg-induced responses by different pierid butterflies. A nymphalid butterfly (closely related to Pieridae but not feeding on Brassicaceae) and two moth species (distantly related to Pieridae but feeding on Brassicaceae) were added as outgroups. I used both laboratory and (semi-) field studies to conduct my research.

The main questions of my thesis were: (1) How effective is the hypersensitive response (HR)-like necrosis against eggs of the gregarious Pieris brassicae (Chapter 2)? (2) Does variation in egg-induced responses exist within a plant species (Chapters 2 and 3) and how does this affect different trophic levels (Chapter 3)? I extended the number of plant species and added a second Pieris butterfly species to understand (3) how egg deposition influences preference and performance of a gregarious and a solitary Pieris species (Chapter 4)? And finally, I expanded the collection of plants and butterflies even more to answer (4) how widespread is HR-like necrosis among the Brassicaceae and eggs of which butterfly species can induce it (Chapter 5)? With the results of this thesis, our understanding of the role insect eggs play in insect-plant coevolution has been expanded.

Note, that in two chapters, 2 and 3, I am hinting at a common-garden field experiment conducted in two consecutive years (2013 and 2014). In both chapters, the results gathered from the field take a small portion within the story of the paper. This could lead to the mistaken impression that the field experiments were relatively small set-ups, easily done within a short period of time. However, in reality for both years, the set-up of the field, the monitoring and data analysis took much of my time and effort. The initial study question of the common-garden trials was to test whether egg-induced responses, both direct and indirect, contribute to plant fitness. Sadly, the results gathered from the field did in the end suffer from diluting effects of biotic and abiotic noise. I found small effects of egg parasitoids that were less abundant than assumed, and HR-like necrosis had no effect on the survival of clustered eggs. Since the treatments and plant expression of necrosis did not lead to different outcomes for herbivores, no differences in plant fitness could be found and therefore the field work added only minor contributions to insights obtained by this thesis. Field work will always be a valuable tool especially in ecological research. That my common-garden experiment conducted over two summer 
seasons did not fulfil expectations is disappointing, but at least this experience can be used as valuable experience for future field experiments being planned.

Below I link the results of my thesis with the literature on the subject. Finally, I speculate on future directions my research can inspire.

\section{Egg-killing a new counteradaptation in the arms race?}

While toxic secondary metabolites evolved to protect plants from generalist insect herbivores, specialist herbivores evolved counteradaptations, like genes encoding detoxifying enzymes (Brattsten, 1991; Wittstock et al., 2004). As herbivores overcome plant defences over time, selection pressure can bring forth plants with more, better, or novel defences. In other words, the arms race between plants and herbivores further escalates.

The addition of more diverse glucosinolates in the Brassicaceae can be seen as an element of such an arms race escalation (Edger et al., 2015). In some rare cases plants can recruit a new class of chemical compounds into their defensive toolkit. The plant genus Erysimum (Brassicaceae) evolved - next to the expected glucosinolates - cardenolides as toxic metabolites (Jaretzky and Wilcke, 1932; Kowalewski et al., 1960) (Figure 6.1). The novel gain of cardenolides may deter many glucosinolates-adapted Brassicaceae specialist herbivores from feeding on Erysimum plants, even if more generalist herbivores still feed on them (Züst et al., 2020). To date no specialist feeding on Erysimum plants has been found, and especially Brassicaceae specialists do not feed on them (Züst et al., 2020). While diversified chemical defences can be quite helpful in deterring herbivores, limited resources make it difficult for plants to defend against all possible herbivores. Expressing a defensive trait will come with costs in other areas such as plant growth and ultimately fitness. However, measuring the costs of defensive traits can be quite difficult especially in complex environments (Agrawal, 2011). 

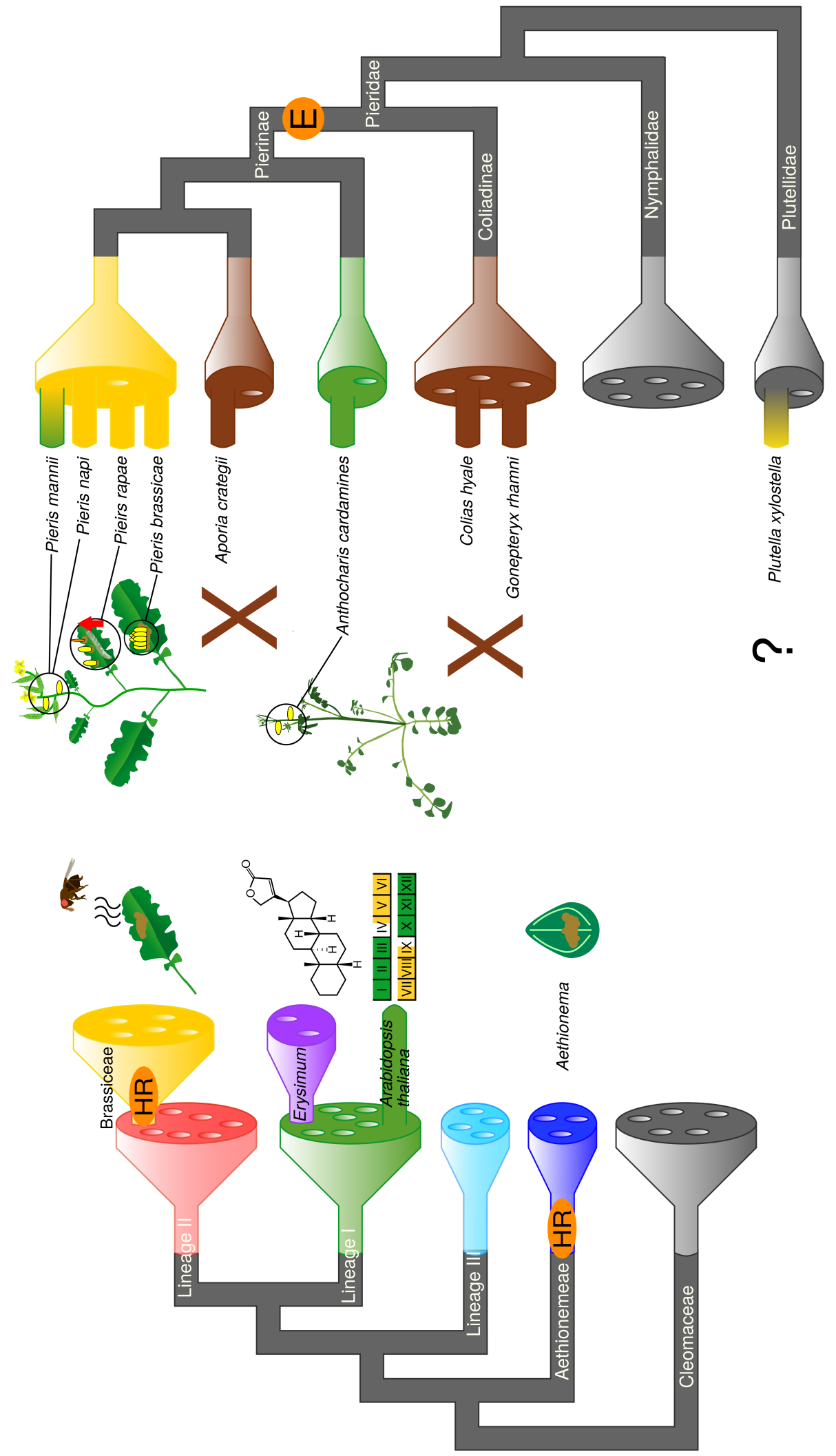

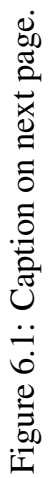


Figure 6.1: (Previous page.) Proposed coevolutionary scheme between Brassicaceae plants and Pieridae butterfly eggs. All Brassicaceae plants are to some extent protected from generalist herbivores by the glucosinolate-myrosinase system. The colours of the clades indicate an herbivore-host plant relation (co-diversification) and help to point out special adaptations and counteradaptations. Some adaptations on the plant side further prevented specialist caterpillar feeding. The genus Erysimum evolved cardenolides as additional defensive compounds (Züst et al., 2020). Some plants, e.g. A. thaliana escaped phenologically from herbivory, meaning their lifecycles do generally not overlap with the butterfly's lifecycle. Besides phenological escape and plant toxins, some Brassicaceae plants evolved egg-killing defences. The production of volatiles upon oviposition to attract egg parasitoids has been shown for three Brassica plant species of the Brassiceae tribe (Afentoulis et al., 2021; Fatouros et al., 2012) and was shown to vary within plant species (Chapter 3), while HR-like necrosis against specialist Pieridae eggs evolved at least twice in the Brassicaceae, in the tribes Aethionemeae and Brassiceae (indicated by HR on the phylogram) (Chapter 5). This necrosis is specifically elicited by an elicitor found in the egg glue of species from the subfamily Pierinae (indicated by E on the phylogram) (Chapter 5). On the herbivore side, several counteradaptations to plant defences evolved. As specialist herbivores, some Lepidoptera caterpillars adapted to the glucosinolates-myrosinase system by evolving NSP enzymes (Pieridae) or sulfatase (Plutella xylostella). Butterflies of the genus Aporia do not feed on Brassicaceae but Rosaceae, therefore circumventing Brassicaceae specific defences. Potentially to counteract oviposition-induced defences, $A$. cardamines and $P$. mannii are both specialized to feed on Lineage I Brassicaceae, of which tested plant species did not express HR-like necrosis (chapter 5). Together with $P$. napi, A. cardamines also oviposit onto the inflorescent stem of their host plants where no HR-like necrosis is expressed (E. Griese, N.E. Fatouros, personal observations). Pieris brassicae lays egg clusters, an oviposition mode, which prevents egg killing by HR-like necrosis (Chapter 2). Pieris rapae does not seem to have evolved counteradaptations to egg-killing necrosis. However, $P$. rapae caterpillars showed an increased larval performance on plants expressing HR-like necrosis (indicated by the red arrow next to the $P$. rapae caterpillar) (Chapter 4). It is not known whether $P$. xylostella has evolved any counteradaptations to egg-induced defences of Brassicaceae (Chapter 5).

Similar to anti-feeding chemical defences, a plethora of anti-egg defences exist in plants. Since only the 1980s, we know that responses to insect eggs are part of the defensive toolbox of plants. Over time, different types of egg-killing responses have been reported in many different plant families, see Figure 6.2, reviewed by Bertea et al. (2020), Fatouros et al. (2016), Hilker and Fatouros (2015) and Hilker and Fatouros (2016). Some egg-killing traits have so far only been reported for single plant species, e.g. the production of ovicidal substances and the gummosis sealing (rice (Oryza sativa) (Seino et al., 1996) and cherry (Prunus serotina) (Karban, 1983), respectively). Other egg killing responses are more widespread over the plant kingdom. Neoplasm formation has been reported both in Fabaceae and Solanaceae (Doss et al., 1995; Doss et al., 2000; Geuss et al., 2017; Petzold-Maxwell et al., 2011). Wound tissue growth has been reported in the Apoxaceae, Myrtaceae and Lauraceae (Aluja et al., 2004; Desurmont and Weston, 2011; Desurmont et al., 2021; Mazanec, 1985). Finally HR-like necrosis 


\section{Chapter 6 General Discussion}

(or chlorosis with the release of reactive oxygen species) is reportedly widespread in the plant kingdom. Fabaceae (Garza et al., 2001; Baruha et al., 2017; Das et al., 2021), Brassicaceae (Fatouros et al., 2014; Gouhier-Darimont et al., 2019; Griese et al., 2021; Griese et al., 2020; Griese et al., 2017; Little et al., 2007; Shapiro and DeVay, 1987), Myrtaceae (although no egg-killing has been reported) (Oates et al., 2021), Solanaceae (Balbyshev and Lorenzen, 1997; Geuss et al., 2017; Petzold-Maxwell et al., 2011), Apocynaceae (Kalske et al., 2014), the monocotyledon family Poaceae (Yang et al., 2014), and even in the gymnosperm family Pinaceae (Bittner et al., 2017). Interestingly, the examples are mostly limited to crop plants or closely related species. This sampling bias may leave high potential for more plant species with potentially new egg-killing traits to be discovered. Direct egg-killing responses are mostly employed against specialist herbivores, which can be assumed to be able to cope with anti-feeding plant defences (Figure 6.2).

At the same time insect counteradaptations to those egg-induced defences have been described (Desurmont and Weston, 2011; Griese et al., 2017). Therefore, I speculate that a similar escalating arms race between anti-egg defences and insect eggs/gravid females has evolved. 
Egg-killing defences

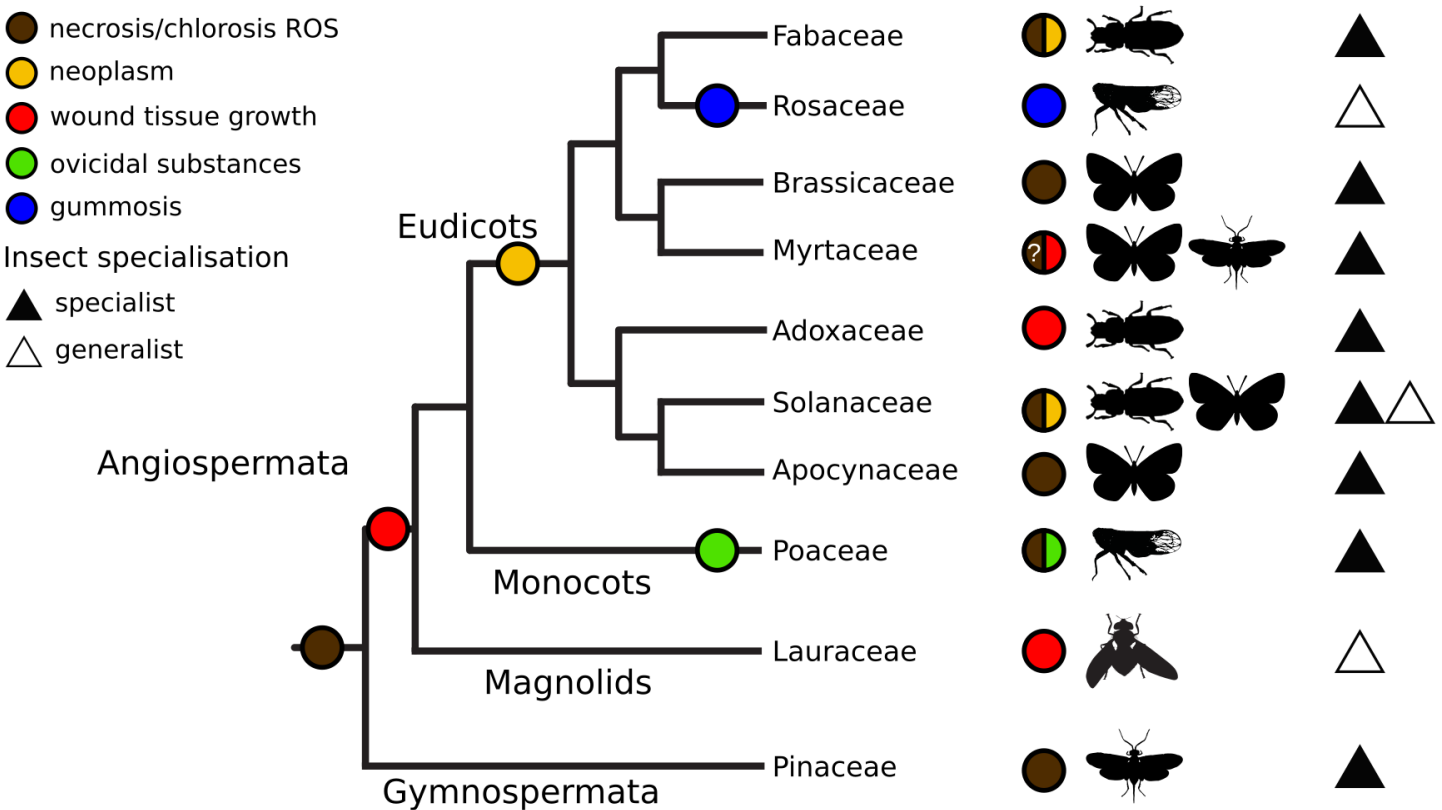

Figure 6.2: Direct egg-killing defences are widespread in the plant kingdom. The phylogenetic tree shows only plant families for which egg-killing defences could be found. The coloured circles on the tree indicate one possible origin of each egg-killing trait. However, the small sample size of species per plant family makes it hard to conclude anything definitive. Insect orders against which a direct egg-killing trait has been observed are shown as silhouettes. Broadly there does not seem to be a relation between the plant families and insect orders which they respond to. However, the egg-killing responses are mainly expressed against specialist herbivores (filled triangles). The '?' for the Myrtaceae necrosis indicates that while the release of reactive oxygen species has been shown, no egg killing has been observed so far. 


\section{Herbivore counteradaptations to egg-induced plant defences}

For some anti-feeding arms races, the genetic basis on both sides (plants and herbivores) has been established. Brassicaceae use as main toxic secondary metabolites glucosinolates. Upon leaf damage, hydrolysis of glucosinolates by the enzyme myrosinase leads to toxic compounds such as nitriles, thiocyanates or isothiocyanates. Two specialist detoxification mechanisms and the enzymes used for the purpose have recently been described. Pieris butterflies detoxify hydrolysis products of glucosinolates using nitrile-specifier proteins (NSPs) (Wittstock et al., 2004). Plutella moths on the other hand change the chemical structure of glucosinolates, making them "unrecognizable" for myrosinase, using glucosinolate sulfatase (Heidel-Fischer and Vogel, 2015; Ratzka et al., 2002).

Armed with the knowledge of the genetic basis for the glucosinolate-myrosinase system and the insect counterpart of NSPs, the arms race between the butterfly family Pieridae and the plant order Brassicales has been beautifully shown in a macroevolutionary context (Wheat et al., 2007; Edger et al., 2015). The macroevolutionary aspects of coevolution have long been ignored (Futuyma and Agrawal, 2009). In the PieridaeBrassicales arms race the introduction of different and more complex glucosinolates in the Brassicales and the evolution of different NSPs in the Pieridae could both be linked to a shift in speciation rates (Wheat et al., 2007; Edger et al., 2015).

To demonstrate the existence of an evolutionary arms race between insect eggs and egg-killing plant defences, as proposed in my thesis, more research is needed to validate this hypothesis, which still is in its very early stage. So far, the phenotypic existence of traits such as the HR-like necrosis and potential counteradaptations of Pierinae butterflies was demonstrated. Which receptor(s) and elicitor contribute to the trait is currently investigated, and this will shed more light on the understanding of the evolution of insect egg-induced HR-like necrosis. In Chapter 5 of this thesis, I showed that HR-like necrosis is largely expressed in two groups of the Brassicaceae, in the tribe Brassiceae and for the genus Aethionema. While those two groups are phylogenetically distantly related in the Brassicaceae (Al-Shehbaz, 2012; Guo et al., 2017; Huang et al., 2015), both are described as host plants for Pieris spp. (Réal et al., 1967; Fatouros et al., 2014; Shapiro and DeVay, 1987; Friberg et al., 2015; Fei et al., 2014). The genus Pieris and a closely related genus Anthocharis were also the only butterflies tested to elicit 
a strong HR-like necrosis on B. nigra plants. This two-sided specificity indicates that this defence evolved specifically against brassicaceous-feeding pierid butterflies. These specialist butterflies adapted to the glucosinolate defence and are even able to fine-tune their NSP expression to the glucosinolate content of their host plant (Okamura et al., 2019). Therefore, it might not be surprising that plants evolved different (egg-induced) defences (Figure 6.1). Ehrlich and Raven (1964) proposed their idea of coevolution based on the known plant toxins and butterfly taxa adapted to the plant taxa and their toxins. Similarly, egg-induced defences and possible counteradaptations could have led to co-diversification between butterflies and plants.

A specific elicitor only found in Pierinae egg secretions seems to induce the eggkilling response in B. nigra plants (Caarls et al., 2021; Griese et al., 2021). Although, I cannot be sure if the other butterflies tested would elicit necrosis on their main host plants, I could not find any literature on necrotic responses to Pieridae eggs laid on Rosaceae, Fabaceae or Rhamnaceae, and both my own and other observations confirm this (Personal observation, N. E. Fatouros).

The elicitor was found to be present in the female accessory reproductive glands (ARGs). A homogenates of ARGs from mated P. brassicae females did elicit plant responses in B. oleracea (Fatouros et al., 2015; Caarls et al., 2021). In general, the ARG provides protein-rich secretions, the egg glue, which envelops the egg surface and helps the butterfly to glue the egg onto the leaf surface. As such, the composition of the ARG secretion will differ with the needs of the insect egg. Besides being used to glue eggs to surfaces, egg secretions can provide anti-microbial protection and/or transmit symbionts (Marchini et al., 1991; Flórez et al., 2017; Paniagua Voirol et al., 2018). Since egg-induced HR-like necrosis is similar to a pathogen-induced HR, it was hypothesized that bacteria transferred in the ARG secretion elicit the necrosis beneath the eggs (Fatouros, personal communication). However, the low number of bacteria found in Pieris ARG secretions makes this unlikely (Paniagua Voirol et al., 2020). Chemical analysis of the egg glue that dissolves in water and keeps its eliciting function after heating and freezing, hint to a chemical (or mixture of chemicals) that is neither a lipid nor a protein (Caarls, Bassetti, Fatouros et al., unpublished data, Caarls et al. 2021). Recently, phosphatidylcholines (PCs) were shown to play a role in $P$. brassicae egg-induced responses in A. thaliana (Stahl et al., 2020). These chemicals are part of cell membranes and therefore not specific to Pierinae butterflies. Therefore, because of the elicitors spe- 
cificity to Pieris and Anthocharis eggs, I suggest that it is a chemical compound, which the Pierinae developed after the split from the rest of the Pieridae.

Species of the other Pieridae subfamilies, like the Coliadinae and Dismorphinae did not elicit a necrosis. With our data it is hard to conclusively reason if the expression of HR-like necrosis in response to insect eggs is ancestral in the Brassicaceae or evolved convergently, at least twice, in the Brassicaceae. While eggs of Aporia butterflies did not elicit a response similar to eggs of Pieris butterflies, shifting to Rosaceae as a host plant could have led to a loss in the elicitor. For example, in the way that the egg glue might have had to adapt to be used on leaf surfaces of Rosaceae plants. The elicitor for HR-like necrosis and other oviposition-induced plant responses is most likely produced by the butterflies themselves and once found, will make further research possible.

HR-like necrosis seems to be especially widespread in the plant kingdom as an eggkilling response. Many studies are observations and often effects on the egg survival are not recorded or measured. Survival rates of Heliothis subflexa moth eggs on Physalis spp. leaves were lowered by approximately $10 \%$ due to HR-like necrosis (PetzoldMaxwell et al., 2011). Furthermore, the survival of Pieris spp. eggs can be reduced by up to $60 \%$ on plants expressing HR-like necrosis (chapter 5), and when including eggs being parasitized by parasitoids that are more attracted to plants expression HRlike necrosis at the oviposition site, this reduction in survival can even increase up to $80 \%$ (Fatouros et al., 2014).

It is obvious from my thesis and previous research that HR-like necrosis can lower egg survival (Shapiro and DeVay, 1987; Petzold-Maxwell et al., 2011; Fatouros et al., 2014; Griese et al., 2017). Therefore, the question arises why butterfly eggs elicit such negative responses? Why is the elicitor still present if it harms the embryos that much? While there are no satisfying explanations yet, it can be speculated that the butterflies simply have no choice. In order to increase their own reproductive success female insects must evolve ways to circumvent or lower the effect of egg-induced plant defences. Consequently, it is likely that different counteradaptations against egg-killing plant responses have evolved. For the Pierinae butterflies three main counter-adaptations to egg-killing plant responses have been proposed: Egg clustering (Figure 6.3a), oviposition on inflorescences (Figure 6.3b) and a host plant shift (Figure 6.3c). 


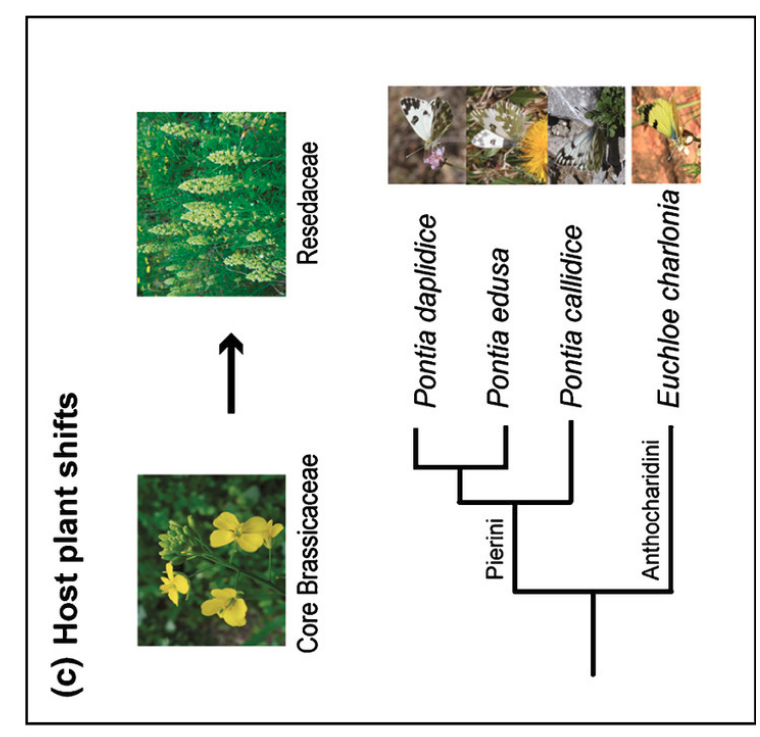

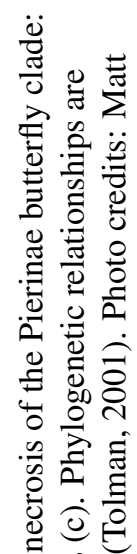

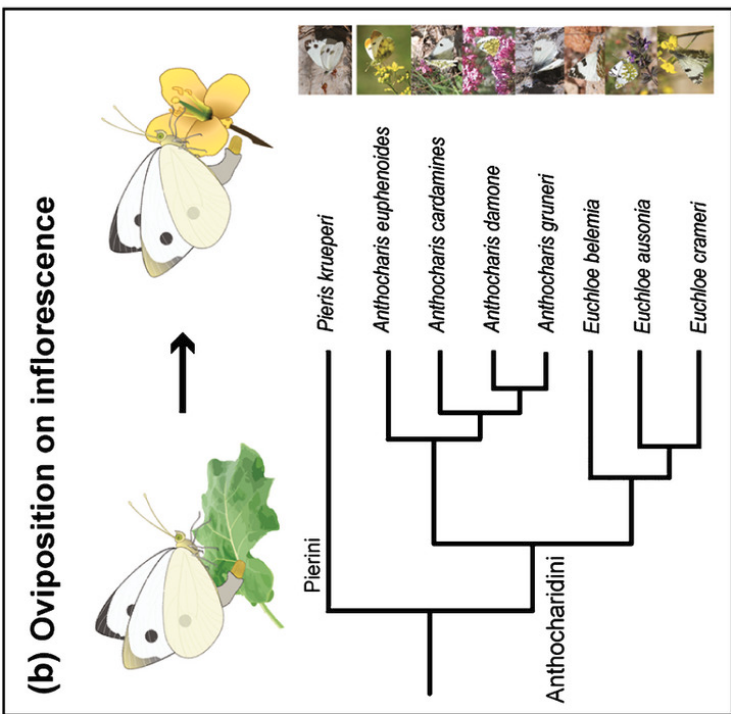

ช.

के के

荬

范芯芯

造造

.

.

刓

总氖产

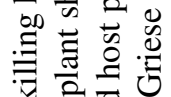

品旅

\& 용

음

.0ิ

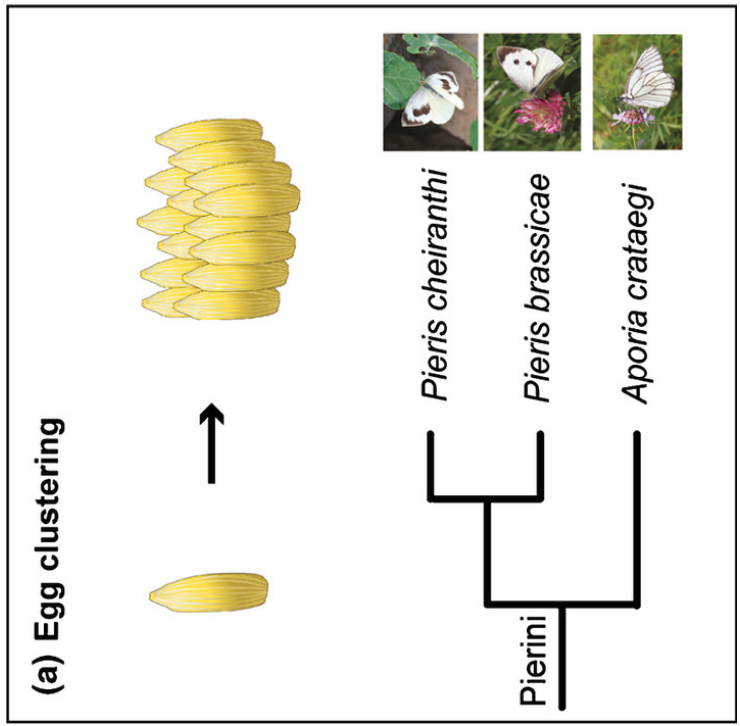

䒕 00.

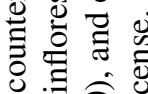

능ำ

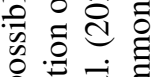

पू.

के क्षे

芯芯氞

II

$\ddot{\theta}$ ส

ชั

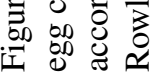




\section{Gregarious egg-laying as a counteradaptation to HR-like necrosis}

In general, several different advantages of gregariousness in butterflies have been discussed (Stamp, 1980), but it seems that different selection pressures act in different butterfly groups. For several butterfly species advantages for gregariously feeding larvae have been shown. Hatching larvae feeding gregariously have an advantage compared to artificially singly feeding larvae. Gregariously living nettle-feeding nymphalid butterflies are able to raise their body temperature higher in sunlight compared to solitary living ones (Bryant et al., 2000). A commonly held hypothesis is that aposematic coloured larvae lead to the evolution of gregarious larvae and egg clustering (SillénTullberg, 1988). While the discussion around this topic is still ongoing, it seems in general that most advantages proposed for gregariousness are found in the larval stage.

Protection from egg desiccation provides an alternative explanation for the origin and maintenance of egg clustering in Lepidoptera and other insects (Stamp, 1980). Testing the egg-desiccation hypothesis, Clark and Faeth (1998) studied the oviposition strategy of the nymphalid butterfly Chlosyne lacinia. They measured survival of varying egg batch sizes and egg-layering arrangements on the common sunflower (Helianthus annuus) under controlled humidity levels. Both, eggs laid in larger numbers and with greater number of layers were shown to survive better under low humidity conditions than smaller, monolayered egg batches (Clark and Faeth, 1998).

In my thesis, I show for the first time that clustering eggs counteracts the egg-killing effect of HR-like necrosis (Chapter 2, (Griese et al., 2017)). I compared the survival of ten $P$. brassicae eggs laid as a small clutch with the survival of 10 singly laid $P$. brassicae eggs. I show that $P$. brassicae eggs laid in clusters do not suffer higher mortality on plants expressing HR-like necrosis. This begs the question, why are not more butterflies using this strategy?

The reason for that could be that clustering eggs also has trade-offs. Gregariously laid eggs and hatching caterpillars can invite cannibalism. Egg clustering also helps the spread of infectious diseases and may provide an "all you can eat buffet" to parasitoids and predators (Myers and Rothman, 1995). Some egg parasitoids hitchhike on gravid females to get access to newly laid eggs (Clausen, 1976; Huigens and Fatouros, 2013). This strategy is especially rewarding if many eggs can be found in close proximity. Furthermore, some plant defences seem to be enhanced with larger numbers of eggs per 
plant, which is why some insects avoid oviposition on plants laden with conspecific eggs (Bruce et al., 2010; Fatouros and Huigens, 2012; Meiners et al., 2005). These negative consequences of egg clustering might partly explain why most butterfly species refrain from it.

\section{Other ways to counteract egg-killing by plants}

Besides egg clustering, other ways to avoiding negative consequences of egg-killing might have evolved, like oviposition on inflorescence or shifts to host plants that did not evolve HR-like necrosis. As shown in Figure 6.3b, some pierid butterflies, especially in the Anthocharini oviposit on inflorescence instead of the leaves. This enables the hatching caterpillars to easily feed from the resource- and glucosinolate-rich flowers. More importantly in the context of counter adaptations to plant defences, so far HR-like necrosis has not yet been observed on inflorescence stems. This adds another benefit to laying eggs on flower stems.

Other pierid butterflies, mainly in the genus Pontia and Euchloe might have undergone a host shift away from the Brassicaceae plants, which express HR-like necrosis, towards the Resedaceae, for which such an egg-killing response has not been demonstrated. Furthermore, some Pieris butterflies might have specialised on Brassicaceae plants, which do not express HR-like necrosis, such as Pieris mannii that feeds on Iberis spp., which is not part of lineage II (Beilstein et al., 2006; Nikolov et al., 2019).

So far, mainly $P$. rapae has been reported to suffer from egg mortality due to HR-like necrosis under natural egg-laying conditions (Shapiro and DeVay, 1987; Fatouros et al., 2014; Griese et al., 2021) (Chapter 3). In Chapter 3, I not only show that egg survival of $P$. rapae is reduced by HR-like necrosis under greenhouse conditions, but the butterflies actually preferred to lay eggs on plants with a high likelihood of expressing HR-like necrosis, thus reducing the performance of their offspring.

According to the preference-performance hypothesis (PPH) or the 'mother-knowsbest hypothesis', ovipositing females should maximize their fitness by selecting plants on which offspring survival will be high, thus female preferences reflect offspring performances (Jaenike, 1990). Evidence in support of this hypothesis is controversial and a number of ecological and/or life-history factors have been proposed to modify the strength of this preference-performance relationship (Berenbaum and Feeny, 1981; 


\section{Chapter 6 General Discussion}

Knolhoff and Heckel, 2014). In a meta-analysis, Gripenberg et al. (2010) showed support for the PPH when herbivore diet breath was taken into account: oligophagous herbivores prefer good-quality plants for their offspring more than polyphagous insects. However, other variables tested, such as egg distribution patterns, feeding by adults or offspring mobility did not influence a positive link between preference and performance. Hilker and Fatouros (2015) suggest that the PPH might be more often supported when studies would include the most often neglected questions how the egg stage performs and how plant-mediated effects of eggs affect offspring performances.

For $P$. rapae the negative effect that HR-like necrosis has on eggs seems to be offset by the caterpillars feeding on plants expressing necrosis, where they gain more weight after 7 days. Thus, I argue that in general (and certainly for the example of $P$. rapae) the counteradaptation to egg-killing plant responses could be increased fitness of larvae feeding on especially those plants killing eggs.

\section{Conclusions and future directions}

Egg-induced responses of plants are a vital part of the plants defensive toolkit and have for a long time been underappreciated and understudied. Recently, more attention has been paid to the ways in which recognition and responses to insect eggs may help plants to better defend against insect attackers. The research presented in this thesis builds upon previous research on egg-induced plant defences and helps to place it in a more evolutionary context. It sheds new light on the interactions between specialist butterfly eggs such as P. brassicae and Brassicaceae plants.

My results suggest that variation of egg-induced defences exist within plant species (Chapter 2, Chapter 3 and Chapter 5) and that this variation (due to differences in volatiles released) affects behavioural changes of different trophic levels (Chapter 3 and Chapter 4). This indicates that there is some trait variation within natural populations on which natural selection can work. Furthermore, the results demonstrate that HR-like necrosis, as an egg-killing trait, is clade-specific for Brassicaceae and its elicitation is highly specific for Pierinae butterflies (Chapter 5). These results together with evidence for a counteradaptation against egg-induced plant defences (Chapter 2) point towards an ongoing arms race between host plants and specialist insect eggs.

While the results of my thesis do not clearly show an effect of oviposition-induced 
defences on plant fitness in the system studied, other studies show that ovipositioninduced responses increase seed production (Pashalidou et al., 2015b; Pashalidou et al., 2020). Egg-induced defences could potentially play an important role in crop protection (Fatouros et al., 2016; Tamiru et al., 2015). HR-like necrosis has been demonstrated to be effective in reducing the number of hatching caterpillars, especially under field conditions (Griese et al., 2021; Fatouros et al., 2014). This is promising, as crops such as cabbage, rape seed and mustard that are part of the lineage II in the Brassicaceae express HR-like necrosis. Interestingly, in combination with OIPVs a double defence exists for some Brassica plants, HR-like necrosis expressing plants also attract more egg parasitoids (Afentoulis et al., 2021; Fatouros et al., 2014). A recent genome-wide association analysis of stemborer egg-induced plant volatiles in maize identified markers that might help breeders to select for indirect defence in maize crops (Tamiru et al., 2020).

In a similar fashion the identification of the, as of yet, unknown elicitor located in or on the insect egg will help further research on egg-induced responses. Light could be shed on the receptor-genes for the elicitor in plants. On the insect side genes involved in the production of the elicitor could be identified, as well as answering what biological function the elicitor may serve for the insect. Using egg wash, which avoids getting the contents of the egg mixed with the compounds on the leaf surface, the elicitor is currently identified using HPLC and mass spectrometry. So far, a candidate class of compounds for the elicitor is terpenes (Caarls, Bassetti, Fatouros et al., unpublished data). Terpenes in general are produced by both plants and insects for various uses (Beran et al., 2019). Candidate elicitor genes might encode enzymes of the terpene synthase (TPS) gene family in the nymphalid Heliconius melpomene with mono- and sesquiterpene synthase activities, and seem to have arisen independently from those in other insect clades. Screening a large number of Lepidoptera species for copies of these genes could then be used to improve the phylogenetic understanding of the origin of the elicitor.

This research also brings up the question what the genetic mechanism is behind phenotypic differences in egg-induced HR-like necrosis. Recently, candidate genes were uncovered which explain differences in plant defence inducibility by insect eggs (Groux et al., 2020; Tamiru et al., 2020). For A. thaliana, it has been shown that a lectin receptor kinase LecRK-I.8 is involved in the recognition of insect eggs (Gouhier-Darimont et al., 
2013).

As more and more Brassica genomes become available, including those of $B$. rapa and B. nigra (Perumal et al., 2020), finding responsive and unresponsive genotypes of a plant species can be used as a first step to understanding the genetic basis for specific egg-induced defences, such as HR-like necrosis. Increasing the number of plant genomes screened for fitting receptor genes will make it possible to zoom in on the origin of the egg-killing necrosis or even to the origin of egg-induced responses in general. Adding screenings on elicitor-producing genes in the insects, a macroevolutionary picture of the arms race involving insect eggs will deliver a clearer picture beyond the phenotypic one of Chapter 5 (Griese et al., 2021). Looking at egg-induced responses and counteradaptations this thesis is a starting point of understanding egg-induced responses in plants in an arms race scenario. As such it can inspire future research on macroevolutionary questions as well as on in-depth understanding of the molecular basis for insect egg-plant interactions. 
Chapter 7

Bibliography

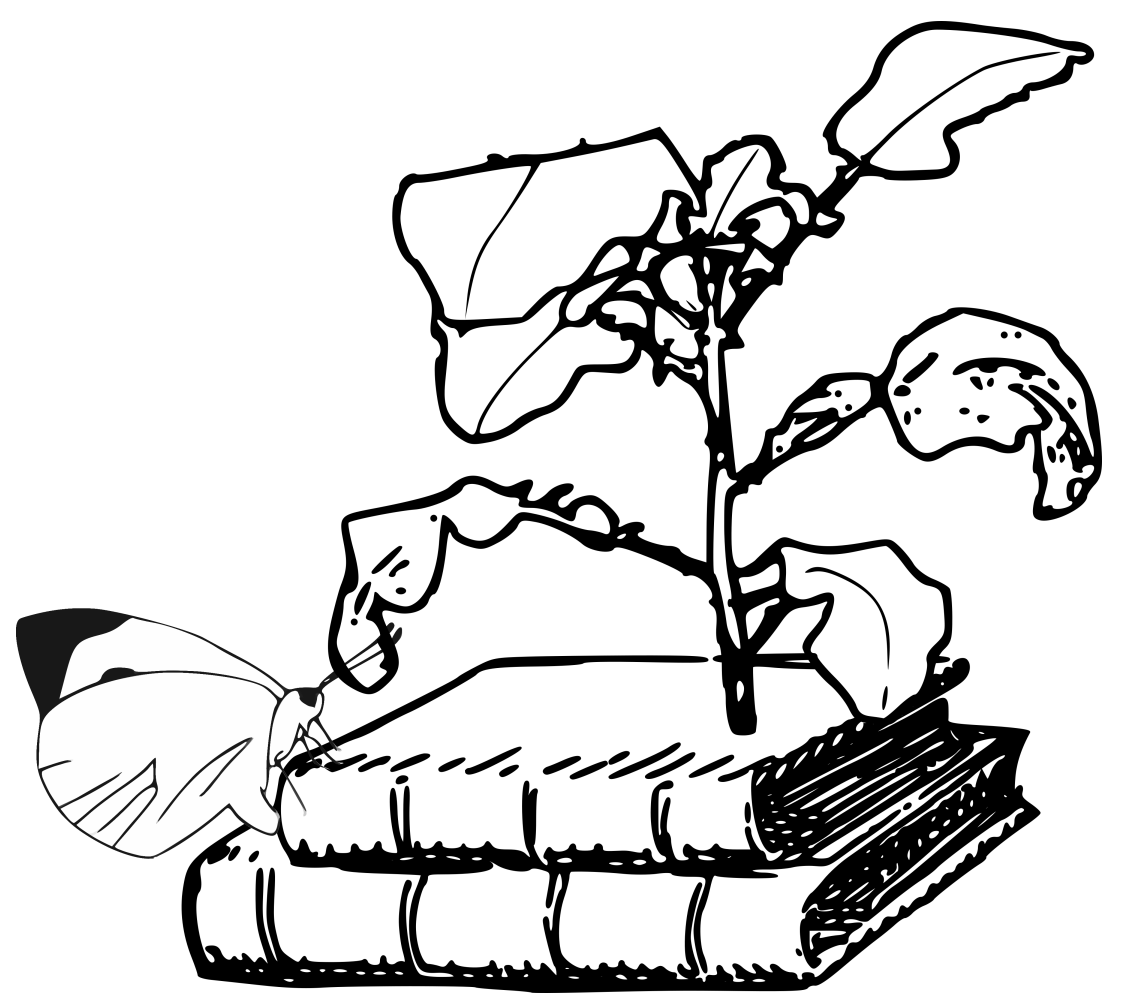




\section{Chapter 7 Bibliography}

Aartsma, Y., Bianchi, F. J. J. A., van der Werf, W., Poelman, E. H. and Dicke, M. (2017). "Herbivore-induced plant volatiles and tritrophic interactions across spatial scales". In: New Phytologist 216.4, pp. 1054-1063.

Aartsma, Y., Leroy, B., van der Werf, W., Dicke, M., Poelman, E. H. and Bianchi, F. J. J. A. (2019). "Intraspecific variation in herbivore-induced plant volatiles influences the spatial range of plant-parasitoid interactions”. In: Oikos 128.1, pp. 77-86.

Adler, F. and Karban, R. (1994). "Defended fortresses or moving targets - another model of inducible defenses inspired by military metaphors". In: American Naturalist 144.5, pp. 813-832.

Afentoulis, D. G., Cusumano, A., Greenberg, L., Caarls, L. and Fatouros, N. E. (2021). "Attraction of Trichogramma wasps to butterfly oviposition-induced plant volatiles depends on Brassica species, wasp strain and leaf necrosis". In: Frontiers in Ecology and Evolution 9.

Agrawal, A. A. (2001). "Phenotypic plasticity in the interactions and evolution of species”. In: Science 294.5541, pp. 321-326.

- (2002). "Herbivory and maternal effects: Mechanisms and consequences of transgenerational induced plant resistance”. In: Ecology 83.12, pp. 3408-3415.

Agrawal, A. A. and Karban, R. (2000). "Specificity of constitutive and induced resistance: Pigment glands influence mites and caterpillars on cotton plants". In: Entomologia Experimentalis et Applicata 96.1, pp. 39-49.

Agrawal, A. A. and van Zandt, P. A. (2003). "Ecological play in the coevolutionary theatre: genetic and environmental determinants of attack by a specialist weevil on milkweed". In: Journal of Ecology 91.6, pp. 1049-1059.

Agrawal, A. A. (2011). "Current trends in the evolutionary ecology of plant defence". In: Functional Ecology 25.2, pp. 420-432.

Albani, M. C., Castaings, L., Wötzel, S., Mateos, J. L., Wunder, J., Wang, R., Reymond, M., Coupland, G. and Yu, H. E. (2012). "PEP1 of Arabis alpina is encoded by two overlapping genes that contribute to natural genetic variation in perennial flowering". In: PLoS Genetics 8, e1003130.

Allen, P. E. (2010). "Group size effects on survivorship and adult development in the gregarious larvae of Euselasia chrysippe (Lepidoptera, Riodinidae)". In: Insectes Sociaux 57.2, pp. 199-204. 
Aluja, M., Díaz-Fleischer, F. and Arredondo, J. (2004). "Nonhost status of commercial Persea americana 'Hass' to Anastrepha ludens, Anastrepha obliqua, Anastrepha serpentina, and Anastrepha striata (Diptera: Tephritidae) in Mexico". In: Journal of Economic Entomology 97.2, pp. 293-309.

Arganda-Carreras, I., Kaynig, V., Rueden, C., Eliceiri, K. W., Schindelin, J., Cardona, A. and Seung, H. S. (2017). "Trainable Weka Segmentation: a machine learning tool for microscopy pixel classification". In: Bioinformatics 33.15, pp. 2424-2426.

Austel, N., Eilers, E. J., Meiners, T. and Hilker, M. (2016). "Elm leaves 'warned' by insect egg deposition reduce survival of hatching larvae by a shift in their quantitative leaf metabolite pattern". In: Plant, Cell \& Environment 39.2, pp. 366-376.

Balbyshev, N. F. and Lorenzen, J. H. (1997). "Hypersensitivity and egg drop: A novel mechanism of host plant resistance to Colorado potato beetle (Coleoptera: Chrysomelidae)". In: Journal of Economic Entomology 90.2, pp. 652-657.

Baldwin, I. T. (2010). "Plant volatiles”. In: Current Biology 20.9, R392-R397.

Balint-Kurti, P. (2019). "The plant hypersensitive response: concepts, control and consequences". In: Molecular Plant Pathology 20.8, pp. 1163-1178.

Bandoly, M., Grichnik, R., Hilker, M. and Steppuhn, A. (2016). "Priming of anti-herbivore defence in Nicotiana attenuata by insect oviposition: Herbivore-specific effects". In: Plant, Cell \& Environment 39.4, pp. 848-859.

Bandoly, M., Hilker, M. and Steppuhn, A. (2015). "Oviposition by Spodoptera exigua on Nicotiana attenuata primes induced plant defence against larval herbivory". In: Plant Journal 83.4, pp. 661-672.

Baruha, I. K., Panda, D., M. V., J., Das, D., Acharjee, S., Sen, P. and Sarmah, B. K. (2017). "Bruchid egg induced transcript dynamics in developing seeds of black gram (Vigna mungo)". In: PLoS ONE 12.4, e0176337.

Bassetti, N., Caarls, L., Verbaarschot, P., Bouwmeester, K., Zwaan, B., Bonnema, G., Schranz, M. E. and Fatouros, N. E. (2021). Genetic basis of butterfly egg-induced hypersenstive response hints to single dominat locus containingintracellular receptor cluster in Brassica nigra. In preparation.

Becerra, J. X. (2015). "Macroevolutionary and geographical intensification of chemical defense in plants driven by insect herbivore selection pressure". In: Current Opinion in Insect Science 8, pp. 15-21. 


\section{Chapter 7 Bibliography}

Beilstein, M. A., Al-Shehbaz, I. A. and Kellogg, E. A. (2006). "Brassicaceae phylogeny and trichome evolution”. In: American Journal of Botany 93.4, pp. 607-619.

Beilstein, M. A., Al-Shehbaz, I. A., Mathews, S. and Kellogg, E. A. (2008). "Brassicaceae phylogeny inferred from phytochrome A and ndhF sequence data: tribes and trichomes revisited". In: American Journal of Botany 95.10, pp. 1307-1327.

Benedict, J. H., Leigh, T. F. and Hyer, A. H. (1983). "Lygus hesperus (Heteroptera: Miridae) oviposition behavior, growth, and survival in relation to cotton trichome density". In: Environmental Entomology 12.2, pp. 331-335.

Beran, F., Köllner, T. G., Gershenzon, J. and Tholl, D. (2019). "Chemical convergence between plants and insects: biosynthetic origins and functions of common secondary metabolites". In: New Phytologist 223.1, pp. 52-67.

Berenbaum, M. (1983). "Coumarins and caterpillars: A case for coevolution”. In: Evolution 37.1, pp. 163-179.

Berenbaum, M. and Feeny, P. (1981). "Toxicity of angular furanocoumarins to swallowtail butterflies: Escalation in a coevolutionary arms race?" In: Science 212.4497, pp. 927-929.

Bertea, C. M., Casacci, L. P., Bonelli, S., Zampollo, A. and Barbero, F. (2020). "Chemical, physiological and molecular responses of host plants to lepidopteran egg-laying". In: Frontiers in Plant Science 10, 1768.

Beyaert, I. and Hilker, M. (2014). "Plant odour plumes as mediators of plant-insect interactions". In: Biological Reviews 89.1, pp. 68-81.

Beyaert, I., Koepke, D., Stiller, J., Hammerbacher, A., Yoneya, K., Schmidt, A., Gershenzon, J. and Hilker, M. (2012). 'Can insect egg deposition 'warn' a plant of future feeding damage by herbivorous larvae?" In: Proceedings of the Royal Society B-Biological Sciences 279.1726, pp. 101-108.

Birke, A. and Aluja, M. (2018). "Do mothers really know best? Complexities in testing the preference-performance hypothesis in polyphagous frugivorous fruit flies". In: Bulletin of Entomological Research 108.5, pp. 674-684.

Bittner, N., Trauer-Kizilelma, U. and Hilker, M. (2017). "Early plant defence against insect attack: involvement of reactive oxygen species in plant responses to insect egg deposition”. In: Planta 245.5, pp. 993-1007. 
Blum, M. S. and Hilker, M. (2002). "Chemical protection of insect eggs". In: Chemoecology of Insect Eggs and Egg Deposition. Ed. by M. Hilker and T. Meiners. Blackwell, Berlin and Oxford, pp. 61-90.

Bonnet, C., Lassueur, S., Ponzio, C., Gols, R., Dicke, M. and Reymond, P. (2017). “Combined biotic stresses trigger similar transcriptomic responses but contrasting resistance against a chewing herbivore in Brassica nigra". In: BMC Plant Biology 17.1, 127.

Bos, J. I. B., Prince, D., Pitino, M., Maffei, M. E., Win, J. and Hogenhout, S. A. (2010). "A functional genomics approach identifies candidate effectors from the aphid species Myzus persicae (green peach aphid)”. In: PLoS Genetics 6.11, e1001216.

Bouwmeester, H., Schuurink, R. C., Bleeker, P. M. and Schiestl, F. (2019). "The role of volatiles in plant communication”. In: The Plant Journal 100.5, pp. 892-907.

Braby, M. F., Vila, R. and Pierce, N. E. (2006). "Molecular phylogeny and systematics of the Pieridae (Lepidoptera: Papilionoidea): higher classification and biogeography". In: Zoological Journal of the Linnean Society 147.2, pp. 239-275.

Brattsten, L. B. (1991). "Bioengineering of crop plants and resistant biotype evolution in insects: Counteracting coevolution". In: Archives of Insect Biochemistry and Physiology 17.4, pp. 253-267.

Brock, A., Herzfeld, T., Paschke, R., Koch, M. and Dräger, B. (2006). "Brassicaceae contain nortropane alkaloids". In: Phytochemistry 67.18, pp. 2050-2057.

Bruce, T. J. A., Midega, C. A. O., Birkett, M. A., Pickett, J. A. and Khan, Z. R. (2010). "Is quality more important than quantity? Insect behavioural responses to changes in a volatile blend after stemborer oviposition on an African grass". In: Biology Letters 6.3, pp. 314-317.

Bruce, T. J. A., Wadhams, L. J. and Woodcock, C. M. (2005). "Insect host location: a volatile situation". In: Trends in Plant Science 10.6, pp. 269-274.

Bruessow, F., Gouhier-Darimont, C., Buchala, A., Metraux, J.-P. and Reymond, P. (2010). "Insect eggs suppress plant defence against chewing herbivores". In: Plant Journal 62.5 , pp. 876-885.

Bryant, S. R., Thomas, C. D. and Bale, J. S. (2000). "Thermal ecology of gregarious and solitary nettle-feeding nymphalid butterfly larvae”. In: Oecologia 122.1, pp. 1-10.

van der Burgh, A. M. and Joosten, M. H. A. J. (2019). "Plant immunity: Thinking outside and inside the box". In: Trends in Plant Science 24.7, pp. 587-601. 


\section{Chapter 7 Bibliography}

Bustos-Segura, C., Poelman, E. H., Reichelt, M., Gershenzon, J. and Gols, R. (2017). "Intraspecific chemical diversity among neighbouring plants correlates positively with plant size and herbivore load but negatively with herbivore damage". In: Ecology Letters 20.1, pp. 87-97.

Caarls, L., Bassetti, N., van Doesburg, F., Verbaarschot, P., van Loon, J. J. A., Schranz, M. E. and Fatouros, N. E. (2021). "Deciphering Brassica plant defence responses to cabbage white butterfly egg-associated molecular patterns". In: bioRxiv, 2021.03.29.437462. eprint: https://www.biorxiv.org/content/early/2021/03/29/2021.03.29.437462.full.pdf.

Chew, F. S. and Renwick, J. A. A. (1995). "Host plant choice in Pieris butterflies". In: Chemical Ecology of Insects 2. Ed. by R. T. Cardé and W. J. Bell. Boston, MA: Springer US, pp. 214-238.

Chung, S. H., Rosa, C., Scully, E. D., Peiffer, M., Tooker, J. F., Hoover, K., Luthe, D. S. and Felton, G. W. (2013). "Herbivore exploits orally secreted bacteria to suppress plant defenses". In: Proceedings of the National Academy of Sciences of the United States of America 110.39, pp. 15728-15733.

Cipollini, D., Walters, D. and Voelckel, C. (2017). "Costs of resistance in plants: From theory to evidence". In: Annual Plant Reviews online. American Cancer Society, pp. 263-307.

Clark, B. R. and Faeth, S. H. (1998). "The evolution of egg clustering in butterflies: A test of the egg desiccation hypothesis". In: Evolutionary Ecology 12.5, pp. 543-552.

Clausen, C. P. (1976). "Phoresy among entomophagous insects". In: Annual Review of Entomology 21.1, pp. 343-368.

Cohen, J. A. (1985). "Differences and similarities in cardenolide contents of queen and monarch butterflies in Florida and their ecological and evolutionary implications". In: Journal of Chemical Ecology 11.1, pp. 85-103.

Colazza, S., Fucarino, A., Peri, E., Salerno, G., Conti, E. and Bin, F. (2004). "Insect oviposition induces volatile emission in herbaceous plants that attracts egg parasitoids". In: Journal of Experimental Biology 207.1, pp. 47-53.

Consales, F., Schweizer, F., Erb, M., Gouhier-Darimont, C., Bodenhausen, N., Bruessow, F., Sobhy, I. and Reymond, P. (2012). "Insect oral secretions suppress wound-induced responses in Arabidopsis”. In: Journal of Experimental Botany 63.2, pp. 727-737.

Conti, E., Salerno, G., Leombruni, B., Frati, F. and Bin, F. (2010). "Short-range allelochemicals from a plant-herbivore association: a singular case of oviposition-induced 
synomone for an egg parasitoid”. In: Journal of Experimental Biology 213.22, pp. 39113919.

Cook, S. M., Khan, Z. R. and Pickett, J. A. (2007). "The use of push-pull strategies in integrated pest management”. In: Annual Review of Entomology 52.1, pp. 375-400.

Courtney, S. P. (1984). "The evolution of egg clustering by butterflies and other insects". In: The American Naturalist 123.2, pp. 276-281.

Craig, T. P. and Ohgushi, T. (2002). "Preference and performance are correlated in the spittlebug Aphrophora pectoralis on four species of willow". In: Ecological Entomology 27.5, pp. 529-540.

Cusumano, A., Weldegergis, B. T., Colazza, S., Dicke, M. and Fatouros, N. E. (2015). "Attraction of egg-killing parasitoids toward induced plant volatiles in a multi-herbivore context”. In: Oecologia 179.1, pp. 163-174.

Das, D., Baruha, I. K., Panda, D., Paswan, R. R., Acharjee, S. and Sarmah, B. K. (2021). "Bruchid beetle ovipositioning mediated defense responses in black gram pods". In: BCM Plant Biology 21, 38.

Daudi, A. and O'Brien, J. A. (2012). "Detection of hydrogen peroxide by dab staining in Arabidopsis leaves". In: Bio-protocol 2.18.

David, W. A. L. and Gardiner, B. O. C. (1962). "Oviposition and the hatching of the eggs of Pieris brassicae (L.) in a laboratory culture”. In: Bulletin of Entomological Research 53.01, pp. 91-109.

De Moraes, C. M., Mescher, M. C. and Tumlinson, J. H. (2001). "Caterpillar-induced nocturnal plant volatiles repel conspecific females”. In: Nature 410, pp. 577-580.

Degen, T., Dillmann, C., Marion-Poll, F. and Turlings, T. C. J. (2004). "High genetic variability of herbivore-induced volatile emission within a broad range of maize inbred lines". In: Plant Physiology 135.4, pp. 1928-1938.

Denno, R. F. and Benrey, B. (1997). "Aggregation facilitates larval growth in the neotropical nymphalid butterfly Chlosyne janais". In: Ecological Entomology 22.2, pp. 133141.

Des Marais, D. L., Hernandez, K. M. and Juenger, T. E. (2013). “Genotype-by-environment interaction and plasticity: Exploring genomic responses of plants to the abiotic environment". In: Annu Rev Ecol Evol S 44.1, pp. 5-29.

Després, L., David, J. P. and Gallet, C. (2007). "The evolutionary ecology of insect resistance to plant chemicals". In: Trends in ecology \& evolution 22.6, pp. 298-307. 


\section{Chapter 7 Bibliography}

Desurmont, G. A., Donoghue, M. J., Clement, W. L. and Agrawal, A. A. (2011). "Evolutionary history predicts plant defense against an invasive pest". In: Proceedings of the National Academy of Sciences of the United States of America 108.17, pp. 70707074.

Desurmont, G. A., Kerdellant, E. and Lambin, N. (2021). "Between a rock and an eggcrushing place: selection pressure from natural enemies and plant defences on eggs of the viburnum leaf beetle in its native range". In: Ecological Entomology 46.2, pp. 482486.

Desurmont, G. A. and Weston, P. A. (2011). "Aggregative oviposition of a phytophagous beetle overcomes egg-crushing plant defences". In: Ecological Entomology 36.3, pp. 335-343.

Desurmont, G. A., Weston, P. A. and Agrawal, A. A. (2014). "Reduction of oviposition time and enhanced larval feeding: two potential benefits of aggregative oviposition for the viburnum leaf beetle”. In: Ecological Entomology 39.1, pp. 125-132.

Dicke, M. and Sabelis, M. W. (1988). "How plants obtain predatory mites as bodyguards". In: Netherlands Journal of Zoology 38.2-4, pp. 148-165.

Dicke, M., Sabelis, M. W., Takabayashi, J., Bruin, J. and Posthumus, M. A. (1990). "Plant strategies of manipulating predator-prey interactions through allelochemicals: Prospects for application in pest control". In: Journal of Chemical Ecology 16.11, pp. 3091-3118.

Dicke, M. and Baldwin, I. T. (2010). "The evolutionary context for herbivore-induced plant volatiles: beyond the 'cry for help'”. In: Trends in Plant Science 15.3, pp. 167175.

Dicke, M. and Hilker, M. (2003). "Induced plant defences: from molecular biology to evolutionary ecology". In: Basic and Applied Ecology 4.1, pp. 3-14.

Doss, R. P., Oliver, J. E., Proebsting, W. M., Potter, S. W., Kuy, S. R., Clement, S. L., Williamson, R. T., Carney, J. R. and DeVilbiss, E. D. (2000). "Bruchins: Insect-derived plant regulators that stimulate neoplasm formation". In: Proceedings of the National Academy of Sciences of the United States of America 97.11, pp. 6218-6223.

Doss, R. P., Proebsting, W. M., Potter, S. W. and Clement, S. L. (1995). "Response of Np mutant of pea (Pisum sativum L) to pea weevil (Bruchus pisorum L) oviposition and extracts". In: Journal of Chemical Ecology 21.1, pp. 97-106. 
Du, Y., Poppy, G. M., Powell, W., Pickett, J. A., Wadhams, L. J. and Woodcock, C. M. (1998). "Identification of semiochemicals released during aphid feeding that attract parasitoid Aphidius ervi". In: Journal of Chemical Ecology 24.8, pp. 1355-1368.

Dudareva, N., Negre, F., Nagegowda, D. A. and Orlova, I. (2006). "Plant volatiles: Recent advances and future perspectives". In: Critical Reviews in Plant Sciences 25.5, pp. 417-440.

Edger, P. P., Heidel-Fischer, H. M., Bekaert, M., Rota, J., Glöckner, G., Platts, A. E., Heckel, D. G., Der, J. P., Wafula, E. K., Tang, M., Hofberger, J. A., Smithson, A., Hall, J. C., Blanchette, M., Bureau, T. E., Wright, S. I., dePamphilis, C. W., Schranz, M. E., Barker, M. S., Conant, G. C., Wahlberg, N., Vogel, H., Pires, J. C. and Wheat, C. W. (2015). "The butterfly plant arms-race escalated by gene and genome duplications". In: Proceedings of the National Academy of Sciences, 201503926.

Ehrlich, P. R. and Raven, P. H. (1964). "Butterflies and plants: A study in coevolution". In: Evolution 18.4, pp. 586-608.

Erb, M. (2018). "Volatiles as inducers and suppressors of plant defense and immunityorigins, specificity, perception and signaling”. In: Current Opinion in Plant Biology 44, pp. 117-121.

Erb, M. and Robert, C. A. M. (2016). "Sequestration of plant secondary metabolites by insect herbivores: Molecular mechanisms and ecological consequences". In: Current Opinion in Insect Science 14, pp. 8-11.

Farrell, B. D. and Mitter, C. (1994). "Adaptive radiation in insects and plants: Time and opportunity". In: American Zoologist 34.1, pp. 57-69.

Fatouros, N. E., Cusumano, A., Danchin, E. G. and Colazza, S. (2016). "Prospects of herbivore egg-killing plant defenses for sustainable crop protection". In: Ecology and Evolution, pp. 6906-6918.

Fatouros, N. E. and Huigens, M. E. (2012). "Phoresy in the field: natural occurrence of Trichogramma egg parasitoids on butterflies and moths". In: Biocontrol 57.4, pp. 493502.

Fatouros, N. E., Huigens, M. E., van Loon, J. J. A., Dicke, M. and Hilker, M. (2005). "Chemical communication: Butterfly anti-aphrodisiac lures parasitic wasps". In: Nature 433, pp. 704-704.

Fatouros, N. E., Lucas-Barbosa, D., Weldegergis, B. T., Pashalidou, F. G., van Loon, J. J. A., Dicke, M., Harvey, J. A., Gols, R. and Huigens, M. E. (2012). "Plant volatiles 


\section{Chapter 7 Bibliography}

induced by herbivore egg deposition affect insects of different trophic levels". In: PLoS One 7.8, e43607.

Fatouros, N. E., Paniagua Voirol, L., Drizou, F., Thi Doan, Q., Pineda, A., Frago, E. and van Loon, J. J. A. (2015). "Role of Large Cabbage White butterfly male-derived compounds in elicitation of direct and indirect egg-killing defenses in the black mustard". In: Frontiers in Plant Sciences 6, 794.

Fatouros, N. E., Pineda, A., Huigens, M. E., Broekgaarden, C., Shimwela, M. M., Figueroa Candia, I. A., Verbaarschot, P. and Bukovinszky, T. (2014). "Synergistic effects of direct and indirect defences on herbivore egg survival in a wild crucifer". In: Proceedings of the Royal Society B-Biological Sciences 281, 20141254.

Fei, M., Gols, R. and Harvey, J. A. (2014). "Seasonal phenology of interactions involving short-lived annual plants, a multivoltine herbivore and its endoparasitoid wasp". In: 83.1, pp. 234-244.

Feltwell, D. J. (1982). Large white butterfly: The biology, biochemistry and physiology of Pieris brassicae (Linnaeus). Springer.

Fernández-Bautista, N., Domínguez-Núñez, J. A., Moreno, M. M. C. and BerrocalLobo, M. (2016). "Plant tissue trypan blue staining during phytopathogen infection". In: Bio-protocol 6.24, e2078.

Firtzlaff, V., Oberlaender, J., Geiselhardt, S., Hilker, M. and Kunze, R. (2016). "Preexposure of Arabidopsis to the abiotic or biotic environmental stimuli "chilling" or "insect eggs" exhibits different transcriptomic responses to herbivory". In: Scientific Reports 6, 28544.

Flórez, L. V., Scherlach, K., Gaube, P., Ross, C., Sitte, E., Hermes, C., Rodrigues, A., Hertweck, C. and Kaltenpoth, M. (2017). “Antibiotic-producing symbionts dynamically transition between plant pathogenicity and insect-defensive mutualism". In: Nature Communications 8.1, 15172.

Fordyce, J. A. (2003). “Aggregative feeding of pipevine swallowtall larvae enhances hostplant suitability”. In: Oecologia 135.2, pp. 250-257.

Forister, M. L. (2004). "Oviposition preference and larval performance within a diverging lineage of lycaenid butterflies”. In: Ecological Entomology 29.3, pp. 264-272.

Forister, M. L., Nice, C. C., Fordyce, J. A. and Gompert, Z. (2009). "Host range evolution is not driven by the optimization of larval performance: the case of Lycaeides 
melissa (Lepidoptera: Lycaenidae) and the colonization of alfalfa". In: Oecologia 160.3, pp. 551-561.

Fortuna, T. M., Woelke, J. B., Hordijk, C. A., Jansen, J. J., van Dam, N. M., Vet, L. E. M. and Harvey, J. A. (2013). "A tritrophic approach to the preference-performance hypothesis involving an exotic and a native plant". In: Biological Invasions 15.11, pp. 23872401.

Fraenkel, G. S. (1959). "The raison d'être of secondary plant substances”. In: Science 129.3361, pp. 1466-1470.

Friberg, M., Posledovich, D. and Wiklund, C. (2015). "Decoupling of female host plant preference and offspring performance in relative specialist and generalist butterflies". In: Oecologia 178.4, pp. 1181-1192.

Futuyma, D. J. and Agrawal, A. A. (2009). "Macroevolution and the biological diversity of plants and herbivores". In: Proceedings of the National Academy of Sciences 106.43, pp. 18054-18061.

Garza, R., Vera, J., Cardona, C., Barcenas, N. and Singh, S. P. (2001). "Hypersensitive response of beans to Apion godmani (Coleoptera : Curculionidae)". In: Journal of Economic Entomology 94.4, pp. 958-962.

Gatehouse, J. A. (2002). "Plant resistance towards insect herbivores: a dynamic interaction”. In: New Phytologist 156.2, pp. 145-169.

Geiselhardt, S., Yoneya, K., Blenn, B., Drechsler, N., Gershenzon, J., Kunze, R. and Hilker, M. (2013). "Egg laying of Cabbage White Butterfly (Pieris brassicae) on Arabidopsis thaliana affects subsequent performance of the larvae". In: PLoS One 8.3, e59661.

Gershenzon, J. and Dudareva, N. (2007). "The function of terpene natural products in the natural world". In: Nature Chemical Biology 3.7, pp. 408-414.

Geuss, D., Stelzer, S., Lortzing, T. and Steppuhn, A. (2017). "Solanum dulcamara's response to eggs of an insect herbivore comprises ovicidal hydrogen peroxide production". In: Plant, Cell \& Environment 40.11, pp. 2663-2677.

Gols, R., Bukovinszky, T., van Dam, N. M., Dicke, M., Bullock, J. M. and Harvey, J. A. (2008a). "Performance of generalist and specialist herbivores and their endoparasitoids differs on cultivated and wild Brassica populations". In: Journal of Chemical Ecology 34.2, pp. 132-143. 


\section{Chapter 7 Bibliography}

Gols, R., Bullock, J. M., Dicke, M., Bukovinszky, T. and Harvey, J. A. (2011). “Smelling the wood from the trees: Non-linear parasitoid responses to volatile attractants produced by wild and cultivated cabbage". In: Journal of Chemical Ecology 37.8, 795.

Gols, R., van Dam, N. M., Raaijmakers, C. E., Dicke, M. and Harvey, J. A. (2009). “Are population differences in plant quality reflected in the preference and performance of two endoparasitoid wasps?” In: Oikos 118.5, pp. 733-743.

Gols, R., Wagenaar, R., Bukovinszky, T., van Dam, N. M., Dicke, M., Bullock, J. M. and Harvey, J. A. (2008b). "Genetic variation in defense chemistry in wild cabbages affects herbivores and their endoparasitoids”. In: Ecology 89.6, pp. 1616-1626.

Goltsev, Y., Rezende, G. L., Vranizan, K., Lanzaro, G., Valle, D. and Levine, M. (2009). "Developmental and evolutionary basis for drought tolerance of the Anopheles gambiae embryo". In: Developmental Biology 330.2, pp. 462-470.

Gouhier-Darimont, C., Schmiesing, A., Bonnet, C., Lassueur, S. and Reymond, P. (2013). "Signalling of Arabidopsis thaliana response to Pieris brassicae eggs shares similarities with PAMP-triggered immunity". In: Journal of Experimental Botany 64.2, pp. 665-674.

Gouhier-Darimont, C., Stahl, E., Glauser, G. and Reymond, P. (2019). "The Arabidopsis lectin receptor kinase LecRK-I.8 is involved in insect egg perception". In: Frontiers in Plant Science 10, 623.

Graser, G., Schneider, B., Oldham, N. J. and Gershenzon, J. (2000). "The methionine chain elongation pathway in the biosynthesis of glucosinolates in Eruca sativa (Brassicaceae)". In: Archives of Biochemistry and Biophysics 378.2, pp. 411-419.

Griese, E., Caarls, L., Bassetti, N., Mohammadin, S., Verbaarschot, P., Bukovinszkine'Kiss, G., Poelman, E. H., Gols, R., Schranz, M. E. and Fatouros, N. E. (2021). "Insect egg-killing: a new front on the evolutionary arms-race between brassicaceous plants and pierid butterflies". In: New Phytologist 230, pp. 341-353.

Griese, E., Dicke, M., Hilker, M. and Fatouros, N. E. (2017). "Plant response to butterfly eggs: inducibility, severity and success of egg-killing leaf necrosis depends on plant genotype and egg clustering”. In: Scientific Reports 7.1, 7316.

Griese, E., Pineda, A., Pashalidou, F. G., Iradi, E. P., Hilker, M., Dicke, M. and Fatouros, N. E. (2020). "Plant responses to butterfly oviposition partly explain preference-performance relationships on different brassicaceous species". In: Oecologia 192.2, pp. 463-475. 
Grimaldi, D. (1999). "The co-radiations of pollinating insects and angiosperms in the Cretaceous”. In: Annals of the Missouri Botanical Garden 86.2, pp. 373-406.

Gripenberg, S., Mayhew, P. J., Parnell, M. and Roslin, T. (2010). “A meta-analysis of preference-performance relationships in phytophagous insects". In: Ecology Letters 13.3, pp. 383-393.

Groux, R., Stahl, E., Gouhier-Darimont, C., Kerdaffrec, E., Jimenez-Sandoval, P., Santiago, J. and Reymond, P. (2020). "Arabidopsis natural variation in insect egg-induced cell death reveals a role for LECTIN RECEPTOR KINASE-I.1”. In: Plant Physiology 185.1, pp. 240-255.

Guo, X., Liu, J., Hao, G., Zhang, L., Mao, K., Wang, X., Zhang, D., Ma, T., Hu, Q., AlShehbaz, I. A. and Koch, M. A. (2017). "Plastome phylogeny and early diversification of Brassicaceae". In: BMC Genomics 18.1, 176.

Hall, J. C., Sytsma, K. J. and Iltis, H. H. (2002). "Phylogeny of Capparaceae and Brassicaceae based on chloroplast sequence data". In: American Journal of Botany 89.11, pp. 1826-1842.

Harris, M. O., Sandanayaka, M. and Griffin, A. (2001). "Oviposition preferences of the Hessian fly and their consequences for the survival and reproductive potential of offspring". In: Ecological Entomology 26.5, pp. 473-486.

Harvey, J. A., Witjes, L. M. A., Benkirane, M., Duyts, H. and Wagenaar, R. (2007). "Nutritional suitability and ecological relevance of Arabidopsis thaliana and Brassica oleracea as foodplants for the cabbage butterfly, Pieris rapae". In: Plant Ecology 189.1, pp. 117-126.

Heidel-Fischer, H. M. and Vogel, H. (2015). "Molecular mechanisms of insect adaptation to plant secondary compounds". In: Current Opinion in Insect Science 8, pp. 814.

Heidel-Fischer, H. M., Kirsch, R., Reichelt, M., Ahn, S.-J., Wielsch, N., Baxter, S. W., Heckel, D. G., Vogel, H. and Kroymann, J. (2019). "An insect counteradaptation against host plant defenses evolved through concerted neofunctionalization”. In: $M o$ lecular Biology and Evolution 36.5, pp. 930-941.

Heil, M. (2004). "Direct defense or ecological costs: Responses of herbivorous beetles to volatiles released by wild lima bean (Phaseolus lunatus)". In: Journal of Chemical Ecology 30.6, pp. 1289-1295. 


\section{Chapter 7 Bibliography}

Heil, M. and Karban, R. (2010). "Explaining evolution of plant communication by airborne signals". In: Trends in Ecology \& Evolution 25.3, pp. 137-144.

Heil, M. (2008). "Indirect defence via tritrophic interactions". In: New Phytologist 178.1, pp. 41-61.

Heil, M. and McKey, D. (2003). "Protective ant-plant interactions as model systems in ecological and evolutionary research". In: Annual Review of Ecology, Evolution, and Systematics 34.1, pp. 425-553.

Hilker, M. (1992). "Protective devices of early developmental stages in Pyrrhalta viburni (Coleoptera, Chrysomelidae)”. In: Oecologia 92.1, pp. 71-75.

Hilker, M. and Meiners, T. (2002). "Induction of plant responses to oviposition and feeding by herbivorous arthropods: a comparison". In: Entomologia Experimentalis et Applicata 104.1, pp. 181-192.

Hilker, M. and Fatouros, N. E. (2016). "Resisting the onset of herbivore attack: plants perceive and respond to insect eggs". In: Current Opinion in Plant Biology. Biotic interactions 32, pp. 9-16.

Hilker, M. and Fatouros, N. E. (2015). "Plant responses to insect egg deposition". In: Annual Review of Entomology 60, pp. 493-515.

Hilker, M. and Meiners, T. (2006). "Early herbivore alert: Insect eggs induce plant defense". In: Journal of Chemical Ecology 32.7, pp. 1379-1397.

- (2010). "How do plants "notice" attack by herbivorous arthropods?" In: Biological Reviews 85.2, pp. 267-280.

- (2011). "Plants and insect eggs: How do they affect each other?" In: Phytochemistry 72.13, pp. 1612-1623.

Hilker, M., Schwachtje, J., Baier, M., Balazadeh, S., Baeurle, I., Geiselhardt, S., Hincha, D. K., Kunze, R., Mueller-Roeber, B., Rillig, M. G., Rolff, J., Romeis, R., Schmuelling, T., Steppuhn, A., van Dongen, J., Whitcomb, S. J., Wurst, S., Zuther, E. and Kopka, J. (2016). "Priming and memory of stress responses in organisms lacking a nervous system”. In: Biological Reviews 91.4, pp. 1118-1133.

Hinton, H. E. (1981). Biology of insect eggs. Vol. I-III. Pergamon Press, Oxford.

Hopkins, R. J., van Dam, N. M. and van Loon, J. J. A. (2009). "Role of glucosinolates in insect-plant relationships and multitrophic interactions". In: Annual Review of Entomology 54, pp. 57-83. 
Huang, C.-H., Sun, R., Hu, Y., Zeng, L., Zhang, N., Cai, L., Zhang, Q., Koch, M. A., AlShehbaz, I., Edger, P. P., Pires, J. C., Tan, D.-Y., Zhong, Y. and Ma, H. (2015). "Resolution of Brassicaceae phylogeny using nuclear genes uncovers nested radiations and supports convergent morphological evolution”. In: Molecular Biology and Evolution 33.2, pp. 394-412.

Huigens, M. E. and Fatouros, N. E. (2013). "A hitch-hikers guide to parasitism: the chemical ecology of phoretic insect parasitoids". In: Chemical ecology of insect parasitoids. Ed. by E. Wajnberg and S. Colazza. John Wiley \& Sons, Ltd., pp. 86-111.

Jacobs, C. G. C., Rezende, G. L., Lamers, G. E. M. and van der Zee, M. (2013). “The extraembryonic serosa protects the insect egg against desiccation". In: Proceedings of the Royal Society B-Biological Sciences 280.1764, 20131082.

Jaenike, J. (1990). "Host specialization in phytophagous insects". In: Annual Review of Ecology and Systematics 21, pp. 243-273.

Jaretzky, R. and Wilcke, M. (1932). "Die herzwirksamen Glykoside von Cheiranthus cheiri und verwandten Arten”. In: Archiv der Pharmazie 270.2, pp. 81-94.

Jones, J. D. G. and Dangl, J. L. (2006). “The plant immune system”. In: Nature 444, pp. 323-329.

Kalske, A., Mutikainen, P., Muola, A., Scheepens, J. F., Laukkanen, L., Salminen, J.-P. and Leimu, R. (2014). "Simultaneous inbreeding modifies inbreeding depression in a plant-herbivore interaction”. In: Ecology Letters 17.2, pp. 229-238.

Kappers, I. F., Hoogerbrugge, H., Bouwmeester, H. J. and Dicke, M. (2011). "Variation in herbivory-induced volatiles among cucumber (Cucumis sativus L.) varieties has consequences for the attraction of carnivorous natural enemies". In: Journal of Chemical Ecology 37.2, pp. 150-160.

Karasov, T. L., Horton, M. W. and Bergelson, J. (2014a). "Genomic variability as a driver of plant-pathogen coevolution?" In: Current Opinion in Plant Biology 18, pp. 24 30.

Karasov, T. L., Kniskern, J. M., Gao, L., DeYoung, B. J., Ding, J., Dubiella, U., Lastra, R. O., Nallu, S., Roux, F., Innes, R. W., Barrett, L. G., Hudson, R. R. and Bergelson, J. (2014b). "The long-term maintenance of a resistance polymorphism through diffuse interactions". In: Nature 512, pp. 436-440.

Karban, R. (1983). "Induced responses of cherry trees to periodical cicada oviposition". In: Oecologia 59.2, pp. 226-231. 


\section{Chapter 7 Bibliography}

Kawahara, A. Y., Plotkin, D., Espeland, M., Meusemann, K., Toussaint, E. F. A., Donath, A., Gimnich, F., Frandsen, P. B., Zwick, A., dos Reis, M., Barber, J. R., Peters, R. S., Liu, S., Zhou, X., Mayer, C., Podsiadlowski, L., Storer, C., Yack, J. E., Misof, B. and Breinholt, J. W. (2019). "Phylogenomics reveals the evolutionary timing and pattern of butterflies and moths". In: Proceedings of the National Academy of Sciences 116.45, pp. 22657-22663.

Khan, Z. R., Ampong-Nyarko, K., Chiliswa, P., Hassanali, A., Kimani, S., Lwande, W., Overholt, W. A., Overholt, W. A., Picketta, J. A., Smart, L. E. and Woodcock, C. M. (1997). "Intercropping increases parasitism of pests". In: Nature 388, pp. 631-632.

Knolhoff, L. M. and Heckel, D. G. (2014). "Behavioral assays for studies of host plant choice and adaptation in herbivorous insects". In: Annual Review of Entomology 59.1, pp. 263-278.

König, M. A. E., Wiklund, C. and Ehrlén, J. (2016). "Butterfly oviposition preference is not related to larval performance on a polyploid herb". In: Ecology and Evolution 6.9, pp. 2781-2789.

Kowalewski, Z., Schindler, O., Jäger, H. and Reichstein, T. (1960). "Die Cardenolide von Erysimum perofskianum Fisch. et Mey. 2. Mitteilung. Glykoside und Aglykone, 217. Mitteilung". In: Helvetica Chimica Acta 43.5, pp. 1280-1290.

Kubitzki, K. and Gottlieb, O. R. (1984). "Micromolecular patterns and the evolution and major classification of angiosperms". In: TAXON 33.3, pp. 375-391.

Larsson, S. and Ekbom, B. (1995). "Oviposition mistakes in herbivorous insects: Confusion or a step towards a new host plant?” In: Oikos 72.1, pp. 155-160.

Lawrence, S. D., Novak, N. G. and Blackburn, M. B. (2007). "Inhibition of proteinase inhibitor transcripts by Leptinotarsa decemlineata Regurgitant in Solanum lycopersicum". In: Journal of Chemical Ecology 33.5, pp. 1041-1048.

Lawrence, S. D., Novak, N. G., Ju, C. J.-T. and Cooke, J. E. K. (2008). "Potato, Solanum tuberosum, defense against colorado potato beetle, Leptinotarsa decemlineata (Say): Microarray gene expression profiling of potato by colorado potato beetle regurgitant treatment of wounded leaves". In: Journal of Chemical Ecology 34.8, pp. 1013-1025.

Lewinington, Z. R. (2016). Dagvlinders voor Nederland en Vlaanderen. Kosmos Uitgevers Utrecht/Antwerpen. 
Little, D., Gouhier-Darimont, C., Bruessow, F. and Reymond, P. (2007). “Oviposition by pierid butterflies triggers defense responses in Arabidopsis". In: Plant Physiology 143.2, pp. 784-800.

Loreto, F., Dicke, M., Schnitzler, J.-P. and Turlings, T. C. J. (2014). "Plant volatiles and the environment". In: Plant, Cell \& Environment 37.8, pp. 1905-1908.

Lucas-Barbosa, D., van Loon, J. J. A., Gols, R., van Beek, T. A. and Dicke, M. (2013). "Reproductive escape: annual plant responds to butterfly eggs by accelerating seed production”. In: Functional Ecology 27.1, pp. 245-254.

Makarevich, I. F. (1992). "Cheiranthus allioni - A unique cardenolide-bearing plant". In: Chemistry of Natural Compounds 28.3, pp. 265-271.

Malcolm, S. B. and Brower, L. P. (1989). "Evolutionary and ecological implications of cardenolide sequestration in the monarch butterfly". In: Experientia 45.3, pp. 284295.

Mäntylä, E., Kleier, S., Kipper, S. and Hilker, M. (2017). "The attraction of insectivorous tit species to herbivore-damaged Scots pines". In: Journal of Ornithology 158.2, pp. 479-491.

Marchini, D., Bernini, L. F., Marri, L., Giordano, P. C. and Dallai, R. (1991). “The female reproductive accessory glands of the medfly Ceratitis capitata: Antibacterial activity of the secretion fluid". In: Insect Biochemistry 21.6, pp. 597-605.

Margaritis, L. (1985). "Structure and physiology of the eggshell". In: Comprehensive insect physiology, biochemistry and pharmacology. Ed. by G. Kerkut and L. Gilbert. 1st ed. Vol. 15. Pergamon Press, Oxford, pp. 153-230.

Martinez, G., Finozzi, M. V., Cantero, G., Soler, R., Dicke, M. and Gonzalez, A. (2017). "Oviposition preference but not adult feeding preference matches with offspring performance in the bronze bug Thaumastocoris peregrinus". In: Entomologia Experimentalis et Applicata 163.1, pp. 101-111.

Mayhew, P. J. (1997). "Adaptive patterns of host-plant selection by phytophagous insects". In: Oikos 79.3, pp. 417-428.

- (2001). "Herbivore host choice and optimal bad motherhood". In: Trends in Ecology \& Evolution 16.4, pp. 165-167.

Mazanec, Z. (1985). "Resistance of Eucalyptus marginata to Perthida glyphopa (Lepidoptera: Incurvariidae)”. In: Journal of the Australian Entomological Society 24, pp. 209221. 


\section{Chapter 7 Bibliography}

McCormick, A. C., Unsicker, S. B. and Gershenzon, J. (2012). "The specificity of herbivore-induced plant volatiles in attracting herbivore enemies". In: Trends in Plant Science 17.5, pp. 303-310.

Meiners, T., Hacker, N. K., Anderson, P. and Hilker, M. (2005). "Response of the elm leaf beetle to host plants induced by oviposition and feeding: the infestation rate matters". In: Entomologia Experimentalis et Applicata 115.1, pp. 171-177.

Meiners, T., Wäckers, F. and Lewis, W. J. (2003). "Associative learning of complex odours in parasitoid host location". In: Chemical Senses 28.3, pp. 231-236.

Miller, M. A., Pfeiffer, W. and Schwartz, T. (2010). "Creating the CIPRES Science Gateway for inference of large phylogenetic trees". In: pp. 1-8.

Mohammadin, S., Edger, P. P., Pires, J. C. and Schranz, M. E. (2015). "Positionallyconserved but sequence-diverged: identification of long non-coding RNAs in the Brassicaceae and Cleomaceae". In: BMC Plant Biology 15.1, 217.

Mohammadin, S., Wang, W., Liu, T., Moazzeni, H., Ertugrul, K., Uysal, T., Christodoulou, C. S., Edger, P. P., Pires, J. C., Wright, S. I. and Schranz, M. E. (2018). "Genome-wide nucleotide diversity and associations with geography, ploidy level and glucosinolate profiles in Aethionema arabicum (Brassicaceae)". In: Plant Systematics and Evolution 304.5, pp. 619-630.

Musser, R. O., Hum-Musser, S. M., Eichenseer, H., Peiffer, M., Ervin, G., Murphy, J. B. and Felton, G. W. (2002). "Herbivory: Caterpillar saliva beats plant defences - A new weapon emerges in the evolutionary arms race between plants and herbivores." In: Nature 416, pp. 599-600.

Myers, J. H. and Rothman, L. E. (1995). "Virulence and transmission of infectious diseases in humans and insects: evolutionary and demographic patterns". In: Trends in Ecology \& Evolution 10.5, pp. 194-198.

Nallu, S., Hill, J. A., Don, K., Sahagun, C., Zhang, W., Meslin, C., Snell-Rood, E., Clark, N. L., Morehouse, N. I., Bergelson, J., Wheat, C. W. and Kronforst, M. R. (2018). "The molecular genetic basis of herbivory between butterflies and their host plants". In: Nature Ecology \& Evolution 2.9, pp. 1418-1427.

Nikolov, L. A., Shushkov, P., Nevado, B., Gan, X., Al-Shehbaz, I. A., Filatov, D., Bailey, C. D. and Tsiantis, M. (2019). "Resolving the backbone of the Brassicaceae phylogeny for investigating trait diversity”. In: New Phytologist 222.3, pp. 1638-1651. 
Nufio, C. R. and Papaj, D. R. (2001). "Host marking behavior in phytophagous insects and parasitoids". In: Entomologia Experimentalis et Applicata 99.3, pp. 273-293.

Oates, C. N., Denby, K. J., Myburg, A. A., Slippers, B. and Naidoo, S. (2021). "Insect egg-induced physiological changes and transcriptional reprogramming leading to gall formation". In: Plant, Cell \& Environment 44.2, pp. 535-547.

Okamura, Y., Sato, A., Tsuzuki, N., Murakami, M., Heidel-Fischer, H. and Vogel, H. (2019). "Molecular signatures of selection associated with host plant differences in Pieris butterflies". In: Molecular Ecology 28.22, pp. 4958-4970.

Oñate-Sánchez, L. and Vicente-Carbajosa, J. (2008). "DNA-free RNA isolation protocols for Arabidopsis thaliana, including seeds and siliques". In: BMC Research Notes $1.1,93$.

Paniagua Voirol, L. R., Frago, E., Kaltenpoth, M., Hilker, M. and Fatouros, N. E. (2018). "Bacterial symbionts in Lepidoptera: Their diversity, transmission, and impact on the host”. In: Frontiers in Microbiology 9, 556.

Paniagua Voirol, L. R., Valsamakis, G., Lortzing, V., Weinhold, A., Johnston, P. R., Fatouros, N. E., Kunze, R. and Hilker, M. (2020). "Plant responses to insect eggs are not induced by egg-associated microbes, but by a secretion attached to the eggs". In: Plant, Cell \& Environment 43.8, pp. 1815-1826.

Pashalidou, F. G., Eyman, L., Sims, J., Buckley, J., Fatouros, N. E., De Moraes, C. M. and Mescher, M. C. (2020). "Plant volatiles induced by herbivore eggs prime defences and mediate shifts in the reproductive strategy of receiving plants". In: Ecology Letters 23.7, pp. 1097-1106.

Pashalidou, F. G., Fatouros, N. E., van Loon, J. J. A., Dicke, M. and Gols, R. (2015a). "Plant-mediated effects of butterfly egg deposition on subsequent caterpillar and pupal development, across different species of wild Brassicaceae". In: Ecological Entomology 40.4, pp. 444-450.

Pashalidou, F. G., Frago, E., Griese, E., Poelman, E. H., van Loon, J. J. A., Dicke, M. and Fatouros, N. E. (2015b). "Early herbivore alert matters: plant-mediated effects of egg deposition on higher trophic levels benefit plant fitness". In: Ecology Letters 18.9, pp. 927-936.

Pashalidou, F. G., Gols, R., Berkhout, B. W., Weldegergis, B. T., van Loon, J. J. A., Dicke, M. and Fatouros, N. E. (2015c). "To be in time: egg deposition enhances 


\section{Chapter 7 Bibliography}

plant-mediated detection of young caterpillars by parasitoids”. In: Oecologia 177.2, pp. 477-86.

Pashalidou, F. G., Lucas-Barbosa, D., van Loon, J. J. A., Dicke, M. and Fatouros, N. E. (2013). "Phenotypic plasticity of plant response to herbivore eggs: effects on resistance to caterpillars and plant development". In: Ecology 94.3, pp. 702-713.

Perumal, S., Koh, C. S., Jin, L., Buchwaldt, M., Higgins, E. E., Zheng, C., Sankoff, D., Robinson, S. J., Kagale, S., Navabi, Z.-K., Tang, L., Horner, K. N., He, Z., Bancroft, I., Chalhoub, B., Sharpe, A. G. and Parkin, I. A. P. (2020). “A high-contiguity Brassica nigra genome localizes active centromeres and defines the ancestral Brassica genome". In: Nature Plants 6.8, pp. 929-941.

Petzold-Maxwell, J., Wong, S., Arellano, C. and Gould, F. (2011). "Host plant direct defence against eggs of its specialist herbivore, Heliothis subflexa". In: Ecological Entomology 36.6, pp. 700-708.

Poelman, E. H., van Dam, N. M., van Loon, J. J. A., Vet, L. E. M. and Dicke, M. (2009a). "Chemical diversity in Brassica oleracea affects biodiversity of insect herbivores". In: Ecology 90.7, pp. 1863-1877.

Poelman, E. H., van Loon, J. J. A., Van Dam, N. M., Vet, L. E. M. and Dicke, M. (2010). "Herbivore-induced plant responses in Brassica oleracea prevail over effects of constitutive resistance and result in enhanced herbivore attack". In: Ecological Entomology 35.2, pp. 240-247.

Poelman, E. H., Oduor, A. M. O., Broekgaarden, C., Hordijk, C. A., Jansen, J. J., van Loon, J. J. A., van Dam, N. M., Vet, L. E. M. and Dicke, M. (2009b). "Field parasitism rates of caterpillars on Brassica oleracea plants are reliably predicted by differential attraction of Cotesia parasitoids". In: Functional Ecology 23.5, pp. 951-962.

Ponzio, C., Cascone, P., Cusumano, A., Weldegergis, B. T., Fatouros, N. E., Guerrieri, E., Dicke, M. and Gols, R. (2016). "Volatile-mediated foraging behaviour of three parasitoid species under conditions of dual insect herbivore attack". In: Animal Behaviour 111, pp. 197-206.

Price, P. W., Bouton, C. E., Gross, P., McPheron, B. A., Thompson, J. N. and Weis, A. E. (1980). "Interactions among 3 trophic levels - influence of plants on interactions between insect herbivores and natural enemies". In: Annual Review of Ecology and Systematics 11, pp. 41-65. 
Prokopy, R. J. and Roitberg, B. D. (2001). "Joining and avoidance behavior in nonsocial insects". In: Annual Review of Entomology 46, pp. 631-665.

R Core Team (2014). R: A Language and Environment for Statistical Computing.

- (2016). R: A Language and Environment for Statistical Computing. Vienna, Austria.

Ramos, R. R., Rodrigues, D. and Freitas, A. V. L. (2012). “Oviposition preference and larval performance in a Heliconius erato phyllis (Lepidoptera: Nymphalidae) population from southeastern Brazil: is there a positive relationship?" In: Journal of Natural History 46.11-12, pp. 669-681.

Rask, L., Andréasson, E., Ekbom, B., Eriksson, S., Pontoppidan, B. and Meijer, J. (2000). "Myrosinase: gene family evolution and herbivore defense in Brassicaceae". In: Plant Molecular Biology : An International Journal on Molecular Biology, Molecular Genetics and Biochemistry 42.1, pp. 93-114.

Rasmann, S., Köllner, T. G., Degenhardt, J., Hiltpold, I., Toepfer, S., Kuhlmann, U., Gershenzon, J. and Turlings, T. C. J. (2005). "Recruitment of entomopathogenic nematodes by insect-damaged maize roots". In: Nature 434, pp. 732-737.

Ratnasingham, S. and Herbert, P. D. N. (2007). "BOLD: The Barcode of Life Data System (http://www.barcodinglife.org)". In: Molecular Ecology Notes 7.3, pp. 355364.

Ratzka, A., Vogel, H., Kliebenstein, D. J., Mitchell-Olds, T. and Kroymann, J. (2002). "Disarming the mustard oil bomb". In: Proceedings of the National Academy of Sciences 99.17, pp. 11223-11228.

Réal, P., Robert, J.-C. and Morizot, C. (1967). "Eléments de biologie de Pieris ergane Geyer subsp. gallia Mezger dans les Pyrénées-Orientales”. In: Bulletin mensuel de la Société linnéenne de Lyon 36.6, pp. 257-268.

Reimann, A., Nurhayati, N., Backenköhler, A. and Ober, D. (2004). "Repeated evolution of the pyrrolizidine alkaloid-mediated defense system in separate angiosperm lineages". In: The Plant Cell 16.10, pp. 2772-2784.

Reymond, P. (2013). "Perception, signaling and molecular basis of oviposition-mediated plant responses". In: Planta 238.2, pp. 247-258.

Rezende, G. L., Martins, A. J., Gentile, C., Farnesi, L. C., Pelajo-Machado, M., Peixoto, A. A. and Valle, D. (2008). "Embryonic desiccation resistance in Aedes aegypti: presumptive role of the chitinized serosal cuticle”. In: BMC Developmental Biology 8,82 . 


\section{Chapter 7 Bibliography}

Roda, A., Halitschke, R., Steppuhn, A. and Baldwin, I. T. (2004). "Individual variability in herbivore-specific elicitors from the plant's perspective". In: Molecular Ecology 13.8, pp. 2421-2433.

Root, R. and Kareiva, P. (1984). "The search for resources by cabbage butterflies (Pieris rapae) - ecological consequences and adaptive significance of markovian movements". In: Ecology 65.1, pp. 147-165.

Rothschild, M. and Schoonhoven, L. M. (1977). "Assessment of egg load by Pieris brassicae (Lepidoptera-Pieridae)". In: Nature 266, pp. 352-355.

Rusman, Q., Poelman, E. H., Nowrin, F., Polder, G. and Lucas-Barbosa, D. (2019). "Floral plasticity: Herbivore-species-specific-induced changes in flower traits with contrasting effects on pollinator visitation". In: Plant, Cell \& Environment 42.6, pp. 1882 1896.

Sachdev-Gupta, K., Radke, C. D. and Renwick, J. A. (1993). “Antifeedant activity of cucurbitacins from Iberis amara against larvae of Pieris rapae". In: The International Journal of Plant Biochemistry 33.6, pp. 1385-1388.

Salerno, G., Frati, F., Marino, G., Ederli, L., Pasqualini, S., Loreto, F., Colazza, S. and Centritto, M. (2017). "Effects of water stress on emission of volatile organic compounds by Vicia faba, and consequences for attraction of the egg parasitoid Trissolcus basalis". In: Journal of Pest Science 90.2, pp. 635-647.

Scheirs, J., De Bruyn, L. and Verhagen, R. (2000). "Optimization of adult performance determines host choice in a grass miner". In: Proceedings of the Royal Society BBiological Sciences 267.1457, pp. 2065-2069.

Schillinger, J. A. and Gallun, R. L. (1968). "Leaf pubescence of wheat as a deterrent to the cereal leaf beetle, Oulema melanopus". In: Annals of the Entomological Society of America 61.4, pp. 900-903.

Schneider, C. A., Rasband, W. S. and Eliceiri, K. W. (2012). "NIH Image to ImageJ: 25 years of image analysis". In: Nature Methods 9.7, pp. 671-675.

Schoonhoven, L. M., Loon, J. J. A. V. and Dicke, M. (2005). Insect-plant biology. 2nd ed. Oxford University Press.

Schröder, R., Forstreuter, M. and Hilker, M. (2005). "A plant notices insect egg deposition and changes its rate of photosynthesis". In: Plant Physiology 138.1, pp. 470477. 
Seino, Y., Suzuki, Y. and Sogawa, K. (1996). “An ovicidal substance produced by rice plants in response to oviposition by the whitebacked planthopper, Sogatella furcifera (Horvath) (Homoptera: Delphacidae)". In: Applied Entomology and Zoology 31.4, pp. 467-473.

Shapiro, A. M. and DeVay, J. E. (1987). "Hypersensitivity reaction of Brassica nigra L. (Cruciferae) kills eggs of Pieris butterflies (Lepidoptera: Pieridae)". In: Oecologia 71.4, pp. 631-632.

Shapiro, A. M. (2002). "The Californian urban butterfly fauna is dependent on alien plants". In: Diversity and Distributions 8.1, pp. 31-40.

Al-Shehbaz, I. A. (2012). "A generic and tribal synopsis of the Brassicaceae (Cruciferae)". In: TAXON 61.5, pp. 931-954.

Silim Nahdy, M., Silim, S. N. and Ellis, R. H. (1999). "Some aspects of pod characteristics predisposing pigeonpea (Cajanus cajan (L.) Millsp.) to infestation by Callosobruchus chinensis (L.)" In: Journal of Stored Products Research 35.1, pp. 47-55.

Sillén-Tullberg, B. (1988). "Evolution of gregariousness in aposematic butterfly larvae: A phylogenetic analysis". In: Evolution 42.2, pp. 293-305.

Singer, M. S. and Stireman, J. O. (2005). "The tri-trophic niche concept and adaptive radiation of phytophagous insects". In: Ecology Letters 8.12, pp. 1247-1255.

Smallegange, R. C., van Loon, J. J. A., Blatt, S. E., Harvey, J. A., Agerbirk, N. and Dicke, M. (2007). "Flower vs. leaf feeding by Pieris brassicae: Glucosinolate-rich flower tissues are preferred and sustain higher growth rate". In: Journal of Chemical Ecology 33.10, pp. 1831-1844.

Stahl, E., Brillatz, T., Ferreira Queiroz, E., Marcourt, L., Schmiesing, A., Hilfiker, O., Riezman, I., Riezman, H., Wolfender, J.-L. and Reymond, P. (2020). "Phosphatidylcholines from Pieris brassicae eggs activate an immune response in Arabidopsis". In: eLife 9, e60293.

Stam, J. M., Kroes, A., Li, Y., Gols, R., van Loon, J. J. A., Poelman, E. H. and Dicke, M. (2014). "Plant interactions with multiple insect herbivores: From community to genes". In: Annual Review of Plant Biology 65, pp. 689-713.

Stamatakis, A. (2014). "RAxML version 8: a tool for phylogenetic analysis and postanalysis of large phylogenies”. In: Bioinformatics 30.9, pp. 1312-1313. 


\section{Chapter 7 Bibliography}

Stamp, N. (1980). "Egg deposition patterns in butterflies: why do some species cluster their eggs rather than deposit them singly?” In: American Naturalist 115.3, pp. 367380 .

Suzuki, R. and Shimodaira, H. (2006). "Pvclust: an R package for assessing the uncertainty in hierarchical clustering". In: Bioinformatics 22.12, pp. 1540-1542.

Swain, T. (1977). "Secondary compounds as protective agents". In: Annual Review of Plant Physiology 28.1, pp. 479-501.

Tamiru, A., Bruce, T. J. A., Midega, C. A. O., Woodcock, C. M., Birkett, M. A., Pickett, J. A. and Khan, Z. R. (2012). "Oviposition induced volatile emissions from African smallholder farmers' maize varieties". In: Journal of Chemical Ecology 38.3, pp. 231234.

Tamiru, A., Bruce, T. J. A., Richter, A., Woodcock, C. M., Midega, C. A. O., Degenhardt, J., Kelemu, S., Pickett, J. A. and Khan, Z. R. (2017). “A maize landrace that emits defense volatiles in response to herbivore eggs possesses a strongly inducible terpene synthase gene". In: Ecology and Evolution 7.8, pp. 2835-2845.

Tamiru, A., Bruce, T. J. A., Woodcock, C. M., Caulfield, J. C., Midega, C. A. O., Ogol, C. K. P. O., Mayon, P., Birkett, M. A., Pickett, J. A. and Khan, Z. R. (2011). "Maize landraces recruit egg and larval parasitoids in response to egg deposition by a herbivore". In: Ecology Letters 14.11, pp. 1075-1083.

Tamiru, A., Khan, Z. R. and Bruce, T. J. A. (2015). "New directions for improving crop resistance to insects by breeding for egg induced defence". In: Pests and resistance * Behavioural ecology 9, pp. 51-55.

Tamiru, A., Paliwal, R., Manthi, S. J., Odeny, D. A., Midega, C. A. O., Khan, Z. R., Pickett, J. A. and Bruce, T. J. A. (2020). "Genome wide association analysis of a stemborer egg induced "call-for-help" defence trait in maize". In: Scientific Reports 10.1, 11205.

Tasin, M., Bäckman, A.-C., Bengtsson, M., Ioriatti, C. and Witzgall, P. (2006). "Essential host plant cues in the grapevine moth". In: Naturwissenschaften 93.3, pp. 141144.

Thompson, J. N. (1988). "Evolutionary ecology of the relationship between oviposition preference and performance of offspring in phytophagous insects". In: Entomologia Experimentalis et Applicata 47.1, pp. 3-14. 
Thompson, J. (1988). "Variation in preference and specificity in monophagous and oligophagous swallowtail butterflies”. In: Evolution 42.1, pp. 118-128.

Thompson, J. and Pellmyr, O. (1991). "Evolution of oviposition behavior and host preference in Lepidoptera". In: Annual Review of Entomology 36, pp. 65-89.

Thorsteinson, A. J. (1960). "Host selection in phytophagous insects". In: Annual Review of Entomology 5.1, pp. 193-218.

Tolman, T. (2001). Photographic Guide To The Butterflies Of Britain And Europe. New York: Oxford University Press.

Tolman, T. and Lewington, R. (2009). Collins butterfly guide: The most complete guide to the butterflies of Britain and Europe. Illustrated edition. London: Harpercollins UK.

Turlings, T. C. and Erb, M. (2018). "Tritrophic interactions mediated by herbivoreinduced plant volatiles: mechanisms, ecological relevance, and application potential". In: Annual Review of Entomology 63.1, pp. 433-452.

Valsamakis, G., Bittner, N., Fatouros, N. E., Kunze, R., Hilker, M. and Lortzing, V. (2020). "Priming by timing: Arabidopsis thaliana adjusts its priming response to Lepidoptera eggs to the time of larval hatching”. In: Frontiers in Plant Science 11, 1969.

Wahlberg, N., Rota, J., Braby, M. F., Pierce, N. E. and Wheat, C. W. (2014). "Revised systematics and higher classification of pierid butterflies (Lepidoptera: Pieridae) based on molecular data”. In: Zoologica Scripta 43.6, pp. 641-650.

Weech, M.-H., Chapleau, M., Pan, L., Ide, C. and Bede, J. C. (2008). "Caterpillar saliva interferes with induced Arabidopsis thaliana defence responses via the systemic acquired resistance pathway". In: Journal of Experimental Botany 59.9, pp. 2437-2448.

Wetzel, W. C., Kharouba, H. M., Robinson, M., Holyoak, M. and Karban, R. (2016). "Variability in plant nutrients reduces insect herbivore performance". In: Nature 539.7629, pp. $425-427$.

Wheat, C. W., Vogel, H., Wittstock, U., Braby, M. F., Underwood, D. and MitchellOlds, T. (2007). "The genetic basis of a plant-insect coevolutionary key innovation". In: Proceedings of the National Academy of Sciences 104.51, pp. 20427-20431.

Wiemers, M., Chazot, N., Wheat, C. W., Schweiger, O. and Wahlberg, N. (2020). “A complete time-calibrated multi-gene phylogeny of the European butterflies". In: ZooKeys 938, pp. 97-124. 


\section{Chapter 7 Bibliography}

Wiklund, C. (1984). "Egg-laying patterns in butterflies in relation to their phenology and the visual apparency and abundance of their host plants". In: Oecologia 63.1, pp. 23-29.

Wiklund, C. and Åhrberg, C. (1978). "Host plants, nectar source plants, and habitat selection of males and females of Anthocharis cardamines (Lepidoptera)". In: Oikos 31.2, pp. 169-183.

Wiklund, C. and Friberg, M. (2008). "Enemy-free space and habitat-specific host specialization in a butterfly". In: Oecologia 157.2, pp. 287-294.

- (2009). "The evolutionary ecology of generalization: among-year variation in host plant use and offspring survival in a butterfly". In: Ecology 90.12, pp. 3406-3417.

Williams, K. S. and Gilbert, L. E. (1981). "Insects as selective agents on plant vegetative morphology - Egg mimicry reduces egg-laying by butterflies”. In: Science 212.4493, pp. $467-469$.

Winde, I. and Wittstock, U. (2011). "Insect herbivore counteradaptations to the plant glucosinolate-myrosinase system". In: Phytochemistry 72.13, pp. 1566-1575.

Windsor, A. J., Reichelt, M., Figuth, A., Svatoš, A., Kroymann, J., Kliebenstein, D. J., Gershenzon, J. and Mitchell-Olds, T. (2005). "Geographic and evolutionary diversification of glucosinolates among near relatives of Arabidopsis thaliana (Brassicaceae)". In: Phytochemistry 66.11, pp. 1321-1333.

Wittstock, U., Agerbirk, N., Stauber, E. J., Olsen, C. E., Hippler, M., Mitchell-Olds, T., Gershenzon, J. and Vogel, H. (2004). "Successful herbivore attack due to metabolic diversion of a plant chemical defense". In: Proceedings of the National Academy of Sciences of the United States of America 101.14, pp. 4859-4864.

Witzgall, P., Ansebo, L., Yang, Z., Angeli, G., Sauphanor, B. and Bengtsson, M. (2005). "Plant volatiles affect oviposition by codling moths". In: Chemoecology 15.2, pp. 7783.

Woods, H. A. (2010). "Water loss and gas exchange by eggs of Manduca sexta: Trading off costs and benefits". In: Journal of Insect Physiology. Insect Respiration 56.5, pp. 480-487.

Xia, J., Sinelnikov, I. V., Han, B. and Wishart, D. S. (2015). "MetaboAnalyst 3.0making metabolomics more meaningful”. In: Nucleic Acids Research 43.W1, W251W257. 
Xiu, C.-L., Pan, H.-S., Liu, B., Luo, Z.-X., Williams, L., Yang, Y.-Z. and Lu, Y.-H. (2019). "Perception of and behavioral responses to host plant volatiles for three $A d$ elphocoris species". In: Journal of Chemical Ecology 45.9, pp. 779-788.

Xue, J., Lenman, M., Falk, A. and Rask, L. (1992). “The glucosinolate-degrading enzyme myrosinase in Brassicaceae is encoded by a gene family". In: Plant Molecular Biology 18.2, pp. 387-398.

Yang, Y., Xu, J., Leng, Y., Xiong, G., Hu, J., Zhang, G., Huang, L., Wang, L., Guo, L., Li, J., Chen, F., Qian, Q. and Zeng, D. (2014). "Quantitative trait loci identification, fine mapping and gene expression profiling for ovicidal response to whitebacked planthopper (Sogatella furcifera Horvath) in rice (Oryza sativa L.)" In: BMC Plant Biology 14.1, 145.

Zangerl, A. R. (2003). "Evolution of induced plant responses to herbivores". In: Basic and Applied Ecology 4.1, pp. 91-103.

Züst, T., Strickler, S. R., Powell, A. F., Mabry, M. E., An, H., Mirzaei, M., York, T., Holland, C. K., Kumar, P., Erb, M., Petschenka, G., Gómez, J.-M., Perfectti, F., Müller, C., Pires, J. C., Mueller, L. A. and Jander, G. (2020). "Independent evolution of ancestral and novel defenses in a genus of toxic plants (Erysimum, Brassicaceae)". In: eLife 9, e51712. 



\section{Summary}

Since conquering the land some 400 million years ago, insects have been connected intricately with plants. While mutualistic relationships, such as pollination, between the two have been formed, many insects used and use plants as food sources. Thus, a coevolutionary arms race between plants and insect herbivores began. Plants developed sophisticated tools to protect themselves from being eaten. Direct defences such as mechanical barriers, toxins and low nutritional value are employed, as well as indirect defences, whereby natural enemies of herbivores are recruited to help plants fending off herbivores. However, especially specialist herbivores evolved many counteradaptations such as detoxifying or sequestering mechanisms, or the ability to choose less defended plants for oviposition and/or feeding. In this context, insect eggs could be considered a vulnerable stage as they are stuck in place, until larvae hatch from the eggs. The time between egg deposition and hatching of voracious larvae presents a special opportunity for plants to get rid of the future threat. Different plant species have been shown to use different egg-killing responses against various herbivores. Yet, so far plants and insect eggs have not been studied in an evolutionary perspective, as feeding insects and plants have largely been. Studying co-evolutionary interactions by considering egg killing and counteradaptations to it, could open new ways to understand and investigate insect-plant interactions.

Brassicaceae plants present a suitable study object to investigate egg-induced plant responses. Brassica nigra and Arabidopsis thaliana plants are well known to show various responses to Pieris eggs. Firstly, defence by formation of hypersensitive responselike (HR-like) necrosis at the oviposition site was observed for Brassica nigra some 35 years ago. Secondly, priming of anti-herbivore responses by Pieris egg deposition and subsequent effects on herbivores and higher trophic levels were recently reported. Thirdly, oviposition-induced plant volatiles (OIPVs) are induced which attract predators and parasitoids, and repel conspecific herbivores from oviposition. Lastly, variation of egg-killing responses between and within plant species has been recorded. These re- 


\section{Summary}

sponses are well documented for specific plant-insect egg pairs. However, often it is not clear how specific the responses elicited are. Are they elicited by insect eggs in general, or by specific species? Are they equally effective against different herbivore species? Additionally, it is as of yet, unknown if counteradaptations of insect species to the egginduced plant responses in B. nigra and other Brassicaceae exist. Finally, while HR-like necrosis is known as an egg-killing response in several, not closely related plant species, little is known about how widespread it is in the Brassicaceae.

In this thesis, I explore egg-induced responses of B. nigra and other brassicaceous plant species to Pieris brassicae and other pierid eggs. I focus on HR-like necrosis, OIPVs and how egg induction changes insect behaviour and caterpillar performance. Furthermore, I study how different plant species and plant accessions of the same species differ in their expression of HR-like necrosis and OIPVs.

The aim of this thesis is to shed light on the ecology and evolution of lepidopteran egginduced responses in Brassicaceae plants by comparing responses between and within plant species. I show how egg induction affects egg survival, caterpillar performance and the behaviour of conspecifics and their parasitoids, and how it may vary between different plant species and plant accessions. Additionally, I aim to provide evidence that HR-like necrosis in the Brassicaceae is specifically elicited by pierid butterfly eggs, and may be understood as a counteradaptation to these specialist herbivores.

Anti-herbivore responses and even anti-egg responses are well documented in the Brassicaceae and their specialist herbivores. In the plant family Brassicaceae both crop plants and model organisms such as A. thaliana are present. However, the first description of an egg-killing response in the Brassicaceae was in the wild crucifer $B$. nigra as a response to Pieris egg deposition. In chapter 2, I made use of the bitrophic B. nigra-P. brassicae system to study genotypic variation in induction of HR-like necrosis. I tested different plant accessions for their expression of HR-like necrosis and how it affected survival of clustered butterfly eggs. Both in greenhouse and field assays, some plant accessions expressed HR-like necrosis more frequently and more severely. Interestingly, even severe HR-like necrosis had no effect on egg survival. This was surprising since HR-like necrosis had previously been established as an egg-killing defence of $B$. nigra against a closely related solitary Pieris species, $P$. rapae.

I then tested whether or not the clustering of eggs provided protection against the eggkilling effect of HR-like necrosis. Indeed, egg mortality was higher when P. brassicae 
females were forced to lay single (non-touching) eggs instead of equally sized egg clutches in which eggs touched each other. Egg survival was lowered by HR-like necrosis for singly laid eggs. To explain this difference, I hypothesized that singly laid eggs are more likely to die from desiccation, as more surface area is exposed to the surrounding air. To test this hypothesis, I conducted an experiment were I showed that under low humidity conditions survival of single eggs was lower than of clustered eggs. Thus, I revealed not only that phenotypic variation in egg-induced plant responses of $B$. nigra exists but also demonstrated that egg-clustering could be understood as a potential counteradaptation to egg-killing plant responses.

Having proven variation in the expression of HR-like necrosis induced by clustered $P$. brassicae eggs within $B$. nigra accessions, I continued to research variation in another egg-induced trait, OIPVs and synergistic effects with HR-like necrosis. These volatiles are so-called indirect defences, known to attract egg parasitoids and repel conspecific herbivores. In chapter $\mathbf{3}$, I studied intraspecific variation between indirect egg-induced responses of $B$. nigra and consequences for $P$. brassicae and its parasitoids. In this tritrophic system, I collected and analysed plant volatiles from both control and $P$. brassicae egg-induced plants of two different plant accessions. Furthermore, I tested the behavioural response of gravid butterflies ( $P$. brassicae), egg parasitoids (Trichogramma evanescens) and larval parasitoids (Cotesia glomerata) to control and egg-induced plants of both accessions. The volatile blends of the differently treated accessions could not be separated by a cluster analysis, but I found that the emission of five individual compounds was induced or reduced by egg deposition. Additionally, two more volatiles were released in differing quantities by the different plant accessions. Both the preferences for OIPVs by egg-laying butterflies and host-foraging egg parasitoids were mainly influenced by egg induction rather than plant accessions. For $C$. glomerata, I showed that larval parasitism rates were different between control plants and plants previously exposed to eggs as well as between plant accessions. However, as larval parasitism occurred at least a week later than sampling of the volatile blends, these differences could not be linked to differences in volatile blends.

In the two previous chapters, my main focus was to study interactions between one butterfly species (P. brassicae) and one plant species (B. nigra). While this system is helpful for answering ecological questions, I increased the number of species to answer more evolutionary questions. Therefore, in chapter 4 , I additionally introduced a solit- 


\section{Summary}

ary butterfly species (P. rapae) and seven more brassicaceous plant species. I tested if the preference-performance hypothesis ( $\mathrm{PPH}$ ), according to which mothers will prefer to deposit eggs on plants where their offspring has the best performance, holds for the two butterfly species. In previous studies, egg induction has been neglected when testing the PPH. I correlated oviposition preferences with the survival of eggs, and the tested the performance of the feeding caterpillars by comparing control plants with plants previously induced by conspecific eggs. Only $P$. rapae showed a clear preference for some plant species over others, while $P$. brassicae did not show a preference. While HR-like necrosis lowered the survival of $P$. rapae eggs, females preferred to lay eggs on plant species more prone to express HR-like necrosis. After hatching and three days of feeding, caterpillar performance was not different between plant species, for neither butterfly species. However, $P$. brassicae caterpillars performed significantly worse on plants previously induced by eggs than on control plants, while $P$. rapae caterpillars showed no such difference. In contrast, if they fed on plants expressing HR-like necrosis to previously laid eggs, $P$. rapae caterpillars performed better than on plants that did not express HR. I did not find such a difference for P. brassicae caterpillars. After seven days of feeding, the performance of $P$. brassicae caterpillars correlated with oviposition preferences, but only on egg-induced plants. This difference between control and egg-induced plants was not observed for P. rapae caterpillars. In summary, I showed that egg-laying preference and offspring performance differed between the solitary and gregarious butterfly species. More importantly, egg induction and plant responses to eggs were necessary to fully understand preferences and performances of the two butterfly species.

After studying species-specific interactions between two Pieris species (adults, eggs and caterpillars) and plants (and their egg-induced responses) in previous chapters, I was interested in prevalence and specificity of the HR-like necrosis trait within the Brassicaceae. In chapter 5, I used a phylogenetic approach to unravel the potential co-adaptive relationship between pierid butterflies and Brassicaceae, in context of the evolved HR-like necrosis as an egg-killing trait. I made a wash of $P$. brassicae eggs to test 28 Brassicaceae and three Cleomaceae plant species for their ability to express HR-like necrosis upon induction. Only species from the tribe Brassiceae and a plant species of the Aethionemeae expressed HR-like necrosis. Conversely, I tested egg wash and eggs of eight pierid and one nymphalid butterflies, as well as the cabbage-feeding moths Plutella xylostella and Mamestra brassicae for their ability to induce HR-like 
necrosis in B. nigra plants. Eggs of Pieridae species adapted to feed on Brassicaceae plants (belonging to the subfamily Pierinae) induced necrosis, while Pieridae species feeding on host plants of other families, elicited a weak, chlorosis-like response. The nymphalid butterfly and the moths did not elicit any visible responses. The expression of the defence gene $P R 1$ mirrored this result. $P R 1$ expression was strongly induced by Pieris spp. and Anthocharis cardamines, both Brassicaceae specialists, while other Pieridae and the moths did not elicit $P R I$ expression or only a minor one. I also showed that HR-like necrosis lowered survival of single Pieris eggs in the field and the greenhouse, for four out of five plant species. In summary, my results suggest that eggs of Brassicaceae-specialist Pieridae induce HR-like necrosis which reduces egg survival. Thus, it seems some Brassicaceae evolved a trait fending off the initial step of attack by the butterflies, the deposited eggs. However, whether there is a single origin of eggkilling necrosis, with several losses, or at least two gains within the Brassicaceae cannot be concluded yet.

In the general discussion (chapter 6), I expand on the idea of viewing egg-induced plant responses in a co-evolutionary context. I show and discuss that direct egg-killing traits can be found in many different plant taxa, including gymnosperms, monocots and eudicots. While being widespread, only few examples in each taxon were investigated on egg-induced plant traits. HR-like necrosis is the most ubiquitous egg-induced response, and has been reported throughout the phylogenetic tree of plants. I discuss how both direct and indirect plant defences can lower egg survival, and compare it to the direct and indirect plant defences against feeding insects, for which coevolution has been demonstrated before. Furthermore, I point out several strategies employed by butterflies to circumvent egg killing by plant responses, especially for HR-like necrosis. Lastly, I speculate on the future direction of research on egg-induced plant responses. Unravelling the, still largely unknown, underlying mechanisms involved would not only provide fundamental knowledge about egg induction, but might also enable resistance breeding in crops. Identifying the, as of yet, unknown elicitor associated with Pieris eggs, will on the one hand be helpful in identifying receptor genes in the plants needed to perceive the deposited eggs. On the other hand, it could be used to identify the genes responsible for the biosynthesis of the elicitor in butterflies. With this knowledge it might be possible to explain why those butterflies cannot help but eliciting an egg-killing response.

Overall, my thesis has provided additional evidence that egg-killing traits vary between 


\section{Summary}

accessions of $B$. nigra. While some evidence on this existed before, my research was the first systematic approach to quantify the differences in HR-like necrosis expression. Furthermore, I was able to show for the first time, that clustering eggs effectively prevents egg killing despite HR-like necrosis expression. I showed that differences in single volatiles exist between $B$. nigra accessions, even if no direct link to insect behaviour was found. Additionally, I showed that to understand the evolution of oviposition choices by butterflies, egg induction needs to be taken into account as well. Only then the full picture of oviposition preference and caterpillar performance may be revealed. I showed that elicitation of HR-like necrosis in Brassicaceae is specific to species belonging to the Brassicaceae-feeding Pierinae. Finally, HR-like necrosis could only be induced in two Brassicaceae tribes (Brassiceae and Athionemeae), known as well accepted host plants of different Pieris butterflies. This provides some evidence for the counter-adaptive nature of insect egg-induced responses and insect eggs.

My thesis concludes that egg-induced responses are in a similar state of ongoing coadaptive arms race as anti-feeding responses. Furthermore, egg induction needs to be carefully considered when experimenting with plant-insect interactions. 


\section{Acknowledgements}

In the German language exists an idiom „Was lange währt, wird endlich gut“. It certainly „währte lang“ (took some time), and others may judge if it became good in the end. However, looking back I see that I could not have arrived here at the endpoint without the support of many people. At the beginning there is my family, parents as well as grandparents. Both generations did not attend university, but nevertheless they encouraged and supported my decision to study Biology greatly. Ohne die Ermunterung und Unterstützung meiner Eltern (Jens und Ilona) und Großeltern (Ingeborg und Horst) hätte ich es wohl kaum geschafft ein Biologiestudium anzufangen und dann auch noch nach Wageningen zu gehen, wo ich schließlich meine „Doktor-Familie“ traf.

Speaking of encouragement, there is also one Professor I need to thank in particular Johannes Steidle unterrichtete Biologie nicht nur so, dass Begeisterung für das Fach übersprang, er stellte letztlich auch den Kontakt nach Wageningen her, sodass ich ohne ihn wohl kaum diesen Weg eingeschlagen hätte. This change in my trajectory would have changed quite some things about my future way in life. Not going to Wageningen would mean, a different $\mathrm{PhD}$ thesis, if any.

In Wageningen I met many people along the way starting with my Master thesis. Already back then Nina and Marcel were my supervisors, with whom I planned my field experiment, and discussed the results. Nina especially went above and beyond, not only helping with the setting up of the field experiment, but later also made sure to give me opportunities and contacts to make my $\mathrm{PhD}$ project possible, as well as driving it forward when it was stuck. She continued being a great supervisor and got my other two supervisors, Monika Hilker and Eric involved. Both were greatly involved in changing and improving my writing, as well as aiding in discussing my results. Monika Hilker was also vital for starting up the PhD project, while Eric was important for finishing it. Different roles to be sure, but without them finalising the thesis could not be done.

Most the experiments conducted involved many different plants and insects, as well as organized timing and effort in planting. The Unifarm team with their expertise 
and handiwork were a great help for setting up and maintaining the field experiments throughout the years, as well as helping to rear the plants needed, for both greenhouse and field experiments. But they were of course not the only ones. Jeltje and Foteini and I all arranged field work around the same time and helped each other set up each others fields.

Making sure the butterflies were ready when needed, was only possible due to the efforts of the insect rearing team of the laboratory of Entomology, especially André, Leon, Joop and Frans. Additionally Dani and her students Kay and Lucille were organising the butterfly mating brilliantly. During my PhD I had the opportunity to work with Master and Bachelor students. Without their help some experiments would not have happened, or only happened in a different form. Especially Michele, Emilie and Ingeborg who contributed much to experiments in chapter 2. Bart on the other hand was working with me catching butterflies, and making use of them by letting them lay eggs on plants. For this tedious procedure, other people, colleagues and assistants were a great help as well. Lotte, Setareh, Niccoló, Gabriella, Patrick and Rieta were a great help here.

Of course no experiments would have been possible at all, if it were not for the plants used. For providing and recommending, as well as producing the B. nigra accessions in the first place, I thank Erik a lot. Berhane provided technical expertise for the collection and analysis of volatiles.

In general data analysis was helped a lot by the R-users team, to which I was introduced to by Jenny and Karen. While working on my thesis I lived together for a long time with Tila who took me in when my previous living arrangements came to an end. Shorter, but nevertheless impactful I lived together with other housemates mainly mentionable Qiona making the last few months living in Wagningen so much better. Technically, last but definitely not least of these housemates is Dr. Katarzyna, a scientist in her own right, whom I met in Wageningen, and later married in Germany. To her I like to say: Kasiu, chciałbym Ci podziękować za wszystko z całego serca. Jestem niesamowicie wdzięczny za to że mieliśmy okazje się poznać, że wytrwałaś przy mnie i zdecydowałaś dzielić swoje życie ze mną. Życzę nam obojgu by wszystkie lata które spędzimy ze sobą razem były tak dobre jak te pierwsze sześć. :)

I also had friends who stuck around, from studies or even A-levels in school, despite being already scattered all around Europe. Dr. rer. nat. Claas-Thido Pfaff, Dr. rer. 
nat. Sina Blessing (that is exactly how they wanted to be quoted :)) und Tobias haben mich aufgemuntert, wenn nötig, aufgenommen, wenn ich mal vor Ort war, und mich angestiftet Neues auszuprobieren. In Cologne I also met Kaori and Mihaly, who were also nice enough to ask me to translate for their wedding, which I gratefully accepted. In the end these friends were there when I needed them, and helped me greatly to finish my $\mathrm{PhD}$ journey. :) 



\section{About the author}

\section{Curriculum vitæ}

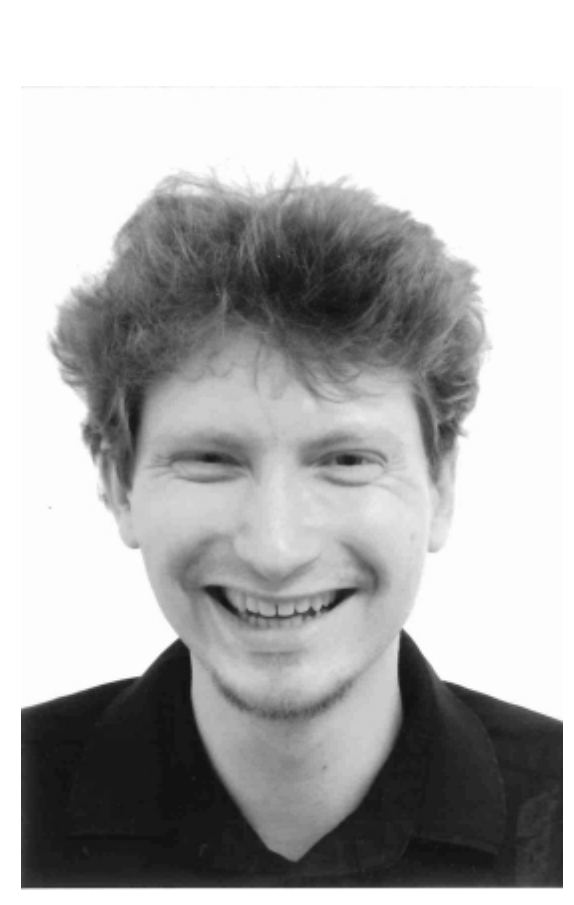

Eddie Griese was born on May 19th 1988 in Leipzig, Germany. He obtained first his Bachelor and later his Master degree in Biology from the University of Hohenheim (Stuttgart, Germany). While obtaining these degrees he also worked on the students council for Biology students. During his master degree studies he spent time at the University of Zurich working on the ecology and species distribution of the southern hemisphere grass family Restionaceae. He worked on his Master thesis at Wageningen University on the attraction of egg parasitoids to black mustard plants. Inspired by this field and laboratory work during his Master thesis he continued working on this topic after obtaining his Master degree, by starting his

$\mathrm{PhD}$. In the first year this was supported by a fellowship of the Integrated Research Training Group (IRTG) of the Collaborative Research Centre 973, Freie Universität Berlin, Germany, even though the research itself happened at the Laboratory of Entomology. After the first year and with help of the German Academic Exchange Service (DAAD) he continued the research at Wageningen University and Research both at the Laboratory of Entomology and later the Biosystematics Group at Wageningen University. 
About the author

\section{Publication list}

Foteini G. Pashalidou, Enric Frago, Eddie Griese, Erik H. Poelman, Joop J. A. van Loon, Marcel Dicke, Nina E. Fatouros (2015), Early herbivore alert matters: plant-mediated effects of egg deposition on higher trophic levels benefit plant fitness. Ecology Letters, 18: 927-936. https://doi.org/10.1111/ele.12470

Eddie Griese, Marcel Dicke, Monika Hilker, Nina E. Fatouros (2017) Plant response to butterfly eggs: inducibility, severity and success of egg-killing leaf necrosis depends on plant genotype and egg clustering. Scientific Reports, 7: 7316. https://doi.org/10. 1038/s41598-017-06704-Z

Eddie Griese, Ana Pineda, Foteini G. Pashalidou, Eleonora Pizzaro Iradi, Monika Hilker, Marcel Dicke, Nina E. Fatouros (2020) Plant responses to butterfly oviposition partly explain preference-performance relationships on different brassicaceous species. Oecologia, 192: 463-475. https://doi.org/10.1007/s00442-019-04590-y

Eddie Griese, Lotte Caarls, Niccolò Bassetti, Setareh Mohammadin, Patrick Verbaarschot, Gabriella Bukovinszkine' Kiss, Erik H. Poelman, Rieta Gols, M. Eric Schranz, Nina E. Fatouros (2021) Insect egg-killing: a new front on the evolutionary arms-race between brassicaceous plants and pierid butterflies. New Phytologist, 230: 341-353. https://doi.org/ s10.1111/nph.17145 


\section{Educational statement}

\section{Education Statement of the Graduate School}

Experimental Plant Sciences

$\begin{array}{ll}\text { Issued to: } & \text { Eddie Griese } \\ \text { Date: } & 04 \text { October } 2021 \\ \text { Group: } & \text { Biosystematics } \\ \text { University: } & \text { Wageningen University }\end{array}$

\section{1) Start-Up Phase}

- First presentation of your project

On the ecology and evolution of butterfly egg-killing defences in brassicaceous plants

Writing or rewriting a project proposal

DAAD proposal/DAAD follow up proposal

Writing a review or book chapter

- MSc courses

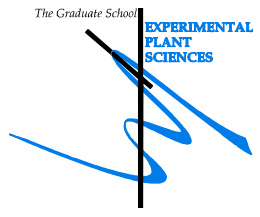

2) Scientific Exposure
EPS PhD student days
EPS PhD student days 'Get2Gether'
EPS PhD student days 'Get2Gether'
EPS theme symposia
EPS theme 1 symposium 'Developmental Biology of Plants'
EPS theme 3 symposium 'Metabolism and Adaptation'
Lunteren Days and other national platforms
Annual Meeting 'Experimental Plant Sciences' (Lunteren)
Annual Meeting of the Netherlands Entomological Society
Annual Meeting of the Netherlands Entomological Society
Annual Meeting of the Netherlands Entomological Society
Seminars (series), workshops and symposia
NESSC R workshop
Mini symposium on insect-microbe-plant interactions
12th EPS annual Workshop in Plant-Insect Interactions
WEES seminar: Endogenous viruses used by parasitic wasps to deliver virulence molecules to their hosts, Dr. Anne-Nathalie Volkoff
WEES seminar: Feeding on Electrons, Dr. Amelia-Elena Roaru
WEES seminar: Genetics and evolution of sex determination system in the common housefly, Dr. Daniel Bopp
WEES seminar: Macroecology and macroevolution of plant-pollinator interactions, Jeff Ollerton
WEES seminar: Spectrum dependent effects of light at night: The colour composition of illumination defines impact on behaviour and habitat
loss of different species, Dr. Kamiel Spoelstra
Seminar plus
International symposia and congresses
34th Annual Meeting of the International Society of Chemical Ecology (ISCE), Budapest, HU
10th European Plant Science Retreat (EPSR), Utrecht, NL
Presentations
Presentation: NESSC R workshop
Presentation: Mini symposium on insect-microbe-plant interactions
Presentation: EPSR
Presentation: Annual Meeting of the Netherlands Entomological Society
Poster: Annual Meeting of the Netherlands Entomological Society
Poster: ISCE Budapest
IAB interview
Excursions

\section{date}

22 Nov 2017

May 2014/Feb 2015

$\underline{\text { date }} \quad \underline{c p}$

15-16 Feb 2018

11-12 Feb 2019

30 Jan 2018

13 Mar 2018

09-10 Apr 2018

13 Dec 2013

15 Dec 2017

14 Dec 2018

01-02 Dec 2015

07 Apr 2017

09 Oct 2018

14 Dec 2017

25 Apr 2018

31 Oct 2018

31 May 2018

04 Jan 2019

12-18 Aug 2018

03-06 Jul 2018

01-02 Dec 2015

07 Apr 2017

04 Jul 2018

14 Dec 2018

15 Dec 2017

12-18 Aug 2018
1.5

7.5

$\underline{c p}$ 
About the author

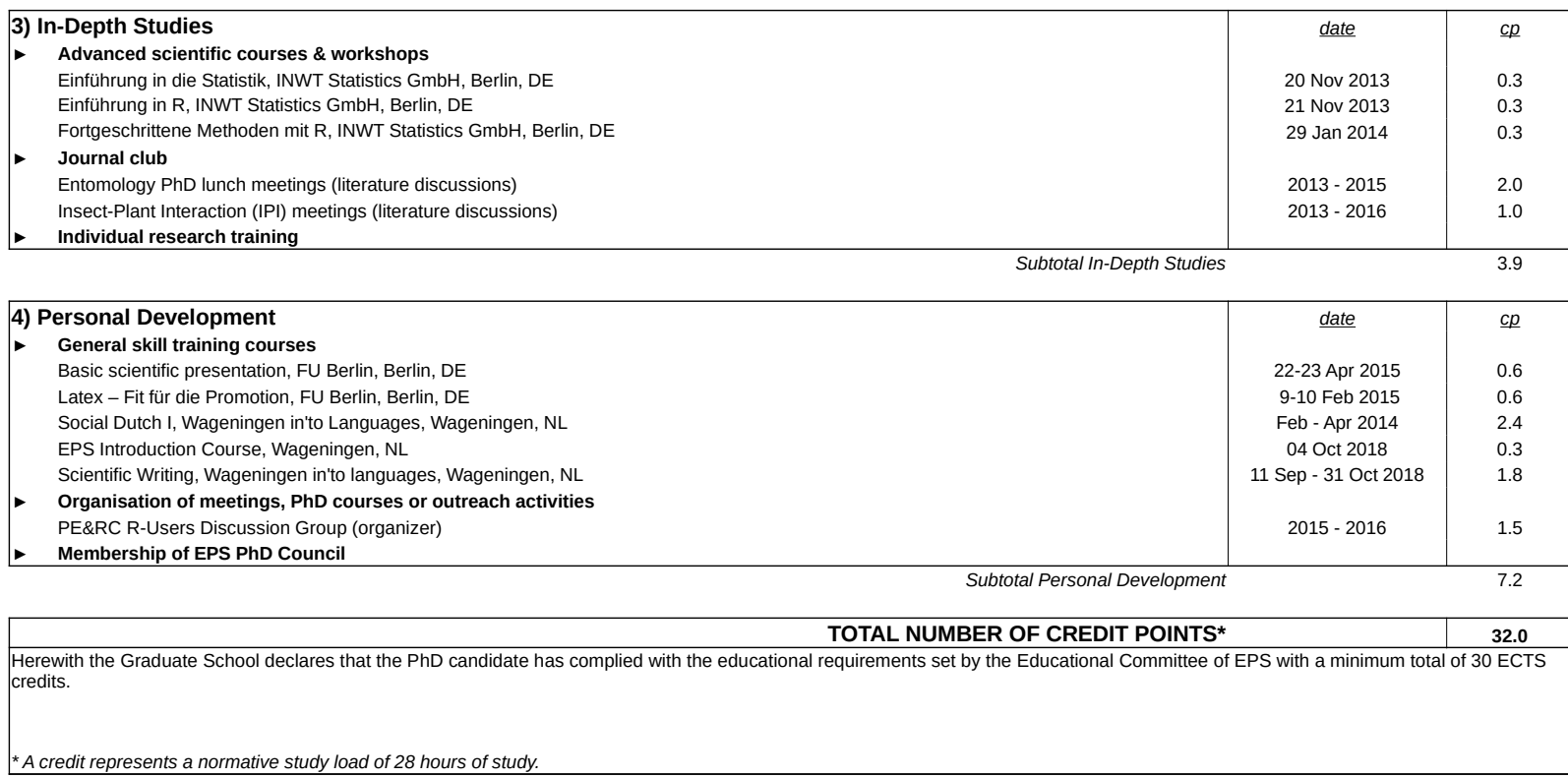


The research presented in this thesis was performed at the Laboratory of Entomology, and the Biosystematics Group, of Wageningen University \& Research (WUR) and was supported by the German Academic Exchange Service (DAAD) (Stipend no. 57044990), The Netherlands Organization for Scientific Research (NWO/TTW Vidi grant 14854 and connected Aspasia) and the German Research Foundation (CRC 973; www.sfb973 .de).

Cover illustrations by Eddie Griese

Cover design and thesis layout by Eddie Griese

Pictures on chapter title pages by Eddie Griese

Printed by GVO Printers \& Designers, The Netherlands 


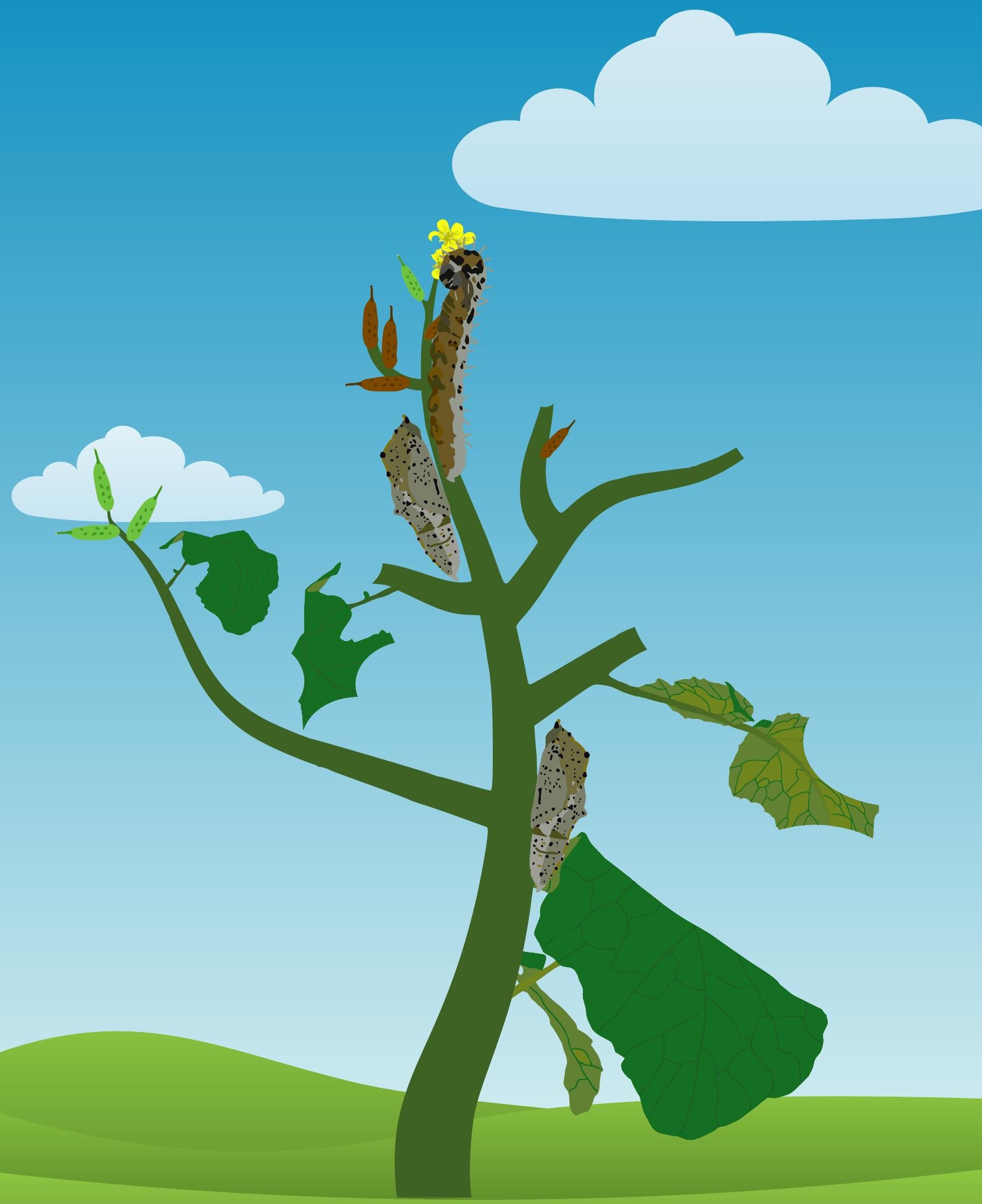

